\title{
HIGH PRESSURE GAS FILLED RF CAVITY BEAM TEST AT THE FERMILAB MUCOOL TEST AREA
}

\author{
BY \\ BEN FREEMIRE
}

Submitted in partial fulfillment of the requirements for the degree of Doctor of Philosophy in Physics in the Graduate College of the Illinois Institute of Technology

Approved

Advisor

Chicago, Illinois

May 2013 
(c) Copyright by

BEN FREEMIRE

May 2013 


\section{ACKNOWLEDGMENT}

I should first thank my advisers, Yağmur Torun and Dan Kaplan. Yağmur, thank you for giving me the leeway to get things done in my own fashion, a swift kick in the pants when I needed one, and for reminding me, "A graduate student's job is to graduate." I appreciate your helping me grow into a physicist. Dan, thank you for taking me on as a new graduate student and giving me the opportunity to work on MICE; it was a tremendous experience. My collaborators at the MTA, who are numerous, yet I shall name them: Alvin, Katsuya, Moses, Tom, Maria, Mukti, and Al. Alvin, your experience and insight has been invaluable. Thank you for always trying to make me think about the physics in things. Katsuya, your leadership throughout the course of my involvement with the HPRF program has made this work possible, thank you. Moses, my reference man. Thank you not only for helping me with the chemical side of things, but for your support as well. Thank you to my officemate of a number of years (and moves), Pierrick, who always provided me someone to talk to if I needed a break from the mundane.

And where would I be without my family? Mom and Dad, it was you who started me on this path many years ago, by setting down as a family to watch Star Trek. Your constant guidance and support have brought me to where I am today, thank you. Daniel and Kellie, I couldn't ask for better siblings, only that I could see you more often. I see myself in each of you, and you in me (which scares me sometimes!). To my parents-in-law, Sveta and Oleg. We may be continents apart, but you are always in my thoughts. Alina, I consider myself very lucky to have married someone with a sister whom I enjoy spending time with as much as I do with you. Malcolm, how to sum up all that you have done for me? You have been a colleague, a constant friend, and even let me share a bed with Claude. Thank you for going to a soccer game with me not too many years ago. And that brings me to 
Kseniya. I have never been one to believe in destiny, so I will just say that I'm glad you didn't have other plans on July 18, 2009. Your support and compassion have helped me more than you can know. 


\section{TABLE OF CONTENTS}

Page

ACKNOWLEDGEMENT . . . . . . . . . . . . . . . iii

LIST OF TABLES . . . . . . . . . . . . . . . . . viii

LIST OF FIGURES . . . . . . . . . . . . . . . . . . . . xiii

LIST OF SYMBOLS . . . . . . . . . . . . . . . . . xiv

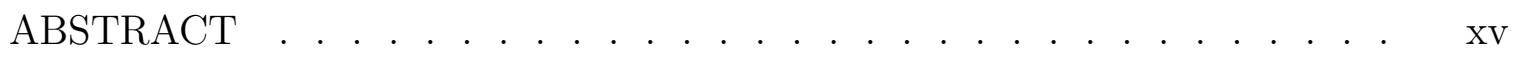

CHAPTER

1. INTRODUCTION . . . . . . . . . . . . . . . 1

1.1. Motivation . . . . . . . . . . . . . . . . . . 1

1.2. Theory . . . . . . . . . . . . . . . . 16

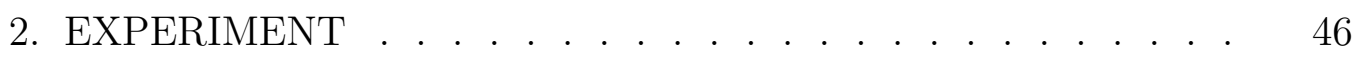

2.1. MuCool Test Area . . . . . . . . . . . . . . . . . . . 46

2.2. Beamline . . . . . . . . . . . . . . . . . . . . 46

2.3. Experimental Setup . . . . . . . . . . . . . . 50

2.4. Run Conditions . . . . . . . . . . . . . . . . . . . . 72

3. RESULTS . . . . . . . . . . . . . . . . 76

3.1. Energy Loss . . . . . . . . . . . . . . . . . . . . . . . 77

3.2. Recombination . . . . . . . . . . . . . 104

3.3. Attachment . . . . . . . . . . . . . . . . . . . 115

3.4. Ion-Ion Recombination . . . . . . . . . . . . . . . 138

4. CONCLUSION . . . . . . . . . . . . . 156

4.1. Neutrino Factory . . . . . . . . . . . . . . . . 156

4.2. Muon Collider . . . . . . . . . . . . . . . 157

4.3. Outlook . . . . . . . . . . . . . . . . . 160

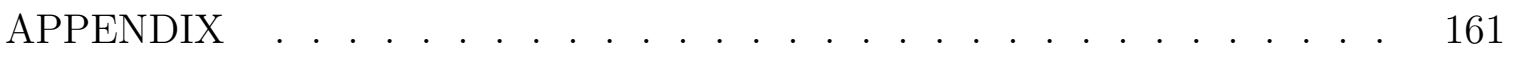

A. RUN CONDITIONS RUN I . . . . . . . . . . . . . 161

B. RUN CONDITIONS RUN II . . . . . . . . . . . . . . 166

C. CAVITY RESistanCE . . . . . . . . . . . . . . . . 179

D. ENERGY LOSS DATA . . . . . . . . . . . . . 187 
E. ELECTRON-ION RECOMBINATION DATA . . . . . . . . . 192

F. ATTACHMENT RATE DATA . . . . . . . . . . . . . . . 197

G. ION-ION RECOMBINATION DATA . . . . . . . . . . . . 200

BIBLIOGRAPHY . . . . . . . . . . . . . . . . . . . . . . . . . . 202 


\section{LIST OF TABLES}

Table

Page

1.1 RF requirements for a Neutrino Factory. . . . . . . . . . . . . . . 9

1.2 Proposed Muon Collider parameters . . . . . . . . . . . . . . . . 12

1.3 Proposed Neutrino Factory . . . . . . . . . . . . . . . . . . . 12

1.4 Ion mobilities in hydrogen. . . . . . . . . . . . . . . . . . . . . 28

1.5 Various three-body attachment coefficient measurements of electrons to oxygen in various gases at $300 \mathrm{~K}$. . . . . . . . . . . . . . . . 36

1.6 Ion-ion recombination rates. . . . . . . . . . . . . . . . . . . . 38

2.1 Fermilab $400 \mathrm{MeV}$ Linac specifications. . . . . . . . . . . . . . . 48

2.2 Toroid Inductance Measurements. . . . . . . . . . . . . . . . . . 57

2.3 HPRF cavity parameters. . . . . . . . . . . . . . . . . 61

2.4 Signal cable attenuation. . . . . . . . . . . . . . . . . . 66

2.5 Signal cable assignment. . . . . . . . . . . . . . . . . . . 67

2.6 Signal cable delay. . . . . . . . . . . . . . . . . . . . . . . . . 68

2.7 Gas Species and Pressures for Run II. . . . . . . . . . . . . . . . 75

3.1 Fits of $d w$ for pure hydrogen of the form $d w=c_{1} X^{c_{2}}$. . . . . . . 93

3.2 Fits of $d w$ for DA doped hydrogen of the form $d w=c_{1} X^{c_{2}}$. . . . . $\quad 99$

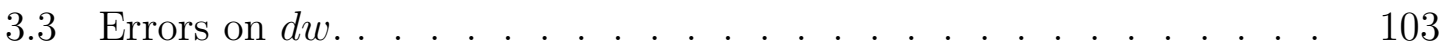

3.4 Fits of $\beta$ for pure hydrogen of the form $\beta=c_{1} X^{-c_{2}}$. . . . . . . 108

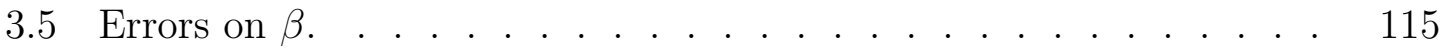

3.6 Fits of $\tau$ vs. concentration for DA doped hydrogen of the form $\tau=$ $c_{1} C^{-c_{2}}$. . . . . . . . . . . . . . . . . . . . . . . . 126

3.7 Fits of the form $\tau=c_{1} X^{c_{2}}$ for DA doped hydrogen. . . . . . . . . 131

3.8 Fits of the form $\tau=c_{1} P^{-c_{2}}$ for DA doped hydrogen. . . . . . . . . 137

3.9 Errors on $\tau$. . . . . . . . . . . . . . . . . . . . . . . . . . . . 138

3.10 Fits of $\eta$ vs. concentration for DA doped hydrogen of the form $\eta=$ $c_{1} C^{c_{2}}$. . . . . . . . . . . . . . . . . . . . . . . . . . . 
3.11 Fits of $\eta$ vs. $X$ for DA doped hydrogen of the form $\eta=c_{1} X^{c_{2}}$. . . 148

3.12 Fits of $\eta$ vs. $P$ for DA doped hydrogen of the form $\eta=c_{1} P^{c_{2}}$. . . 153

3.13 Errors on $\eta$. . . . . . . . . . . . . . . . . . . . . . . . . . . . 155

A.1 Run I run conditions. . . . . . . . . . . . . . . . . . . . . . . . 162

B.1 Run conditions for Run II. . . . . . . . . . . . . . . . . . . . . 167

C.1 Fitted time constants. . . . . . . . . . . . . . . . . . . . . 180

D.1 $d w$ for pure hydrogen. . . . . . . . . . . . . . . . . . . . . . . 188

D.2 $d w$ for dry air-doped hydrogen . . . . . . . . . . . . . . . . . . 189

E.1 $\beta$ for low beam intensity hydrogen, calculated at equilibrium . . . . 193

E.2 $\beta$ for high beam intensity hydrogen, calculated at equilibrium . . . 194

E.3 $\beta$ for hydrogen, calculated using the recursion formula. $\beta(E)=\beta_{0} E^{\beta e x p} 195$

F.1 $\tau$ for dry air-doped hydrogen, calculated using the recursion formula. 198

G.1 $\eta$ for dry air-doped hydrogen, calculated using the recursion formula. 201 


\section{LIST OF FIGURES}

Figure $\quad$ Page

1.1 Feynman diagram of a typical fusion process at a Muon Collider. . 2

1.2 Cross sections vs. center of mass energy for various processes at a lepton collider. . . . . . . . . . . . . . . . 3

1.3 Luminosity density comparison between a muon and electron collider at $3 \mathrm{TeV} \ldots \ldots \ldots \ldots$. . . . . . . . . . . . 4

1.4 Possible schematic for a Muon Collider. . . . . . . . . . . 5

1.5 A potential proton driver and target system for a Muon Collider based on an upgraded Project X. . . . . . . . . . . . . 6

1.6 A schematic of the MERIT experiment. . . . . . . . . . 6

1.7 Conceptual plot showing the phase rotation process for a Muon Collider. . . . . . . . . . . . . . . . . 7

1.8 The results of a simulation of particle energy and time spread before and after the phase rotation. . . . . . . . . . . 7

1.9 Cooling simulation for a possible Muon Collider cooling-channel. . 8

1.10 Schematic diagram of a Neutrino Factory. . . . . . . . . . . 11

1.11 Diagram of transverse ionization cooling. . . . . . . . . . . 13

1.12 Three examples of emittance exchange. . . . . . . . . . 15

1.13 Three possible $6 \mathrm{D}$ cooling-channels. . . . . . . . . . . . 15

1.14 Simulation of the magnetic focusing effect in an RF cavity. . . . . 18

1.15 Maximum accelerating field in a pillbox cavity vs. external magnetic field. . . . . . . . . . . . . . . . . . . 18

1.16 The first Townsend coefficient normalized to pressure vs. $X . \ldots 19$

1.17 Maximum gradient vs. pressure for different electrode materials. . 20

1.18 Maximum gradient vs. pressure for different gas species. . . . . . 21

1.19 Mean energy loss rate versus particle momentum in various materials. 24

1.20 An illustration of beam loading. . . . . . . . . . . . . 25

1.21 Electron drift velocity in hydrogen gas vs. $E / P \ldots \ldots 27$ 
1.22 Drift velocities for $\mathrm{H}_{3}^{+}$and $\mathrm{H}_{5}^{+}$in hydrogen. Reduced zero field mobility of $\mathrm{H}_{3}^{+}$in hydrogen. . . . . . . . . . . 27

1.23 Electron drift velocity and kinetic energy in hydrogen vs. E/N. . 28

1.24 Recombination rate vs. electron temperature for $\mathrm{H}_{3}^{+}$and $\mathrm{H}_{5}^{+} \ldots \ldots 30$

1.25 Effective recombination rate vs. hydrogen gas density at various gas temperatures. . . . . . . . . . . . . . . 31

1.26 Potential energy curves for $\mathrm{O}_{2}$ and $\mathrm{O}_{2}^{-} \ldots \ldots \ldots$

1.27 Attachment rate vs. hydrogen density. . . . . . . . . . . . 34

1.28 Attachment coefficients for oxygen and helium vs. electron energy. 34

1.29 Attachment coefficients and attachment rates of nitrogen vs. electron energy. . . . . . . . . . . . . . . . . . . . . . . . . 35

1.30 Attachment coefficient and attachment rate vs. oxygen concentration. 36

1.31 Ion-ion recombination rate. . . . . . . . . . . . . . 38

2.1 Linac hall engineering drawing. . . . . . . . . . . . . . . . . 49

2.2 MTA hall engineering drawing. . . . . . . . . . . . . 50

2.3 The experimental setup. . . . . . . . . . . . . . . . . 51

2.4 Scintillating screen. . . . . . . . . . . . . . . . . . . 52

2.5 Housing for the upstream and downstream toroids. . . . . . . 56

2.6 Downstream toroid calibration data. . . . . . . . . . . . 57

2.7 Linac toroid data from April 18, 2012 . . . . . . . . . . 58

2.8 Engineering drawing of the HPRF cavity. . . . . . . . . . 59

2.9 HPRF Cavity. . . . . . . . . . . . . . . . . . . . . . . . 60

2.10 Electric field vs. $z$ inside the HPRF cavity. . . . . . . . . . . 62

2.11 Circulator schematic. . . . . . . . . . . . . . . 63

2.12 The circulator installed in the MTA hall. . . . . . . . . . . 64

2.13 The hybrid installed in the MTA hall. . . . . . . . . . . 64

2.14 Cable response function vs. time. . . . . . . . . . . 65

2.15 Schematic for the cable timing calibration. . . . . . . . 66 
2.16 Schematic of a directional coupler. . . . . . . . . . . . . . . 69

2.17 Directional coupler closest to the HPRF cavity. . . . . . . . . . . 70

2.18 Layout of the gas shed. . . . . . . . . . . . . . . . . . . . . . 71

2.19 Schematic of the gas handling system. . . . . . . . . . . . . . . 71

3.1 A typical RF envelope. . . . . . . . . . . . . . . . . . . . 78

3.2 The equivalent circuit of a plasma and beam loaded cavity. . . . . 78

3.3 Typical cavity gradient decay fit. . . . . . . . . . . . . . . . 79

3.4 Typical beam intensity plot. . . . . . . . . . . . . . . . . . 81

3.5 Example of the electric field used for a $d w$ calculation. . . . . . . 82

3.6 Example of the power used for a $d w$ calculation. . . . . . . . . . 83

3.7 Example of the integrated number of protons used for a $d w$ calculation. 83

3.8 Beam intensities for all hydrogen data. . . . . . . . . . . . . . . 85

$3.996 \%$ peak voltage times. . . . . . . . . . . . . . . . . . 85

3.10 Example $d w$ vs. beam intensity fit at 300 psi. . . . . . . . . . . . 86

3.11 Example $d w$ vs. $X$ fit at 300 psi. . . . . . . . . . . . . . . . . . 87

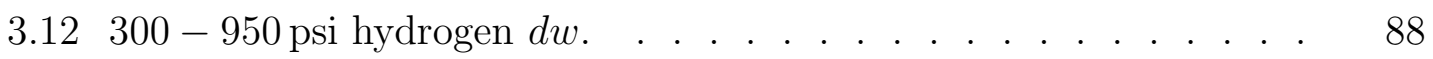

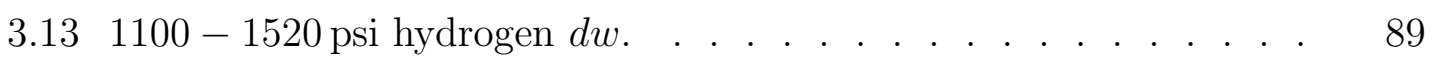

$3.14 d w$ for all pressures of hydrogen. . . . . . . . . . . . . . . . . . 90

3.15 Electron mobility in hydrogen vs. density. . . . . . . . . . . . . 91

3.16 The reciprocal of the normalized drift velocity of electrons in hydrogen vs. pressure. . . . . . . . . . . . . . . . . . . . . 92

3.17 Correction to $d E / d x$ with dry air dopant. . . . . . . . . . . . . 94

3.18 Correction to ionization energy with dry air dopant. . . . . . . . 94

$3.19 d w$ for 300 psi hydrogen doped with dry air. . . . . . . . . . 95

$3.20 d w$ for 735 psi hydrogen doped with dry air. . . . . . . . . . 95

$3.21 d w$ for 800 psi hydrogen doped with dry air. . . . . . . . . . . 96

$3.22 d w$ for 1100 psi hydrogen doped with dry air. . . . . . . . . . 96 
$3.23 d w$ for 1385 psi hydrogen doped with dry air. . . . . . . . . . . . 97

$3.24 d w$ for 1450 psi hydrogen doped with dry air. . . . . . . . . . . . 97

$3.25 d w$ for 1470 psi hydrogen doped with dry air. . . . . . . . . . . . 98

$3.26 d w$ comparison between electric and magnetic probes. . . . . . . 101

3.27 Energy loss for pure hydrogen including systematic errors. . . . . 102

3.28 Simulation of the beam in the HPRF cavity. . . . . . . . . . . . 105

3.29 Recombination rates at equilibrium for pure hydrogen. . . . . . . 107

3.30 Results of recursion formula. . . . . . . . . . . . . . . . . 110

3.31 Recombination rates. . . . . . . . . . . . . . . . . . . 112

3.32 Prediction of $\tau$. . . . . . . . . . . . . . . . . . . . . . . . . . 116

3.33 Fitted power curve for 300 psi hydrogen doped with dry air. . . . 118

3.34 Fitted power curve for 1470 psi hydrogen doped with dry air. . . . 119

3.35 Results of the recursion formula for dry air-doped hydrogen. . . . 120

3.36 Attachment time vs. DA concentration and $\mathrm{O}_{2}$ density. . . . . . . 122

3.37 Attachment time vs. DA concentration and $\mathrm{O}_{2}$ density. . . . . . 123

3.38 Attachment time vs. $X$ and average electron kintetic energy. . . . 128

3.39 Attachment time vs. $X$ and average electron kintetic energy. . . . 129

3.40 Attachment time vs. total pressure and number density. . . . . . 133

3.41 Attachment time vs. total pressure and number density. . . . . . 134

3.42 Attachment time vs. total pressure and number density. . . . . . 135

3.43 Electron kinetic energy data range. . . . . . . . . . . . . . . . 136

3.44 Ion-ion recombination rate vs. DA concentration and $\mathrm{O}_{2}$ density. . 140

3.45 Ion-ion recombination rate vs. DA concentration and $\mathrm{O}_{2}$ density. . 141

3.46 Ion-Ion recombination rate vs. $X$. . . . . . . . . . . . . . . 145

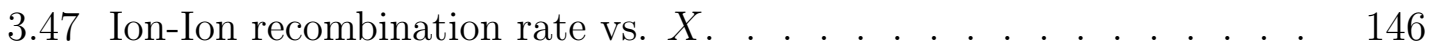

3.48 Ion-ion recombination rate vs. total pressure and number density. . 149

3.49 Ion-ion recombination rate vs. total pressure and number density. . 150 
3.50 Ion-ion recombination rate vs. total pressure and number density. .

4.1 Number of electron-hydrogen ion pairs and $\mathrm{O}_{2}^{-}$for one $\mathrm{RF}$ cycle with an initial using HCC parameters. . . . . . . . . . . . . . . 158

$4.2 d w$ per particle pair over one RF cycle based on HCC parameters. $\quad 159$

4.3 Energy loss over one RF cycle based on HCC parameters. . . . . 159 


\section{LIST OF SYMBOLS}

\begin{tabular}{|c|c|}
\hline Symbol & Definition \\
\hline$E$ & Electric field \\
\hline$P$ & Pressure \\
\hline$X$ & Electric field / pressure \\
\hline$T$ & Temperature \\
\hline$\epsilon$ & Kinetic energy \\
\hline$\beta$ & Electron-Ion recombination rate \\
\hline$\eta$ & Ion-Ion recombination rate \\
\hline$\tau$ & Attachment time \\
\hline$W_{i}$ & Average energy required to ionize a hydrogen molecule \\
\hline$h$ & Length of the accelerating gap of the HPRF cavity \\
\hline$R$ & Shunt impedance \\
\hline$Q$ & Quality factor \\
\hline$C$ & Capacitance \\
\hline$f$ & Frequency \\
\hline$\mu$ & Mobility \\
\hline$k_{B}$ & Boltzmann's constant \\
\hline
\end{tabular}




\begin{abstract}
The high energy physics community is continually looking to push the limits with respect to the energy and luminosity of particle accelerators. In the realm of leptons, only electron colliders have been built to date. Compared to hadrons, electrons lose a large amount of energy when accelerated in a ring through synchrotron radiation. A solution to this problem is to build long, straight accelerators for electrons, which has been done with great success. With a new generation of lepton colliders being conceived, building longer, more powerful accelerators is not the most enticing option. Muons have been proposed as an alternative particle to electrons. Muons lose less energy to synchrotron radiation and a Muon Collider can provide luminosity within a much smaller energy range than a comparable electron collider. This allows a circular collider to be built with higher attainable energy than any present electron collider. As part of the accelerator, but separate from the collider, it would also be possible to allow the muons to decay to study neutrinos. The possibility of a high energy, high luminosity muon collider and an abundant, precise source of neutrinos is an attractive one.
\end{abstract}

The technological challenges of building a muon accelerator are many and diverse. Because the muon is an unstable particle, a muon beam must be cooled and accelerated to the desired energy within a short amount of time. This requirement places strict requisites on the type of acceleration and focusing that can be used. Muons are generated as tertiary beams with a huge phase space, so strong magnetic fields are required to capture and focus them. Radio frequency (RF) cavities are needed to capture, bunch and accelerate the muons. Unfortunately, traditional vacuum RF cavities have been shown to break down in the magnetic fields necessary for capture and focusing.

To successfully operate RF cavities in strong magnetic fields, the idea of filling 
them with a high pressure gas in order to mitigate breakdown was proposed. The gas has the added benefit of providing cooling for the beam. Experiments were successfully performed using different gas species in a test cell cavity placed in a multi-Tesla magnetic field. These encouraging results lead to the necessity of a test closer to actual accelerator conditions, namely sending a beam of particles through the cavity. The electron-ion plasma created in the cavity by the beam absorbs energy and can degrade the accelerating electric field of the cavity. Electrons can recombine with hydrogen ions, however this process is slow compared to the bunch length and spacing. As electrons account for the majority of the energy loss in the cavity, their removal in a short time is highly desirable. The addition of an electronegative dopant gas can greatly decrease the lifetime of an electron in the cavity.

The results of two beam tests will be presented. The experimental variables cover a wide range of gas pressure, beam intensity, and cavity electric field. Measurements in pure hydrogen of the power consumption of electrons in the cavity indicate a range of energy loss between $10^{-18}$ and $10^{-16}$ joules per RF cycle per electron. When hydrogen doped with dry air is used, measurements of the power consumption indicate an energy loss range of $10^{-20}$ to $10^{-18}$ joules per RF cycle per ion, two orders of magnitude improvement over non-doped measurements. The rate at which electrons recombine with positively charged hydrogen ions ranges from $10^{-7}$ to $10^{-5} \frac{\mathrm{cm}^{3}}{\mathrm{~S}}$. The lifetime of electrons in a mixture of hydrogen gas and dry air has been measured from $<1 \mathrm{~ns}$, up to $200 \mathrm{~ns}$. Finally, the ion-ion recombination rate falls between $10^{-8}$ and $10^{-7} \frac{\mathrm{cm}^{3}}{\mathrm{~s}}$.

The results extrapolated to the parameters of a Neutrino Factory and Muon Collider indicate that a high pressure gas filled RF cavity will work in a coolingchannel for either machine. A demonstration experiment is warranted to prove this technology's validity. 


\section{CHAPTER 1 \\ INTRODUCTION}

\subsection{Motivation}

With the completion of the Large Hadron Collider (LHC) at the European Organization for Nuclear Research (CERN) and recent discovery of a Higgs-like boson $[2,3]$, building a particle accelerator that could study Higgs couplings and new physics related to the Higgs not achievable by the LHC is an attractive option. Additionally, the neutrino mixing angle $\theta_{13}$ has been measured to be non-zero [4-8], which provides the possibility of measuring $\mathrm{CP}$ violation in the lepton sector. A facility that could do this as well as determine the neutrino mass hierarchy and provide a more precise measurement of neutrino mixing parameters would be highly beneficial.

A muon accelerator could probe both of these areas of physics. A high luminosity Muon Collider could be designed with a center of mass energy at the Higgs resonance, or provide a multi-TeV machine with which to search for new physics, while a Neutrino Factory could be incorporated into such a complex before the collider to provide an abundant, well-characterized source of neutrinos.

1.1.1 Muon Collider. There are three main particle production channels of interest for a Muon Collider. The first is pair production, with the ratio, $R$, of the cross section for producing a particle, $X$, and its antiparticle to that of the cross section for electron-positron production being the figure of merit [1]:

$$
R \equiv \frac{\sigma\left(\mu^{+} \mu^{-} \rightarrow X+\bar{X}\right)}{\sigma_{Q E D}\left(\mu^{+} \mu^{-} \rightarrow e^{+} e^{-}\right)}
$$

For example, at $3 \mathrm{TeV}$, the rate for top quark production is $R=1.86$ [1].

The second channel is s-channel resonance. The cross section for production 
is $[1]$ :

$$
\sigma_{\mu^{+} \mu^{-} \rightarrow X}=\frac{\pi}{4 k^{2}}(2 J+1) \frac{\Gamma^{2}}{(E-M)^{2}+\Gamma^{2} / 4} B_{\mu^{+} \mu^{-}} B_{\text {visible }}
$$

where $k$ is the momentum of the incoming muon, $J$ is the spin of particle $X, E$ is the initial state energy, $M$ is the mass of particle $X, \Gamma B_{\mu^{+} \mu^{-}}$is the partial width of $X \rightarrow \mu^{+} \mu^{-}$and $\Gamma B_{\text {visible }}$ is the visible decay width of $X$. At peak resonance [1]:

$$
R_{\text {peak }}=3(2 J+1) \frac{B_{\mu^{+} \mu^{-}} B_{v i s i b l e}}{\alpha_{E M}^{2}}
$$

where $\alpha_{E M}^{2}$ is the electromagnetic coupling constant.

The final channel is fusion processes of the kind shown in Fig. 1.1. Here X is the particle of interest. Figure 1.2 shows the cross section for such a process vs. center of mass energy. Note the large cross section for the process $\mu^{+} \mu^{-} \rightarrow \bar{\nu}_{\mu} \nu_{\mu} H$.

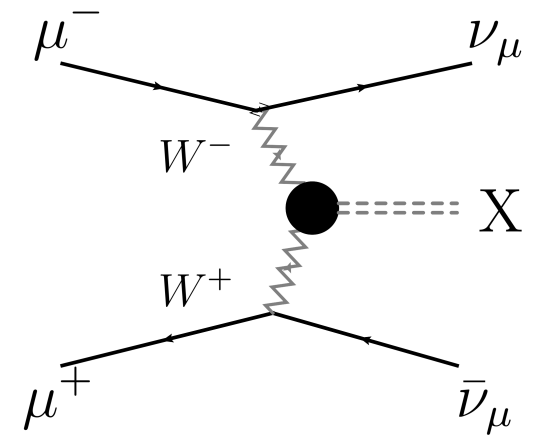

Figure 1.1. Feynman diagram of a typical fusion process at a Muon Collider [1]. 


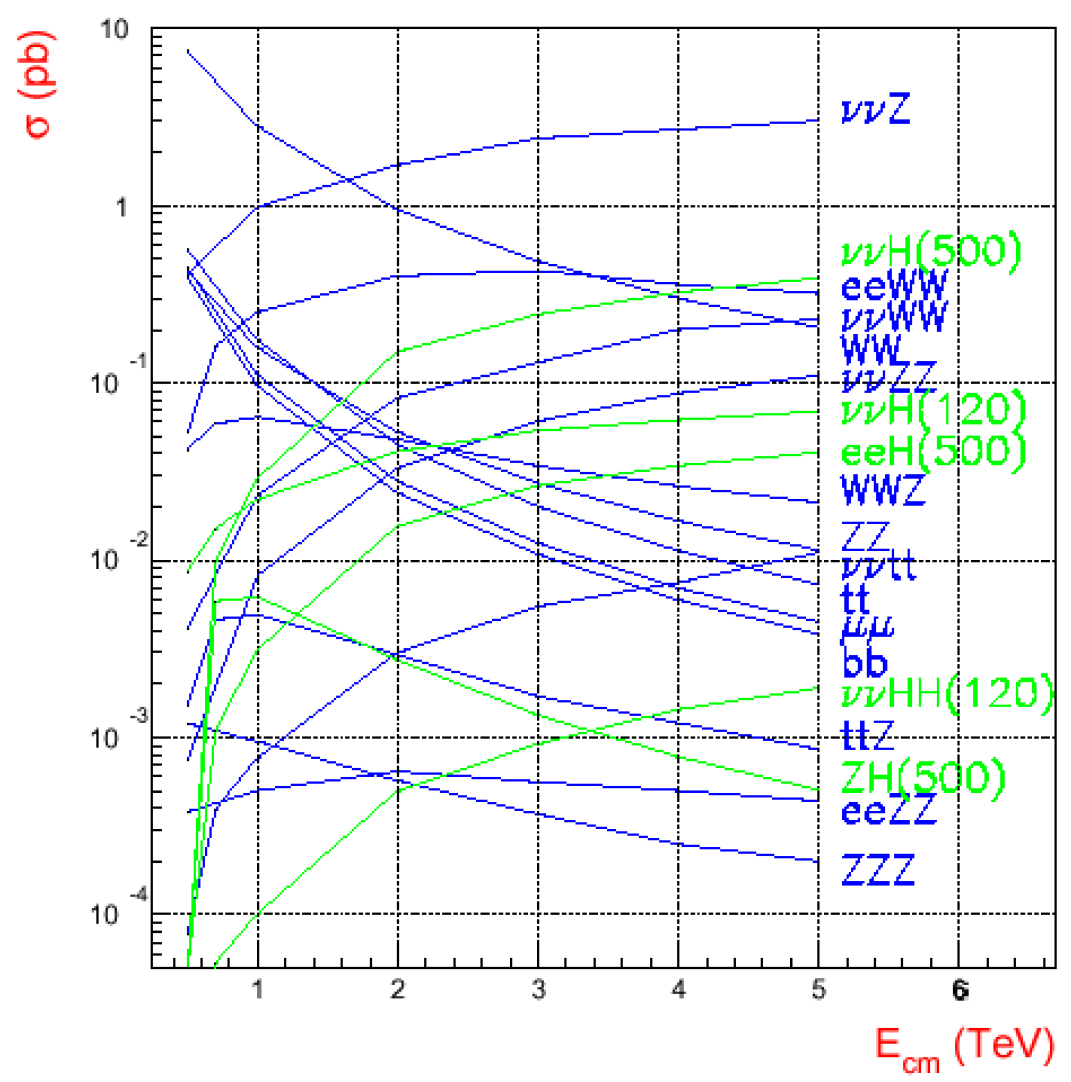

Figure 1.2. Cross sections vs. center of mass energy for various processes at a lepton collider [1]. 
Presently there are two candidates for a next generation multi-TeV lepton collider: a $\mu^{+} \mu^{-}$circular collider, or an $\mathrm{e}^{+} \mathrm{e}^{-}$linear collider. Muons have two distinct advantages over electrons. The first is that when accelerated, muons lose less energy to synchrotron radiation than electrons; the instantaneous power radiated being given by $[9]$ :

$$
P=\frac{e^{2} c E^{4}}{6 \pi \epsilon_{0}\left(m c^{2}\right)^{4} \rho^{2}}
$$

where $e$ is the charge of the muon, $c$ is the speed of light, $E$ is the total energy of the muon, $\epsilon_{0}$ is the vacuum permittivity, $m$ is the mass of the muon, and $\rho$ is the bending radius. It can be seen that the radiated power goes as the inverse mass to the fourth power. Because $m_{e} / m_{\mu} \simeq 5 \times 10^{-3}$, a Muon Collider could be built in a ring and is therefore much more compact than an electron collider, which must be straight.

The second advantage is that at energies of $\sim \mathrm{TeV}$, beamstrahlung radiation (due to the electric fields of colliding beams) is much smaller for muons than electrons, meaning the energy spread of a muon beam $\left(\Delta E / E \sim 10^{-3}\right)$ is significantly smaller than that of an electron beam. This can be seen in Fig. 1.3.

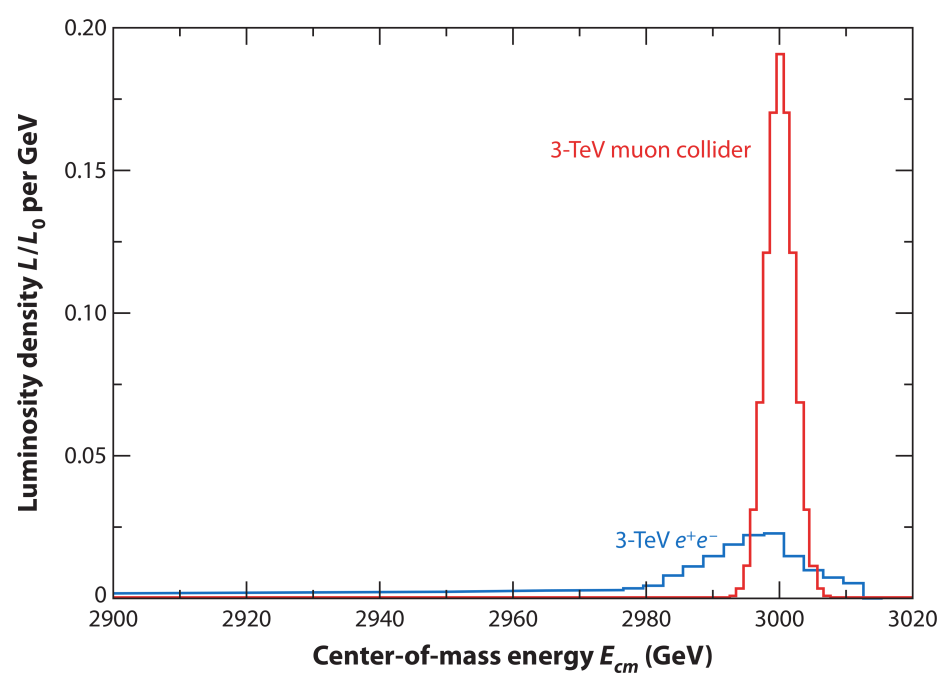

Figure 1.3. Luminosity density comparison between a $3 \mathrm{TeV}$ Muon Collider (red) and a $3 \mathrm{TeV} e^{+} e^{-}$collider (blue) [10].

A Muon Collider complex could look something like that shown in Fig. 1.4. A 
high power proton source is needed to produce the desired number of muons $\left(2 \times 10^{12}\right.$ muons per bunch would require $\sim 2 \times 10^{14}$ protons on target with a total power of $4 \mathrm{MW}$ ) (see Fig. 1.5) [11].

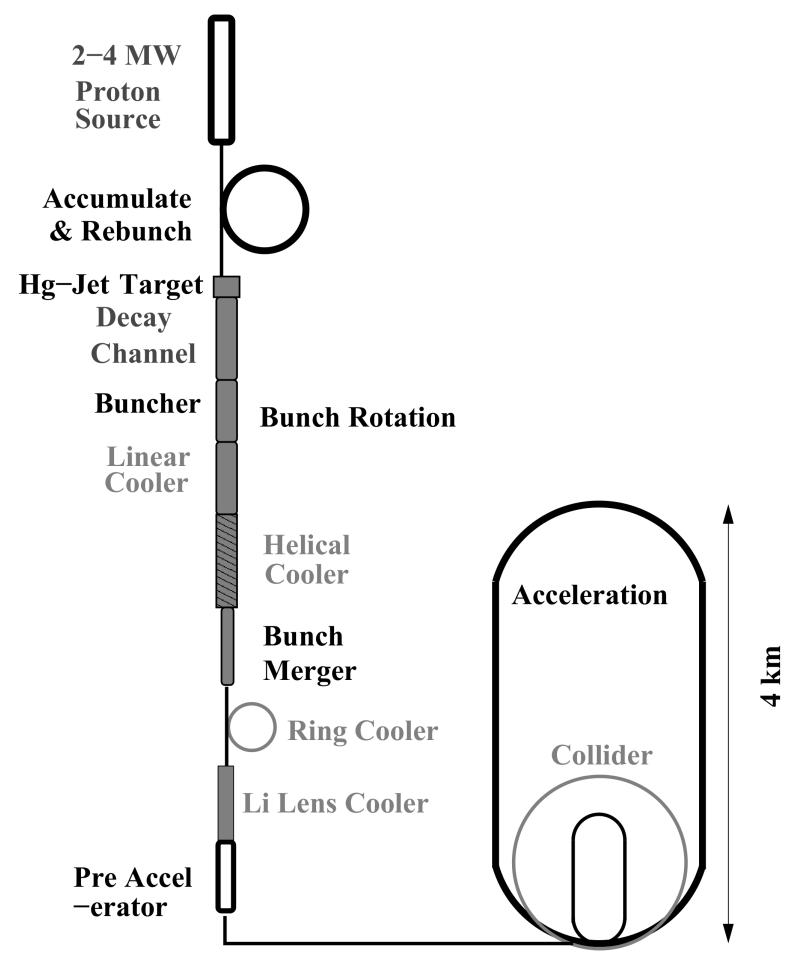

Figure 1.4. Possible schematic for a Muon Collider [11].

After being accumulated and bunched, the protons collide with a liquid mercury jet target where they produce pions. A successful demonstration of this technology was performed by the MERIT experiment, in which a liquid mercury jet housed in a $15 \mathrm{~T}$ solenoid was hit by a proton beam of up to $4 \mathrm{MW}$ (see Fig. 1.6) [12].

These pions are created with a huge phase space and must be captured in strong magnetic fields $(\sim 20 \mathrm{~T})$. A solenoid capture system to accomplish this has been designed [13]. Once the pions are captured they decay into muons, which are then bunched and phase rotated. This is a multi step process, depicted in Fig. 1.7. The muons begin with a large energy spread and small time spread. They are allowed to drift so that the higher and lower energy muons separate (a larger time spread, 


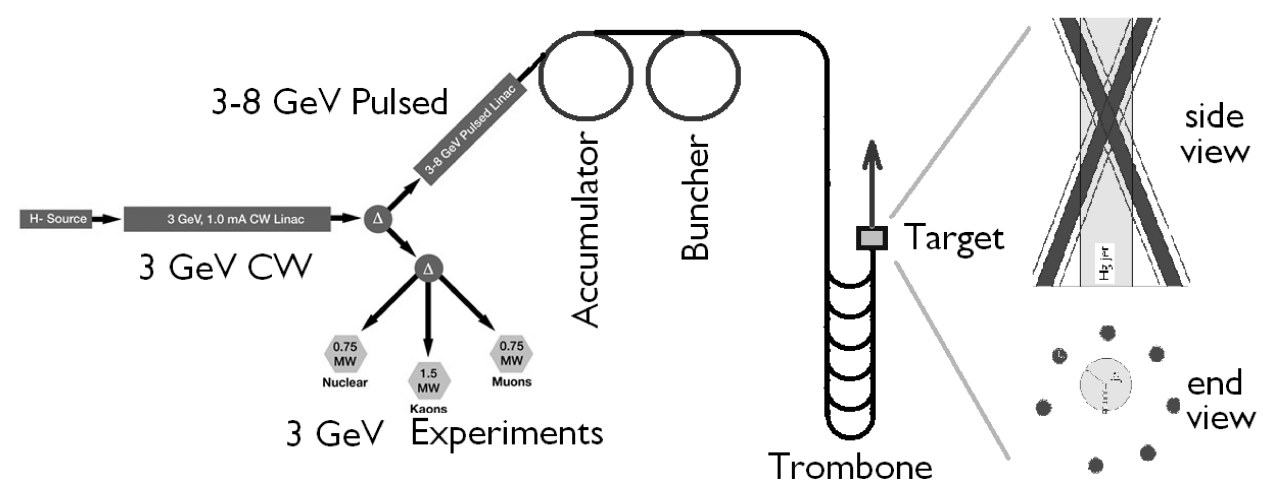

Figure 1.5. A potential proton driver and target system for a Muon Collider based on an upgraded Project X [1].

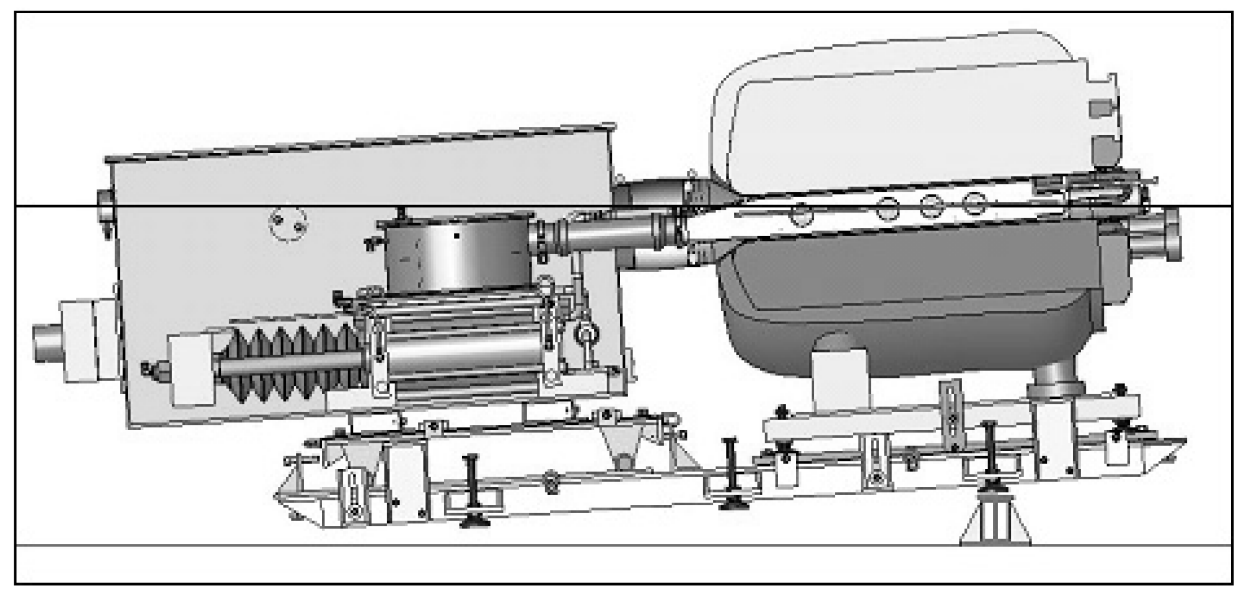

Figure 1.6. A schematic of the MERIT experiment. The liquid mercury injection system (on the left) shoots a jet of mercury into the solenoid (on the right) where a beam of protons hits it at a $67 \mathrm{mrad}$ angle [12]. 
while maintaining the same energy spread). RF cavities are then used to bunch the beam (due to the sinusoidal electric field), which maintains the time and energy spread. Finally, additional RF cavities are used to decrease the energy spread while increasing the time spread slightly (which is accomplished through proper phasing of the $\mathrm{RF}$ with respect to bunch timing). A simulation of the phase rotation is shown in Fig. 1.8. Notice the large energy and small time spreads before, and small energy and large time spreads after. The 12 most intense bunches are enclosed in the box in the right plot.

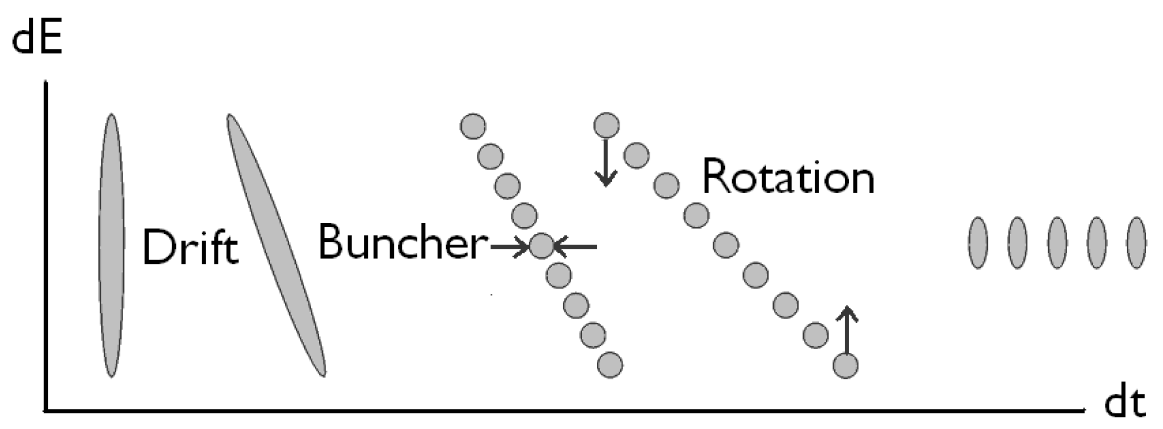

Figure 1.7. Conceptual plot showing the phase rotation process for a Muon Collider $[1]$.

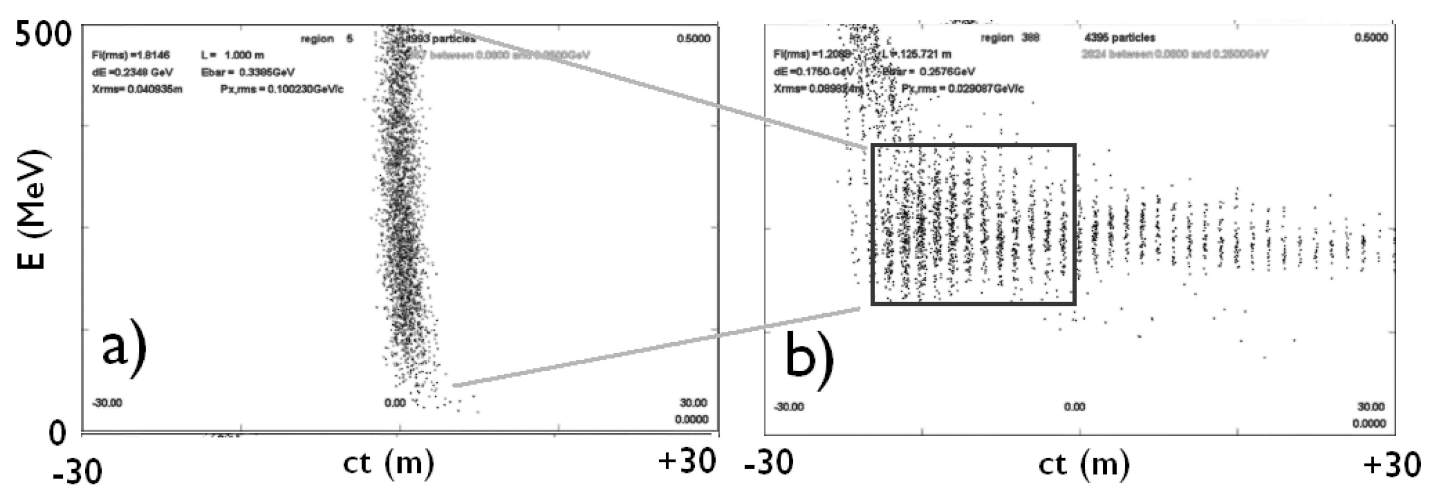

Figure 1.8. The results of a simulation of particle energy and time spread before (left) and after (right) the phase rotation [1].

Many orders of magnitude of emittance reduction ("cooling") is needed to get the muons to the desired emittance before they can be sent to the final acceleration and collider. This is accomplished in multiple steps using a technique called ionization 
cooling. A simulation of the longitudinal versus transverse emittance followed through these steps is shown in Fig. 1.9. It can be seen that the initial phase rotation and transverse (4D) cooling are required for a Neutrino Factory front end. Table 1.1 lists the $\mathrm{RF}$ requirements for the bunching, phase rotation, and 4D cooling.

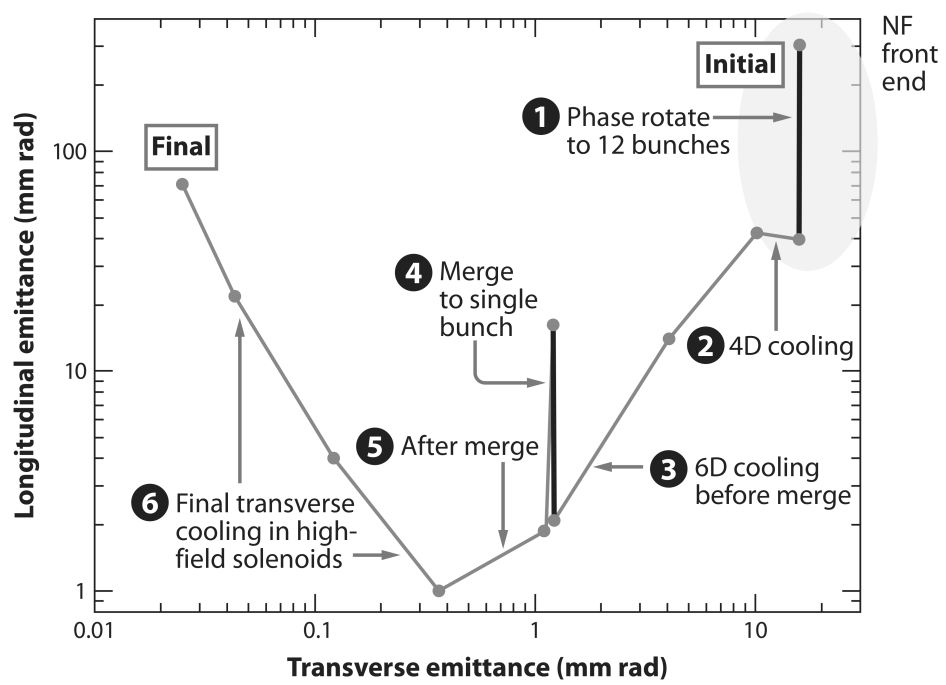

Figure 1.9. Cooling simulation for a possible Muon Collider cooling-channel [10]. 
Table 1.1. RF requirements for a Neutrino Factory [14].

\begin{tabular}{|c|c|c|c|}
\hline Section & Parameter & Value & Units \\
\hline \multirow[t]{5}{*}{ Buncher } & $p_{\text {muon }}$ & 233 & $\frac{\mathrm{MeV}}{\mathrm{c}}$ \\
\hline & $f$ & $319.6-233.6$ & $\mathrm{MHz}$ \\
\hline & $E$ & $4-7.5$ & $\frac{\mathrm{MV}}{\mathrm{m}}$ \\
\hline & $L$ & $0.4-0.5$ & $\mathrm{~m}$ \\
\hline & $B$ & 1.5 & $\mathrm{~T}$ \\
\hline \multirow[t]{4}{*}{ Rotator } & $p_{\text {muon }}$ & 230 & $\frac{\mathrm{MeV}}{\mathrm{c}}$ \\
\hline & $f$ & $230.2-202.3$ & $\mathrm{MHz}$ \\
\hline & $E$ & 12 & $\frac{\mathrm{MV}}{\mathrm{m}}$ \\
\hline & $L$ & 0.5 & $\mathrm{~m}$ \\
\hline \multirow[t]{6}{*}{ Cooler } & $p_{\text {muon }}$ & 230 & $\frac{\mathrm{MeV}}{\mathrm{c}}$ \\
\hline & f & 201.25 & $\mathrm{MHz}$ \\
\hline & $E$ & 15 & $\frac{\mathrm{MV}}{\mathrm{m}}$ \\
\hline & $L$ & 0.5 & $\mathrm{~m}$ \\
\hline & $B$ & 2.8 & $\mathrm{~T}$ \\
\hline & $N_{\text {muons }}$ & $10^{11}$ & $\frac{1}{\text { bunch }}$ \\
\hline
\end{tabular}


1.1.2 Neutrino Factory. Neutrinos have provided perhaps the most diverse source of new results in particle physics over the last decade. There are three known flavor eigenstates and three known mass eigenstates of neutrinos [15]. They are related to each other through a unitary matrix consisting of three mixing angles, $\theta_{12}, \theta_{23}$ and $\theta_{13}$, and one phase angle, $\delta$ :

$$
\left(\begin{array}{c}
\nu_{e} \\
\nu_{\mu} \\
\nu_{\tau}
\end{array}\right)=\left(\begin{array}{ccc}
c_{13} c_{12} & c_{13} s_{12} & s_{13} e^{-i \delta} \\
-c_{23} s_{12}-s_{13} s_{23} c_{12} e^{i \delta} & c_{23} c_{12}-s_{13} s_{23} s_{12} e^{i \delta} & c_{13} s_{23} \\
s_{23} s_{12}-s_{13} c_{23} c_{12} e^{i \delta} & -s_{23} c_{12}-s_{13} c_{23} s_{12} e^{i \delta} & c_{13} c_{23}
\end{array}\right)\left(\begin{array}{c}
\nu_{1} \\
\nu_{2} \\
\nu_{3}
\end{array}\right)
$$

where $s_{i j}=\sin \theta_{i j}$ and $c_{i j}=\cos \theta_{i j}$. Neutrino oscillation experiments have constrained the values of this matrix to be:

$$
U \approx\left(\begin{array}{ccc}
0.8 & 0.5 & ? \\
0.4 & 0.6 & 0.7 \\
0.4 & 0.6 & 0.7
\end{array}\right)
$$

The current world average sets $\sin ^{2} 2 \theta_{13}=0.098 \pm 0.013$ [16]. No measurements of $\delta$ have been published to date.

It is now known that neutrinos have mass, however the absolute values are not known. As a neutrino beam propagates through matter, its evolution is given by [17]:

$$
i \frac{d \nu_{\alpha}}{d t}=\sum_{\beta} \frac{1}{2 E_{\nu}}\left[\Delta m_{31}^{2} U_{\alpha 3} U_{\beta 3}^{*}+\Delta m_{21}^{2} U_{\alpha 2} U_{\beta 2}^{*}+A \delta_{\alpha \ell} \delta_{\beta \ell}\right] \nu_{\beta}
$$

where $\Delta m_{i j}^{2}=m_{i}^{2}-m_{j}^{2}, U_{i j}$ is a mixing matrix element, $A /\left(2 E_{\nu}\right)$ is the amplitude for coherent forward charged current scatter of $\nu_{e}$ on electrons, and $\delta_{i j}$ is a delta function. Neutrino oscillation experiments have determined the magnitude of $\Delta m_{21}^{2}$, but not sign of $\Delta m_{31}^{2}$. Equation 1.7 can be solved numerically to predict the probability of a neutrino of flavor $\alpha$ and energy $E_{\nu}$ oscillating to flavor $\beta$ as it travels distance $L$. Therefore a Neutrino Factory could be optimized for sets of $E_{\nu}$ and $L$ in order to study specific parameters. For the case of a $25 \mathrm{GeV}$ Neutrino Factory, detectors at baselines of $2500-5000 \mathrm{~km}$ and $7000-8000 \mathrm{~km}$ would be ideal.

There is also good reason to believe that neutrinos violate charge-parity (CP) conservation and that they are their own antiparticles (Majorana). Of these remaining 
questions, a Neutrino Factory could shed light on CP violation, the mass hierarchy, and mixing angles.

The front end of a Muon Collider could be the same as the front end of a Neutrino Factory. Figure 1.10 shows a schematic for a Neutrino Factory. The proton driver, target, buncher, and phase rotation sections could serve for both. Less overall (perhaps even just 4D) cooling is required for a Neutrino Factory than a Muon Collider. After cooling, the beams are sent to a series of recirculating linear accelerators (RLAs) and optionally a fixed-field alternating-gradient (FFAG) accelerator. Finally each beam $\left(\mu^{+}\right.$and $\left.\mu^{-}\right)$enters a ring with long straight segments in which the muons decay and send neutrinos toward the detectors.

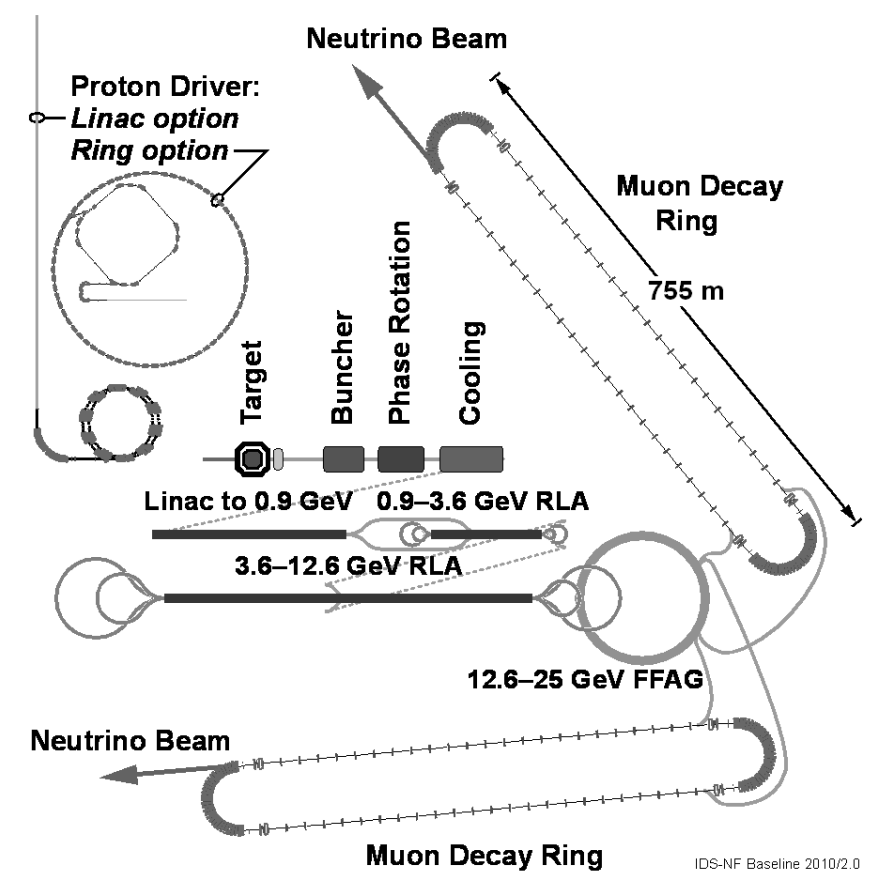

Figure 1.10. Schematic diagram of a Neutrino Factory.

Tables 1.2 and 1.3 show some typical parameters for Muon Collider and Neutrino Factory schemes. 
Table 1.2. Proposed Muon Collider parameters [1].

\begin{tabular}{llcc}
\hline \hline Parameter & Unit & \multicolumn{2}{c}{ Value } \\
\hline Center of mass energy & $\mathrm{TeV}$ & 1.5 & 3 \\
Luminosity & $10^{34} \mathrm{~cm}^{-2} \mathrm{~s}^{-1}$ & 1.25 & 4.4 \\
Proton driver beam power & $\mathrm{MW}$ & 4 & 3.2 \\
Muons per bunch & $10^{12}$ & 2 & 2 \\
Muon beam power (both beams) & $\mathrm{MW}$ & 7.2 & 11.5 \\
Normalized rms emittance $\epsilon_{x, y}$ & $\mu \mathrm{m}$ & 25 & 25 \\
Normalized rms emittance $\epsilon_{z}$ & $\mu \mathrm{m}$ & 72,000 & 72,000 \\
Repetition rate & $\mathrm{Hz}$ & 15 & 12 \\
\hline
\end{tabular}

Table 1.3. Propose Neutrino Factory parameters [1].

\begin{tabular}{lcc}
\hline \hline Parameter & Unit & Value \\
\hline Muon energy & $\mathrm{GeV}$ & 25 \\
Muon decays in $10^{7} \mathrm{~s}$ & & $10^{21}$ \\
Distance to intermediate baseline detector & $\mathrm{km}$ & $2500-5000$ \\
Distance to far baseline detector & $\mathrm{km}$ & $7000-8000$ \\
Normalized transverse acceptance & $\mathrm{mm}$ & 30 \\
Normalized longitudinal acceptance & $\mathrm{mm}$ & 150 \\
\hline
\end{tabular}

1.1.3 Cooling. Stochastic cooling (a feedback method in which a particle's motion 
is corrected by a kicker), electron cooling (a cold co-moving electron beam), or other traditional cooling methods do not work for muons due to their short lifetime $(2.2 \mu \mathrm{s})$. Ionization cooling appears to be the only method that provides the desired emittance reduction in the required time $[18,19]$. The technique of transverse ionization cooling is illustrated in Fig. 1.11. A particle passes through an absorbing material, losing momentum in the transverse and longitudinal directions by ionizing the absorbing material. Momentum is then replaced in the longitudinal direction by an RF cavity. There are two competing effects involved. The net loss of momentum in the transverse direction decreases the emittance, while multiple Coulomb scattering in the material increases the emittance. The choice of material must be such that the latter is smaller than the former. The change in normalized transverse emittance over path length is given approximately by Eq. 1.8, where $\beta=v / c, E_{\mu}$ is the energy of the muon in $\mathrm{GeV}$, $\beta_{\perp}$ is the optical beta function in the magnetic channel in meters, $m_{\mu}$ is the mass of the muon in $G e V / c^{2}$, and $X_{0}$ is the radiation length of the absorber in meters [20]. The negative term in Eq. 1.8 is the cooling term and the positive term is the heating term:

$$
\frac{d \epsilon_{n}}{d s} \approx-\frac{1}{\beta^{2}}\left\langle\frac{d E_{\mu}}{d s}\right\rangle \frac{\epsilon_{n}}{E_{\mu}}+\frac{1}{\beta^{3}} \frac{\beta_{\perp}(0.014)^{2}}{2 E_{\mu} m_{\mu} X_{0}}
$$

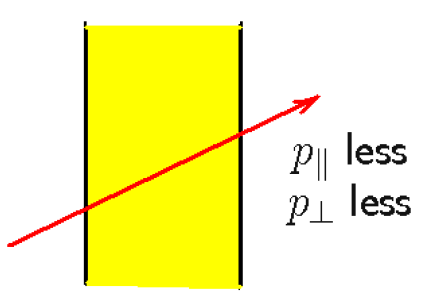

Material

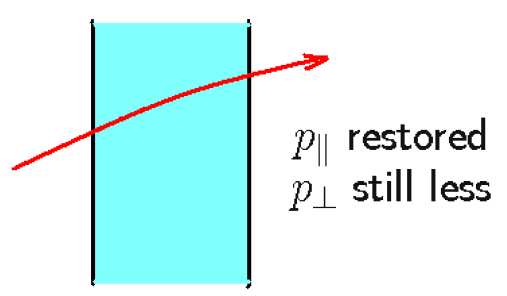

Acceleration

Figure 1.11. Diagram of transverse ionization cooling. A particle (red) passes through an absorber (yellow), losing energy. It is then reaccelerated in the longitudinal direction by an RF cavity (cyan) [1].

While this provides cooling in the transverse dimension, it does not cool in the longitudinal dimension. Transverse cooling alone is not sufficient to achieve the desired luminosity, so a process called emittance exchange must be employed. The 
concept is to decrease $\delta E / E$ by making high energy particles pass through more absorbing material than low energy particles. Figure 1.12 shows three possible methods. All three use a magnet to bend the path of the beam. This produces dispersion; the path length of higher energy particles will be longer than that of lower energy particles. This will also increase the transverse size of the beam. In the first method, the beam passes through a wedge absorber in which higher energy particles pass through more material. This has an overall effect of reducing the energy spread (longitudinal emittance) while increasing the transverse size (transverse emittance) of the beam. The second method combines the magnet and absorber, producing the same result. The third method uses angular dispersion to make the higher energy particles pass through a slab of absorber (placed between two magnets) at an angle, thereby increasing the path length.

All three methods have been used to simulate cooling-channels and each cools in all six phase space dimensions. Figure 1.13 shows a model of each. The so-called Guggenheim RFOFO channel is shown in the top left [21]. The first example of emittance exchange as mentioned above is employed here. RF cavities are placed inside pairs of opposite polarity solenoids that provide transverse focusing. The channel is wrapped in the shape of a helix to provide dispersion, while wedge absorbers are placed between RF cavities at beta function minima. This method uses vacuum cavities in solenoidal magnetic fields up to $\approx 7 \mathrm{~T}$.

The second method is shown in the top right of Fig. 1.13 and is called the Helical Cooling Channel (HCC) [22]. In this geometry, offset solenoids surround RF cavities and create a helical beam trajectory. In this case, the beampipe is filled with high pressure hydrogen gas to act as the ionization medium. Particles with larger momentum pass through more absorbing material than lower momentum particles. This method uses high pressure gas filled cavities in solenoidal magnetic fields up to 


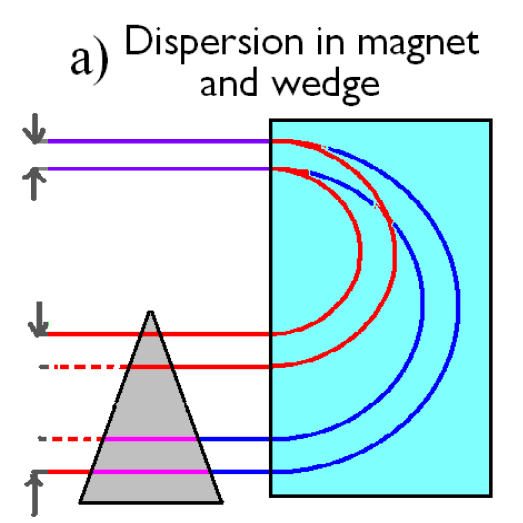
b) Path length differences in magnet

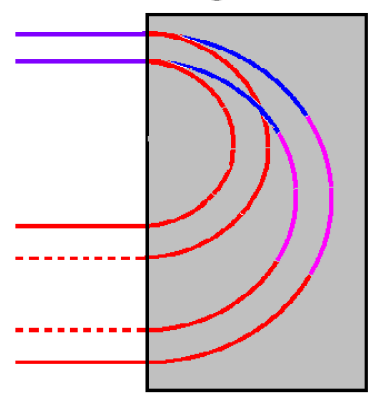

c) Angular dispersion and path lengths in slab

Figure 1.12. Three examples of emittance exchange. a) Dispersion in a magnet (cyan) and an absorbing material wedge (gray). b) Dispersion in a magnet filled with an absorbing material. c) Angular dispersion in two magnets with an absorbing slab between [1].
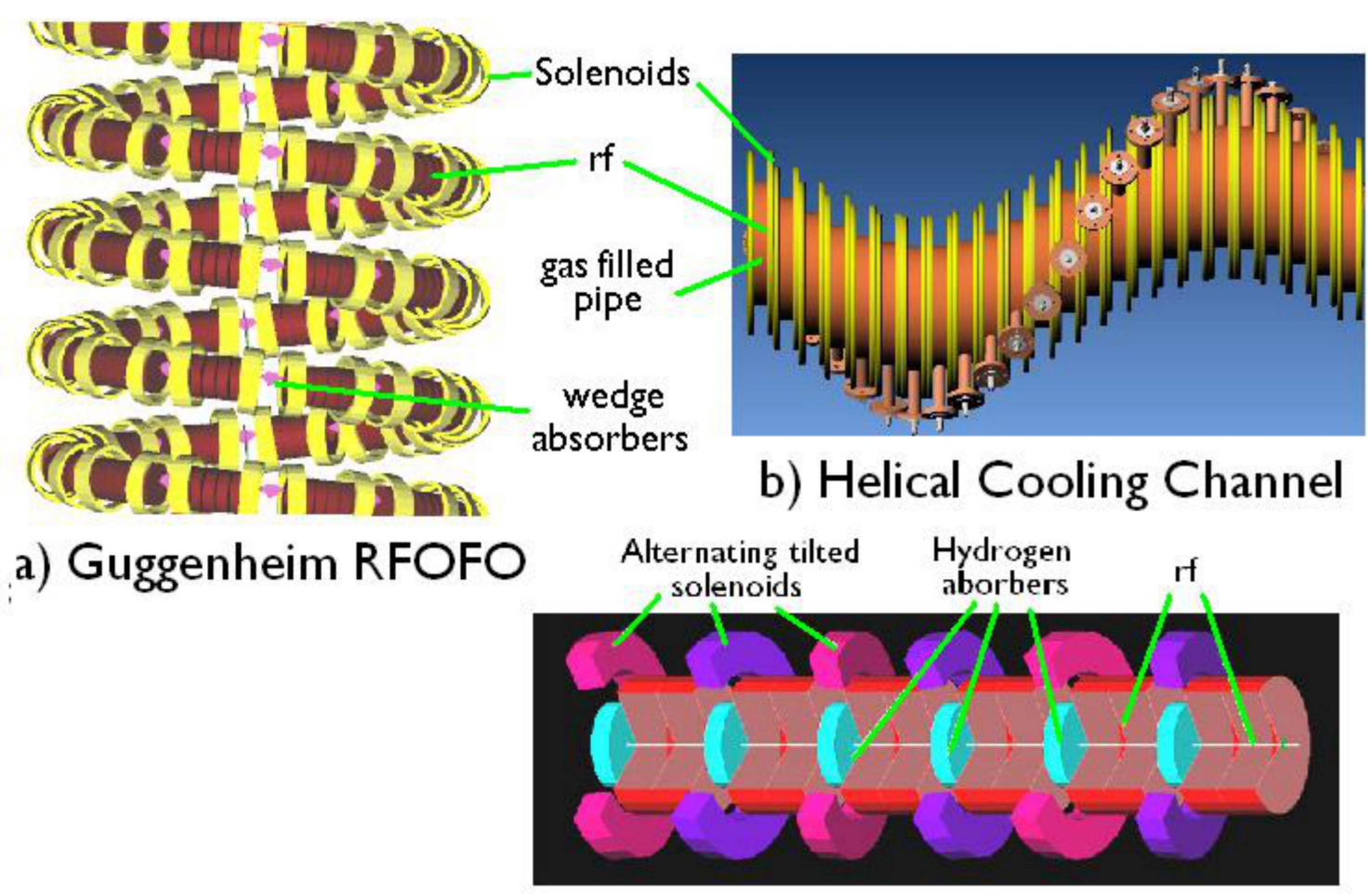

c) Helical FOFO Snake

Figure 1.13. Three possible 6D cooling-channels. Top left is the Guggenheim RFOFO (the yellow rings are solenoids, the brown pillboxes are RF cavities), top right is the helical, and bottom is the helical FOFO snake (the purple and magenta toroids are alternating tilted solenoids, the cyan disks are absorbers, and the red pillboxes are RF cavities) [1]. 
$14 \mathrm{~T}$.

The final method is shown in the bottom of Fig. 1.13 and is called the Helical FOFO Snake [23]. This channel uses alternating polarity tilted solenoids around disk absorbers to provide angular dispersion. The previous two methods work for only one sign of muons, while the Helical FOFO Snake works for both because of the phasing of the beam at the alternating polarity tilted solenoids.

The bunches are merged into one, and more 6D cooling is applied. The final transverse cooling is done in high field solenoids. This is accomplished in many stages of absorber-filled solenoids and RF cavities. The field strength of the solenoids must be $30-50$ T. From there the muon beam is sent to the final acceleration.

For the 4D cooling and in each 6D cooling-channel, RF cavities are required to operate in very strong magnetic fields. This is one of the main technological challenges in building a muon accelerator. As will be seen later, traditional vacuum cavities break down in such conditions, and so new techniques are required in order to circumvent this.

\subsection{Theory}

1.2.1 Breakdown in an RF Field. In any accelerator RF cavity, electrons emitted from the surface of the metal and accelerated by the electric field ("dark current") are present. This is quantum tunneling of electrons through the potential barrier at the surface of the metal (work function). There are two main sources of dark current: reduction of the potential barrier due to the electric field at the surface, or thermionic emission. The Fowler-Nordheim equation gives the current density (in $\mathrm{A} / \mathrm{m}^{2}$ ) due to the surface electric field [24]:

$$
j=\frac{A_{F N}\left(\beta E_{\text {surf }}\right)^{2}}{\phi} e^{-\frac{B_{F N} \phi^{3 / 2}}{\beta E_{\text {surf }}}}
$$

where $A_{F N}=1.54 \times 10^{6} \mathrm{eV} \mathrm{A} / \mathrm{MV}^{2}, \beta$ is the ratio of the local electric field at the 
emitter to the average surface field, $E_{\text {surf }}$ is the surface electric field, $\phi$ is the work function of the material (for copper, $\phi=4.7 \mathrm{eV}$ ), and $B_{F N}=6830 \mathrm{MV} /\left(\mathrm{m}(\mathrm{eV})^{3 / 2}\right.$ ). Measurements for one $\mathrm{R} \& \mathrm{D}$ cavity indicate $\beta \approx 184$, meaning the local field at an emitter ranges $1.8-9.2 \frac{\mathrm{GV}}{\mathrm{m}}$ (for $E_{\text {surf }}=10-50 \frac{\mathrm{MV}}{\mathrm{m}}$ ) [24]. This results in a current density of $4.2 \times 10^{-5}-1.4 \times 10^{10} \frac{\mathrm{A}}{\mathrm{m}^{2}}$ (the size of a typical emitter is $10^{-14} \mathrm{~m}^{2}$ ). The thermionic emission current density is given by [24]:

$$
j=A T^{2} e^{-\frac{\phi}{k_{B} T}}
$$

where $A=1.2 \times 10^{6} \mathrm{~A} /(\mathrm{K} \mathrm{m})^{2}, T$ is the temperature, and $k_{B}$ is Boltzmann's constant. For emitter temperatures below $5000 \mathrm{~K}$, the current density from Fowler-Nordheim emission is orders of magnitude larger than that from thermal emission.

When the current density gets sufficiently high, an arc may form between the end plates of the cavity, shorting it and causing breakdown. Simulations of dark current show that the presence of an axial (solenoidal) magnetic field greatly focuses the emitted electrons traversing the length of the cavity [25]. An example is shown in Fig. 1.14. It can be seen that even a $0.5 \mathrm{~T}$ magnetic field has a significant focusing effect on the emitted electrons.

Experiments have been performed with both open cell and pillbox cavity geometries, using various metallic materials, to map the performance of vacuum RF cavities in external magnetic fields [24-28]. Data on the degradation of the maximum achievable accelerating gradient vs. applied external magnetic field are shown in Fig. 1.15.

In order to mitigate breakdown of an RF cavity in an external magnetic field, it was proposed to fill the cavity with a high pressure gas to prevent the electrons from forming an arc [29]. If the gas used is hydrogen, it provides the additional benefit of cooling a beam of muons that pass through the cavity. The gas reduces the mean 

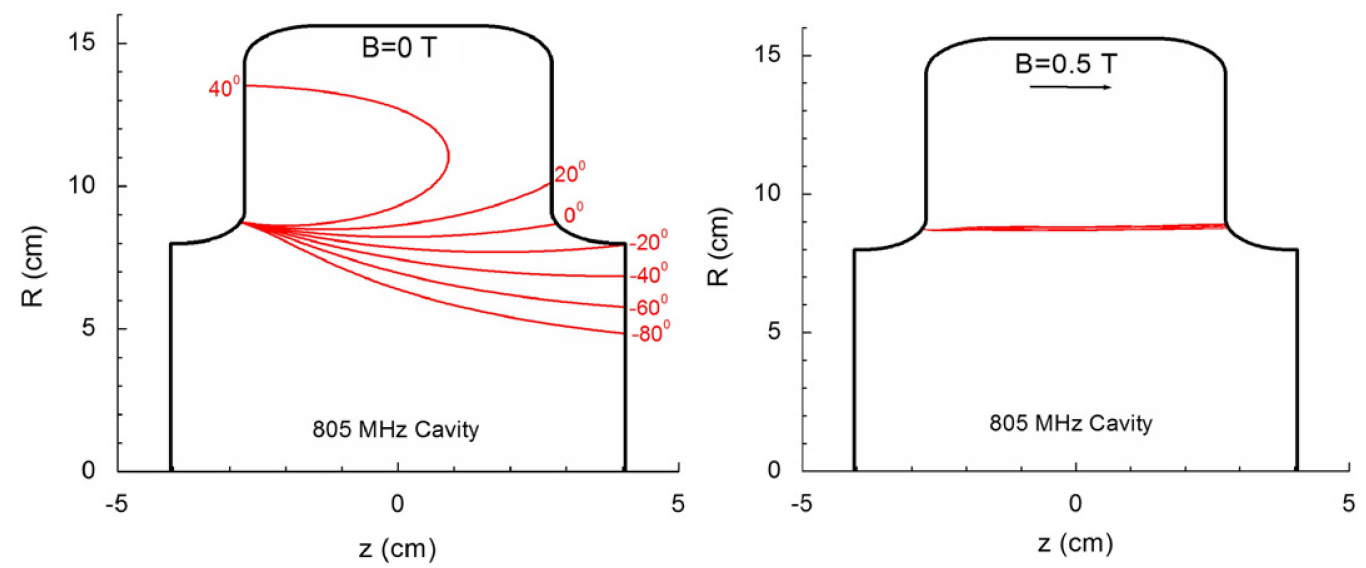

Figure 1.14. Simulation of the magnetic focusing effect in an RF cavity. The path of the electron (red) traversing the cavity is shown, with the phase it was emitted relative to the peak electric field labeled. On the left there is no external axial magnetic field. On the right there is a $0.5 \mathrm{~T}$ axial field [25].

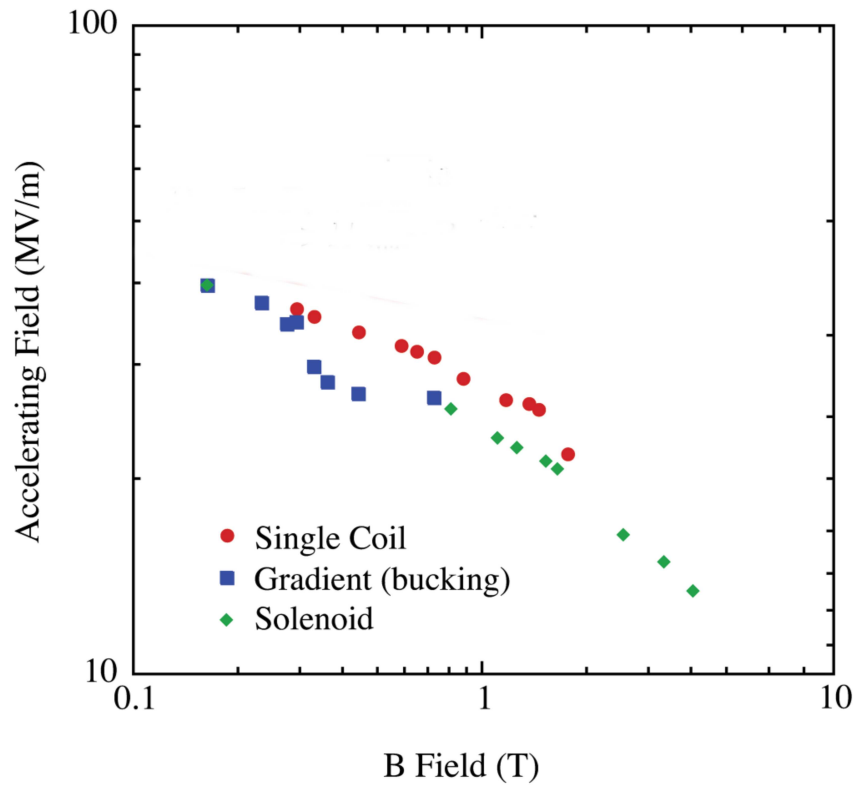

Figure 1.15. Maximum accelerating field in a pillbox cavity vs. external magnetic field. Different modes of magnet operation are shown [26]. 
free path, $\ell$, of the electrons by causing them to lose energy through collisions with gas molecules. If, $e E \ell<$ the ionization energy of the gas $(E$ is the applied electric field), the electrons never gain the energy required to ionize the gas and produce more electrons. The denser the gas, the more collisions an electron would make, and so the maximum achievable electric field in the cavity should increase with gas pressure. When an electron gains enough energy to ionize the gas, a cascade of electrons can be produced, called a Townsend Avalanche [30]. The current in the gas is given by:

$$
I=I_{0} e^{\alpha d}
$$

where $I_{0}$ is the initial electron current at the source, $d$ is the electrode separation, and $\alpha$ is the first Townsend coefficient. It is convention to normalize the electric field by the pressure $(E / P=X)$ because the average energy gained between collisions is constant for a constant $X$. Figure 1.16 shows $\alpha$ normalized to pressure vs. $X$.

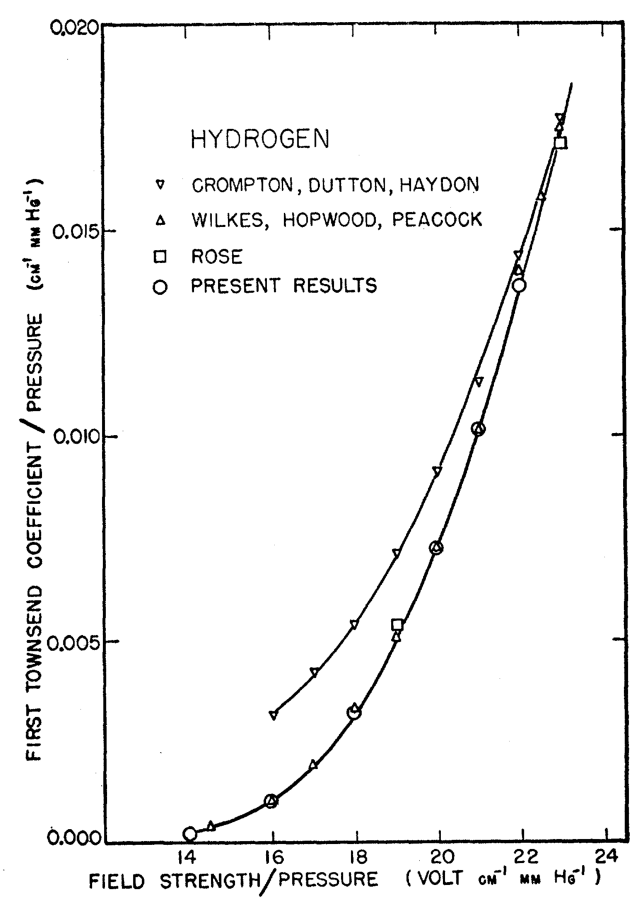

Figure 1.16. The first Townsend coefficient normalized to pressure vs. $X$ [30].

This avalanche causes the cavity to break down. Measurements of the limit at which increasing the gas pressure no longer prevents breakdown have been made [31]. 
This boundary for gas breakdown falls between 14 and $20(\mathrm{~V} / \mathrm{cm}) / \mathrm{mmHg}$, or in more applicable units, $1.06-1.51(\mathrm{MV} / \mathrm{m}) / \mathrm{atm}(0.0724-0.103(\mathrm{MV} / \mathrm{m}) / \mathrm{psi})$.

Studies have shown that filling an RF cavity with a high pressure gas does in fact prevent breakdown in strong magnetic fields. An $805 \mathrm{MHz}$ high pressure pillbox test cell fitted with electrodes to enhance the electric field shows great improvement over vacuum cavities for a variety of electrode materials and gas species [32,33]. Figures 1.17 and 1.18 summarize the results.

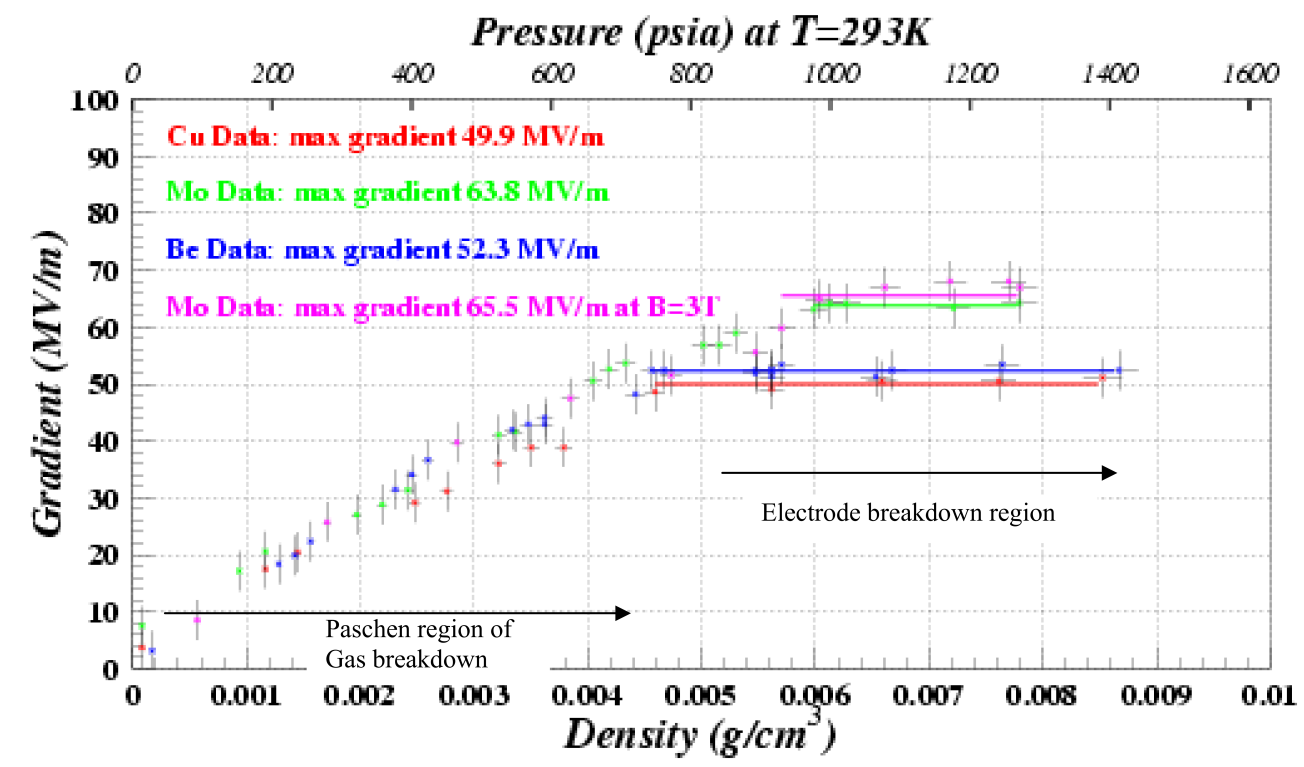

Figure 1.17. Maximum gradient vs. pressure for different electrode materials [32].

Figure 1.17 shows the maximum gradient vs. hydrogen gas pressure for various electrode materials. It can be seen that the electric field increases linearly with pressure up to a certain pressure (this is called the Paschen Region), at which point the breakdown field is determined by electrode material (this is called the Metallic Region). The exact mechanism that causes this material dependence is not known, although it is believed that for sufficiently large electric fields, the surface of the metal may actually melt, and properties such as the work function of the metal contribute significantly [34]. Note that there is virtually no difference in maximum gradient using a molybdenum electrode with and without a $3 \mathrm{~T}$ magnetic field. 


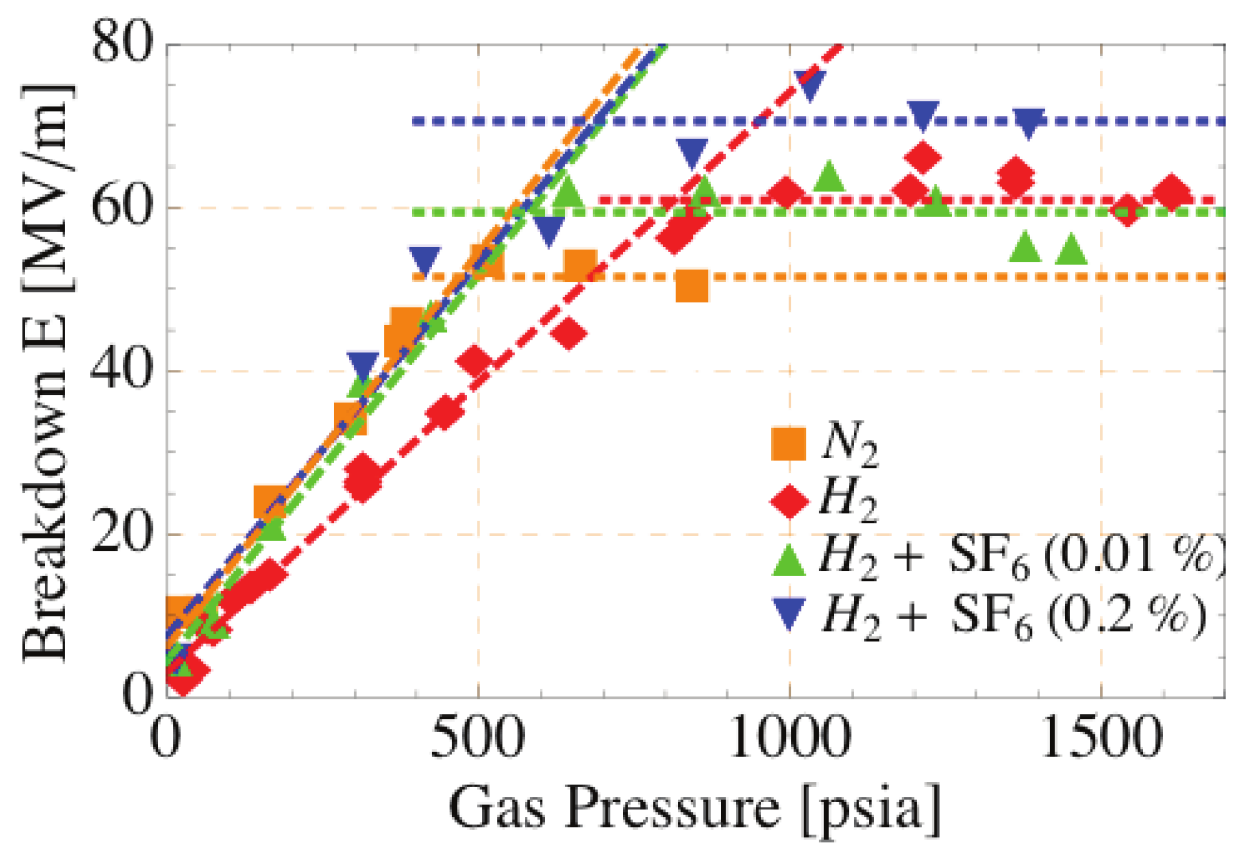

Figure 1.18. Maximum gradient vs. pressure for different gas species [33].

Figure 1.18 shows the maximum gradient vs. gas pressure for various gas species (using copper electrodes). The Paschen Region can be seen in this data set as well, with the parent gas species (hydrogen or nitrogen) affecting the slope and ultimately the maximum electric field. Doping the parent gas with an electronegative gas changes the slope and maximum field as well.

These encouraging results necessitate a more realistic test of a high pressure gas filled radio frequency (HPRF) cavity. As will be seen later, the plasma created by sending a beam of particles through the gas consumes RF power. The extent of this power consumption and methods to mitigate it must be explored before an HPRF cavity can be validated for use in a muon cooling-channel.

1.2.2 Plasma Formation. Charged particles passing through hydrogen gas will interact with the gas through ionizing and dissociative ionizing collisions. In this experiment those particles are protons, however in this respect there is very little difference between the interactions of protons and muons. The dominant process is 
single ionization [35]:

$$
p+\mathrm{H}_{2} \rightarrow p+\mathrm{H}_{2}^{+}+e^{-}
$$

Dissociative ionizing processes are also known to occur at the few percent level [35]:

$$
\begin{aligned}
& p+\mathrm{H}_{2} \rightarrow p+\mathrm{H}+\mathrm{H}^{+}+e^{-} \\
& p+\mathrm{H}_{2} \rightarrow p+\mathrm{H}+\mathrm{H}^{+*}+e^{-}
\end{aligned}
$$

The ionization electrons can have enough energy to ionize hydrogen as well. Again, the dominant process is ionization, with dissociative ionization possible as well [36]:

$$
\begin{aligned}
& e^{-}+\mathrm{H}_{2} \rightarrow \mathrm{H}_{2}^{+}+2 e^{-} \\
& e^{-}+\mathrm{H}_{2} \rightarrow H+\mathrm{H}^{+}+2 e^{-}
\end{aligned}
$$

These processes produce a plasma that is mostly $e^{-}$and $\mathrm{H}_{2}^{+}$. At high pressures of hydrogen background gas, the $\mathrm{H}_{2}^{+}$ions quickly interact to form $\mathrm{H}_{3}^{+}$(usually within one picosecond) via [37]:

$$
\mathrm{H}_{2}^{+}+\mathrm{H}_{2} \rightarrow \mathrm{H}_{3}^{+}+\mathrm{H}
$$

$\mathrm{H}_{3}^{+}$can be formed in an excited vibrational state, but quickly relaxes through collisions with $\mathrm{H}_{2}[38,39]$. Larger clusters of hydrogen can be formed through three-body collisions between the hydrogen ions and gas molecules [40]. The resulting hydrogen clusters can be dissociated through additional collisions. Eventually an equilibrium of the population of hydrogen ion clusters will be reached based on gas temperature and pressure.

$$
\mathrm{H}_{n-2}^{+}+\mathrm{H}_{2}+\mathrm{H}_{2} \leftrightharpoons \mathrm{H}_{n}^{+}+\mathrm{H}_{2} \quad(n=5,7,9, \ldots)
$$

For an incident particle of mass $M$ and momentum $M \beta \gamma c$, the maximum initial kinetic energy of an ionization electron is given by [41]:

$$
\epsilon_{\max }=\frac{2 m_{e}(c \beta \gamma)^{2}}{1+2 \gamma\left(m_{e} / M\right)+\left(m_{e} / M\right)^{2}}
$$


where $c$ is the speed of light, $\beta=v / c, v$ is the velocity of the incident particle, and $\gamma=\frac{1}{\sqrt{1-\beta^{2}}}$. In this experiment, $\gamma \simeq \sqrt{2}$, and the incident particle is a proton, so $m_{e} / M \approx 1 / 2000$. Eq. 1.19 reduces to $\epsilon_{\max }=2 m_{e} c^{2} \beta^{2} \gamma^{2}$. In this experiment $\epsilon_{\max } \simeq$ $1 \mathrm{MeV}$. For incident muons, this is still a good approximation up to $\gamma$ approaching 10. For muon cooling channels currently under consideration, $\gamma \simeq 2$.

Energy gained by electrons from the RF field is transferred to the surrounding gas through collisions. The electron thermalization time is given by:

$$
\tau_{e}=\frac{1}{\zeta_{e} \nu_{e}}
$$

where $\nu_{e}$ is the collision frequency and $\zeta_{e}$ is the fractional energy loss per collision. For the case of electrons with energy below the ionization level, rotational and vibrational collisions dominate, and $\zeta_{e} \sim 10^{-3}-10^{-2}$ [42]. The energy loss for an elastic collision is smaller, $\zeta_{e}=2 m_{e} /\left(m_{e}+m_{\mathrm{H}_{2}}\right) \approx 1 / 2000$. Assuming a Maxwellian distribution of electrons, the collision frequency over a pressure range of 300-1520 psi(20.4-103 atm) at $300 \mathrm{~K}$ is $7.2-36.6 \times 10^{12} \mathrm{~s}^{-1}$ [43]. This gives a maximum thermalization time of $0.28 \mathrm{~ns}$. Since the half period of $805 \mathrm{MHz}$ is $0.62 \mathrm{~ns}$, it is safe to assume the electrons are always in thermal equilibrium with the surrounding gas. The electrons then drift with the applied RF electric field.

The number of electron-ion pairs produced by the proton beam can be estimated. Using the density of hydrogen gas, $\rho$, the average energy required to ionize a hydrogen molecule, $W_{i}$, the energy loss per unit length of $400 \mathrm{MeV}$ protons in hydrogen (see Fig. 1.19) $\frac{d E}{d x}$, and the cavity length $h$, one gets:

$$
N_{\text {pairs }}=\frac{d E / d x \rho h}{W_{i}} \times N_{p}
$$

where $N_{p}$ is the measured number of protons incident on the cavity. The uncertainty in $N_{\text {pairs }}$ is determined by the beam current measurement. The rates of ionization and dissociative ionization for protons and electrons on hydrogen gives an uncertainty 
on the number of electrons and ions produced of $\sim 5 \%$.

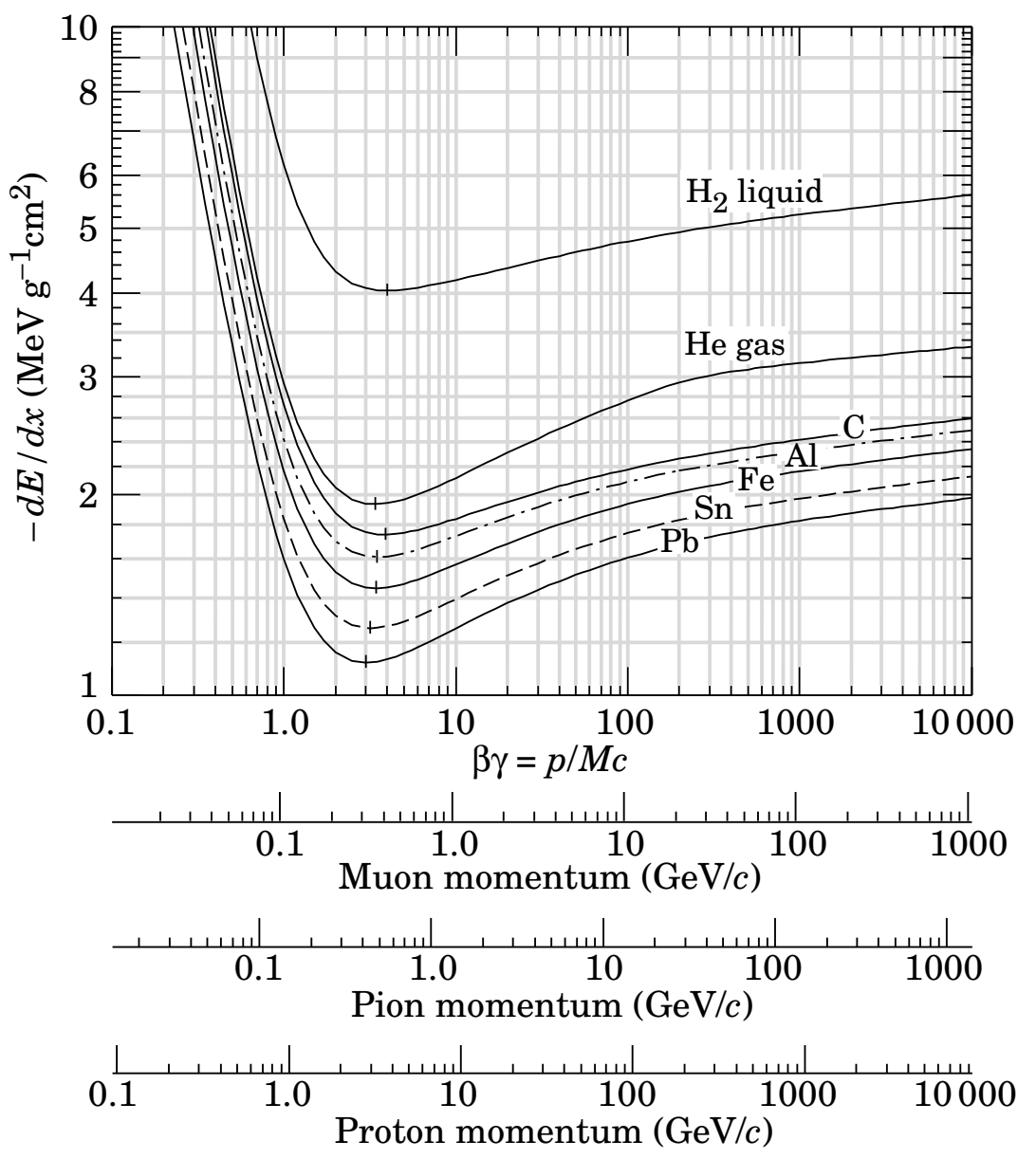

Figure 1.19. Mean energy loss rate versus particle momentum in various materials [41].

\subsubsection{Energy Loss.}

1.2.3.1 Beam Loading. A beam of charged particles passing through an RF cavity will produce an effect called beam loading. As a bunch of charge $+q$ passes through the accelerating gap along the axis of the cavity, a charge of $-q$ due to the image current will accumulate on the upstream end of the gap and a charge of $+q$ will accumulate on the downstream end of the gap (see Fig. 1.20). A voltage will thus 
be created across the gap, and for an infinitesimally short bunch will be [44]:

$$
V_{b}=\frac{q}{C}=\frac{q \omega_{r} R}{Q}
$$

where $C$ is the equivalent gap capacitance, $\omega_{r}$ is the resonant frequency of the cavity, $R$ is the shunt impedance, and $Q$ is the unloaded quality factor. This voltage opposes and reduces the accelerating voltage of the cavity; the voltage seen by the next bunch is thus reduced. The energy removed from the cavity by the bunch is [45]:

$$
W_{b}=\frac{1}{2} \frac{q^{2}}{C}=\frac{1}{2} \frac{R}{Q} \omega_{r} q^{2}=\frac{1}{2} q V_{b}
$$

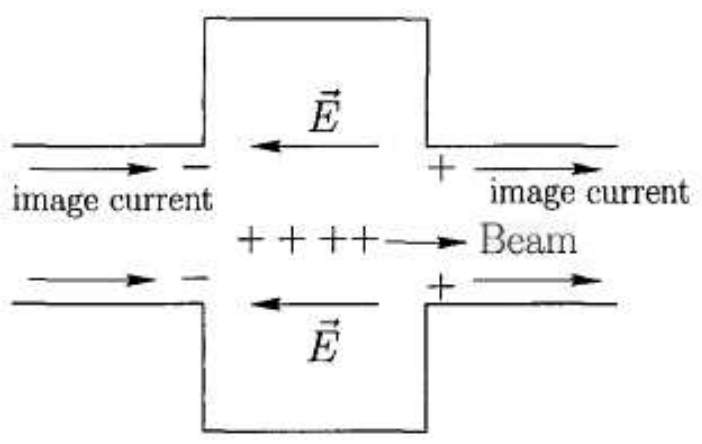

Figure 1.20. An illustration of beam loading [44]. The beam produces an image current, which leaves a positive charge on on the downstream end of the cavity and a negative charge on the upstream end of the cavity. The induced electric field opposes and lowers the accelerating voltage of the cavity.

The net voltage seen and energy received by a bunch are (respectively) [45]:

$$
\begin{aligned}
V & =V_{g} \sin \phi_{b}-\frac{1}{2} V_{b} \\
W & =q V_{g} \sin \phi_{b}-W_{b}=q\left(V_{g} \sin \phi_{b}-\frac{1}{2} V_{b}\right)
\end{aligned}
$$

where $V_{g}$ is the generator voltage and $\phi_{b}$ is the beam phase angle measured from the zero crossing of the RF wave.

1.2.3.2 Plasma Loading. A charged particle in an RF cavity absorbs power from the cavity. For the case of an HPRF cavity, the number of ionization electrons 
and positive ions produced by the beam is roughly given by Eq. 1.21. Because the electrons come into thermal equilibrium with the surrounding plasma in $\approx 0.1 \mathrm{~ns}$, they are confined to the cavity and drift with the electric field. The amount of energy such a charged particle absorbs is given by:

$$
\int P d t=\int q v E d t
$$

where $P$ is power, $q$ is the charge of the particle, $v$ is its drift velocity in the plasma, and $E$ is the applied electric field. The drift velocity is a function of the particle's mobility, $\mu$, and the electric field:

$$
v_{d r i f t}=\mu E
$$

The power loss in the HPRF cavity vs. time follows the drift velocity and electric field. The drift velocity of electrons in hydrogen gas has been well documented [46-52]. The mobility of electrons in hydrogen gas has also been well measured [53-59]. Figure 1.21 shows the drift velocity of electrons in hydrogen gas at $293 \mathrm{~K}$ vs. $E / P(X)$. The range of $X$ in the HPRF beam test is $0.636-11.6 \mathrm{~V} / \mathrm{cm} / \mathrm{mmHg}$. A useful conversion is $1 \mathrm{MV} / \mathrm{m} / \mathrm{psi}=193.4 \mathrm{~V} / \mathrm{cm} / \mathrm{mmHg}$.

The energy loss of a single electron, $d w$, can then be estimated taking the limits of integration of Eq. 1.26 over an RF cycle:

$$
d w=\int_{-T / 2}^{T / 2} P d t=2 q \int_{0}^{T / 2} v_{d r i f t} E d t=2 q \int_{0}^{T / 2} \mu(E \sin \omega t)^{2} d t
$$

This also applies to the ions present in the cavity, meaning that an estimate of the energy loss per ion can also be made. The mobilities of hydrogen clusters $\left(\mathrm{H}_{3}^{+}\right.$, $\mathrm{H}_{5}^{+}$) and $\mathrm{O}_{2}^{-}$in hydrogen have been measured (Ref. [60-66] for hydrogen and [67] for oxygen). The drift velocity of $\mathrm{H}_{3}^{+}$and $\mathrm{H}_{5}^{+}$vs. $X$ and the mobility of $\mathrm{H}_{3}^{+}$vs. $P$ are shown in Fig. 1.22. The mobilities of ions used in this work are given in Tab.1.4 for reference. 


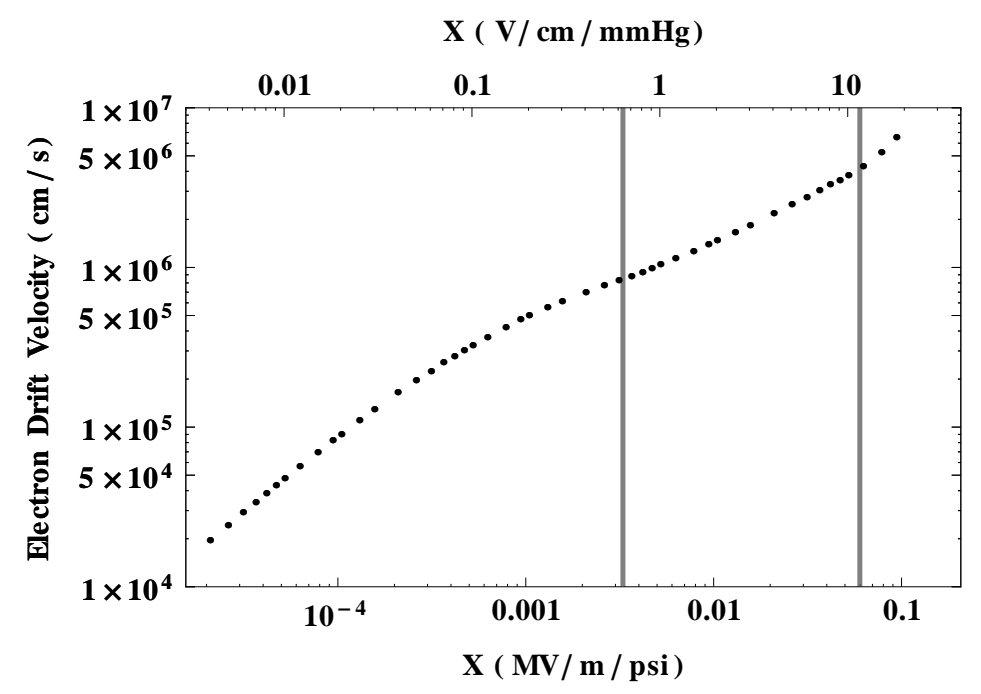

Figure 1.21. Electron drift velocity in hydrogen gas vs. $E / P[47]$. The vertical lines represent the range of $X$ in the HPRF beam test.
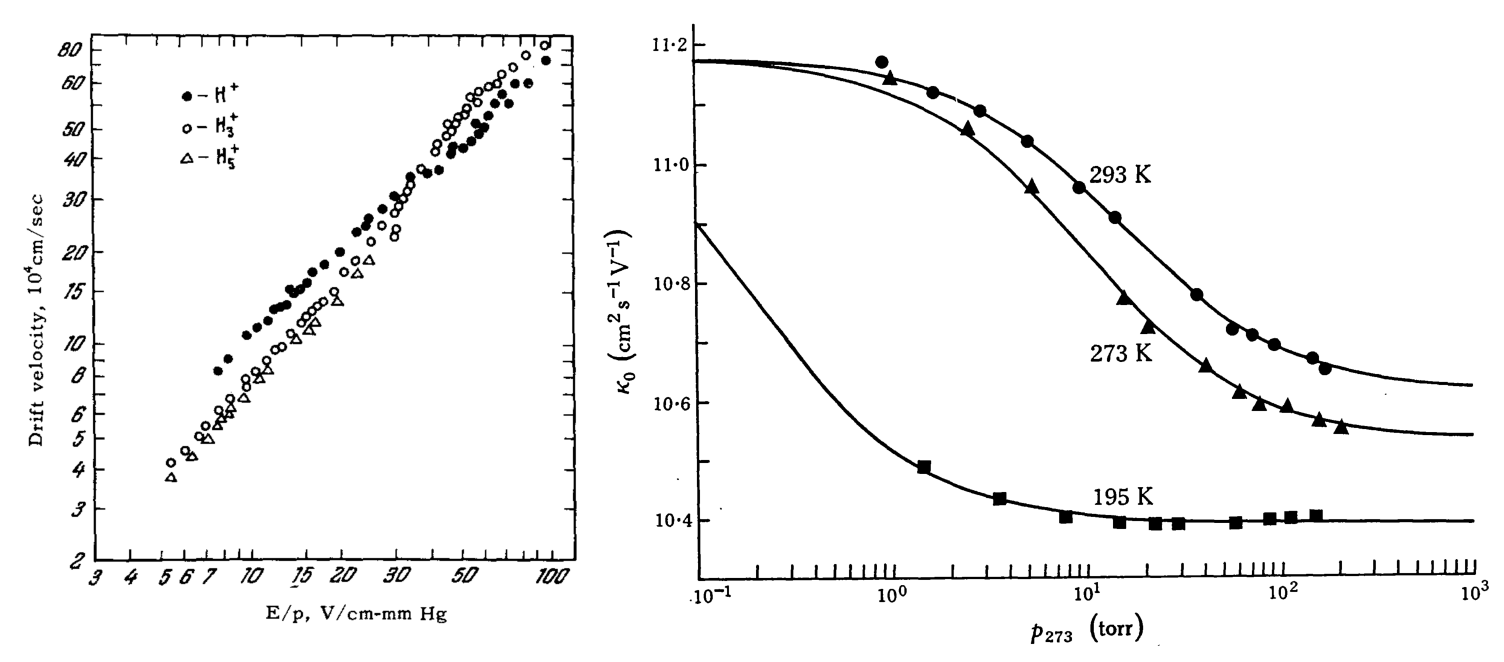

Figure 1.22. Drift velocities for $\mathrm{H}_{3}^{+}$and $\mathrm{H}_{5}^{+}$in hydrogen (left) [66]. Mobility of $\mathrm{H}_{3}^{+}$in hydrogen (right) [65]. 
Table 1.4. Ion mobilities in hydrogen.

\begin{tabular}{cc}
\hline \hline Ion & Reduced Mobility $\left(\frac{\mathrm{cm}^{2}}{\mathrm{Vs}}\right)$ \\
\hline $\mathrm{H}_{3}^{+}$ & 11.2 \\
$\mathrm{H}_{5}^{+}$ & 9.6 \\
$\mathrm{O}_{2}^{-}$ & 11.4 \\
\hline
\end{tabular}

1.2.4 Electron Kinetic Energy. The Einstein relation connects a particle's kinetic energy, mobility and diffusion rate in a gas:

$$
\frac{D}{\mu}=k_{B} T
$$

Measurements of the electron diffusion coefficient, $D$, and mobility, $\mu$, can be used to estimate the kinetic energy of an electron vs. $X$ [68-71]. Figure 1.23 shows the drift velocity and kinetic energy of an electron vs. $E / N(N$ is the number density of hydrogen) for gas temperatures of 77 and $300 \mathrm{~K}$. The data at $300 \mathrm{~K}$ will be used to estimate the kinetic energy of plasma electrons in the HPRF cavity later.

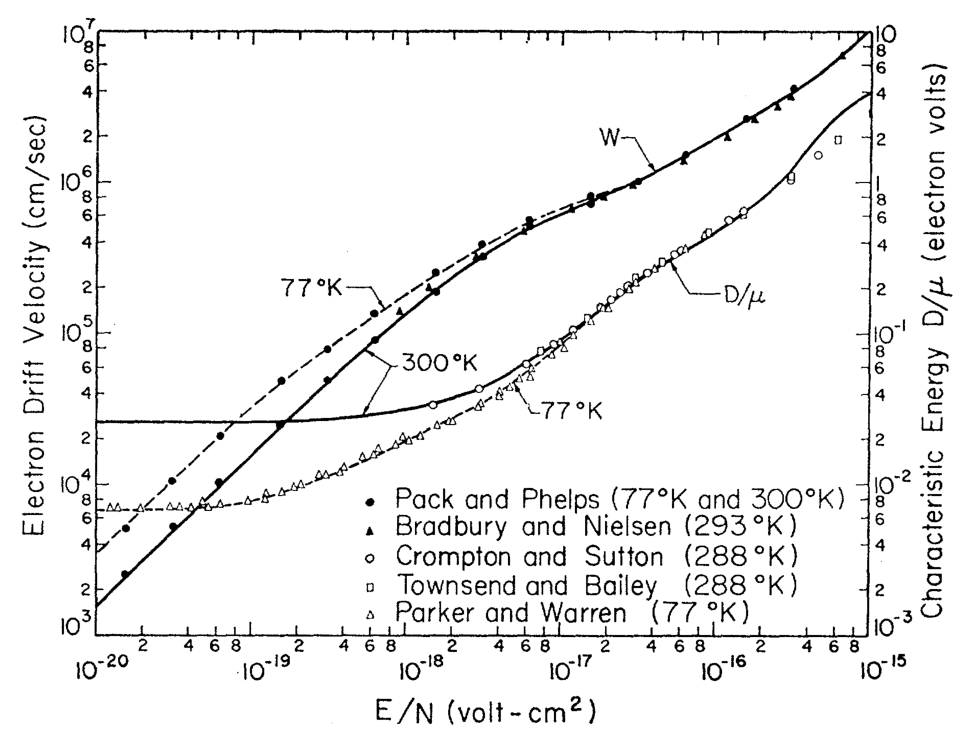

Figure 1.23. Electron drift velocity and kinetic energy in hydrogen vs. $E / N$ [69]. 
1.2.5 Recombination. If the HPRF cavity is filled with pure hydrogen, a plasma containing $e^{-}, \mathrm{H}_{3}^{+}, \mathrm{H}_{5}^{+}$, and potentially larger clusters of hydrogen form once the beam ionizes the hydrogen gas (and $\mathrm{H}_{2}^{+}$are converted to $\mathrm{H}_{3}^{+}$). Electrons may recombine with a positively charged ion. The recombination rate is given by the cross section, $\sigma$, and electron drift velocity, $v$ :

$$
\beta=\sigma v
$$

Rate equations for electrons and hydrogen ions are:

$$
\begin{aligned}
\frac{d n_{e}}{d t} & =\dot{n}_{e}-\sum_{m} \beta_{m} n_{e} n_{\mathrm{H}_{m}^{+}} \\
\frac{d n_{\mathrm{H}_{n}^{+}}}{d t} & =\dot{n}_{\mathrm{H}_{n}^{+}}-\sum_{m} \beta_{m} n_{e} n_{\mathrm{H}_{m}^{+}}
\end{aligned}
$$

where $n_{\alpha}$ is the number density of particle $\alpha, \dot{n}_{\alpha}$ is the production rate of particle $\alpha$, and $\beta_{m}$ is the recombination rate of electrons with $\mathrm{H}_{m}^{+}$. If we assume a hydrogen ion is produced for every electron produced and there is no other means of removing electrons or ions, then Eq. 1.32 reduces to:

$$
\frac{d n}{d t}=\dot{n}-\beta n^{2}
$$

The recombination rates for both $\mathrm{H}_{3}^{+}$and $\mathrm{H}_{5}^{+}$have been measured extensively using a variety of methods $\left(\mathrm{H}_{3}^{+}[72-76], \mathrm{H}_{5}^{+}[74-76]\right)$. It has been proposed that vibrationally excited ions recombine more slowly than ground state ions [72], for which there appears to be supporting evidence [74-76]. Figure 1.24 shows the recombination rates of $\mathrm{H}_{3}^{+}$and $\mathrm{H}_{5}^{+}$vs. electron temperature [76] in an where an $\mathrm{RF}$ cavity was used to heat the electrons while the gas temperature was kept constant. The desired species of ion was generated by fixing the concentrations of hydrogen and neon gases in the cavity. It can be seen that there is approximately an order of magnitude difference in the recombination rates of $\mathrm{H}_{3}^{+}$and $\mathrm{H}_{5}^{+}$. For $\mathrm{H}_{3}^{+}$there is almost a factor of two difference between these measurements and those made in [75]. 

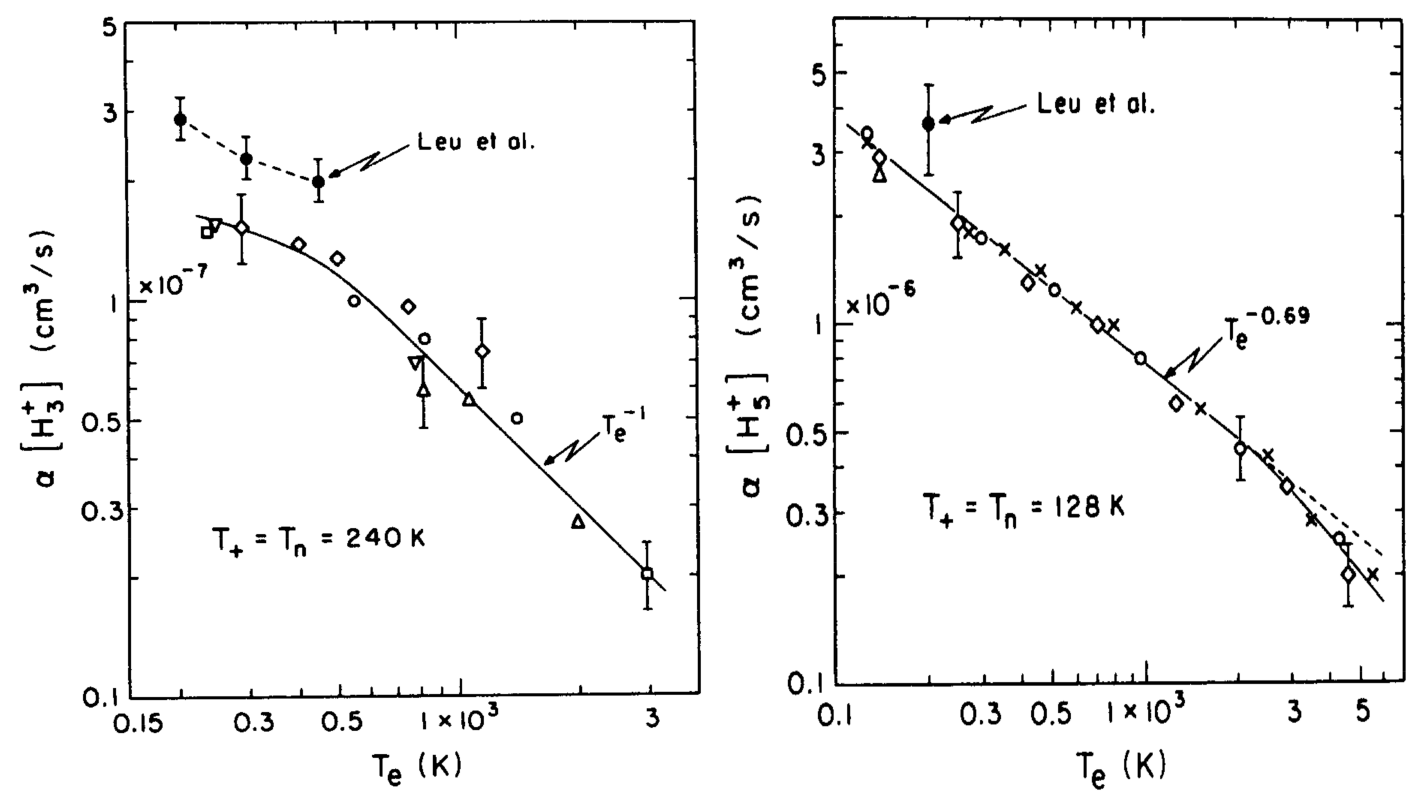

Figure 1.24. Recombination rate vs. electron temperature for $\mathrm{H}_{3}^{+}$(left) and $\mathrm{H}_{5}^{+}$ (right). The measurements were made at a gas temperature of $240 \mathrm{~K}$ for $\mathrm{H}_{3}^{+}$ and $128 \mathrm{~K}$ for $\mathrm{H}_{5}^{+}[76]$.

Figure 1.25 shows the results of a different technique [74]. Plotted is the effective recombination rate vs. hydrogen gas density. In that experiment, a plasma containing electrons and a mixture of $\mathrm{H}_{3}^{+}$and $\mathrm{H}_{5}^{+}$is created by adding argon and varying the hydrogen pressure and temperature to obtain the desired ratio of $\mathrm{H}_{3}^{+}$to $\mathrm{H}_{5}^{+}$. The electrons are allowed to come into thermal equilibrium with the gas, whose temperature is varied. The effective recombination rate is given by:

$$
\beta_{\text {eff }}=\left(\beta_{3}+\beta_{5} R\right) \frac{1}{1+R}, \quad R=\frac{n_{\mathrm{H}_{5}^{+}}}{n_{\mathrm{H}_{3}^{+}}}
$$

Note that as the temperature of the electrons increases, the effective recombination rate decreases. The plasma generated in our experiment will also contain a mixture of $\mathrm{H}_{3}^{+}$and $\mathrm{H}_{5}^{+}$. However, the hydrogen gas densities in our experiment are many orders of magnitude larger $\left(\sim 5-25 \times 10^{20} \mathrm{~cm}^{-3}\right.$ compared to $\left.6.5 \times 10^{17} \mathrm{~cm}^{-3}\right)$ than those of Ref. [76].

1.2.6 Attachment. When hydrogen is doped with an electronegative gas, in our 


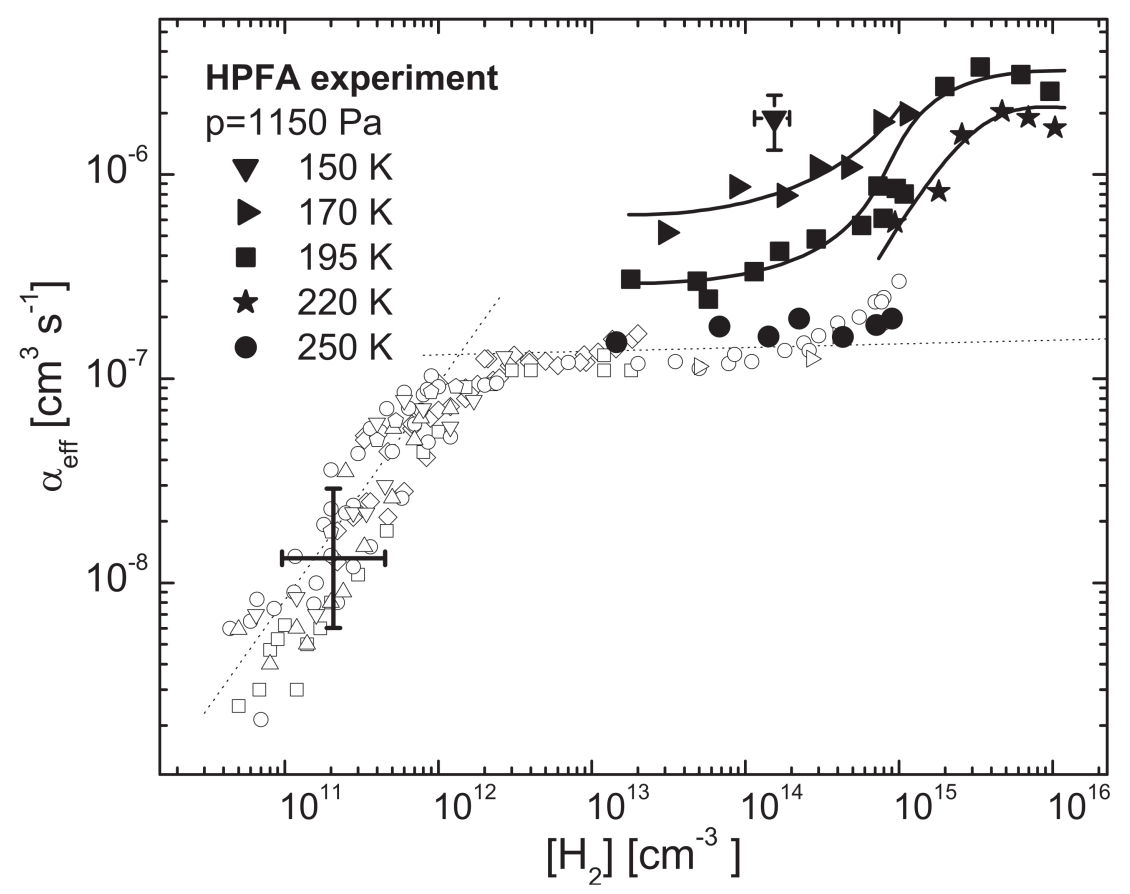

Figure 1.25. Effective recombination rate vs. hydrogen gas density at various gas temperatures [74]. The empty data points were taken at $260 \mathrm{~K}$.

case oxygen, a three-body attachment process takes place in the plasma. A description of the mechanism for attachment was first introduced by Bloch and Bradbury [77] and involves a two step process in which the first step is similar to recombination:

$$
e^{-}+\mathrm{O}_{2} \underset{t}{\stackrel{k_{a t}}{\rightleftharpoons}} \mathrm{O}_{2}^{-*}
$$

where $\mathrm{O}_{2}^{-*}$ is an excited state of oxygen, $k_{a t}$ is the attachment coefficient of $\mathrm{O}_{2}^{-*}$ formation, and $t$ is the lifetime of $\mathrm{O}_{2}^{-*}$ before it decays into the initial particles. An estimation of the collision frequency will give an idea of how frequently the excited state reverts back to the original conditions. The collision frequency is given by:

$$
\nu=v \sigma n
$$

where $v$ is the ion drift velocity, $\sigma$ the cross section of oxygen, and $n$ the gas number density. Assuming the cross section is constant at around $7.3 \times 10^{-16} \mathrm{~cm}^{2}$ (based on the Van der Waals radius of oxygen, $150 \mathrm{pm}$ ), the collision rate ranges from 5.8 
to $29 \times 10^{10} \mathrm{~s}^{-1}$. This gives an effective time between collisions of $3.5-17 \mathrm{ps}$. The lifetime $t$ has been calculated to be $0.1-1 \mathrm{~ns}$, and so we will assume very few electrons are lost after being captured by oxygen [78].

One of two things can take place at this point. Either the oxygen can be deexcited by a collision with another gas molecule $(M)$, or a collision with said molecule can ionize the oxygen molecule:

$$
\begin{aligned}
& \mathrm{O}_{2}^{-*}+M \stackrel{k_{T}}{\longrightarrow} \mathrm{O}_{2}^{-}+M \\
& \underset{k_{I}}{\longrightarrow} e^{-}+\mathrm{O}_{2}+M
\end{aligned}
$$

Here, $k_{T}$ is the attachment coefficient of de-excitation, and $k_{I}$ is the attachment coefficient of ionization. The attachment coefficient for the three-body process (Eq. 1.35 and Eq. 1.37) depends on $k_{a t}$ multiplied by the probability that $\mathrm{O}_{2}^{-*}$ will de-excite:

$$
k_{e f f}=\frac{k_{a t} k_{T}}{t^{-1}+\left(k_{T}+k_{I}\right) n_{M}}
$$

where $n_{M}$ is the density of the third body. Figure 1.26 shows the potential energy curve for $\mathrm{O}_{2}$ and $\mathrm{O}_{2}^{-}$. The ground state energy of $\mathrm{O}_{2}^{-}$is $\approx 0.43 \mathrm{eV}$ lower than that of $\mathrm{O}_{2}$. It is possible that $\mathrm{O}_{2}^{-*}$ will dissociate in a collision with another particle, but the required energy is $\approx 4 \mathrm{eV}$. Only the tail of the energy distribution of electrons in the cavity can approach this value (the maximum average kinetic energy is $1 \mathrm{eV}$ for our range of $X$, from Fig. 1.23), so dissociation is very unlikely.

The rate equation becomes:

$$
\frac{d n_{e}}{d t}=\dot{n}_{e}-\sum \beta_{l} n_{e} n_{H_{l}^{+}}-\sum k_{m} n_{e} n_{\mathrm{O}_{2}} n_{m}
$$

where the sum over $l$ is for each cluster of hydrogen, and the sum over $m$ is for each species of gas molecule. For almost all cases, $\sum \beta_{l} n_{H_{l}^{+}} \ll \sum k_{m} n_{\mathrm{O}_{2}} n_{m}$.

Unfortunately there are no measurements in the literature of the attachment coefficient of electrons in a hydrogen-oxygen-nitrogen mixture, like we have for the 


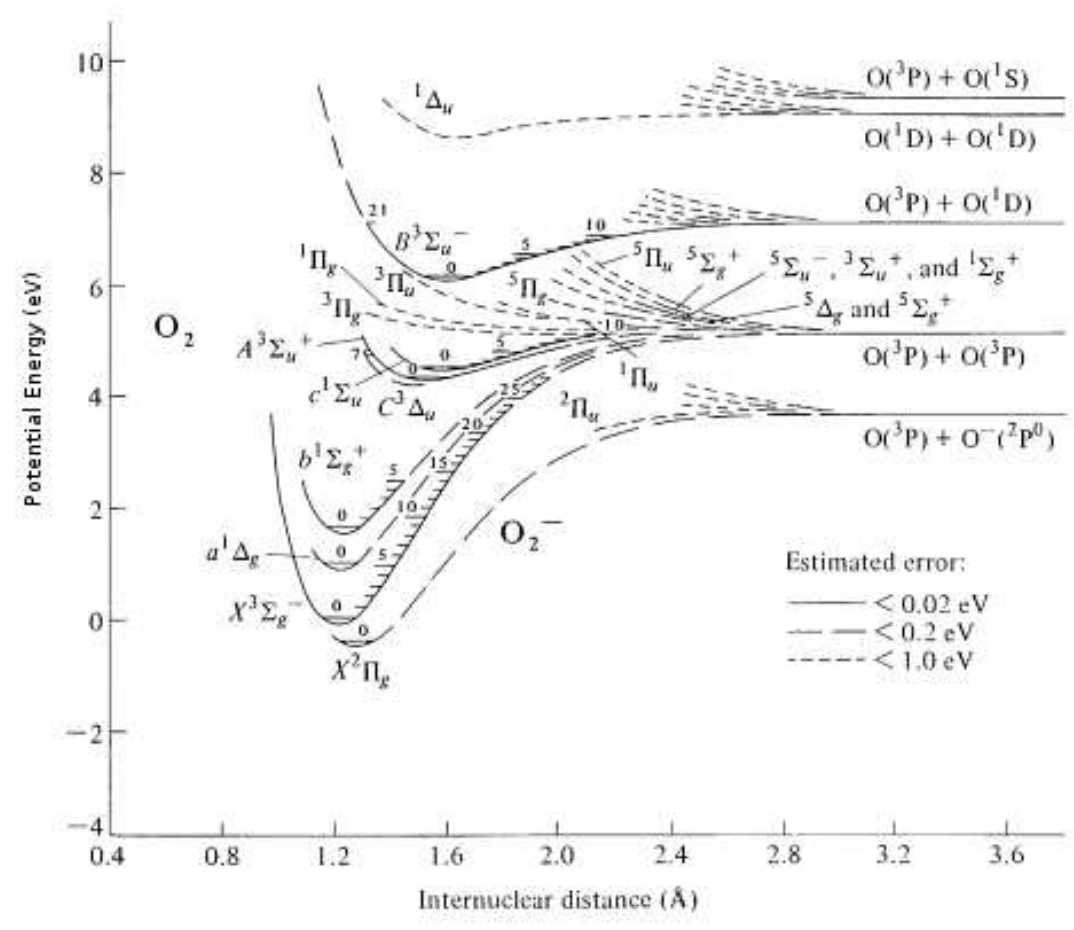

Figure 1.26. Potential energy curves for $\mathrm{O}_{2}$ and $\mathrm{O}_{2}^{-}$[79].

case of dry air-doped hydrogen. Most measurements have been made in pure oxygen $[78,80-88]$, or in oxygen-nitrogen mixtures $[78,80-82,84-86]$. Only a few sources report the attachment coefficient in an oxygen-hydrogen mixture [80,89].

Figure 1.27 shows the attachment rate $\left(k_{a t}\right)$ of electrons by oxygen in hydrogen vs. hydrogen density. The slope of the fit to the data gives the attachment coefficient, which is reported to be $4.8 \pm 0.3 \times 10^{-31} \mathrm{~cm}^{6} / \mathrm{s}$ [89]. The attachment coefficient given in [80] is $2.0 \times 10^{-31} \mathrm{~cm}^{6} / \mathrm{s}$. Unfortunately no data on the attachment coefficient as functions of gas pressure or electron energy exist.

Figure 1.28 shows the attachment coefficients for oxygen and helium vs. average electron energy for different pressures.

Figure 1.29 shows the attachment coefficients and attachment rates for nitrogen vs. average electron energy. Note that as the nitrogen pressure increases, so does the attachment rate. 


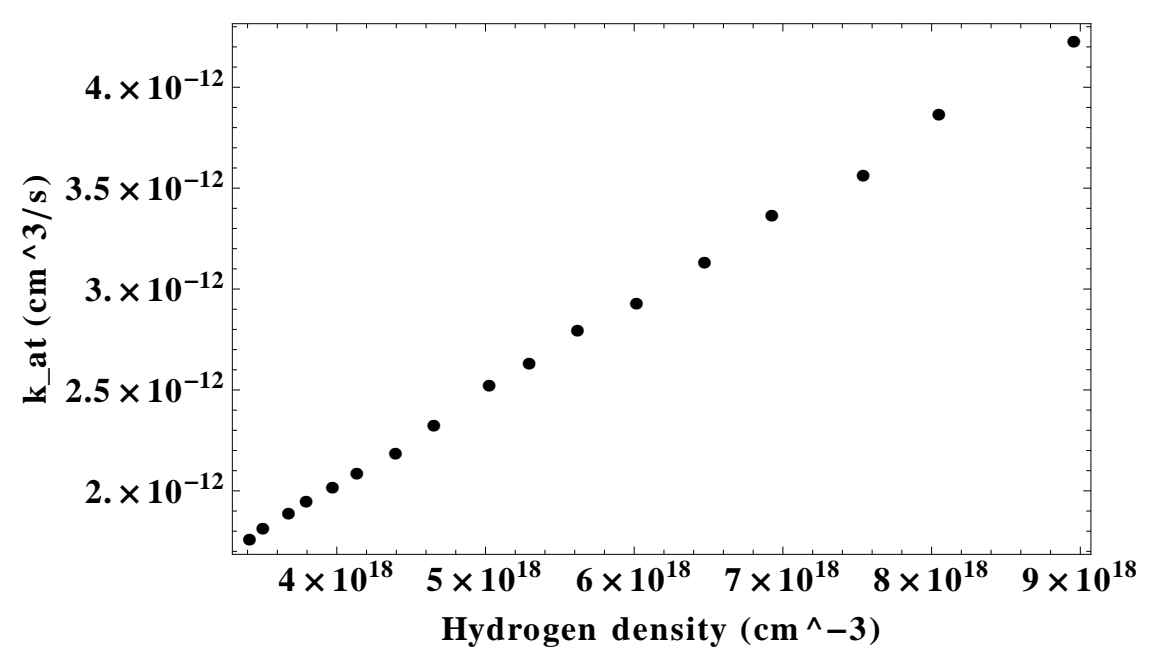

Figure 1.27. Attachment rate vs. hydrogen density [89]. The points are measurements. The slope of the line fitted to the data gives the attachment coefficient.

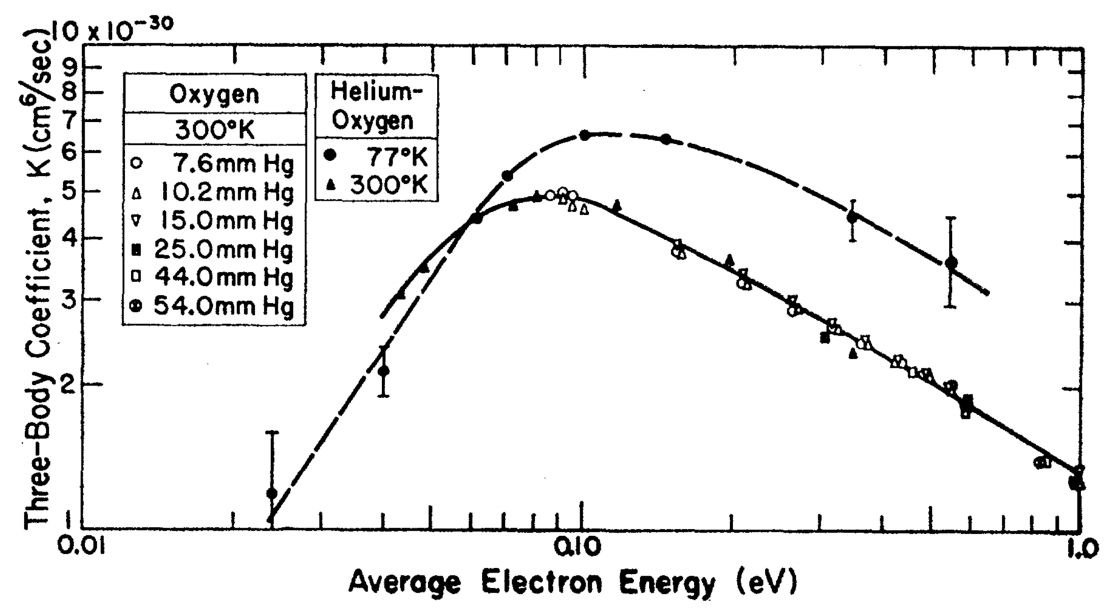

Figure 1.28. Attachment coefficients for oxygen and helium vs. electron energy [81]. The oxygen data are taken at $300 \mathrm{~K}$ at various pressures. 

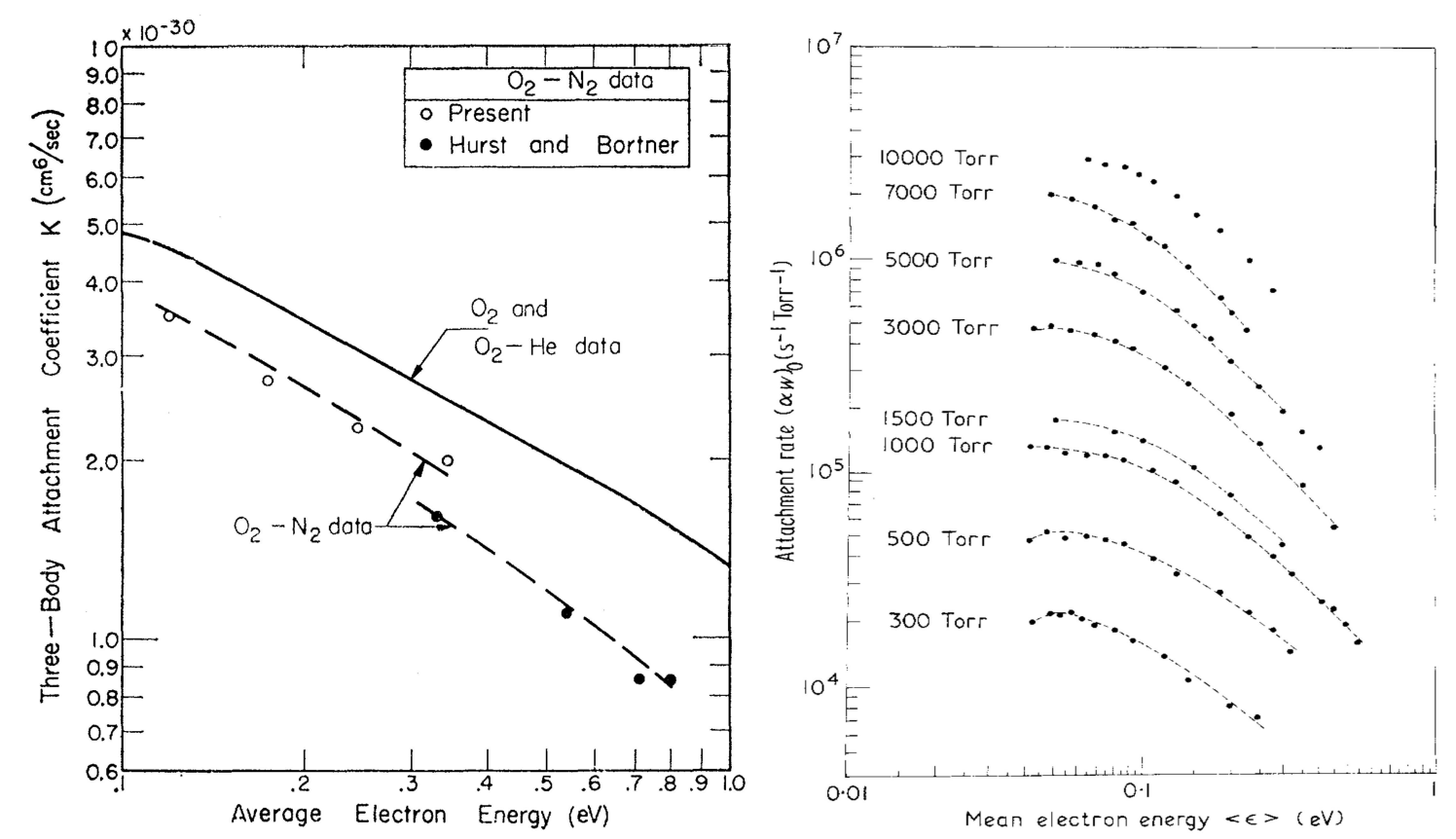

Figure 1.29. Attachment coefficient (left) [81] and attachment rate (right) [86] of nitrogen vs. average electron energy.

Figure 1.30 shows the attachment coefficient vs. oxygen concentration in helium for various $X$ at $300 \mathrm{~K}$ and the attachment rate vs. oxygen partial pressure at 300 torr for various average electron energies. The slopes of the fits in the right plot give the rates for oxygen as the third body, while the intercepts give the rates for helium or nitrogen as the third body.

Kossyi gives equations for the attachment coefficients for oxygen and nitrogen as functions of gas and electron temperature (in K) [82]:

$$
\begin{aligned}
& k_{\mathrm{O}_{2}}=1.4 \times 10^{-29} \frac{300}{T_{e}} e^{-\frac{600}{T}} e^{\frac{700\left(T_{e}-T\right)}{T_{e} T}}\left[\frac{\mathrm{cm}^{6}}{\mathrm{~s}}\right] \\
& k_{N_{2}}=1.07 \times 10^{-31}\left(\frac{300}{T_{e}}\right)^{2} e^{-\frac{70}{T}} e^{\frac{1500\left(T_{e}-T\right)}{T_{e} T}}\left[\frac{\mathrm{cm}^{6}}{\mathrm{~s}}\right]
\end{aligned}
$$

Table 1.5 shows selected three-body attachment coefficients for thermal electrons in various third-body gases at $300 \mathrm{~K}$. Note that the numbers given are all for total gas pressures much lower than those in the HPRF experiment. The relative rate 

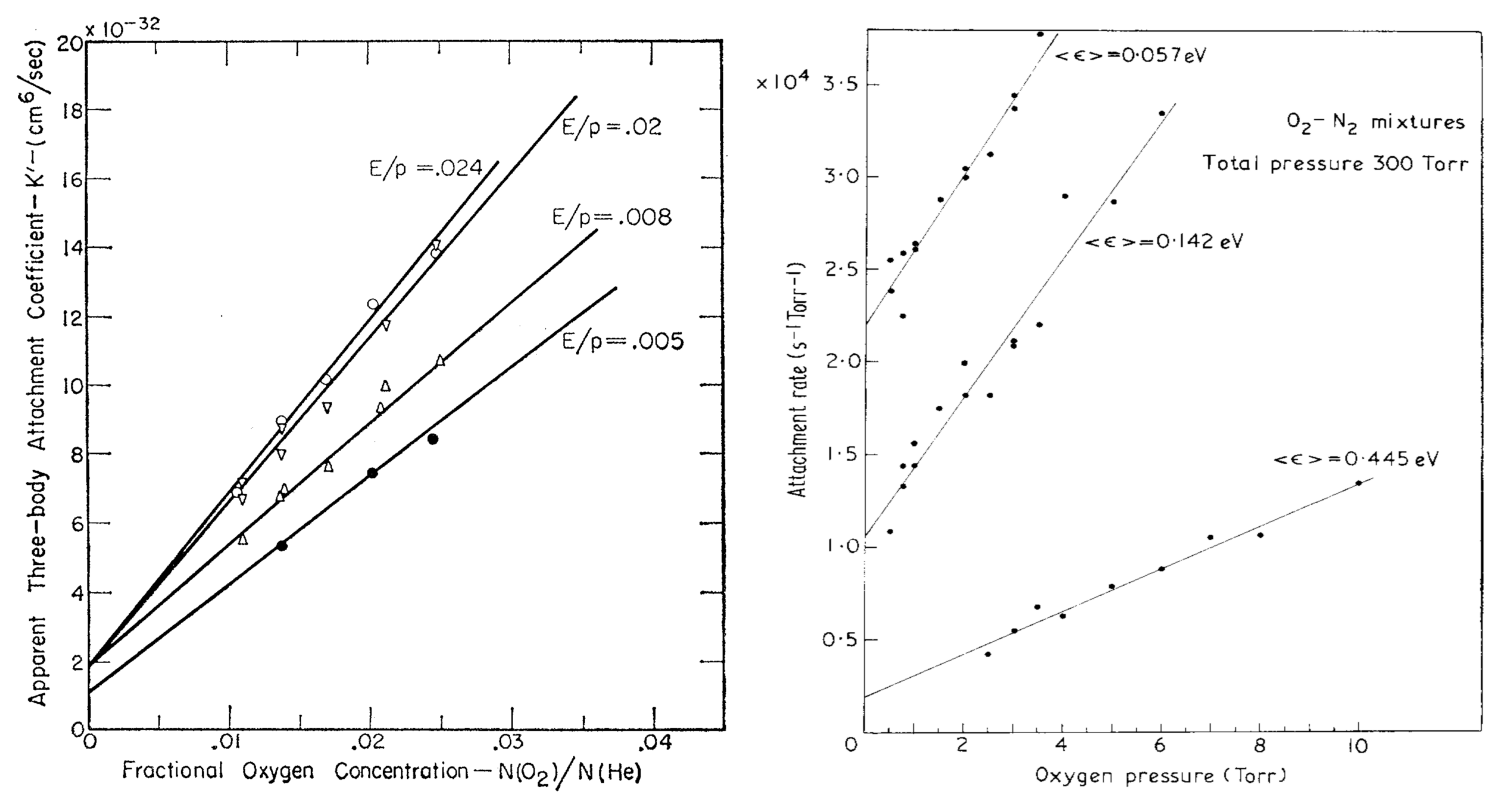

Figure 1.30. Attachment coefficient (left) vs. oxygen concentration in helium at various values of $X$ at $300 \mathrm{~K}$ [81]. Attachment rate (right) vs. oxygen pressure in nitrogen at 300 torr for various average electron energies [86]

of hydrogen as a third body is $2-3$ times that of nitrogen, and $1 / 10-1 / 5$ that of oxygen.

Table 1.5. Various three-body attachment coefficient measurements of electrons to oxygen in various gases at $300 \mathrm{~K}$.

\begin{tabular}{lc}
\hline \hline Gas & Attachment coefficient $\left(10^{-31} \frac{\mathrm{cm}^{6}}{\mathrm{~s}}\right)$ \\
\hline $\mathrm{H}_{2}$ & $2.0,4.8$ \\
$\mathrm{O}_{2}$ & $20,21.2,25,28$ \\
$\mathrm{~N}_{2}$ & $1.0,1.1,1.6$ \\
\hline
\end{tabular}


1.2.7 Ion-Ion Recombination. Ions can recombine, or become neutral, through two processes. The first is through binary collisions of the form [80]:

$$
A^{-}+B^{+} \rightarrow A+B^{*}
$$

where the energy released in the reaction goes into exciting molecule $B$. This process dominates below 30 torr (0.58 psi) [80], and so we will ignore it. The dominant process in our case is a three-body reaction similar to that of electron attachment:

$$
A^{-}+B^{+}+M \rightarrow A+B+M
$$

For pressures above 1 atm (14.7 psi), recombination can be described by the Langevin model [90]. In this regime, the drift velocity of the positive and negative ions with respect to each other must be large enough to overcome the energy they lose through collisions with neutral molecules. The ions drift in a Coulomb field with relative drift velocity:

$$
v_{d r i f t}=\frac{e}{\left(4 \pi \epsilon_{0}\right) r^{2}}\left(\mu_{+}+\mu_{-}\right)
$$

where $\mu$ is the mobility. The recombination rate is given by [90]:

$$
k_{i i}=4 \pi r^{2} v_{d r i f t}=\frac{e}{\epsilon_{0}}\left(\mu_{+}+\mu_{-}\right)
$$

Note that this is not explicitly dependent on gas pressure. However, the mobility of the ions is, and is believed to reach a peak around $1 \mathrm{~atm}$, and then fall with increasing pressure. Figure 1.31 shows a general curve of recombination rate vs. pressure. The curve peaks at around $2 \times 10^{-6} \frac{\mathrm{cm}^{3}}{\mathrm{~s}}$ and then falls steadily with pressure. Using the mobilities of $\mathrm{H}_{5}^{+}$and $\mathrm{O}_{2}^{-}$in Tab. 1.4 and Eq. 1.45 , one gets a rate of $3.8 \times 10^{-5} \frac{\mathrm{cm}^{3}}{\mathrm{~s}}$.

Unfortunately, no data on the ion-ion recombination rates of $\mathrm{H}_{5}^{+}$or $\mathrm{H}_{3}^{+}$with $\mathrm{O}_{2}^{-}$could be found. A variety of other ion rates are available, and are listed in Tab. 1.6 [90-99]. 


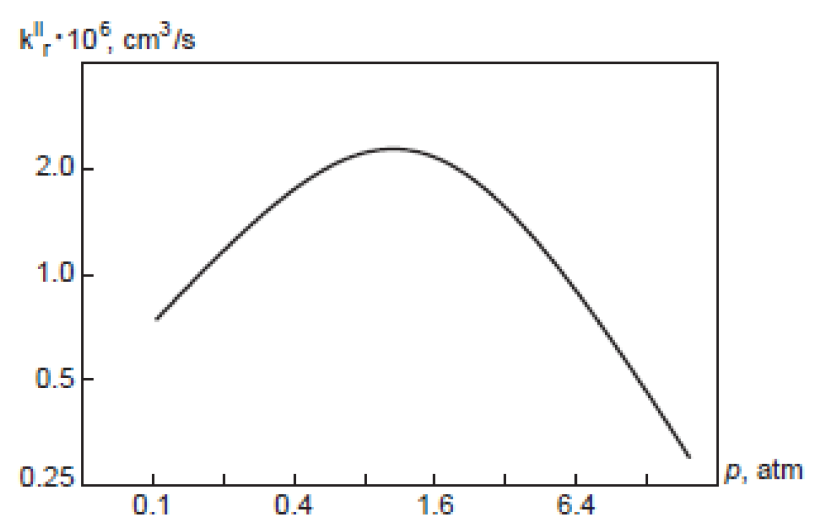

Figure 1.31. Ion-ion recombination rate vs. gas pressure.

Table 1.6. Ion-ion recombination rates.

\begin{tabular}{lll}
\hline \hline Reaction & Rate $\left(10^{-6} \frac{\mathrm{cm}^{3}}{\mathrm{~s}}\right)$ & Gas density, temperature \\
& & or pressure \\
$\mathrm{O}_{2}^{-}+\mathrm{O}_{4}^{+}+\mathrm{O}_{2} \rightarrow 4 \mathrm{O}_{2}$ & 4.2 & $2.7 \times 10^{19} \mathrm{~cm}^{-3}$ \\
$\mathrm{O}_{2}^{-}+\mathrm{O}_{4}^{+}+\mathrm{O}_{2} \rightarrow \mathrm{O}_{6}+\mathrm{O}_{2}$ & 2.2 & $2.7 \times 10^{19} \mathrm{~cm}^{-3}$ \\
$\mathrm{O}_{2}^{-}+\mathrm{O}_{4}^{+}+\mathrm{O}_{2} \rightarrow \mathrm{O}_{6}+\mathrm{O}_{2}$ & 0.3 & $5.4 \times 10^{20} \mathrm{~cm}^{-3}$ \\
$\mathrm{O}_{2}^{-}+\mathrm{O}_{2}^{+} \rightarrow \mathrm{O}_{2}+\mathrm{O}_{2}^{*}$ & 0.14 & $200 \mathrm{~K}$ \\
$\mathrm{O}_{2}^{-}+\mathrm{O}_{2}^{+} \rightarrow \mathrm{O}_{2}+\mathrm{O}_{2}^{*}$ & 0.0892 & $500 \mathrm{~K}$ \\
$N_{2}^{-}+\mathrm{NO}^{+}+\mathrm{H}_{2} \rightarrow$ & 0.76 & $2 \times 10^{19} \mathrm{~cm}^{-3}$ \\
$F^{-}+\mathrm{Kr}^{+}+\mathrm{Ar} \rightarrow$ & 0.2 & $80 \mathrm{~atm}$ \\
$H^{-}+\mathrm{H}^{+} \rightarrow$ & 0.039 & Thermal \\
$\mathrm{O}_{2}^{-}+N_{2}^{+} \rightarrow$ & 0.16 & Thermal \\
$H^{-}+\mathrm{H}^{+} \rightarrow$ & 0.40 &
\end{tabular}


1.2.8 Radio Frequency Cavity Operation. At the MuCool Test Area (MTA), $\mathrm{RF}$ power is fed from a klystron into the HPRF cavity through a series of rectangular and coaxial waveguides.

1.2.8.1 Waveguides. This section and the next closely follow Chapter 8 of Ref. [100]. For the case of a cylindrical waveguide, a time dependence for the electromagnetic fields of the form $e^{-i \omega t}$ gives Maxwell's equations in the form:

$$
\begin{array}{ll}
\nabla \times \mathbf{E}=i \omega \mathbf{B} & \nabla \cdot \mathbf{E}=0 \\
\nabla \times \mathbf{B}=-i \mu \epsilon \omega \mathbf{E} & \nabla \cdot \mathbf{B}=0
\end{array}
$$

where the waveguide is filled with medium of permittivity $\epsilon$ and permeability $\mu$. Both $\mathbf{E}$ and $\mathbf{B}$ satisfy the wave equation:

$$
\left(\nabla^{2}+\mu \epsilon \omega^{2}\right)\left\{\begin{array}{l}
\mathbf{E} \\
\mathbf{B}
\end{array}\right\}=0
$$

Assuming the $z$ direction is along the axis of the cylinder, the fields can be separated into:

$$
\left.\begin{array}{l}
\mathbf{E}(x, y, z, t) \\
\mathbf{B}(x, y, z, t)
\end{array}\right\}=\left\{\begin{array}{l}
\mathbf{E}(x, y) e^{ \pm i k z-i \omega t} \\
\mathbf{B}(x, y) e^{ \pm i k z-i \omega t}
\end{array}\right.
$$

The wave equation reduces to the two dimensional form:

$$
\left[\nabla_{t}^{2}+\left(\mu \epsilon \omega^{2}-k^{2}\right)\right]\left\{\begin{array}{l}
\mathbf{E} \\
\mathbf{B}
\end{array}\right\}=0
$$

where $k$ is the wave number and $\nabla_{t}^{2}$ is the transverse part of the Laplacian operator:

$$
\nabla_{t}^{2}=\nabla^{2}-\frac{\partial^{2}}{\partial z^{2}}
$$

Separating the fields into their parallel and transverse components:

$$
\begin{aligned}
& \mathbf{E}=\mathbf{E}_{z}+\mathbf{E}_{t} \\
& \mathbf{E}_{z}=E_{z} \hat{\mathbf{z}} \\
& \mathbf{E}_{t}=(\hat{\mathbf{z}} \times \mathbf{E}) \times \hat{\mathbf{z}}
\end{aligned}
$$


Maxwell's equations can be written in terms of longitudinal and transverse components:

$$
\begin{array}{ll}
\frac{\partial \mathbf{E}_{t}}{\partial z}+i \omega \hat{\mathbf{z}} \times \mathbf{B}_{t}=\nabla_{t} E_{z} & \hat{\mathbf{z}} \cdot\left(\nabla_{t} \times \mathbf{E}_{t}\right)=i \omega B_{z} \\
\frac{\partial \mathbf{B}_{t}}{\partial z}+i \mu \epsilon \omega \hat{\mathbf{z}} \times \mathbf{E}_{t}=\nabla_{t} B_{z} & \hat{\mathbf{z}} \cdot\left(\nabla_{t} \times \mathbf{E}_{t}\right)=-i \mu \epsilon \omega E_{z} \\
\nabla_{t} \cdot \mathbf{E}_{t}=-\frac{\partial E_{z}}{\partial z} & \nabla_{t} \cdot \mathbf{B}_{t}=-\frac{\partial B_{z}}{\partial z}
\end{array}
$$

Solutions to Eq. 1.52 that have only field components in the transverse direction are called transverse electromagnetic (TEM) waves. Setting $E_{z}=0$ and $B_{z}=0$, with $\mathbf{E}_{t}=\mathbf{E}_{T E M}$, implies:

$$
\begin{aligned}
& \nabla_{t} \times \mathbf{E}_{T E M}=0 \\
& \nabla_{t} \cdot \mathbf{E}_{T E M}=0
\end{aligned}
$$

This means the axial wave number is the infinite medium value:

$$
k=k_{0}=\omega \sqrt{\mu \epsilon}
$$

The magnetic field is related to the electric by:

$$
\mathbf{B}_{T E M}= \pm \sqrt{\mu \epsilon} \hat{\mathbf{z}} \times \mathbf{E}_{T E M}
$$

The boundary conditions for a perfectly conducting cylinder are:

$$
\begin{aligned}
\left.E_{z}\right|_{S} & =0 \\
\left.\frac{\partial B_{z}}{\partial n}\right|_{S} & =0
\end{aligned}
$$

where $\partial / \partial n$ is the normal derivative at a point on the surface. The TEM mode cannot exist inside a cylindrical conductor with infinite conductivity; the electric field vanishes inside the surface. There are two distinct modes, defined by $B_{z}=0$ everywhere, with the boundary condition $\left.E_{z}\right|_{S}=0$ (called Transverse Magnetic (TM) waves); and $E_{z}=0$ everywhere (called Transverse Electric (TE) waves). 
For wave propagation inside a hollow waveguide of uniform cross section, the transverse electric and magnetic fields are related by:

$$
\mathbf{H}_{t}=\frac{ \pm 1}{Z} \hat{\mathbf{z}} \times \mathbf{E}_{t}
$$

where the wave impedance, $Z$, is given by:

$$
Z= \begin{cases}\frac{k}{\epsilon \omega}=\frac{k}{k_{0}} \sqrt{\frac{\mu}{\epsilon}} & (\mathrm{TM}) \\ \frac{\mu \omega}{k}=\frac{k_{0}}{k} \sqrt{\frac{\mu}{\epsilon}} & (\mathrm{TE})\end{cases}
$$

Making the substitution for $\mathrm{TM}(\mathrm{TE})$ waves, $E_{z}\left(H_{z}\right)=\psi(x, y) e^{ \pm i k z}$, one gets the transverse fields as functions of the longitudinal fields:

$$
\begin{aligned}
& \mathbf{E}_{t}= \pm \frac{i k}{\gamma^{2}} \nabla_{t} \psi \quad \text { TM Waves } \\
& \mathbf{H}_{t}= \pm \frac{i k}{\gamma^{2}} \nabla_{t} \psi \quad \text { TE Waves }
\end{aligned}
$$

where:

$$
\gamma^{2}=\mu \epsilon \omega^{2}-k^{2}
$$

and $\psi$ satisfies the two dimensional Laplacian equation $\left(\nabla_{t}^{2}+\gamma^{2}\right) \psi=0$ with boundary conditions $\left.\psi\right|_{S}=0$ or $\partial \psi /\left.\partial n\right|_{S}=0$ for TM or TE waves. This specifies an eigenvalue problem with eigenvalues $\gamma_{\lambda}^{2}$ and solutions $\psi_{\lambda}$ for $\lambda=1,2,3, \ldots$ The solutions are called the modes of the waveguide. The wave number for a given solution is:

$$
k_{\lambda}^{2}=\mu \epsilon \omega^{2}-\gamma_{\lambda}^{2}
$$

The cutoff frequency is defined as:

$$
\omega_{\lambda}=\frac{\gamma_{\lambda}}{\sqrt{\mu \epsilon}}
$$

and the wavenumber can be rewritten:

$$
k_{\lambda}=\sqrt{\mu \epsilon} \sqrt{\omega^{2}-\omega_{\lambda}^{2}}
$$


When $\omega<\omega_{\lambda}, k_{\lambda}$ is imaginary and these modes cannot propagate in the waveguide.

The modes of a rectangular waveguide filled with a material with permeability $\mu$ and permittivity $\epsilon$ and inner dimensions $a$ and $b$ can be found. Consider the case of the TE modes. The wave equation for $\psi=H_{z}$ is:

$$
\left(\frac{\partial^{2}}{\partial x^{2}}+\frac{\partial^{2}}{\partial y^{2}}+\gamma^{2}\right) \psi=0
$$

With boundary conditions $\partial \psi / \partial n=0$ at $x=0, a$ and $y=0, b$, the solution is:

$$
\psi_{m n}(x, y)=H_{0} \cos \left(\frac{\pi x}{a}\right) \cos \left(\frac{n \pi y}{b}\right)
$$

where:

$$
\gamma_{m n}^{2}=\pi^{2}\left(\frac{m^{2}}{a^{2}}+\frac{n^{2}}{b^{2}}\right)
$$

Here, $\gamma$ has been replaced by integers $m, n$, which cannot both be zero for nontrivial solutions. The cutoff frequency is:

$$
\omega_{m n}=\frac{\pi}{\sqrt{\mu \epsilon}}\left(\frac{m^{2}}{a^{2}}+\frac{n^{2}}{b^{2}}\right)^{1 / 2}
$$

The lowest frequency TE mode for $a>b$ has the cutoff frequency:

$$
\omega_{1,0}=\frac{\pi}{\sqrt{\mu \epsilon} a}
$$

The fields for this $\mathrm{TE}_{1,0}$ mode are:

$$
\begin{aligned}
& H_{z}=H_{0} \cos \left(\frac{\pi x}{a}\right) e^{i k z-i \omega t} \\
& H_{x}=-\frac{i k a}{\pi} H_{0} \sin \left(\frac{\pi x}{a}\right) e^{i k z-i \omega t} \\
& E_{y}=i \frac{\omega a \mu}{\pi} H_{0} \sin \left(\frac{\pi x}{a}\right) e^{i k z-i \omega t}
\end{aligned}
$$

For TM modes, $E_{z}$ is:

$$
E_{z}=E_{0} \sin \left(\frac{m \pi x}{a}\right) \sin \left(\frac{n \pi y}{b}\right)
$$

The lowest mode in Eq. 1.71 is $m=n=1$. 
1.2.8.2 Resonant Cavities. Closing the ends of a cylindrical waveguide can create standing waves. Assuming the walls have infinite conductivity and the cavity is filled with a material with properties $\mu$ and $\epsilon$, if the boundaries are at $z=0$ and $z=d$, then the boundary conditions are:

$$
k=p \frac{\pi}{d}, \quad p=0,1,2, \ldots
$$

For TM fields:

$$
\begin{aligned}
& E_{z}=\psi(x, y) \cos \left(\frac{p \pi z}{d}\right) \\
& \mathbf{E}_{\mathbf{t}}=-\frac{p \pi}{d \gamma^{2}} \sin \left(\frac{p \pi z}{d}\right) \nabla_{t} \psi \\
& \mathbf{H}_{\mathbf{t}}=\frac{i \epsilon \omega}{\gamma^{2}} \cos \left(\frac{p \pi z}{d}\right) \hat{\mathbf{z}} \times \nabla_{t} \psi
\end{aligned}
$$

and for TE fields:

$$
\begin{aligned}
& H_{z}=\psi(x, y) \sin \left(\frac{p \pi z}{d}\right) \\
& \mathbf{E}_{\mathbf{t}}=-\frac{i \omega \mu}{\gamma^{2}} \sin \left(\frac{p \pi z}{d}\right) \hat{\mathbf{z}} \times \nabla_{t} \psi \\
& \mathbf{H}_{\mathbf{t}}=\frac{p \pi}{d \gamma^{2}} \cos \left(\frac{p \pi z}{d}\right) \nabla_{t} \psi
\end{aligned}
$$

where:

$$
\gamma^{2}=\mu \epsilon \omega^{2}-\left(\frac{p \pi}{d}\right)^{2}
$$

The eigenfrequency for a given value of $p$, determined by the eigenvalue $\gamma^{2}$, is:

$$
\omega_{p}^{2}=\frac{1}{\mu \epsilon}\left[\gamma^{2}+\left(\frac{p \pi}{d}\right)^{2}\right]
$$

For a cylindrical cavity with radius $R$, the TM mode for $\psi=E z$ with the boundary condition $E_{z}=0$ at $\rho=R$, has the solution:

$$
\psi(\rho, \phi)=E_{0} J_{m}\left(\gamma_{m n} \rho\right) e^{ \pm i m \phi}
$$

where $J_{m}$ are Bessel functions,

$$
\gamma_{m n}=\frac{x_{m n}}{R}
$$


and $x_{m n}$ is the $n$th root of $J_{m}(x)$. The resonant frequencies are given by:

$$
\omega_{m n p}=\frac{1}{\sqrt{\mu \epsilon}} \sqrt{\operatorname{fracx}_{m n}^{2} R^{2}+\frac{p^{2} \pi^{2}}{d^{2}}}
$$

$\mathrm{TM}_{0,1,0}$ is the lowest mode and has a resonant frequency of:

$$
\omega_{010}=\frac{2.405}{\sqrt{\mu \epsilon} R}
$$

The explicit expressions for the electric and magnetic fields are then:

$$
\begin{aligned}
& E_{z}=E_{0} J_{0}\left(\frac{2.405 \rho}{R}\right) e^{-i \omega t} \\
& H_{\phi}=-i \sqrt{\frac{\epsilon}{\mu}} E_{0} J_{1}\left(\frac{2.405 \rho}{R}\right) e^{-i \omega t}
\end{aligned}
$$

The lowest $\mathrm{TE}$ mode is $\mathrm{TE}_{1,1,1}$ and has a resonant frequency:

$$
\omega_{111}=\frac{1.841}{\sqrt{\mu \epsilon} R}\left(1+2.912 \frac{R^{2}}{d^{2}}\right)^{1 / 2}
$$

The longitudinal component of the magnetic field is:

$$
H_{z}=H_{0} J_{1}\left(\frac{1.841 \rho}{R}\right) \cos \phi \sin \left(\frac{\pi z}{d}\right) e^{-i \omega t}
$$

For $d>2.03 R$ the resonant frequency $\omega_{111}$ is smaller than that of the lowest TM mode, making the $\mathrm{TE}_{1,1,1}$ the lowest frequency mode of the cavity. Therefore the ratio of $d$ to $R$ is important in determining the fundamental mode of the cavity.

The quality factor, $Q$ of a cavity is:

$$
Q=\omega_{0} \frac{\text { average energy stored }}{\text { energy loss } / \text { second }}
$$

The time rate of change of the stored energy in the cavity is:

$$
\frac{d U}{d t}=-\frac{\omega_{0}}{Q} U
$$

whose solution is:

$$
U(t)=U_{0} e^{-\omega_{0} t / Q}
$$


The energy stored in a cavity with mode $\lambda, p$ is:

$$
\begin{aligned}
& U=\frac{d}{4} \epsilon\left[1+\left(\frac{p \pi}{\gamma_{\lambda} d}\right)^{2}\right] \int_{A}|\psi|^{2} d a \quad \text { TM modes } \\
& U=\frac{d}{4} \mu\left(1+\left(\frac{p \pi}{\gamma_{\lambda} d}\right)^{2}\right] \int_{A}|\psi|^{2} d a \quad \text { TE modes }
\end{aligned}
$$

where $\mathrm{A}$ is the cross sectional area. For the $\mathrm{TM}_{010}$ mode this is:

$$
\begin{aligned}
U & =\frac{\epsilon_{0}}{2} E_{0}^{2} \int_{0}^{d} \int_{0}^{2 \pi} \int_{0}^{R}\left[J_{n}\left(\frac{X_{01}}{d} r\right)\right]^{2} r d r d \theta d z \\
& =\frac{\epsilon_{0}}{2} E_{0}^{2} \pi R^{2} d\left[J_{1}\left(X_{01}\right)\right]^{2}
\end{aligned}
$$

The power loss can be calculated by:

$$
P_{\text {loss }}=\frac{1}{2 \sigma \delta}\left[\oint_{C} d l \int_{0}^{d} d z|\mathbf{n} \times \mathbf{H}|_{\text {sides }}^{2}+2 \int_{A} d a|\mathbf{n} \times \mathbf{H}|_{\text {ends }}^{2}\right]
$$

where $\delta$ is the skin depth $\left(\sqrt{\frac{2}{\omega \mu \sigma}}\right)$, and $C$ is the circumference. For the $\mathrm{TM}_{010}$ mode this is:

$$
P_{\text {loss }}=\frac{\pi R(R+d)}{\sigma \delta}\left(\frac{E_{0}}{\mu_{0} c}\right)^{2}\left[J_{1}\left(X_{01}\right)\right]^{2}
$$

For a cavity with permeability of the walls $\mu_{c}$, the $Q$ of a cavity is given by:

$$
Q=\frac{\mu}{\mu_{c}} \frac{d}{\delta} \frac{1}{2\left(1+\xi_{\lambda} \frac{C d}{4 A}\right)}
$$

where $\xi_{\lambda}$ is a unitless number. This can be rewritten as:

$$
Q=\frac{\mu}{\mu_{c}}\left(\frac{V}{S \delta}\right) \times(\text { Geometrical factor })
$$

where $V$ is the volume of the cavity and $S$ is the total surface area. For the $\mathrm{TE}_{1,1,1}$ mode the geometrical factor is:

$$
\left(1+\frac{d}{R}\right) \frac{\left(1+0.343 \frac{d^{2}}{R^{2}}\right)}{\left(1+0.209 \frac{d}{R}+0.244 \frac{d^{3}}{R^{3}}\right)}
$$

For the $\mathrm{TM}_{010}$ mode:

$$
Q=\frac{R d}{\delta(R+d)}
$$

As a cavity can be modeled as a parallel RLC circuit, the quality factor can also be given as:

$$
Q=\omega_{0} R C=\omega_{0} \frac{L}{R}
$$




\section{CHAPTER 2}

\section{EXPERIMENT}

\subsection{MuCool Test Area}

The MuCool Test Area (MTA) is an experimental facility located at the end of Fermi National Accelerator Laboratory's linear accelerator (Linac). Construction of the experimental hall was completed during the fall of 2003. The MTA's purpose is to test experimental components for muon cooling [101]. At present the main focus is on research and development of technology that would allow RF cavities to operate in strong magnetic fields.

This task is being approached on a variety of paths. The experimental program utilizing the MTA facility involves several test devices. One, the HPRF cavity, is the topic of this thesis. Another is a vacuum pillbox cavity operated at $805 \mathrm{MHz}$ with removable electrodes made of various materials [102]. A new cavity with removable end plates is being fabricated to replace that one. Another $805 \mathrm{MHz}$ pillbox cavity can be operated both under vacuum and high pressurize gas [103]. There is also a $201 \mathrm{MHz}$ vacuum pillbox cavity [28].

\subsection{Beamline}

The MTA beamline was completed during the Fall of 2008. The goal was to provide a beam intensity $\left(O\left(10^{13}\right.\right.$ protons/pulse $\left.)\right)$ approaching the bunch intensity for a Muon Collider. As of Spring 2013 it is the only experimental facility at Fermilab to take beam directly from the Linac. In addition to providing beam for muon cooling $\mathrm{R} \& \mathrm{D}$, a second mode of operation is possible, to provide measurements of transverse emittance and momentum spread for the Linac. In the so-called emittance mode, the Linac beam is sent to the MTA hall and stopped in a beam absorber upstream of the experimental setup. The momentum spread of the beam when it exits the Linac is 
$\Delta p / p=0.005$ [104]. The beam stop rotates in and out of the beamline, and so in experimental mode the beam passes through the beam stop assembly, before passing through a $50.8 \mu \mathrm{m}$ thick titanium window and into the experimental area.

The Fermilab Linac beam starts in the form of $\mathrm{H}^{-}$from a $25 \mathrm{keV}$ ion source. The $\mathrm{H}^{-}$are accelerated to $750 \mathrm{keV}$ by a Cockroft Walton generator, after which they enter a drift tube (Alvarez) Linac operating at 201.24 MHz where they reach $116 \mathrm{MeV}$. From there they enter an $805 \mathrm{MHz}$ side coupled cavity Linac where they reach their final kinetic energy of $401 \mathrm{MeV}$ [105]. This final section of the Linac has specifications given in Tab. 2.1. 
Table 2.1. Fermilab $400 \mathrm{MeV}$ Linac specifications.

\begin{tabular}{|c|c|c|}
\hline Parameter & Value & Units \\
\hline Initial kinetic energy & 116.54 & $\mathrm{MeV}$ \\
\hline Final kinetic energy & 401.56 & $\mathrm{MeV}$ \\
\hline Length & 64.3 & $\mathrm{~m}$ \\
\hline RF frequency & 805 & $\mathrm{MHz}$ \\
\hline Avg. beam current & 50 & $\mathrm{~mA}$ \\
\hline Beam pulse length & $<100$ & $\mu \mathrm{s}$ \\
\hline Repetition rate & 15 & $\mathrm{~Hz}$ \\
\hline Accelerating phase & -32 & $\operatorname{deg}$ \\
\hline Number of modules & 7 & \\
\hline Number of sections/module & 4 & \\
\hline Number of RF cells/section & 16 & \\
\hline Transverse focusing scheme & FODO & \\
\hline Avg. transverse phase advance/cell & 79 & $\operatorname{deg}$ \\
\hline Avg. axial field & $7.07-8.04$ & $\mathrm{MV} / \mathrm{m}$ \\
\hline Max. surface field & 37.1 & $\mathrm{MV} / \mathrm{m}$ \\
\hline Cavity bore radius & 1.5 & $\mathrm{~cm}$ \\
\hline Quadrupole magnetic length & 7.0 & $\mathrm{~cm}$ \\
\hline Quadrupole poletip field & 5.26 & $\mathrm{kG}$ \\
\hline Quadrupole bore radius & 2.0 & $\mathrm{~cm}$ \\
\hline
\end{tabular}


The Linac has the capability of sending the beam to four locations: the booster, for use in various experiments; the MTA; the Neutron Therapy Facility, for cancer treatment; or a beam dump. The transport line from the Linac to the MTA hall is shown schematically in Fig. 2.1. The beam enters at the bottom right, where two C-magnets steer the beam to the MTA transfer line and a chopper discards the head and tail of the beam in order to get the desired quality and pulse length.

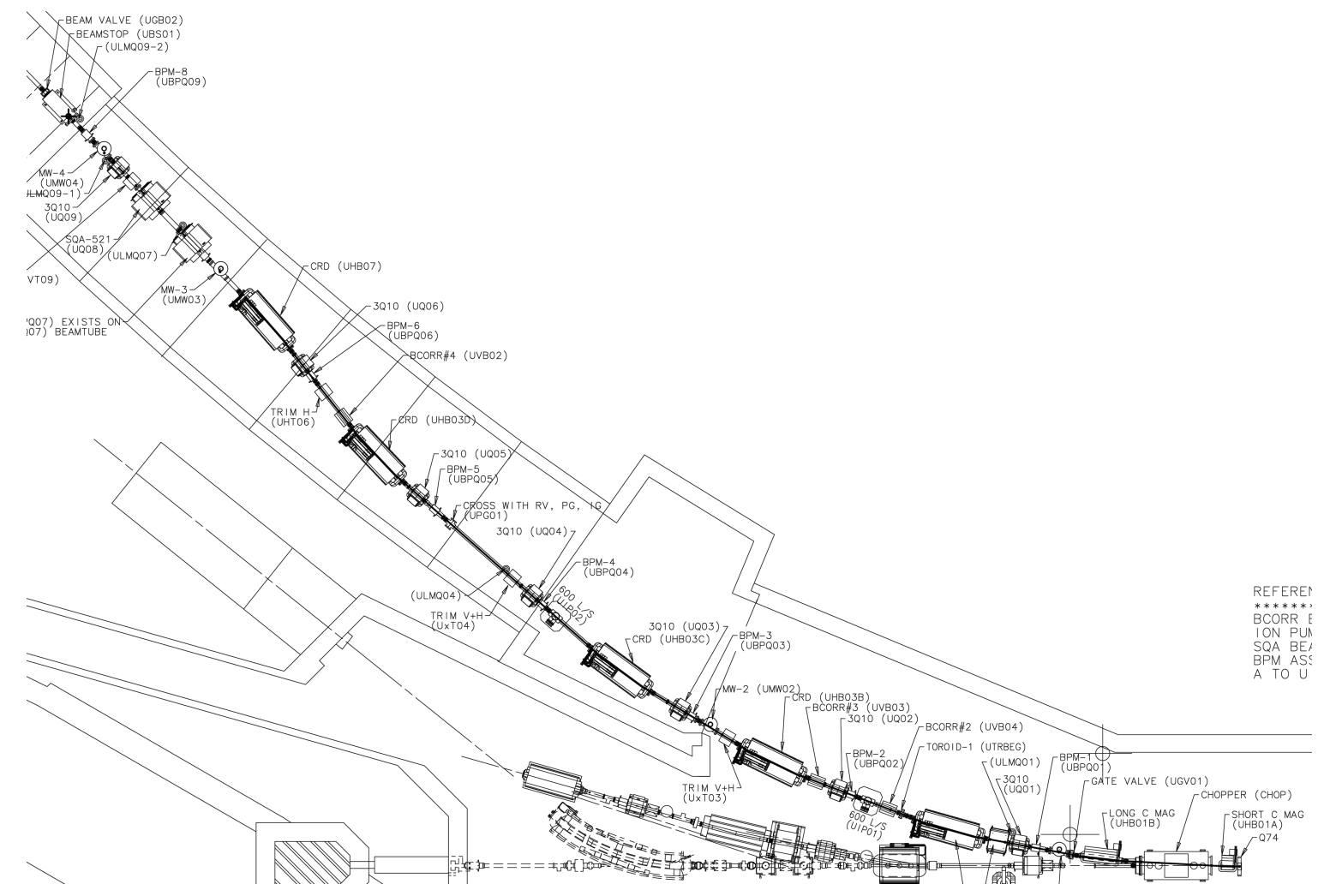

Figure 2.1. Linac hall engineering drawing. The beam comes from the Linac (bottom right) and goes to the MTA hall (top left).

The first Linac toroid, beam position monitor (BPM) and multiwire detector are placed shortly after the C-magnets. A series of dipole and quadrupole magnets steer and focus the beam to the shield wall between the Linac and MTA experimental hall. At this point there is a beamstop, to prevent the beam from entering the hall, if desired. The hall is slightly elevated with respect to the Linac, so the beam enters 
the hall at an angle with respect to the floor.

Figure 2.2 depicts the beamline in the MTA hall. The beam enters the hall from the right, after which it passes through a quadrupole triplet and two vertical bending magnets providing the final focusing and steering. There are two Linac toroids, three multiwire detectors (one at the end of the beampipe is not shown), and two BPMs in the hall to provide instrumentation. After exiting the beampipe vacuum window, the beam passes through $\sim 1 \mathrm{~m}$ of air before entering the solenoid magnet.

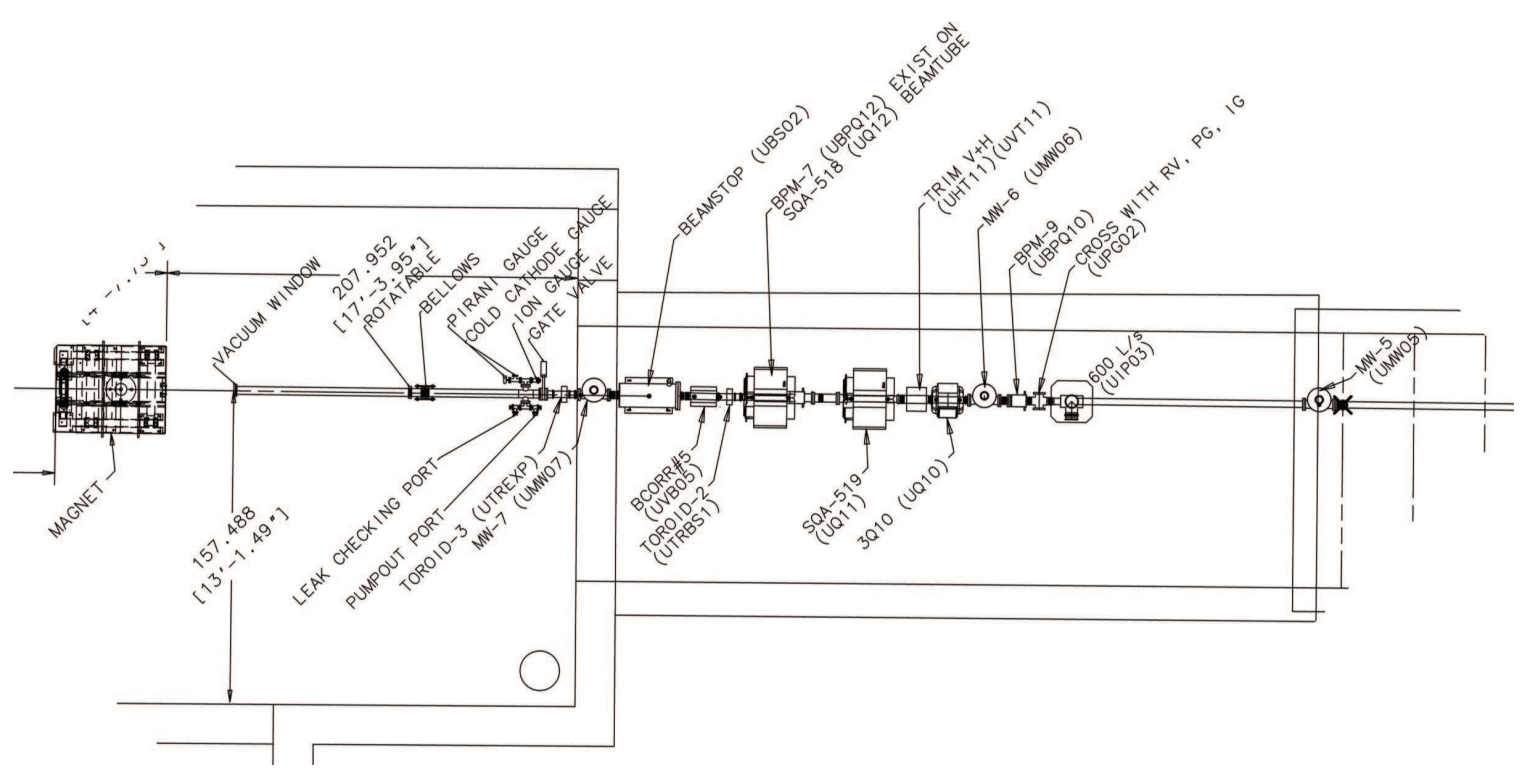

Figure 2.2. MTA hall engineering drawing.

When running in experimental mode, the MTA receives one beam pulse per minute. The clock signal from the Linac C-magnets that indicates the magnets are being energized is sent to the MTA controls for use as a trigger, to synchronize the timing of the beam and instrumentation.

\subsection{Experimental Setup}

The experimental setup is shown in Fig. 2.3. The photo shows the view looking 
downstream along the beampipe toward the solenoid magnet from the approximate location of the CCD camera. The engineering drawing shows the setup inside the magnet. The beam enters from the right, hitting the scintillating screen. The beam passes through the first collimator, made of stainless steel, is $15.24 \mathrm{~cm}$ in diameter and $10.16 \mathrm{~cm}$ long, and has a through hole $20.0 \mathrm{~mm}$ in diameter. The first of two toroids is mounted on the upstream face of the second collimator. The second collimator is also made of stainless steel, is $15.24 \mathrm{~cm}$ in diameter and $20.32 \mathrm{~cm}$ long, and has a $4 \mathrm{~mm}$ through hole. The second toroid is mounted on the downstream face of the second collimator. From here the beam passes through the HPRF cavity and is absorbed in a beam absorber. The entire apparatus is mounted on rails that are secured in place inside the bore of the magnet.
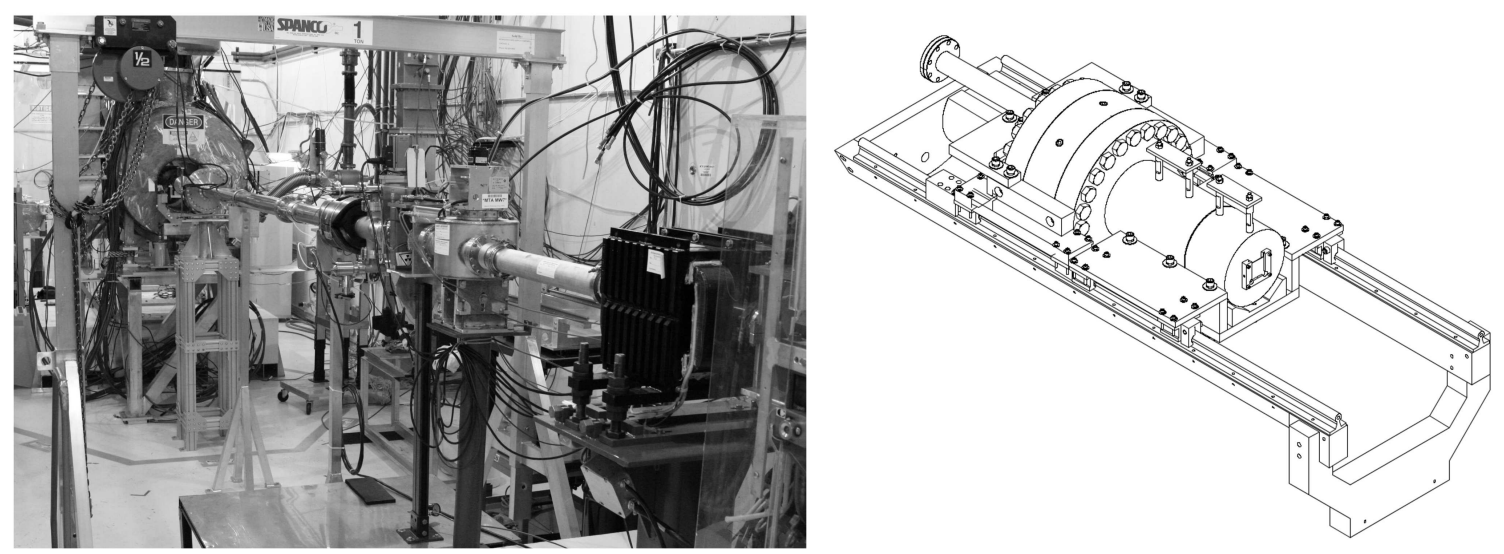

Figure 2.3. Looking downstream along the beampipe toward the experimental setup (left). Engineering drawing of the experimental apparatus located inside the solenoid magnet (right). The beam enters from the right. In the drawing, the scintillating screen can be seen on the upstream face of the first collimator. The upstream toroid is mounted on the upstream face of the second collimator (not shown). The downstream toroid is mounted on the downstream face of the second collimator (not shown). The HPRF cavity and beam absorber are also shown.

An array of instrumentation is employed while collecting data. A CCD camera records a video of the beam striking a scintillating screen for use in beam tuning. Two toroids measure the beam intensity before and after the final collimator. Electric and 
magnetic pickup probes measure the RF electric field inside the cavity. A photomultiplier tube (PMT) and silicon photomultiplier (SiPM) measure light produced in the cavity, sent along an optical fiber. Finally, a series of directional couplers measure the forward and reflected power to and from the cavity at various locations.

2.3.1 CCD Camera / Scintillating Screen. For purposes of beam tuning and positioning, a PixelLINK USB CCD camera and Chromox-6 scintillation screen were used [106]. The camera was positioned upstream of the experimental apparatus and off of the beam axis, so as to have a clear view of the screen, which was mounted on the upstream face of the first collimator. For each beam pulse, a video of the screen was recorded so that the positioning of the beam relative to the hole in the collimator could be estimated. Figure 2.4 shows the screen and a still image from a video of the beam pulse hitting the screen.

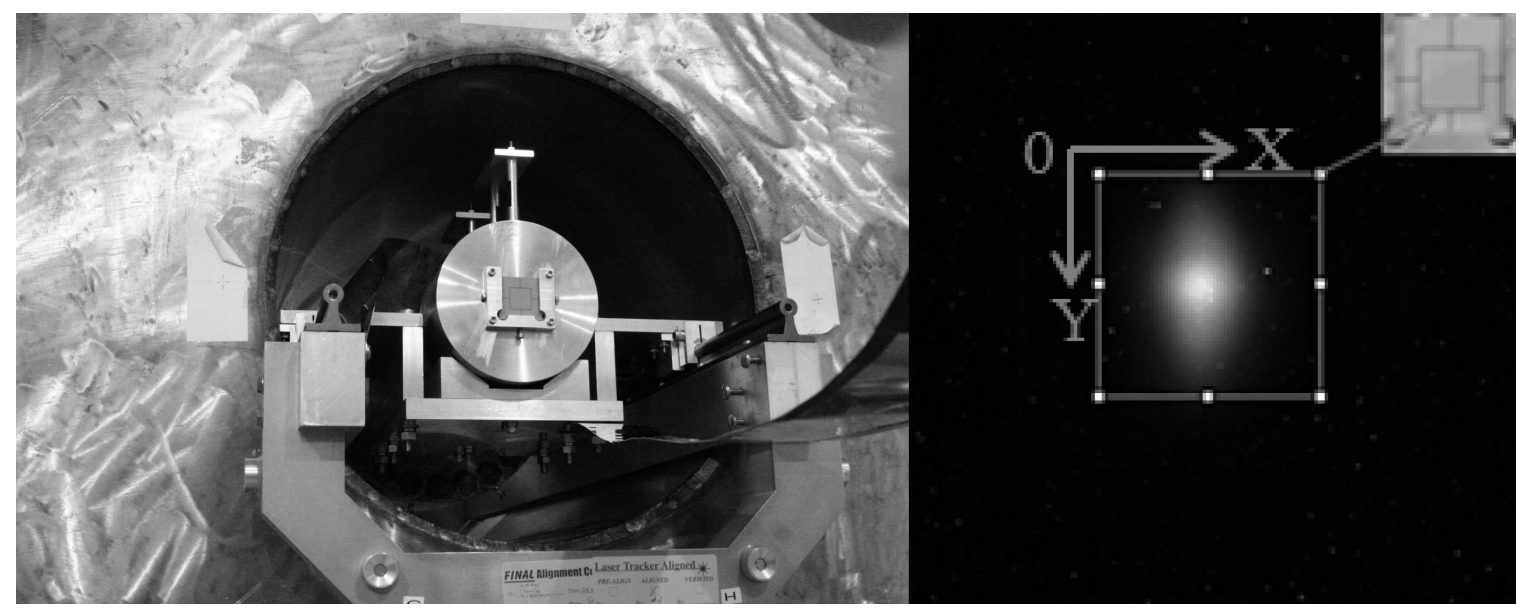

Figure 2.4. Left: The scintillating screen was mounted on the upstream face of the first collimator. Right: A still image from a video of the beam pulse hitting the screen.

Because the screen is upstream of the collimators, it cannot be used to make a direct measurement of the beam intensity incident on the cavity. Rather, from knowing the alignment of the screen relative to the holes in the collimators and by 
fitting the intensity of scintillation light from the screen, the transmission through the collimators could be estimated. To accomplish this, a still image of the screen before beam passage was subtracted from the first frame of the screen being illuminated by the beam. The CCD camera records video at 10 frames per second, and the first frame was selected to minimize any effect of nonlinearity in the decay of the light from the screen. The resulting background-subtracted image was fit with a Gaussian function based on the intensity of light recorded. To minimize saturation of the camera, a neutral density filter was used. Without magnetic field, the transmission measured by the CCD/screen and toroids agree to within 5\% [107]. A number of factors increase the error on the beam intensity using this method: possible saturation of the CCD camera, fitting of the image, and relying on the beam intensity measured several meters upstream of the experimental apparatus.

2.3.2 Beam Intensity Measurement. Measurements of the beam intensity were made at multiple locations along the beamline for each beam pulse received by the experiment. Each measurement was made using a current transformer in the form of a toroidal core with wire wrapped around it. The Linac group of Fermilab's Accelerator Division operated three toroids, one placed a small distance downstream of the $\mathrm{C}$ magnet in the Linac hall, one immediately downstream of the final quadrapole triplet in the MTA hall, and one a small distance downstream of the emittance beam stop in the MTA hall. These locations are indicated in Figures 2.1 and 2.2, (labeled Toroid 1, Toroid 2, and Toroid 3, respectively). Several toroids were built and calibrated for the purpose of determining the final beam intensity at the entrance of the HPRF cavity. One was placed between the first and second collimators of the MTA experimental apparatus, the other immediately downstream of the second collimator (see upstream toroid and downstream toroid in Figure 2.3).

Current transformers operate based on Faraday's law of induction. For a wire 
wound around a magnetic core, the induced electromotive force in the wire is equal to the time rate of change of the magnetic flux in the core multiplied by the number of turns of the wire:

$$
\varepsilon=-N \frac{d \Phi_{B}}{d t}
$$

The magnetic flux is given by:

$$
\Phi_{B}=\int_{S} \vec{B} \cdot d \vec{A}
$$

If there are two wires wound around an ideal core (i.e. there is no flux leakage) then the magnetic flux through both windings is the same and:

$$
\begin{aligned}
\varepsilon_{1} & =-N_{1} \frac{d \Phi_{B}}{d t} \\
\varepsilon_{2} & =-N_{2} \frac{d \Phi_{B}}{d t}
\end{aligned}
$$

The two EMFs are related by the number of windings of each coil:

$$
\frac{\varepsilon_{1}}{\varepsilon_{2}}=\frac{N_{1}}{N_{2}}
$$

Ampère's law states that a current produces a magnetic field according to:

$$
\oint_{C} \vec{B} \cdot d \vec{l}=\mu_{0} I
$$

where $\mu_{0}$ is the permeability of free space. The current can thus be found by integrating the magnetic field around a closed loop. If a beam of charged particles passes through a toroid, the induced magnetic field in the core produces a magnetic flux that is seen by the wire wrapped around it. The current induced in the wire must be proportional to the beam current, and energy conservation implies $P_{1}=I_{1} V_{1}=P_{2}=I_{2} V_{2}$. Using Eq. 2.5:

$$
I_{2}=\frac{N_{1}}{N_{2}} I_{1}
$$

The beam can be thought of as a current with a single turn, so the induced current in the wire is equal to the beam current divided by the number of turns of the wire. 
The toroid assembly forms a series RL circuit and, based on Kirchhoff's second law,

$$
V=I R+L \frac{d I}{d t}
$$

where $L$ is the inductance of the toroid core.

A total of four toroids were built during the course of data taking, two for the first data run and two for the second data run. For each run the toroids were placed just upstream of the HPRF cavity so that an accurate measurement of the number of protons incident on the cavity could be made. All toroids were made using manganese zinc ferrite cores manufactured by Fair-Rite Products Corp [108]. For Run I, each toroid consisted of a single core wound with ten turns of plastic coated copper wire connected to a BNC connector. For Run II, the upstream toroid consisted of a single core wound with five turns of plastic coated copper wire connected to a BNC connector. The downstream toroid consisted of three cores wound with five turns of plastic coated copper wire connected with a BNC connector. The Run II toroids were placed in an aluminum housing to provide shielding from stray particles and allow easy mounting on the downstream collimator. The housing consisted of concentric rings joined on the ends by a circular plate. A small gap between the inner ring and one end plate was left so as to not completely insulate the toroids electromagnetically (see Fig. 2.5).

A calibration of the two MTA toroids was performed and the inductance of each measured. Because of the tight confines of the bore of the magnet in which the experimental apparatus was placed, the measurements could not be performed in situ. The calibration was performed by connecting a wire and resistor to make the shielding a closed circuit, effectively creating a single turn primary winding. A function generator was connected and a $100 \mathrm{kHz}$ square wave (there was no suitable function generator that could produce a $200 \mathrm{MHz}$ signal available) was sent through the primary winding while the response of the toroid along with the function generator 


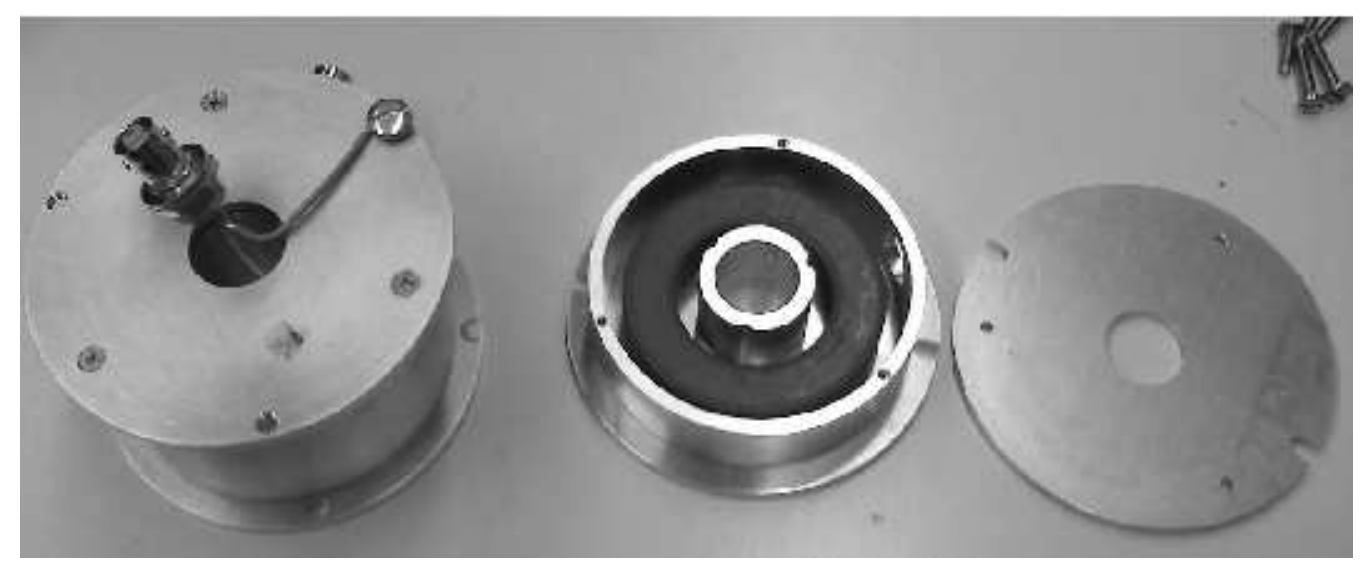

Figure 2.5. Housing for the upstream (right) and downstream (left) toroids. The upstream housing has one end plate removed, showing the ferrite core inside. The downstream one has a connector attached to the housing, making the housing a closed circuit, allowing for a current to be run through the aluminum case.

signal was measured on an oscilloscope. The resistor could be changed in order to vary the primary current. Fig. 2.6 shows the $8.72 \mathrm{~V}$ function generator signal and the downstream toroid response. A fit to the exponential decay of the toroid signal was made in order to extract the inductance. The measured time constant was $4.15 \mu \mathrm{s}$. The scope was terminated in $50 \Omega$, giving an inductance of $207.5 \mu \mathrm{H}$. The measured inductance of each toroid is listed in Table 2.2. A $500 \Omega$ resistor in series with the function generator gave a primary current of $17.4 \mathrm{~mA}$. Using Eq. 2.7 to calculate the expected current through the secondary winding of five turns, one would expect a $174 \mathrm{mV}$ signal from the toroid. The measured signal was $179 \mathrm{mV}$. This is a $3 \%$ difference, which will contribute to the uncertainty in the beam current measurement described later. 
Table 2.2. Toroid Inductance Measurements.

\begin{tabular}{ccc}
\hline \hline Run & Toroid & Inductance $(\mu H)$ \\
\hline 1 & Upstream & 326.8 \\
1 & Downstream & 331.5 \\
2 & Upstream & 73.6 \\
2 & Downstream & 207.5 \\
\hline
\end{tabular}

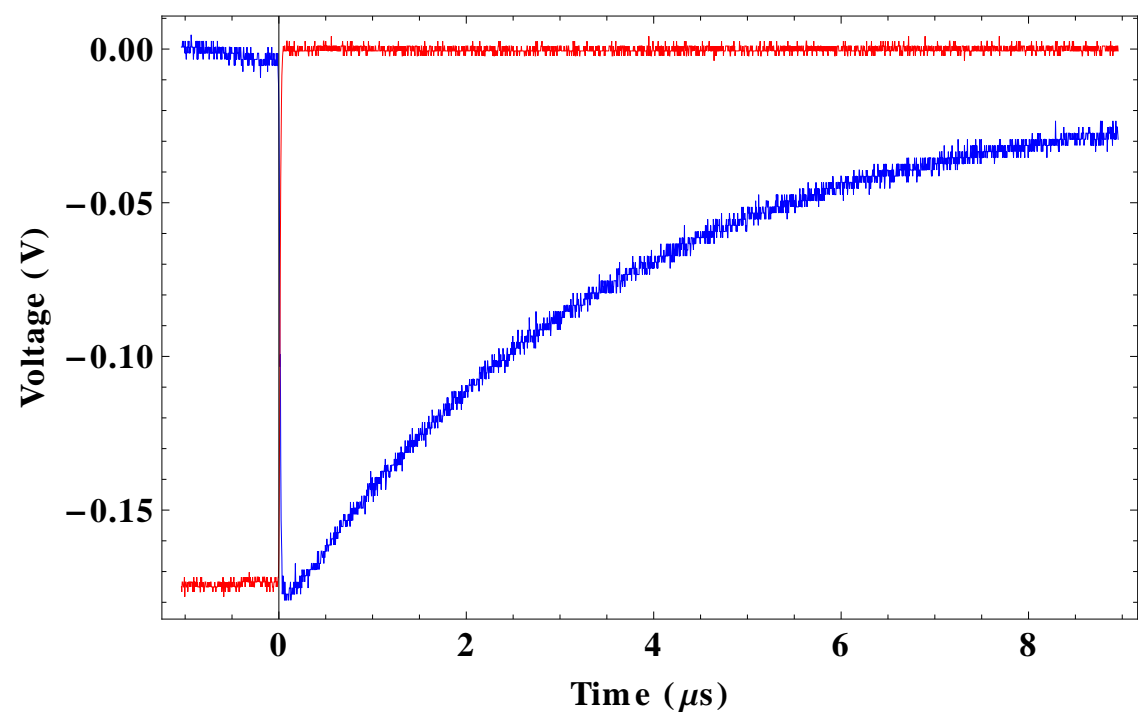

Figure 2.6. Downstream toroid calibration data. Function generator signal divided by 50 in red, toroid signal in blue. 
The three toroids maintained by the Linac group were all commercially produced by Pearson Electronics [109]. Fermilab's Accelerator Division was responsible for their calibration, which was performed in August 2011, before the second beam run. These toroids were read out by Fermilab's Accelerator Control Network (ACNET) [110]. These data were available on a pulse-by-pulse basis and were archived for later retrieval. Their output was number of protons per beam pulse, and Fig. 2.7 shows the data from April 18, 2012.

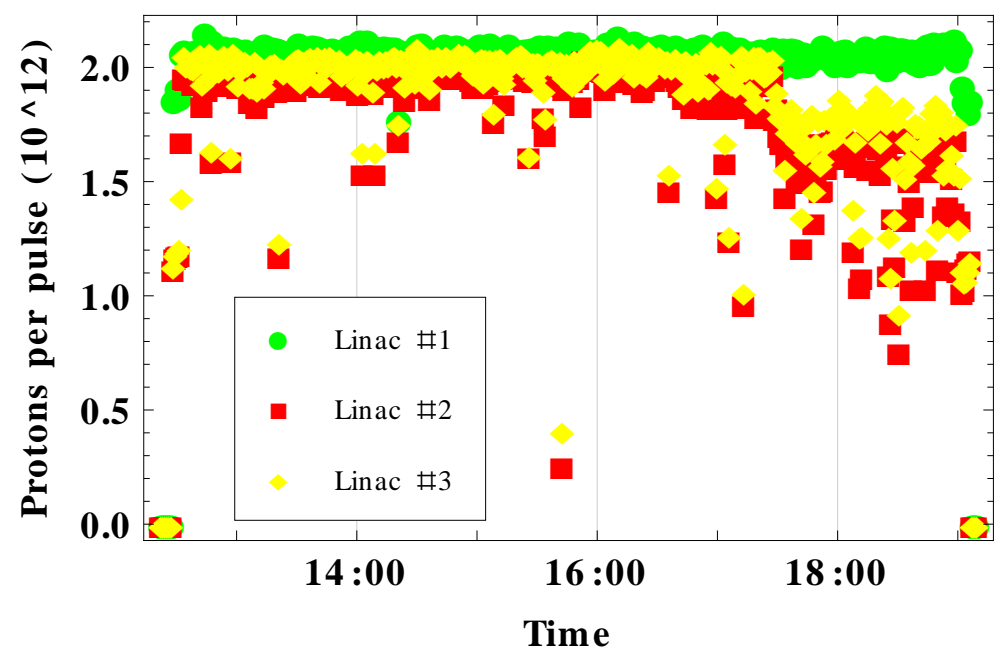

Figure 2.7. Linac toroid data from April 18, 2012. The axes are beam intensity versus time. UTR01 is in green and is the most upstream toroid, UTR02 is in red, and UTR03 is in yellow and is the most downstream toroid. The beam intensity is roughly $2 \times 10^{12}$ protons per pulse.

The data from the Linac toroids combined with the MTA toroids and CCD camera / scintillating screen were used extensively during beam tuning for purposes of positioning and focusing the beam. The highest beam intensity was achieved by centering the beam on the hole in the collimator and using the tightest focus. Unfortunately it was not possible to defocus the beam as much as one would have liked, and so smaller beam intensities were achieved through a combination of defocusing the beam and moving it off the center of the collimator hole, thereby allowing only the halo of the beam to reach the HPRF cavity. 
2.3.3 HPRF Cavity. The HPRF cavity was designed to be a test cell with which to study RF breakdown. Copper electrodes were used to enhance the peak electric field on-axis and localize the area of potential breakdown. As such, it had a small stored energy. This made the effect of plasma loading easier to measure. Figure 2.8 shows an engineering drawing of the cavity. All inlets and instrumentation were located on the downstream face of the cavity. RF power was fed inductively through the port in the top left of the diagram. Gas was filled and vented through a port at the bottom left of the diagram. An electric and magnetic pickup feedthrough and three optical feedthroughs are not shown in the diagram. The upstream face and electrode were drilled to minimize the amount of material the beam had to interact with before entering the cavity (the thickness of material for each was $3.175 \mathrm{~mm}$ ).

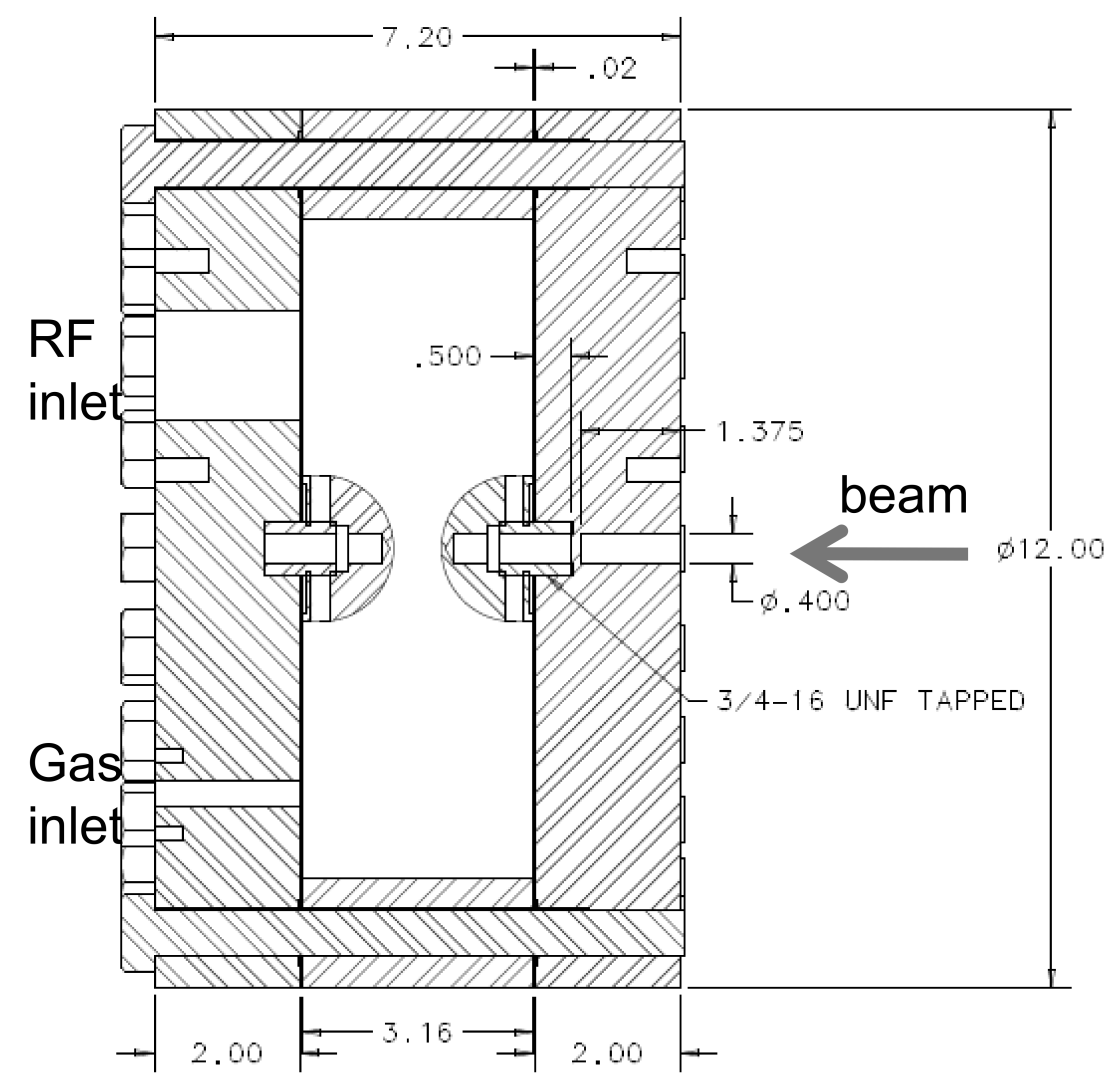

Figure 2.8. Engineering drawing of the HPRF cavity. The RF coupling port and gas fill line are shown. Optical and probe ports are not shown, but are located on the same face as the RF port. 
The cavity was made of copper coated stainless steel. As can be seen in Fig. 2.8, there were three pieces, an upstream and downstream plate and a circular body, held together by 24 bolts. The plates were $5.05 \mathrm{~cm}$ thick and the walls were $2.54 \mathrm{~cm}$ thick. A pressure flange between each plate and the body sealed the cavity. The bolts allowed safe operation up to 1520 psi gas pressure. Figure 2.9 (left) shows the downstream plate of the cavity. The RF power input, electric and magnetic pickup probes, three optical ports, and gas inlet are visible. The interior of the downstream plate is shown (right). The electrode is visible here as well. Table 2.3 lists the parameters of the cavity.

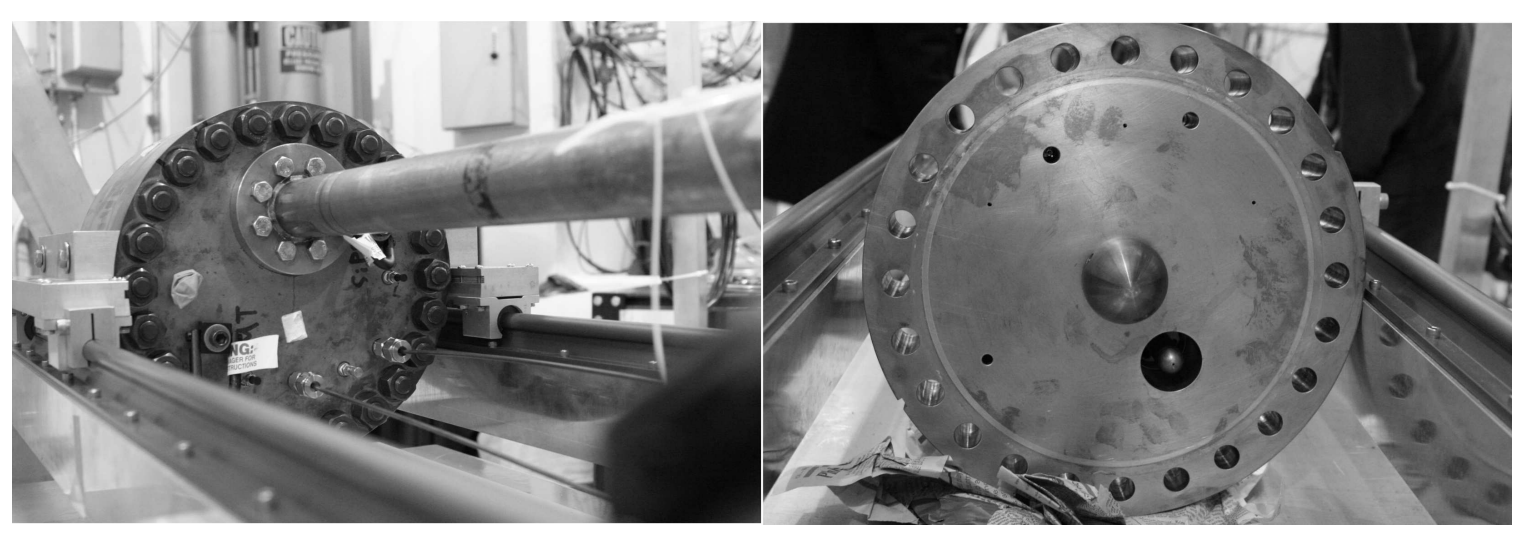

Figure 2.9. HPRF Cavity. Left: The exterior of the downstream plate. Starting at the top with the coaxial RF power input and moving clockwise are: an optical port, a pickup port, an optical port, a pickup port, an optical port, and the gas inlet port. Right: The interior of the downstream plate. In addition to the ports, one copper electrode is visible. 
Table 2.3. HPRF cavity parameters.

\begin{tabular}{lrl}
\hline \hline Parameter & Value & Units \\
\hline Resonant frequency (filled w/ $\mathrm{H}_{2}$ gas) & $801.3-808.5$ & $\mathrm{MHz}(1520-300 \mathrm{psi})$ \\
Inductance & 26.08 & $\mathrm{nH}$ \\
Stored energy at $1 \mathrm{MV} / \mathrm{m}$ & 3.98 & $\mathrm{~mJ}$ \\
Stored energy at $25 \mathrm{MV} / \mathrm{m}$ & 2.49 & $\mathrm{~J}$ \\
Unloaded Q & $14,200-13,900 \quad($ at $801-808 \mathrm{MHz})$ \\
Loaded Q & $6,900-6,400$ & $($ at $801-808 \mathrm{MHz})$ \\
R & $52.1-56.7$ & $\Omega($ at $801-808 \mathrm{MHz})$ \\
Cavity interior length & 8.13 & $\mathrm{~cm}$ \\
Cavity interior diameter & 22.86 & $\mathrm{~cm}$ \\
Electrode gap separation & $1.77 \quad \mathrm{~cm}$ \\
\hline
\end{tabular}

The electric field distribution in the cavity has been simulated using Superfish [111]. Figure 2.10 shows the longitudinal and radial components vs. $z$ (the longitudinal axis).

2.3.4 RF Power. RF power is fed to the HPRF cavity through a series of rectangular and coaxial waveguides by a $4 \mathrm{MW}$ klystron. Klystrons are microwave tubes that take a DC input and output a high power RF signal. Klystrons work by taking a beam of electrons produced by an electron gun (a cathode and anode with a beam modulating electrode in between) and sending it through a series of RF cavities. The beam is bunched at the upstream end, while the amplified RF power is extracted at the downstream end. Typical gains are $\sim 20 \mathrm{~dB}$ for two cavities and $80-90 \mathrm{~dB}$ for 

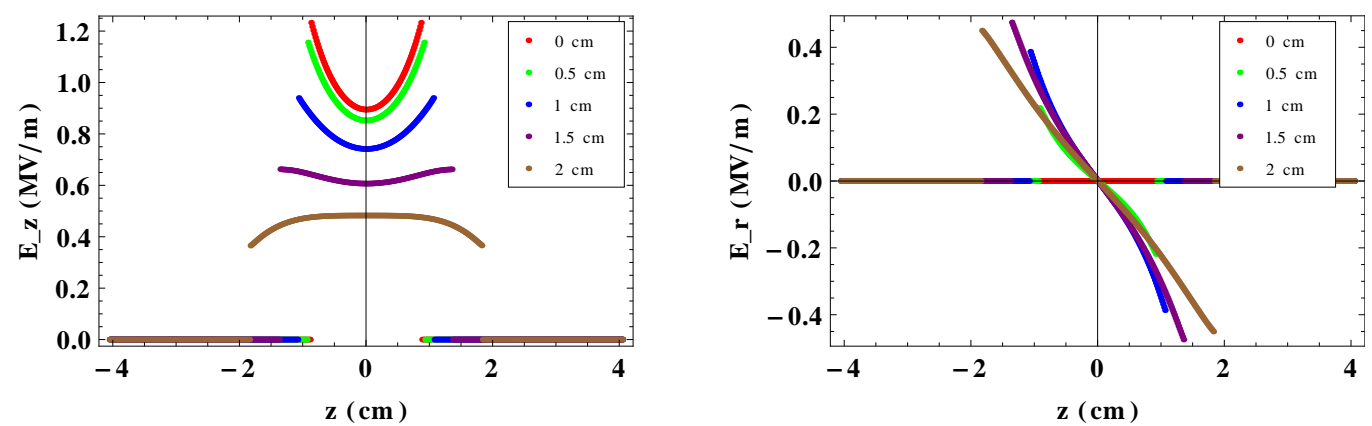

Figure 2.10. Electric field vs. $z$ inside the HPRF cavity. $E_{z}$ is on the left. The colors correspond to different radii: Red $=0.0 \mathrm{~cm}$, Green $=0.5 \mathrm{~cm}$, Blue $=1.0 \mathrm{~cm}$, Purple $=1.5 \mathrm{~cm}$ and Brown $=2.0 \mathrm{~cm}$. At $r=0 \mathrm{~mm}$ the gap spacing is $1.77 \mathrm{~cm}$. The average $E_{z}$ has been normalized to one. $E_{r}$ is plotted on the right.

four cavities [112]. The DC to RF power conversion is about $30-50 \%$.

A series of rectangular and coaxial waveguides carry the power to the cavity. One of two devices was used to provide cleaner RF signals by minimizing reflections of the RF wave. The first was a circulator. A circulator is a type of power divider (or T-junction) with three ports. In general, the voltage amplitude of an incident wave on port $n, V_{n}^{+}$, is related to the voltage amplitude of the reflected wave by port $n$, $V_{n}^{-}$, by $[112]$ :

$$
\left[\begin{array}{c}
V_{1}^{-} \\
V_{2}^{-} \\
\vdots \\
V_{n}^{-}
\end{array}\right]=\left[\begin{array}{cccc}
S_{11} & S_{12} & \cdots & S_{1 n} \\
S_{21} & \ddots & & \vdots \\
\vdots & & \ddots & \\
S_{n 1} & \cdots & & S_{n n}
\end{array}\right]\left[\begin{array}{c}
V_{1}^{+} \\
V_{2}^{+} \\
\vdots \\
V_{n}^{+}
\end{array}\right]
$$

In this case, with three ports and a nonreciprocal matrix $\left(S_{i j} \neq S_{j i}\right)$, the ports being matched, the scattering matrix reduces to:

$$
\left[\begin{array}{ccc}
0 & S_{12} & S_{13} \\
S_{21} & 0 & S_{23} \\
S_{31} & S_{32} & 0
\end{array}\right]
$$

For the circulator to be lossless, the scattering matrix must be unitary, which implies 
either $S_{12}=S_{23}=S_{31}=0$ or $S_{21}=S_{32}=S_{13}=0$. This gives a scattering matrix of:

$$
\left[\begin{array}{lll}
0 & 0 & 1 \\
1 & 0 & 0 \\
0 & 1 & 0
\end{array}\right] \quad \text { or } \quad\left[\begin{array}{lll}
0 & 1 & 0 \\
0 & 0 & 1 \\
1 & 0 & 0
\end{array}\right]
$$

Therefore power flows in a circular fashion between the three ports (see Fig. 2.11). Power is input in port 1 and the cavity is connected to port 2. A load is connected to port 3 to dissipate any power sent back from the cavity. The load that was available for the circulator allowed operation of the cavity up to a gradient of $30 \mathrm{MV} / \mathrm{m}$. Figure 2.12 shows the circulator installed in the MTA. The input is connected to the rectangular waveguide, the output connected to the coaxial waveguide which is connected to the cavity, and the third port contains the load (and a directional coupler). Note that the presence of a magnetic field greatly reduces the performance of a circulator.

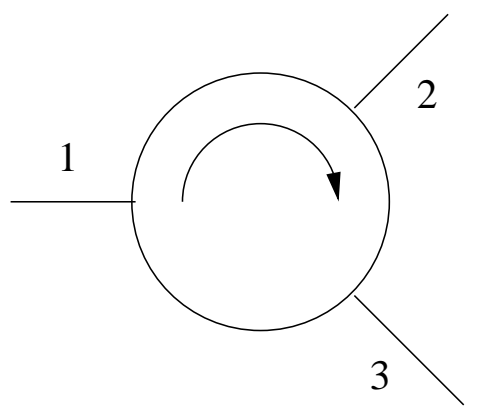

Figure 2.11. Circulator schematic.

In order to operate the cavity at a gradient $>30 \mathrm{MV} / \mathrm{m}$, a hybrid device was used. A hybrid is very similar to a directional coupler (see Sec. 2.3.7). It was used for isolation by attaching loads to the coupled and isolation ports. Figure 2.13 shows the hybrid installed in the MTA hall. 


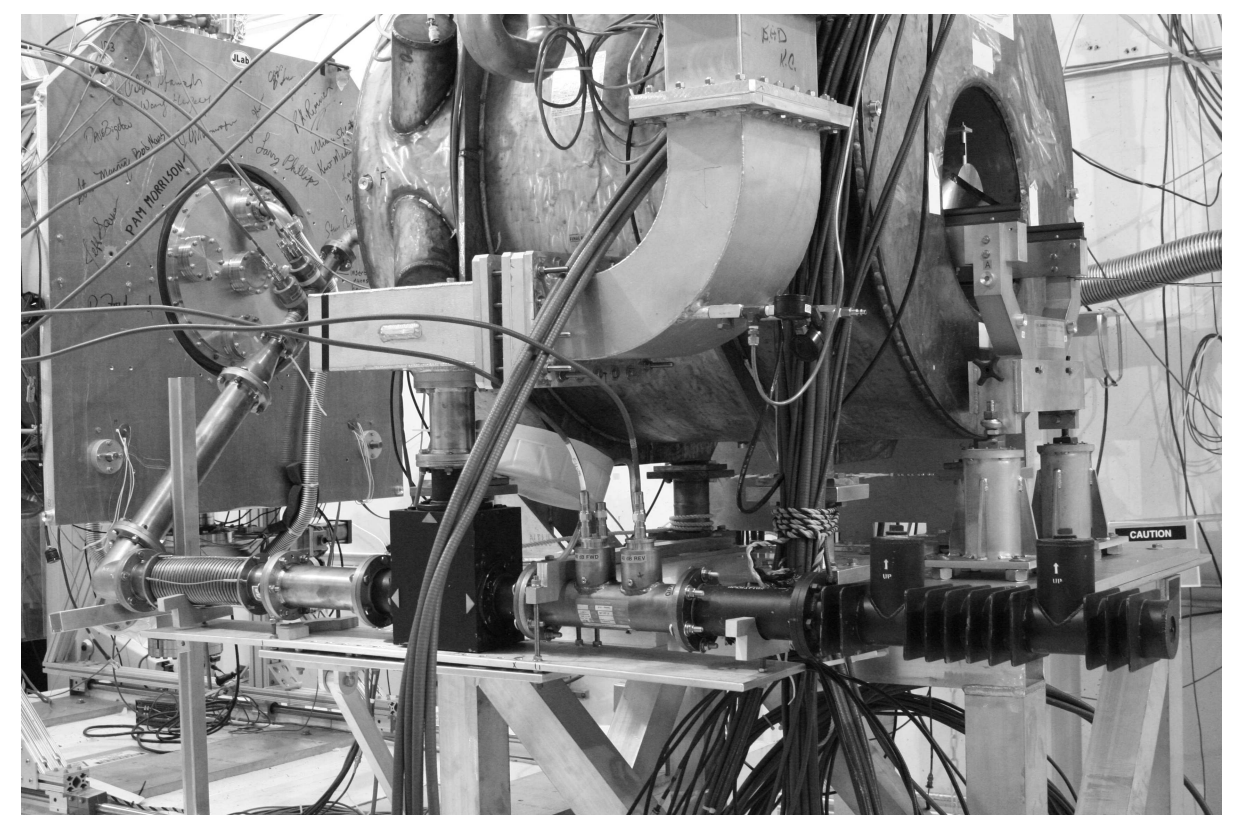

Figure 2.12. The circulator installed in the MTA hall. Port 1 is connected to the rectangular waveguide, port 2 is connected to the coaxial waveguide going to the cavity, and port 3 is connected to a load.

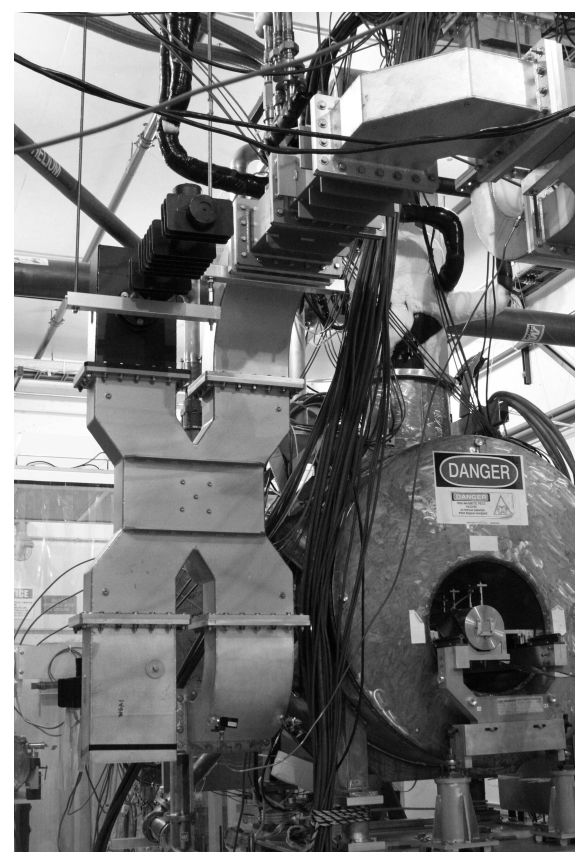

Figure 2.13. The hybrid installed in the MTA hall. Port 1 is connected to a rectangular waveguide, port 2 is connected to the coaxial waveguide going to the cavity, port 3 is connected to a load, and port 4 is also connected to a load.

2.3.5 Calibration. A Hewlett Packard 8753C network analyzer and Hewlett 
Packard 85047A S-Parameter Test Set were used to calibrated the electric and magnetic pickup probes. The entire system (cavity, cables, attenuators) was included in the calibration. To accomplish this, the cavity was excited at frequencies ranging from 801.72 to $808.46 \mathrm{MHz}$ and the response was measured with the network analyzer. The average calibrations for the electric and magnetic pickups are $35.5 \mathrm{MV} / \mathrm{m} / \mathrm{V}$ and $55.47 \mathrm{MV} / \mathrm{m} / \mathrm{V}$, respectively.

The signals from each instrument must pass along $\sim 100 \mathrm{~m}$ of cable from the MTA hall to the MTA controls, located in the Linac gallery. To minimize signal loss, 1/4-inch and 1/2-inch heliax cable, which has low attenuation (0.154 and $0.0840 \mathrm{~dB} / \mathrm{m}$, respectively) was used. The step response of the cable vs. time is given by [113]:

$$
f(t)=1-\operatorname{erf}\left(\frac{b l}{\sqrt{2 t}}\right)
$$

where erf is the error function and $b l=1.45 \times 10^{-6} \times$ the total attenuation of the cable. Figure 2.14 shows the cable response function vs. time. The attenuation of each cable was measured, and is listed in Tab. 2.4. Table 2.5 shows the cable assignments.

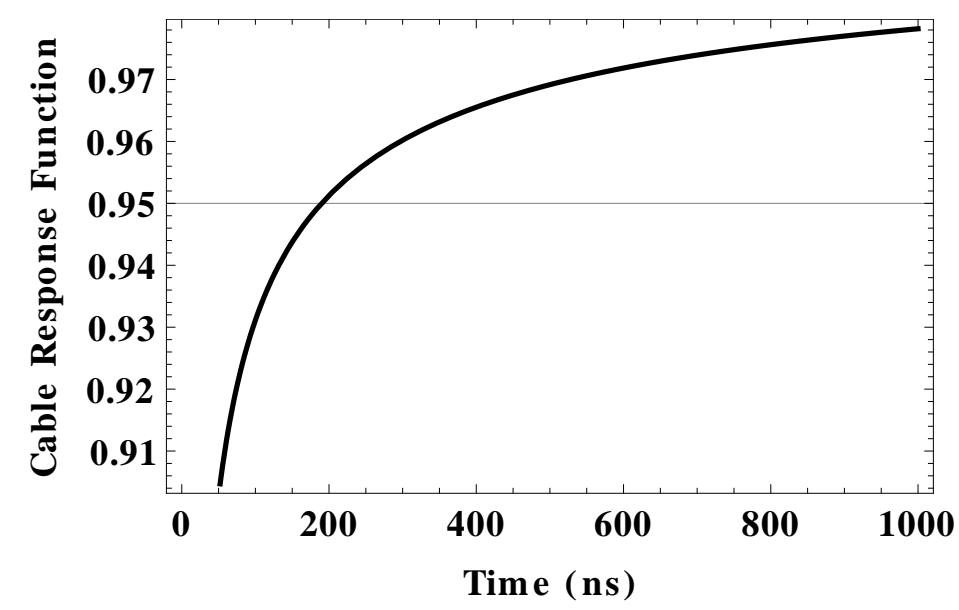

Figure 2.14. Cable response function vs. time. It takes nearly $200 \mathrm{~ns}$ to reach $95 \%$ of the total signal.

The relative timing between signals is also very important, as data were recorded at $50 \mathrm{ps}$ timing bins. To measure the time delay between cables, the system 
Table 2.4. Signal cable attenuation.

\begin{tabular}{ll}
\hline \hline Cable Designation & Attenuation $(\mathrm{dB})$ \\
\hline S1-01-L & -30.35 \\
S1-02-L & -29.25 \\
S2-07-S & -19.015 \\
S2-08-S & -18.848 \\
\hline
\end{tabular}

shown in Fig. 2.15 was used. A picosecond laser diode system (PiLas) sent an optical signal through an optical port in the HPRF cavity, where, after traveling through an optical fiber, it was seen by a PMT and sent to a digital oscilloscope. At the same time a signal was sent through another cable and recorded on the same oscilloscope. Each cable was measured, using the PMT signal as reference, thereby measuring the relative delays. The delays are given in Tab. 2.6.

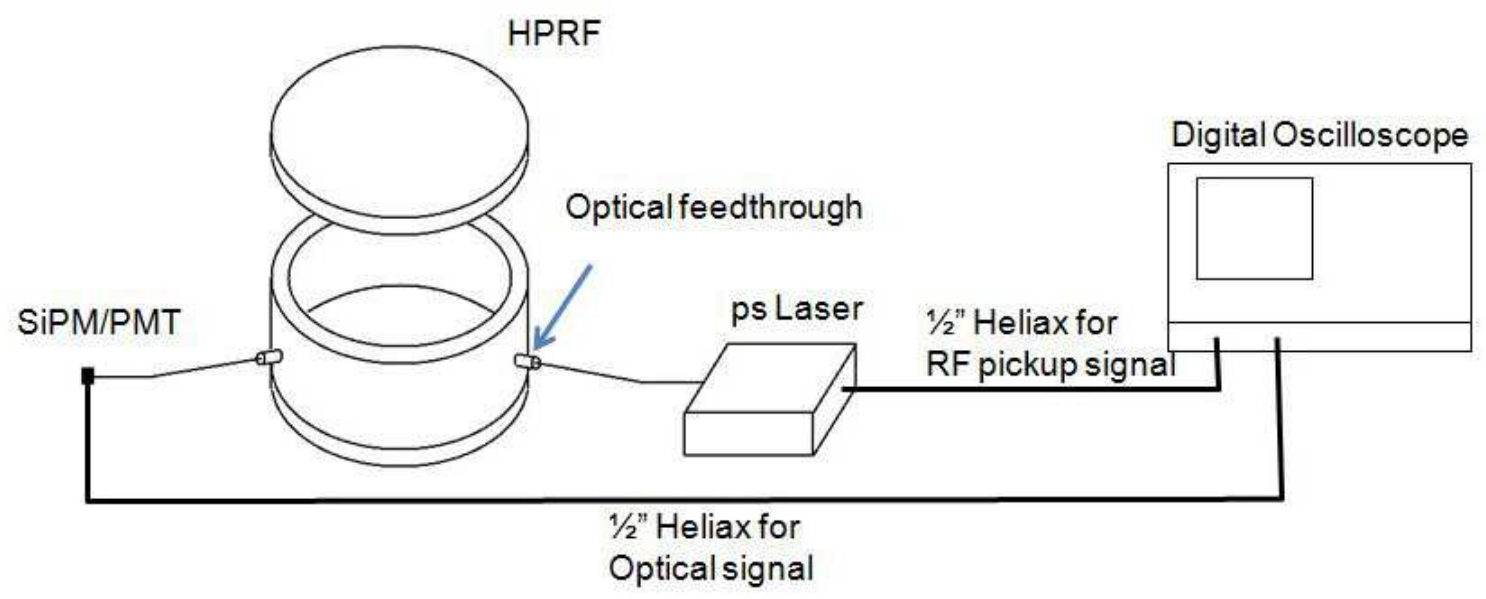

Figure 2.15. Schematic for the cable timing calibration.

2.3.6 PMTs and SiPMs. Photomultiplier tubes work on the principle of the photoelectric effect and electron multiplication [114]. When a photon strikes the 
Table 2.5. Signal cable assignment.

\begin{tabular}{ll}
\hline \hline Cable Designation & Instrument \\
\hline S1-01-L & Electric Pickup \\
S1-02-L & Magnetic Pickup \\
S1-01-S & Forward Power Cavity DC \\
S1-02-S & Reflected Power Cavity DC \\
S2-07-S & Upstream Toroid \\
S2-08-S & Downstream Toroid \\
S2-10-S & SiPM \\
S2-14-S & PMT \\
\hline
\end{tabular}

surface of a metal with a small work function (photocathode), an electron may be emitted (the photoelectric effect). If a potential difference is applied between the photocathode and another terminal (the dynode), the electron will accelerate toward the dynode and produce more electrons when it strikes it. If this process is repeated a number of times, an avalanche of secondary electrons can be produced. The result is a current proportional to the intensity of light incident on the detector.

Silicon photomultipliers are also used to measure the intensity of light, but use avalanche photodiodes (which also rely on the photoelectric effect, but not in a vacuum) on a substrate of silicon to count single photons [115]. SiPMs are advantageous to compared PMTs in that the gain is linear with supply voltage, as opposed to a power law, the supply voltage is much lower, and the time jitter is much smaller.

A great deal of light is produced in the cavity by the beam, from de-exciting 
Table 2.6. Signal cable delay.

\begin{tabular}{lc}
\hline \hline Cable Delay & Delay (ns) \\
\hline Magnetic pickup - Downstream toroid & $48.07 \pm 0.41$ \\
Magnetic pickup - Upstream toroid & $54.75 \pm 0.30$ \\
Magnetic pickup - Electric pickup & $3.27 \pm 0.35$ \\
\hline
\end{tabular}

hydrogen, recombining electrons and ions, etc. Two of the three optical ports in the cavity were used. A long optical fiber was employed to carry the light signal from the experimental apparatus further downstream in the hall to where the PMT and SiPM could operate outside of any magnetic field, be shielded from beam-induced radiation, and satisfy the 15 foot minimum distance requirement around the cavity due to hydrogen safety requirements. 


\subsubsection{Directional Couplers.}

There were a number of directional couplers (DC) at various places along the waveguide between the klystron and RF cavity. They served the purpose of measuring the power flowing into and out of the cavity. Figure 2.16 shows a diagram of a typical directional coupler.

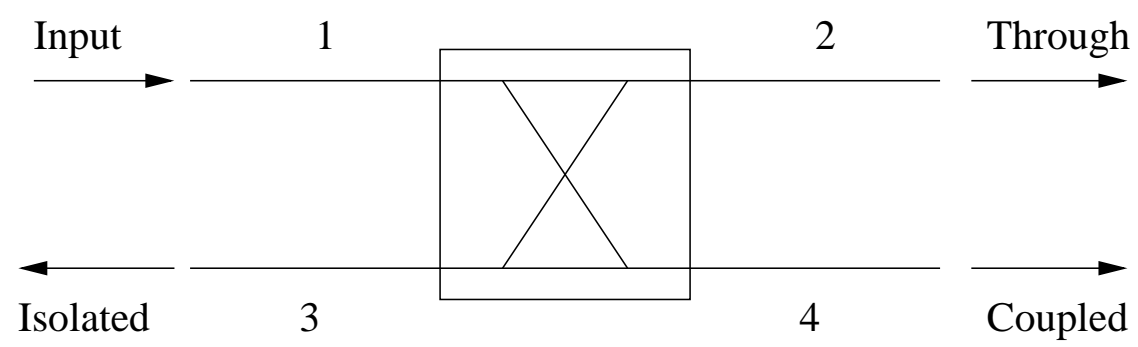

Figure 2.16. Schematic of a directional coupler.

Directional couplers have four term scattering matrices, similiar to that of a circulator. In Fig. 2.16, power is supplied to port 1 (input) and coupled to port 4 (" coupled") with a coupling factor $\left|S_{13}\right|^{2}=\beta^{2}[112]$. The remainder of the power goes to port 2 ("through"), with coefficient $\left|S_{12}\right|^{2}=\alpha^{2}=1-\beta^{2}$. For an ideal coupler, no power flows through port 3 ("isolated"). There are three values typically used to describe a DC: the coupling, $C$, which indicates the fraction of input power that goes to the output port; the isolation, $I$, which indicates how well the coupler isolates the forward and reflected waves; and the directivity, $D$, which is similar to the isolation, but with respect to the through port.

$$
\begin{aligned}
I & =10 \log \frac{P_{1}}{P_{3}}=-20 \log \beta \mathrm{dB} \\
C & =10 \log \frac{P_{1}}{P_{4}}=-20 \log \left|S_{14}\right| \mathrm{dB} \\
D & =10 \log \frac{P_{3}}{P_{4}}=20 \log \frac{\beta}{\left|S_{14}\right|} \mathrm{dB}
\end{aligned}
$$

Figure 2.17 shows the directional coupler closest to the cavity, used to measure the forward and reflected power during the experiment. One can see that the isolated port is terminated while the signal cable is connected to the coupled port. 


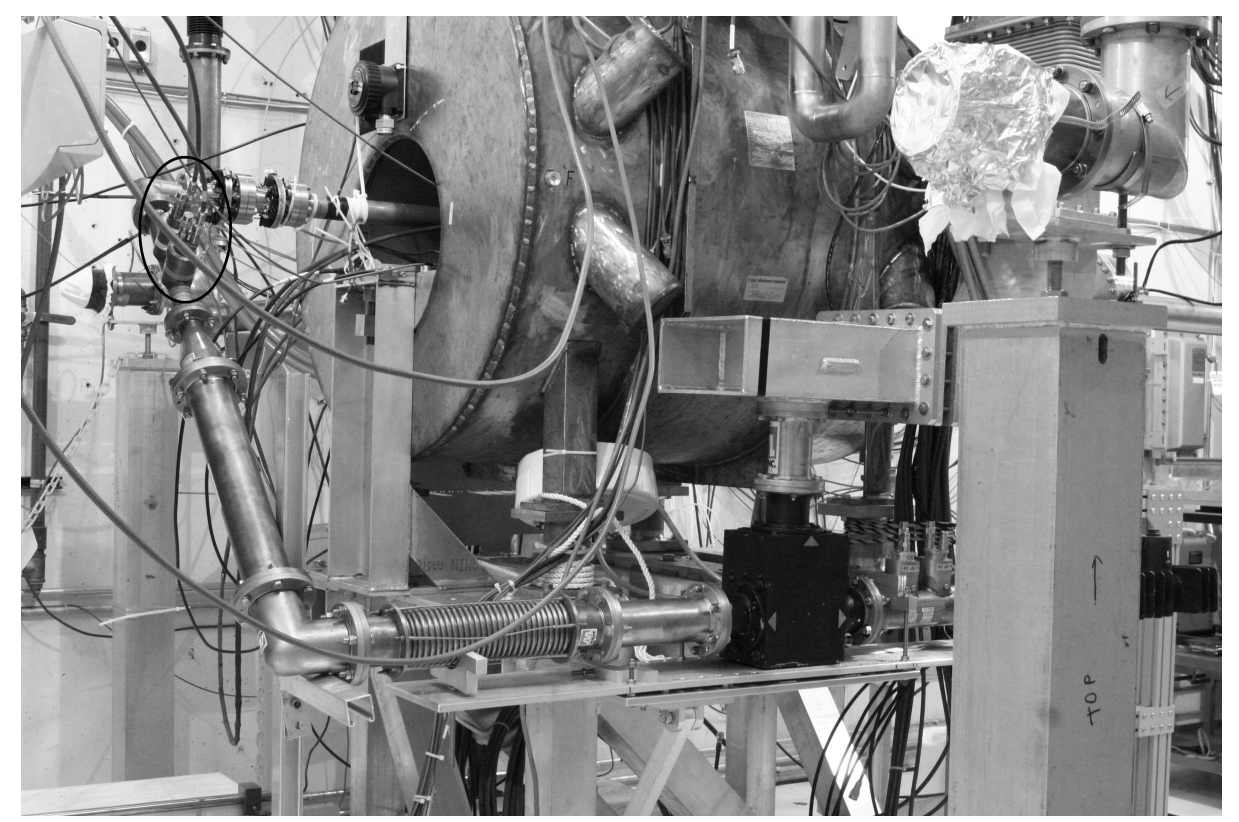

Figure 2.17. Directional coupler (circled, top left) closest to the HPRF cavity. The circulator and load are also visible in the bottom right.

2.3.8 Gas Handling System. Because of our requirements for working with pressurized gas and for hydrogen gas, a system to safely pressurize the cavity was implemented. To allow pressurizing and depressurizing the cavity without having to enter the MTA hall, a gas shed was built outside of the experimental enclosure. Figure 2.18 shows the layout of the gas shed. Gas bottles were hooked up to the gas handling system and stored there. Because hydrogen gas was stored there, every piece of equipment in the gas shed had to be explosion proof.

Figure 2.19 shows a diagram of the gas handling system. The cavity was filled and vented using two valves (TCFV and TCVV in Fig. 2.19, respectively) on a gas manifold that contained a pressure gauge calibrated to 3000 psi (TCGP). In addition to the vent line, there was a pressure relief line with a regulating valve calibrated to open at 1600 psi (TCRV).

The cavity was pressure tested to 1760 psi, with the maximum operating pressure limited to 1520 psi. Because hydrogen was used, no energized equipment could 


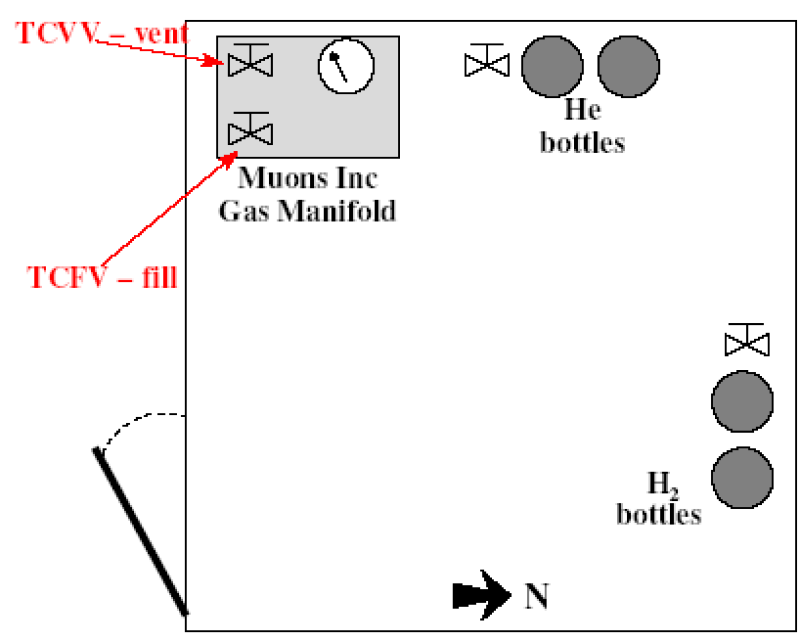

Figure 2.18. Layout of the gas shed [116]. The fill and vent valves (red) located on the gas manifold were used to pressurize and depressurize the cavity.

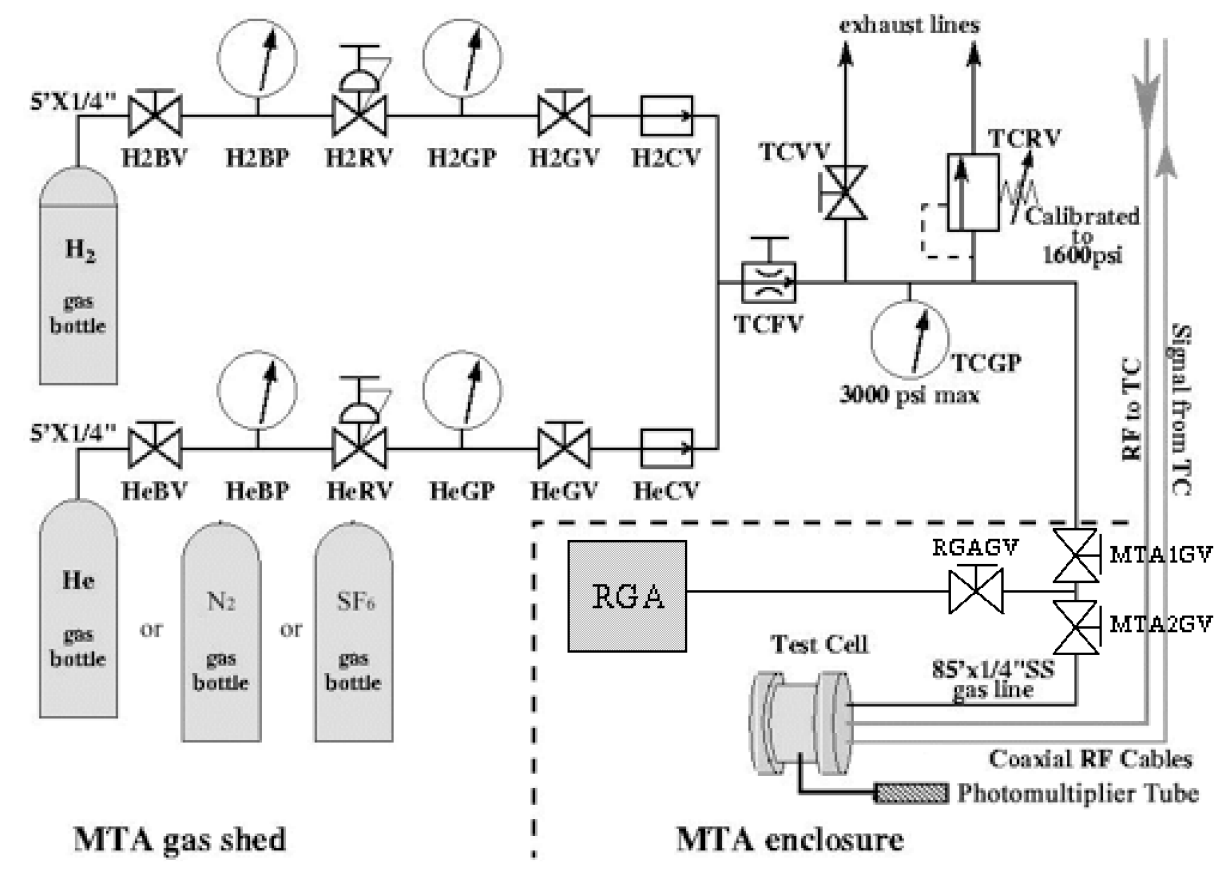

Figure 2.19. Schematic of the gas handling system [116]. There were 14 manual valves or regulators (labeled with a "V" at the end of the name) and five pressure gauges (labeled with a "P" at the end of the name). The residual gas analyzer (RGA) was not present in the experiment. 
be placed within $15 \mathrm{ft}$ of the cavity in the hall, or brought into the gas shed. The procedure for pressurizing the cavity was as follows. First, the cavity was purged with $\sim 140$ psi helium three times to remove any reactive gases from the cavity. If the run used pure hydrogen, the cavity was purged with $\sim 140$ psi hydrogen five times, to ensure hydrogen purity for the experiment. Research grade hydrogen was used (99.999\% purity). If the run used dry air-doped hydrogen, a different procedure was used: first, the cavity was purged with $\sim 140$ psi dry air three times to remove the helium. Then the cavity was filled with the desired partial pressure of dry air. Finally, the cavity was filled with hydrogen to reach the desired total pressure. At the end of the run, all gas was vented from the cavity, and the cavity was purged three times with $\sim 140$ psi helium gas to remove any residual hydrogen. The cavity was left at atmospheric pressure.

\subsection{Run Conditions}

Data were collected during two beam runs, the first from July 12 to August 8, 2011, and the second from April 13 to May 8, 2012. During this time a variety of gas species, gas pressures, electric fields, and beam intensities were investigated.

2.4.1 Run I. The primary purposes of Run I were to investigate the physics of the plasma formed in the cavity by the passage of beam through hydrogen and to study the effect of doping hydrogen with an electronegative gas. For this run hydrogen, nitrogen, hydrogen doped with nitrogen, and hydrogen doped with sulfur hexafluoride at pressures of 500, 800 and 950 psi were used. Safety requirements for the bolts used to assemble the cavity limited the pressure to 950 psi. The HPRF cavity was operated at 10, 20 and $30 \mathrm{MV} / \mathrm{m}$. The primary RF pulse length was $40 \mu \mathrm{s}$, with longer pulses of $80 \mu$ s occasionally recorded. The primary beam pulse length was $7.5 \mu \mathrm{s}$, with longer pulses of $35.5 \mu \mathrm{s}$ occasionally recorded. Three beam intensities, ranging from $\approx 4 \times 10^{10}$ to $1.5 \times 10^{12}$ protons/pulse (at $7.5 \mu \mathrm{s}$ ) incident on the HPRF 
cavity were used. The range of bunch intensities was $\approx 2.7 \times 10^{7}$ to $10^{9}$ protons $/$ bunch (at $7.5 \mu \mathrm{s}$ ). See Appendix A for a complete list of run conditions for Run I.

The data were recorded on a combination of three oscilloscopes, nicknamed "Electron," "Muon," and "Tau." The manufacturer of each was Tektronix and the model numbers were TDS-694C, DPO-7104 and TDS-5104, respectively. The scope with the best timing resolution was Muon, at $200 \mathrm{ps} /$ point, and that was used to record the magnetic pickup, downstream toroid, spectrometer, and PMT signals. The forward and reflected RF power from the two directional couplers, one near the cavity and one near the circulator load, were recorded on the Electron scope. Tau was set up to record the magnetic pickup signal, sent through an RF envelope detector, with a $66 \mathrm{~ms}$ delay with respect to the other two scopes. This allowed the next RF pulse (at a $15 \mathrm{~Hz}$ repetition rate) after the beam traversed the cavity to be recorded. Each scope was triggered with the timing signal from the Linac C-magnet that indicated beam was being sent to the MTA. When Muon and Electron received a trigger signal they recorded each trace of the scope to a comma-separated-values (csv) file along with a screenshot of the traces. When Tau received a trigger it would do the same after the set delay time. A video of the scintillating screen was also recorded, using a CCD camera.

2.4.2 Run II. The primary purposes of Run II were to expand the data set taken during Run I with improved instrumentation and data logging. Improvements in instrumentation included the shielding from particle collisions and new cores for the MTA toroids (during Run I there was no aluminum housing), a Silicon Photomultiplier (SiPM), and a repaired electric pickup probe. Data logging was controlled by a PC running LabVIEW, which received the same trigger signal as in Run I and then triggered each oscilloscope simultaneously [117]. The LabVIEW program was also used to tune the cavity to the resonant frequency and desired gradient. The cavity 
itself was outfitted with new bolts that allowed safe operation up to a pressure of 1520 psi. Additionally, some data were collected with the MTA magnet turned on.

For Run II, data were collected using the gases and pressures listed in Tab. 2.7. Various concentrations of dopants were used. The cavity was operated at 5, 10, 18, 20, 25, 30, 40, and $50 \mathrm{MV} / \mathrm{m}$ with a $40 \mu \mathrm{s}$ RF pulse length. The beam pulse length varied between 7.5 and $9.5 \mu \mathrm{s}$. Four distinct beam intensities were attempted, however due to the inability to stabilize the intensity at the lowest setting for any length of time, the majority of data were taken at three beam intensities. Unfortunately the highest beam intensity achieved during Run I was not reproducible. However it was possible to go lower in beam intensity, and so the intensity range was $\approx 10^{10}$ to $4 \times 10^{11}$ protons/pulse incident on the HPRF cavity. The range in bunch intensity was $\approx 5.3 \times 10^{6}$ to $2.1 \times 10^{8}$ protons $/$ bunch (at $9.5 \mu \mathrm{s}$ ). See Appendix B for a complete list of run conditions for Run II.

The upper flammability limit of oxygen gas in hydrogen gas is about 5\%. Two steps were taken to ensure that this limit was not exceeded. First, pure oxygen was never mixed directly with hydrogen. As shown in Tab. 2.7, dry air (DA) was used in the place of oxygen. Dry air is approximately $80 \%$ nitrogen and $20 \%$ oxygen. Second, the ratio of dry air to hydrogen was kept such that the safe allowable limit for oxygen was never approached.

A new LeCroy Waverunner 625Zi oscilloscope was purchased for Run II. It was capable of recording four channels of data at $50 \mathrm{ps} / \mathrm{pt}$ and was used as the primary scope. The electric and magnetic pickup, downstream toroid, and SiPM signals were recorded on the LeCroy scope. Two of the channels on Muon were not trustworthy and so it was used to record only the upstream toroid and PMT signals as it had the next best timing resolution. The Electron and Tau scopes could not interface with LabVIEW and so were used sparingly to record directional coupler signals. The 
Table 2.7. Gas Species and Pressures for Run II.

\begin{tabular}{ll}
\hline \hline Gas & Pressure (psi) \\
\hline hydrogen & $300,500,800,950,1100,1140,1300,1470,1520$ \\
deuterium & $500,800,1140$ \\
helium & 1470 \\
nitrogen & 500,700 \\
dry air & 800 \\
hydrogen + dry air & $300,735,800,1100,1385,1450,1470$ \\
hydrogen + sulfur hexafluoride & $300,1100,1450,1470$ \\
hydrogen + nitrogen & 1100 \\
deuterium + dry air & $300,800,1100,1470$ \\
helium + dry air & 1470 \\
nitrogen + dry air & 700 \\
\hline
\end{tabular}

scintillating screen-CCD camera system was also used to record the profile of each beam pulse. The hope was that this would be a precise enough system to allow for a beam intensity measurement when the magnet was energized and therefore the toroids did not work. Unfortunately the error in the measurement was too large to measure the beam intensity with the desired $(\sim 10 \%)$ precision (see Ref. [106]). 


\section{CHAPTER 3}

\section{RESULTS}

The experimental setup at the MTA does not allow one to measure the performance of an HPRF cavity under real muon cooling-channel conditions; the gas pressure and plasma density are both too small. What one is forced to do then is use the results obtained in these beam tests to extrapolate to the conditions in a real cooling-channel and estimate the performance based on the physics that can be ascertained.

The main question one must answer is, how much plasma loading can be expected when $5 \mathrm{~ns}$ spaced bunches of $10^{12}$ muons pass through a cavity with up to $180 \mathrm{~atm}$ of hydrogen gas? This can be broken up into three separate measurements: how many charged particles are produced, how much energy each absorbs over an RF cycle, and how long they exist inside the cavity. The number of electron-ion pairs produced is given by Eq. 1.21, where all that is needed is an accurate measurement of the gas density and beam current. The amount of energy an electron or ion absorbs can be modeled by Eq. 1.28, and measured by taking the power absorbed by the plasma and dividing by the number of particle-cycles. Finally, the lifetime of an electron or ion can be calculated through the previous energy loss calculation and the measured power deposited in the plasma as a function of time.

Unless otherwise stated, all analysis was done using Mathematica [118]. Approximately 2500 beam pulses were recorded using hydrogen and dry air-doped hydrogen during the course of the experiment. Signals from the two RF pickups, downstream toroid, and SiPM were sampled every $50 \mathrm{ps}$ for $50 \mu \mathrm{s}$, (for a total of $10^{6}$ points). The signals from the upstream toroid, PMT, and directional couplers were recorded at $200 \mathrm{ps} / \mathrm{pt}$. 


\subsection{Energy Loss}

A reduced data set was produced consisting of: RF amplitude envelope and its derivative, beam current, integrated beam current, power fed to the gas by the klystron, power fed to the gas by the cavity, total power fed to the gas, and energy loss. The reduced data were produced having been averaged over $10.05 \mathrm{~ns}$. This was done mainly to reduce noise, but also to reduce the data volume.

To obtain the amplitude envelope from the raw RF signal, the data was fit with a function of the form $E(t)=c_{0}+c_{1} \cos (\omega t)+c_{2} \sin (\omega t)$. The average (center)

time of the segment was then recorded along with the amplitude, $\sqrt{c_{1}^{2}+c_{2}^{2}}$ and the offset, $c_{0}$.

Figure 3.1 shows a typical plot of the amplitude of the electric field in the cavity vs. time. The plot begins once the electric field amplitude has reached its flat top. At $1 \mu$ s the beam turns on, and the electric field drops to a minimum ("equilibrium") at $5 \mu \mathrm{s}$. The beam turns off at $9 \mu \mathrm{s}$, at which time the electric field in the cavity begins to rise again. The RF power input is turned off at $26 \mu \mathrm{s}$, and the electric field in the cavity decays.

The equivalent circuit for a beam and plasma loaded cavity is shown in Fig. 3.2. The generator (in our case a klystron) sends RF power down a matched transmission line, where it is inductively coupled to the cavity. The cavity acts as an RLC circuit with shunt impedance, $R_{c}$. The gas is a source of energy loss and therefore acts as resistive component. The momentum spread is 0.005 at the end of the Linac and the bunch length of the beam becomes larger during the roughly $45 \mathrm{~m}$ without $\mathrm{RF}$ to the experimental apparatus. The beam arrives at a random phase with respect to the RF wave, and the cavity is driven at a frequency that is not a harmonic of the Linac frequency, and so the beam loading is negligible (as much energy is given to 


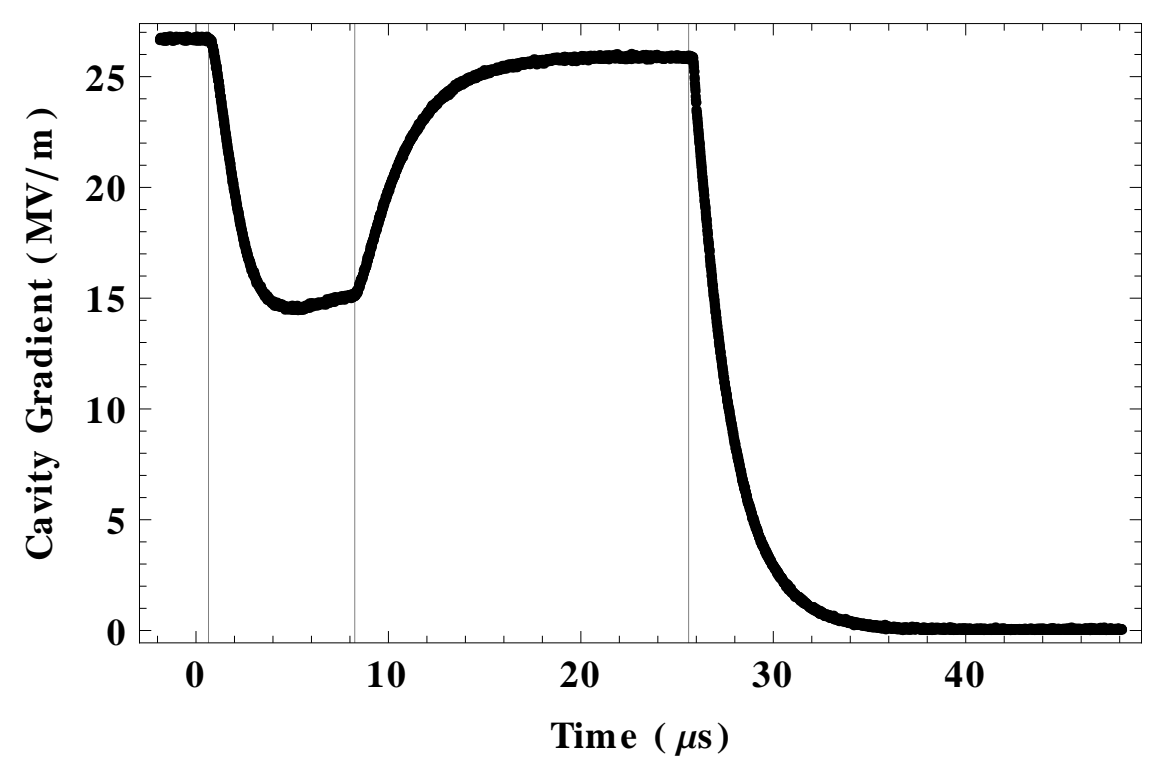

Figure 3.1. A typical RF envelope. The amplitude of the RF electric field is plotted vs. time.

the cavity as taken).

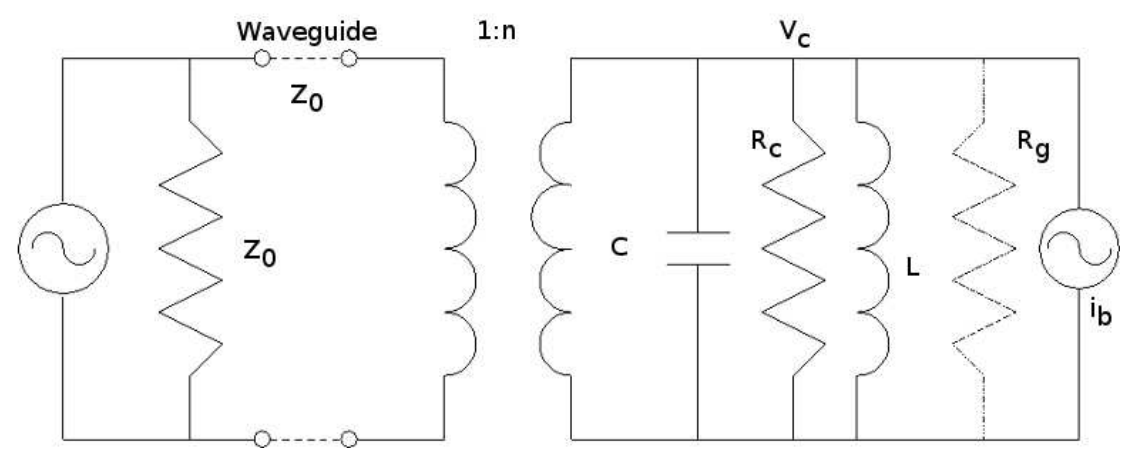

Figure 3.2. The equivalent circuit of a plasma and beam loaded cavity.

The power being delivered to the gas is given by:

$$
P=\frac{\left(V_{0}-V\right) V}{R_{c}}-C V \frac{d V}{d t}
$$

where $V_{0}$ is the flat top voltage and $V$ is the voltage at time $t$. The voltage is found by using the average electric field across the accelerating gap. The first term is the power provided by the klystron, and the second term is the power provided by the cavity (coming from its stored energy). 
The capacitance of the cavity has been simulated using SuperFish [119]. With 1 atm hydrogen in the cavity and $1 \mathrm{MV} / \mathrm{m}$ average electric field across the accelerating gap, the calculated resonant frequency is $810.03 \mathrm{MHz}$ and stored energy is $0.2338 \mathrm{~mJ}$. This corresponds to a capacitance of $1.48 \mathrm{pF}$ and an inductance of $26.08 \mathrm{nH}$. The capacitance (and therefore frequency) will change with gas pressure, while the inductance will not. We will use the calculated inductance of the cavity to find the capacitance as a function of the measured resonant frequency.

To find the resistance of the cavity, the exponential decay of the RF envelope was fit, and the resistance calculated based on the time constant and the capacitance, $E(t)=E_{0} e^{-t / \tau}, \tau=R C$. This measurement was made on RF pulses without beam so as to obtain the unloaded resistance. Figure 3.3 shows a typical fit to the decay of the cavity gradient once RF power is turned off. The resistance for each combination of gas pressure and species and electric field was measured in this way. A table of the fitted time constants is given in App. C.

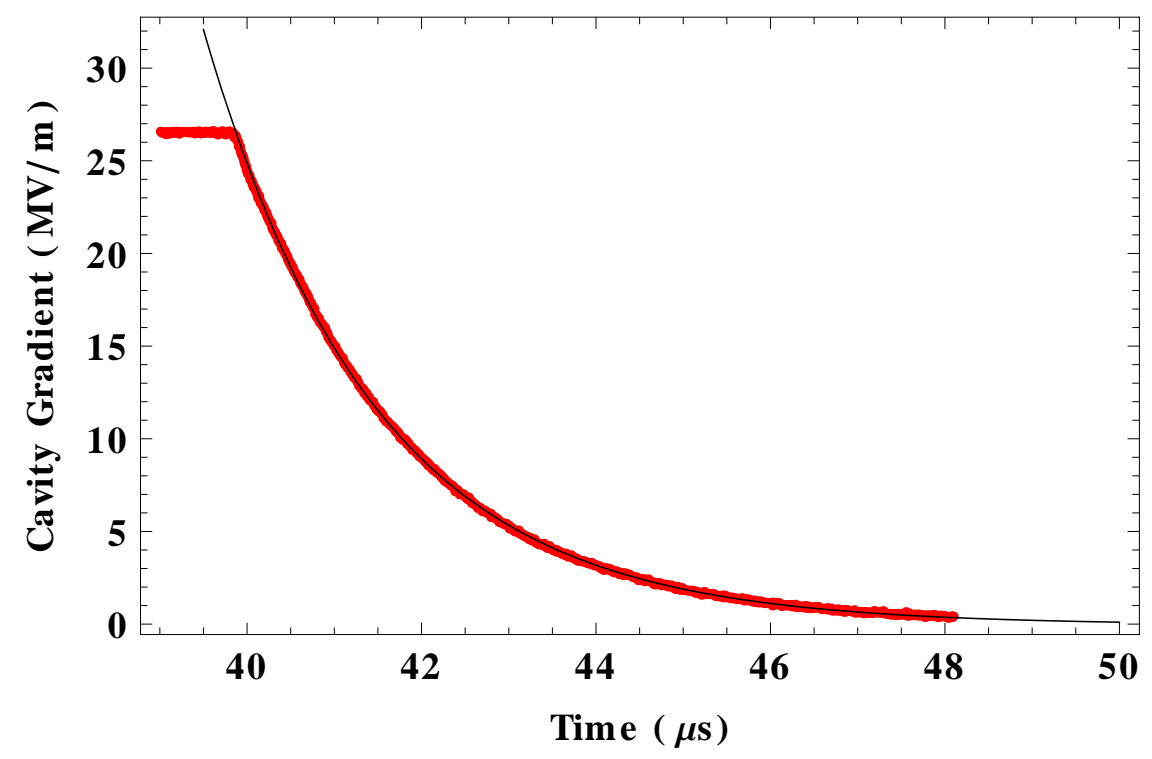

Figure 3.3. A typical cavity gradient decay fit. The envelope of the gradient is in red and the fit to the decay is in black.

The time derivative of the RF envelope, along with the RF envelope and 
calculated resistance and capacitance were used with Eq.3.1 to produce the reduced data for gas, klystron, and cavity power as a function of time. These data were also averaged over $10.05 \mathrm{~ns}$. It is important to note that the calibration of the pickup probe represents the peak electric field at the tip of the electrodes, with a distribution shown in Fig. 2.10. Because the plasma occupies a volume inside the cavity, it sees an electric field distribution. The average field on axis is $E_{a v g}=0.826 E_{\text {peak }}$ and this will be used in the power calculation. The assumption is also made that the plasma does not distort the field distribution in the cavity.

The beam current is found using Eq. 2.8. First, a section of the toroid signal before the beam turns on is fit and any offset is subtracted from the signal. Then the current as a function of time is calculated from:

$$
I(t)=\frac{N}{R}\left(V(t)+\frac{R}{L} \Delta t \sum_{\mathrm{t}=0}^{t} V(\mathrm{t})\right)
$$

where $N=5$ turns, $R=50 \Omega, \Delta t=50$ ps (the sampling rate was 20 gigasamples $/ \mathrm{s}$ ), and $L$ is the total inductance of the toroid system. The beam current is then corrected for the cable attenuation as given by Eq. 2.12 and averaged over 10.05 ns. The running total number of protons incident on the cavity as a function of time within the RF pulse is given by:

$$
N_{p}(t)=\frac{\Delta t}{e} \sum_{\mathrm{t}=0}^{t} I(\mathrm{t})
$$

where $e$ is the charge of a proton. This results in plots such as Fig. 3.4. In order to correlate the beam intensity with the corresponding power at a given time, the time at which the beam turned on is important. To find this, the first $60-70$ ns of Fig. 3.4, after a minimum intensity $\left(2 \times 10^{7}\right.$ protons $)$ is reached, is fit with a linear function and extrapolated back to $N_{p}=0$. This is defined as $t_{0}$ for this beam pulse. The number of protons is then averaged over $10.05 \mathrm{~ns}$. In order to use the correct relative timing, the measured cable delays (see Tab. 2.6) in addition to the $1.4 \mathrm{~ns}$ time of flight between the downstream toroid and the cavity are used for the appropriate signals. 


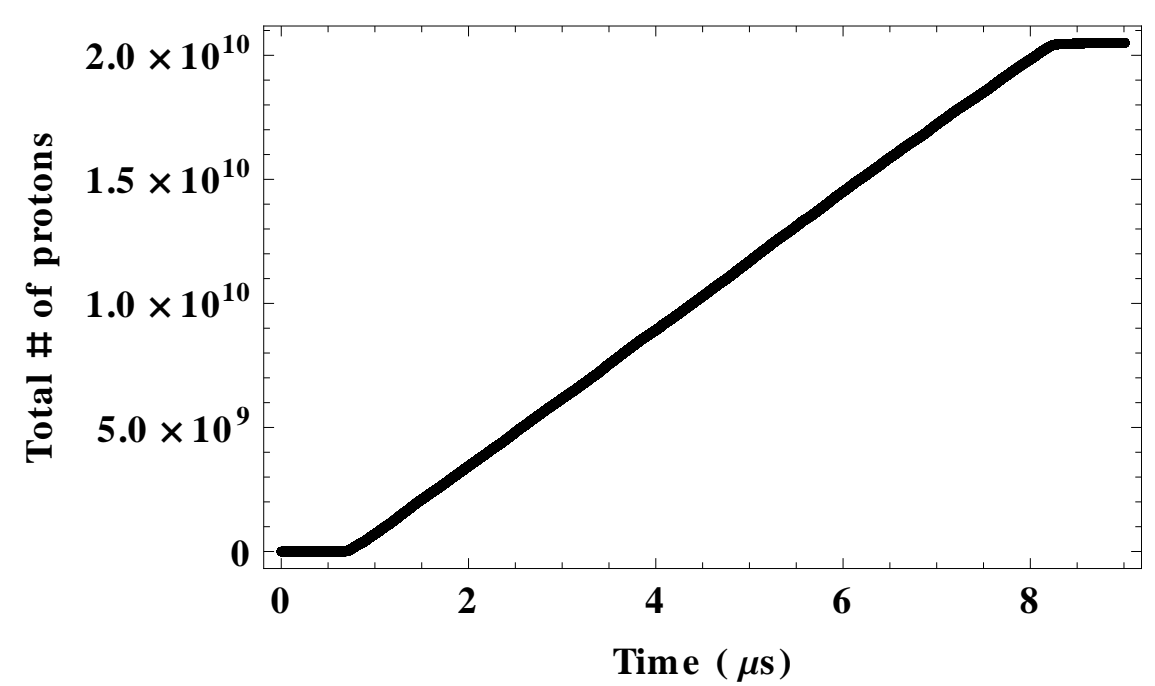

Figure 3.4. A typical beam intensity plot. The integrated number of protons incident on the cavity vs. time is shown. The total intensity of this pulse was $\approx 2 \times$ $10^{10}$ protons.

To calculate the number of electron-ion pairs produced per incident proton, Eq. 1.21 is used. For the case of hydrogen, $\frac{d E}{d x}=6.33 \frac{\mathrm{MeV} \mathrm{cm}^{2}}{\mathrm{~g}}, W_{i}=35.3 \mathrm{eV}$, and the density ranges from 1.71 to $8.67 \times 10^{-3} \frac{\mathrm{g}}{\mathrm{cm}^{3}}$ (for $300-1520 \mathrm{psi}$ ). Because hydrogen is not an ideal gas, a correction to the density is made based on the Van der Waals equation, which for our data set corresponds to $\rho_{V d W}=0.986-0.928 \rho_{\text {ideal }}$ (for $300-1520 \mathrm{psi}$ ) [119]. The $0.5 \%$ momentum spread of the beam implies a $0.25 \%$ change in $\frac{d E}{d x}$, which was ignored. The length of the accelerating gap is $1.77 \mathrm{~cm}$. Ionization electrons can also ionize hydrogen. This has been simulated using G4beamline [120], and increases the number of electrons to 1.184 times that made by the proton beam itself [111]. Finally, electron-ion pairs are produced at a rate of about $630-3000$ pairs/proton.

As is the case with all measurements of the physics within the cavity, we can only measure the effect of the ensemble, and cannot distinguish between electron and ion. What we can do is measure the total energy loss in a given time, and normalize it by the number of pairs and length of time they have existed, or number of pair-cycles over that time. This will be the measurement of energy loss that we can compare to 
Eq. 1.28.

$$
d w_{\text {meas }}(t)=\sum_{\mathrm{t}=t_{0}}^{t} \frac{P_{\text {gas }}(\mathrm{t})}{N_{\text {pairs }}(\mathrm{t}) f}
$$

Figures 3.5 through 3.7 illustrate the method by which $d w$ is calculated. The shaded areas of Fig. 3.6 and 3.7 are summed over time using Eq. 3.4 to calculate $d w$. Figure 3.5 is used to produce Fig. 3.6.

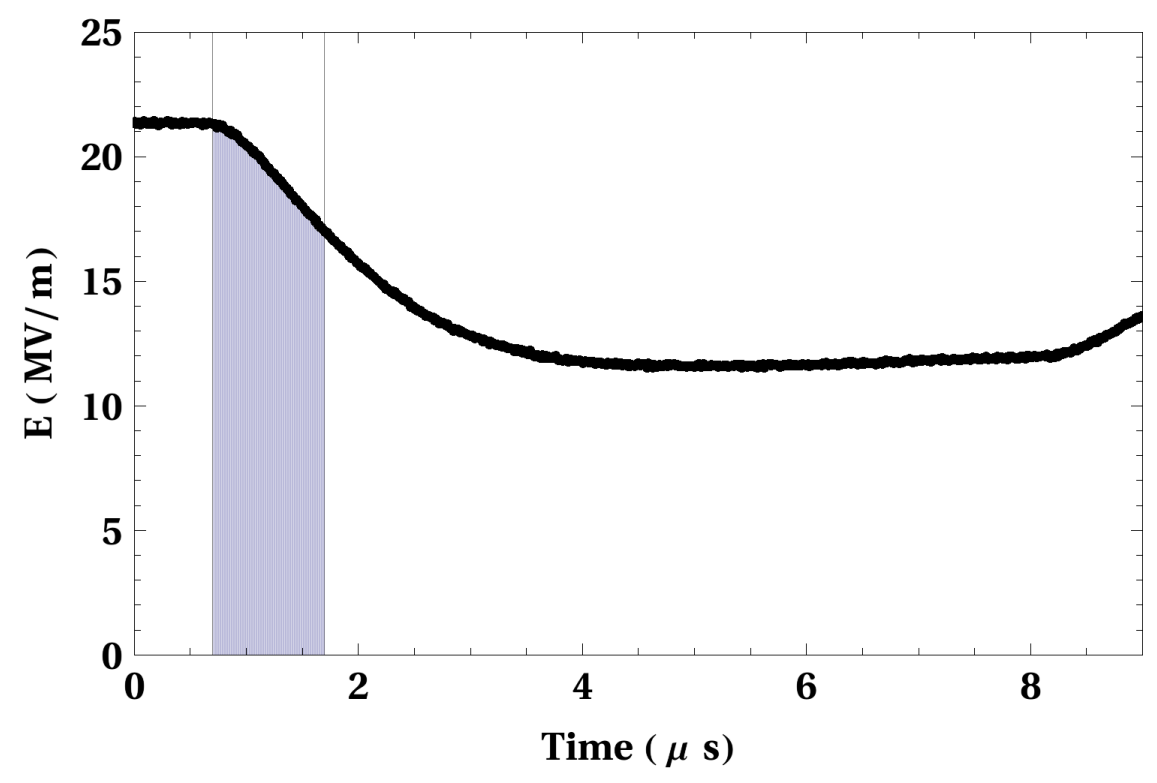

Figure 3.5. Example of the electric field used for a $d w$ calculation. The shaded area represents the time over which the calculation is made.

The total energy absorbed by the plasma will be found by integrating the gas power (Eq.3.1) over a given time. However, the number of charged particles in the cavity is changing with time (because each beam bunch produces new particles, and others are eventually lost to recombination or attachment). Once recombination begins $N_{\text {pairs }}$ (Eq. 1.21) is no longer an accurate measurement of the number of charged particles that have contributed to the energy loss. So for pure hydrogen we must confine ourselves to the very earliest part of the beam, during which time recombination is minimal. When hydrogen is doped with dry air, $N_{\text {pairs }}$ becomes the number of hydrogen ions and either electrons or oxygen ions. The resulting $d w$ measurement represents the sum of contributions from those three. 


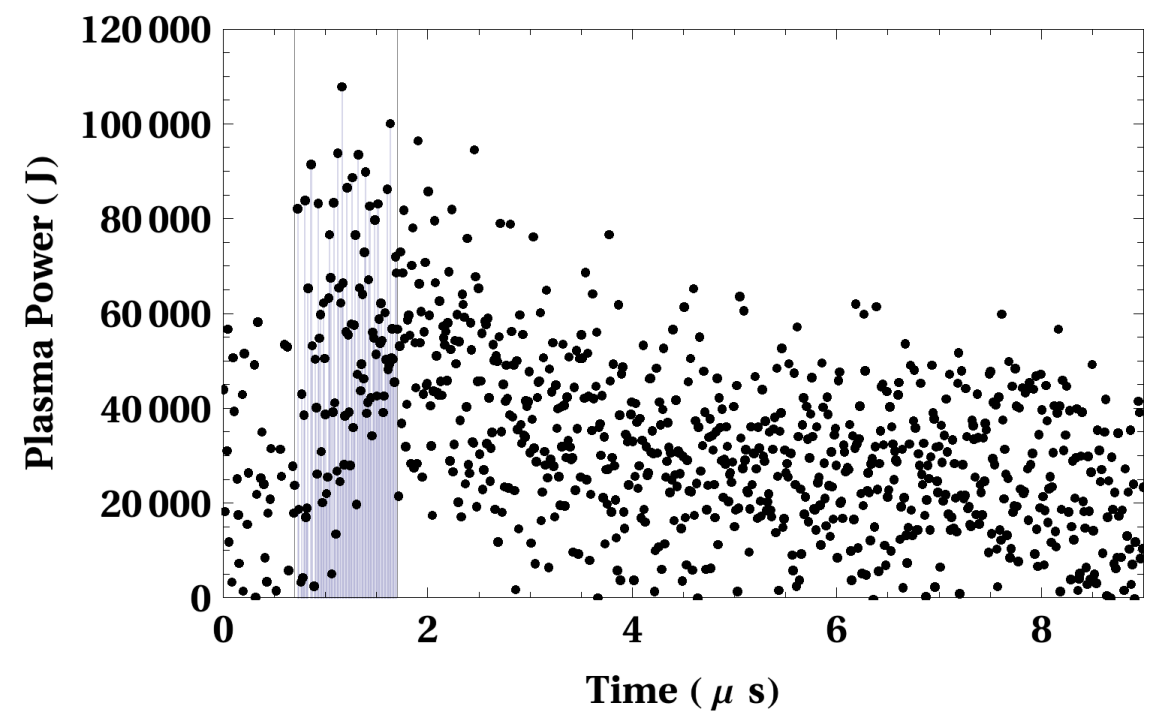

Figure 3.6. Example of the power used for a $d w$ calculation. The shaded area represents the time to be summed over.

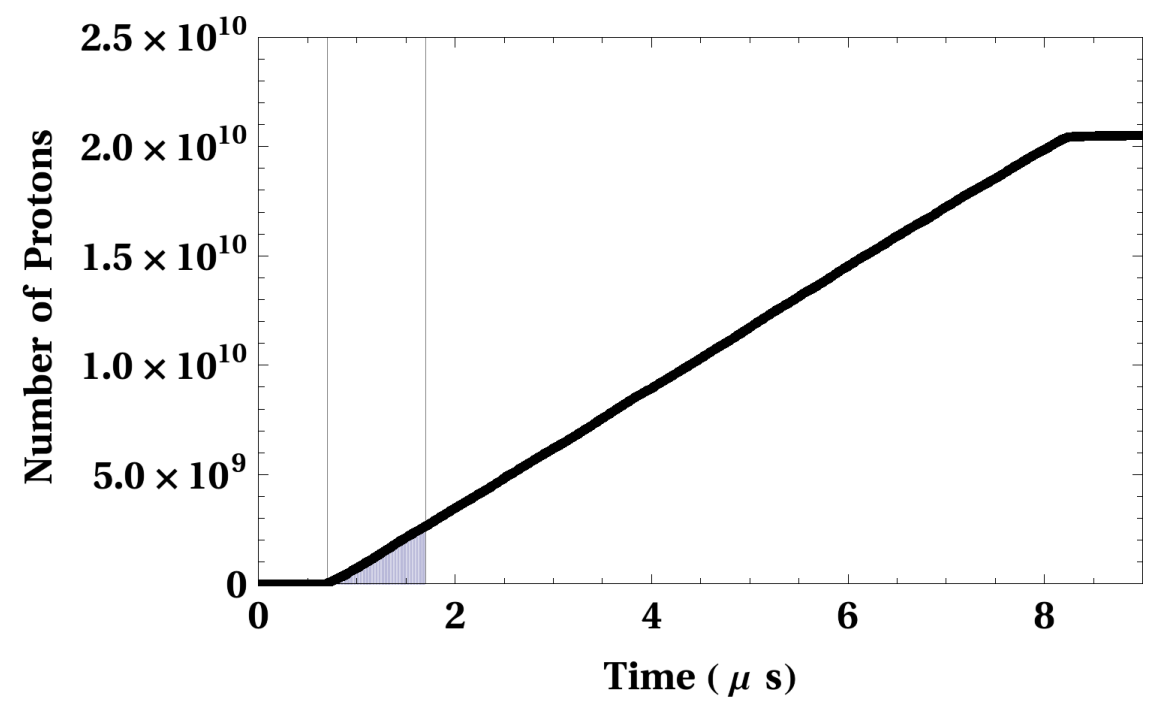

Figure 3.7. Example of the integrated number of protons used for a $d w$ calculation. The shaded area represents the time to be summed over. 
A simulation of the plasma distribution within the cavity has been performed, and will be described later. For now, we assume the plasma is confined to a cylinder with the diameter of the downstream collimator's through-hole $(4 \mathrm{~mm})$ and length equal to that of the accelerating gap $(1.77 \mathrm{~cm})$. This gives a volume of $0.222 \mathrm{~cm}^{3}$. The lowest plasma density will be that of the smallest beam intensity and gas pressure (about $2 \times 10^{10}$ protons/pulse and $300 \mathrm{psi}$ ). For a beam $7.5 \mu \mathrm{s}$ in length with $5 \mathrm{~ns}$ bunch spacing, this corresponds to 1500 bunches/pulse, or $\approx 1.3 \times 10^{7}$ protons/bunch, or $8.4 \times 10^{9}$ pairs/bunch. If we consider the first $100 \mathrm{~ns}$ of the beam (20 bunches), that's $1.7 \times 10^{11}$ pairs, or $7.6 \times 10^{11} \frac{\text { pairs }}{\mathrm{cm}^{3}}$. We have seen in Sec. 1.2 .5 that the recombination rate can be as high as $\sim 10^{-6} \frac{\mathrm{cm}^{3}}{\mathrm{~s}}$. Assuming this recombination rate, we get $7.6 \times$ $10^{5}$ pairs $/ \mathrm{s}$, or a characteristic time of $1.3 \mu \mathrm{s}$, much longer than our measurement window. For the other extreme case of high beam intensity and gas pressure $(4 \times$ $10^{11}$ protons/pulse and $1520 \mathrm{psi}$ ), consider the first five bunches (25 ns). Assuming the same recombination rate, this gives a characteristic time of $55 \mathrm{~ns}$, again longer than our measurement window. So the higher in beam intensity and gas pressure we go, the shorter the amount of time we have in which to measure the energy loss.

To stay away from noise in the power or beam measurements associated with the beam turning on, measurements of the energy loss were made when the voltage in the cavity fell to $96 \%$ of its flat top value after the beam turned on. For higher beam intensities, this occurred earlier than for lower beam intensities. Figure 3.8 shows the beam intensities for all hydrogen data. Figure 3.9 shows the length of time it took for the voltage to fall to $96 \%$ vs. pressure. This plot indicates that our measurements are of the energy loss before recombination begins.

This procedure produced a $d w$ at the corresponding value of $X$ at which the electric field is $96 \%$ of its initial value for every beam pulse. From this point we corrected for variations in beam intensity and $X$ and then averaged the data over sets 


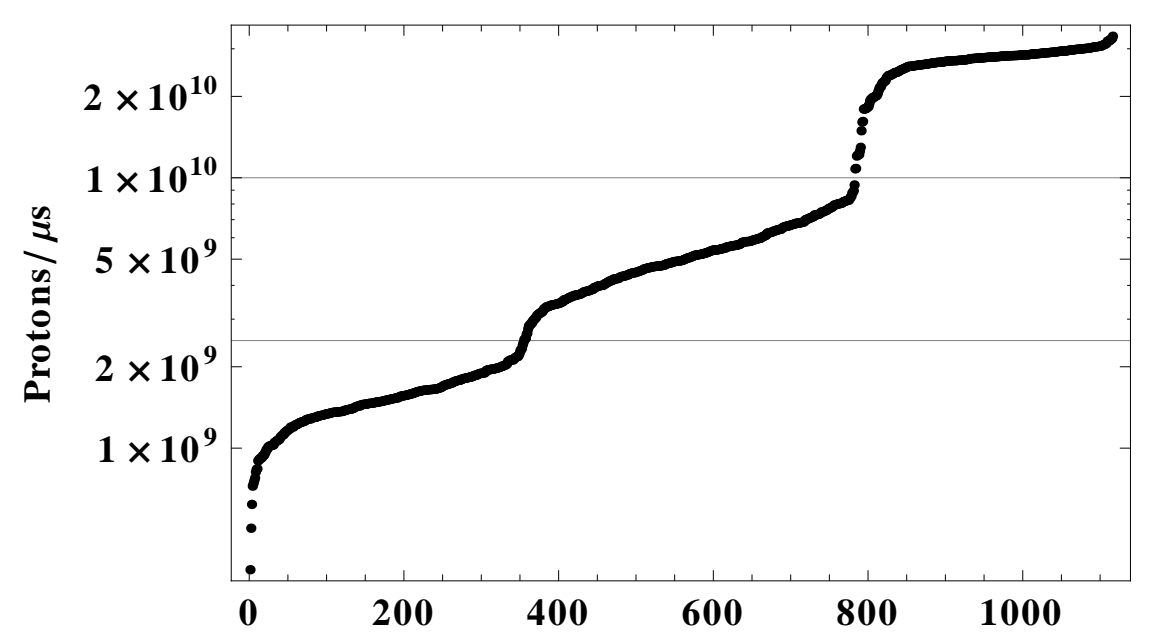

Figure 3.8. Beam intensities (in order of ascending magnitude) for all hydrogen data. The horizontal lines separate beam intensity settings, corresponding to full, $\approx 1 / 5$ and $1 / 10-1 / 20$.

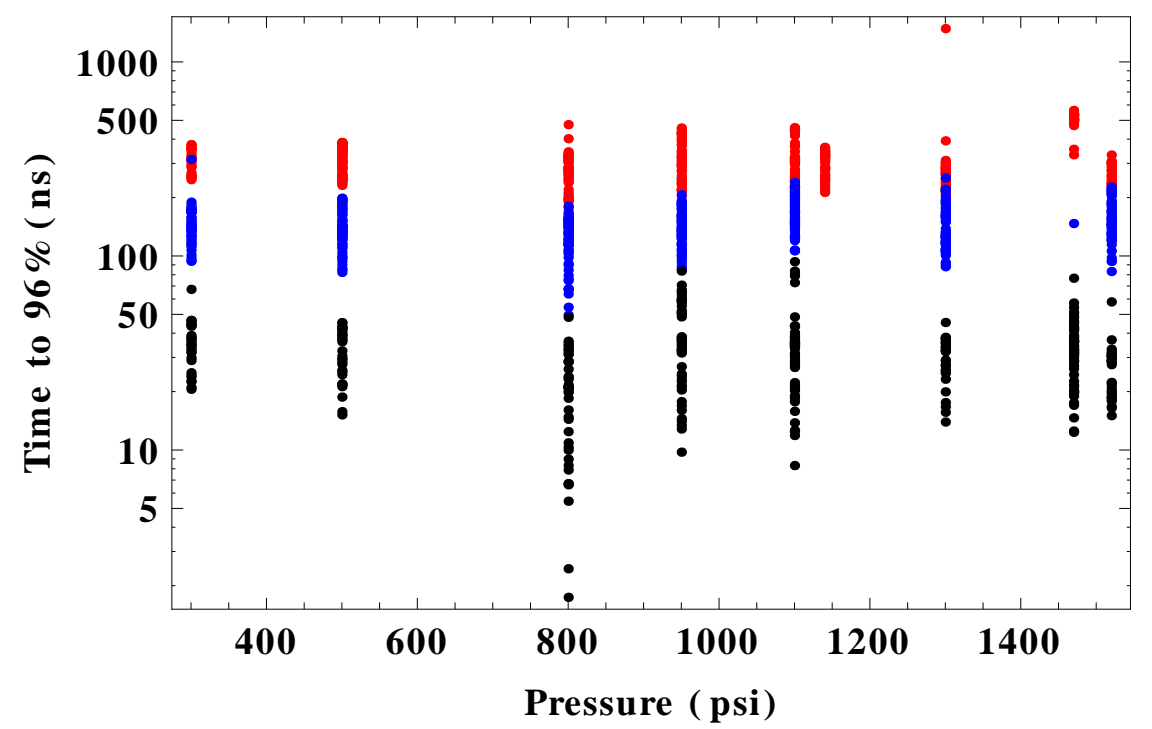

Figure 3.9. The length of time it took for the voltage to fall to $96 \%$ of the flat top value vs. gas pressure for pure hydrogen. Three beam intensities are shown, full (black), 1/5 (blue), and 1/10 - 1/20 (red). 


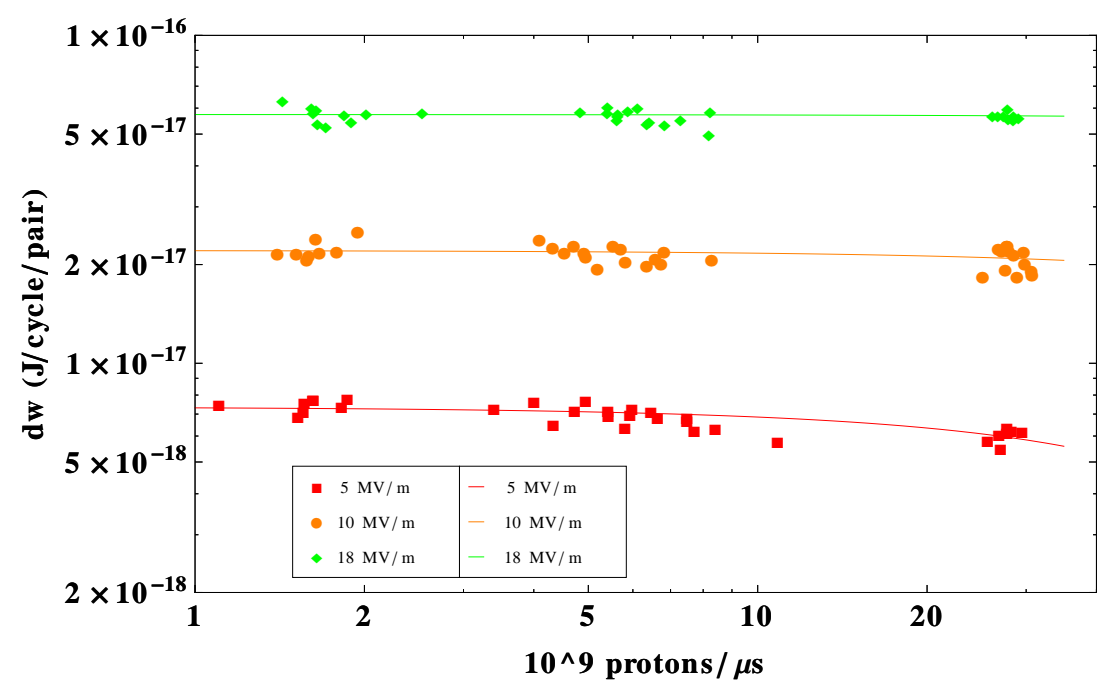

Figure 3.10. Example $d w$ vs. beam intensity fit at 300 psi.

of electric field and pressure. There was no overall beam intensity dependence for $d w$, however at a given electric field and pressure, $d w$ may have increased or decreased slightly (typically no more than 10\% over the full range of beam intensities) with increasing beam intensity. For each combination of $E$ and $P$ settings, a linear fit was made for $d w$ vs. beam intensity. The results of the fits for all of the 300 psi data are show in Fig. 3.10 as an example. Using the fits for each $E$ and $P$, the value of each $d w$ was extrapolated to the average of the highest beam intensity range for that set of data.

A similar procedure is used to correct for variations in the electric field. The set of all $(X, d w)$ at a given pressure is fit with a function of the form $d w(X)=c_{1} X^{c_{2}}$. The result for the 300 psi data set is shown in Fig. 3.11. The fit is used to shift all data points for a given electric field to the average value of $X$ for those measurements. This is done for each set of $E$ and $P$, and the corresponding values of $d w$ are then averaged. This results in an average data set in which groups of measurements at all beam intensities and similar electric fields have the corresponding values of $d w$ shifted to a central beam intensity and $X$. 


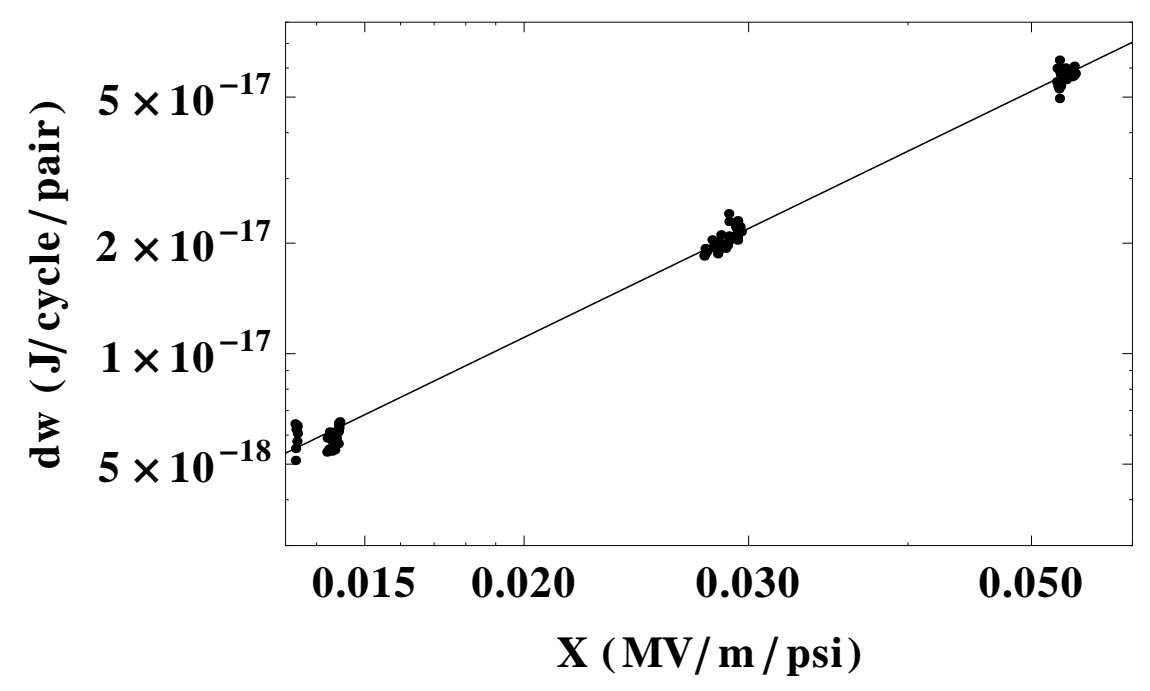

Figure 3.11. Example $d w$ vs. $X$ fit for $d w$ at 300 psi.

We will use the drift velocity of electrons in hydrogen gas at $300 \mathrm{~K}$ from Fig. 1.21 along with Eq. 1.28 as our model for predicting the energy loss in the HPRF cavity. The plasma distribution in the cavity has been simulated [111]. Due to the plasma and electric field distributions in the cavity, the energy loss we measure cannot be directly compared to that obtained from the drift velocity from [47] and Eq. 1.28. Rather, $d w_{\text {meas }}=0.66 d w_{e}=0.58 d w_{I}$, where the subscript $e$ stands for electrons and $I$ for ions, which is found by integrating the electric field distribution over the volume of the cavity.

Figures 3.12 and 3.13 show our data over the range of hydrogen pressures measured, with a dashed line that is a fit to the data, and a solid line that is the prediction of our model. Figure 3.14 shows the entire data set in one plot.

One can see that for low pressure the model fits the data very well. The data also match the prediction better at large $X$. For small $X$ and high pressure, the measured energy loss is lower than predicted (at $1520 \mathrm{psi}$ and $5 \mathrm{MV} / \mathrm{m}$, it is $73 \%$ of the predicted value). The pressure range over which the drift velocity of electrons in hydrogen used to make the prediction was measured is less than 10 psi [47]. 


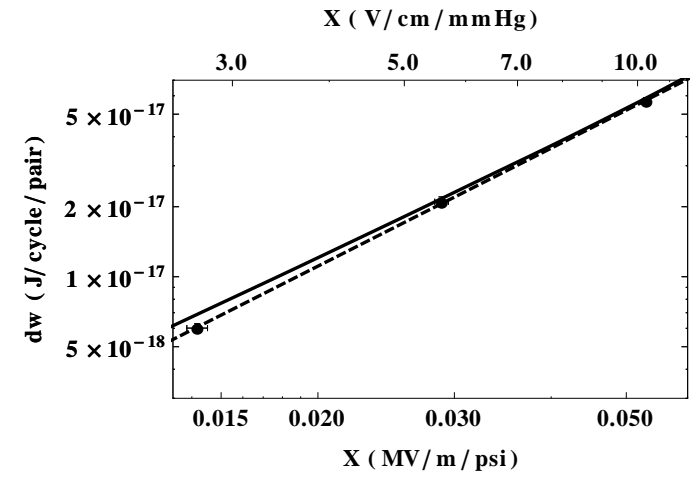

(a). $300 \mathrm{psi}$

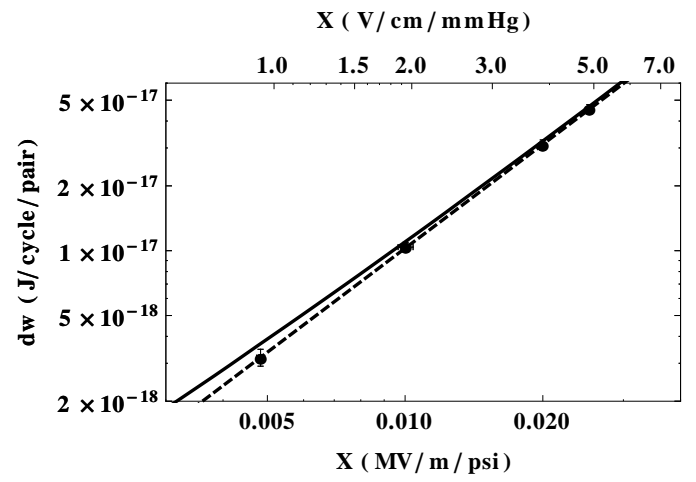

(c). $800 \mathrm{psi}$

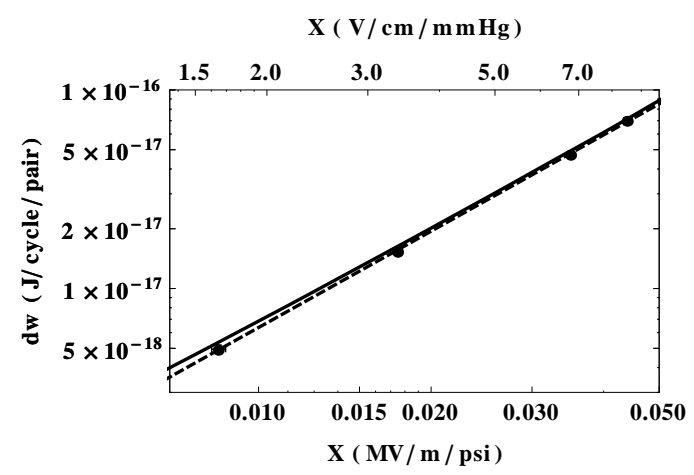

(b). $500 \mathrm{psi}$

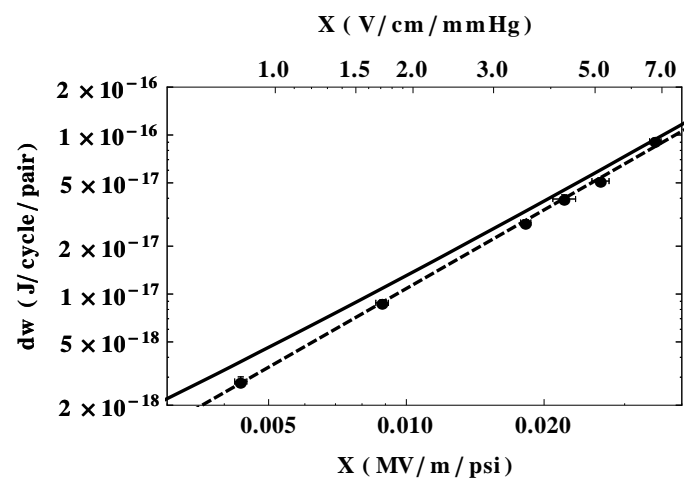

(d). $950 \mathrm{psi}$

Figure 3.12. $d w$ for $300-950$ psi hydrogen. The dashed lines are fits to the data, solid lines are predictions based on our model. 


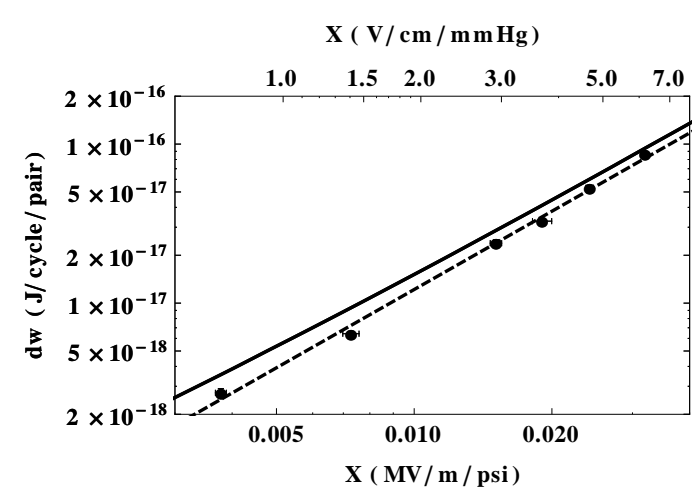

(a). $1100 \mathrm{psi}$

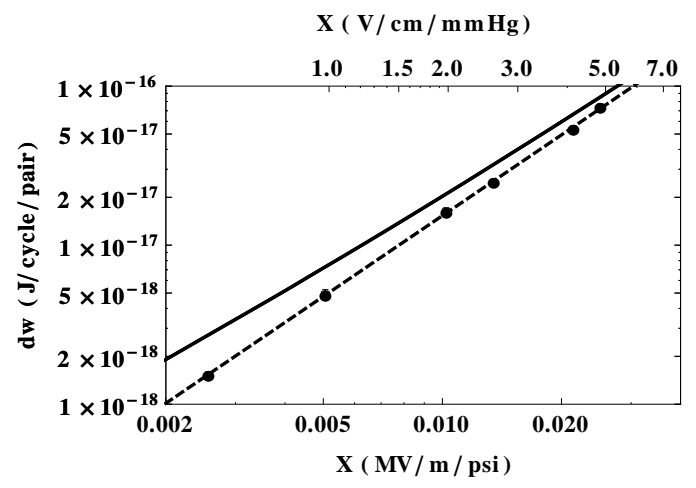

(c). $1470 \mathrm{psi}$

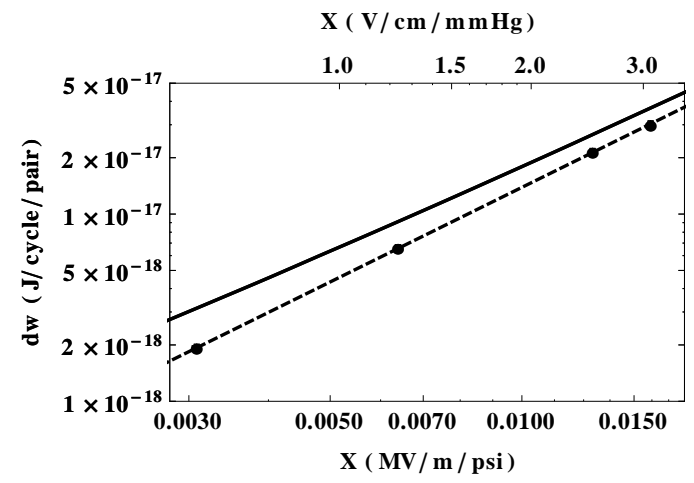

(b). $1300 \mathrm{psi}$

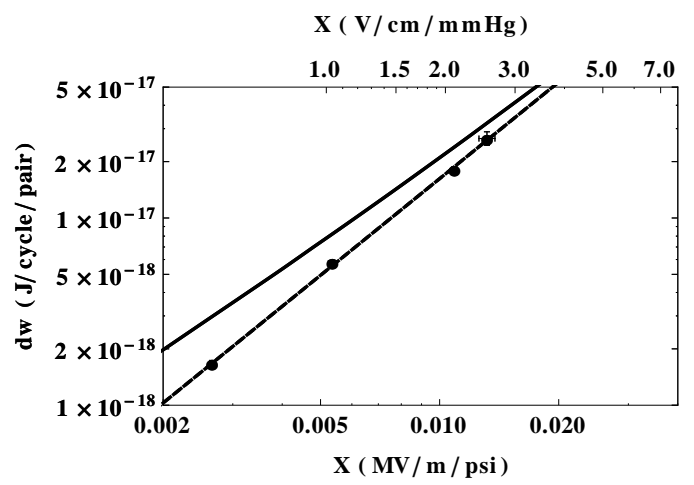

(d). $1520 \mathrm{psi}$

Figure 3.13. $d w$ for $1100-1520$ psi hydrogen. The dashed lines are fits to the data, solid lines are predictions based on our model. 


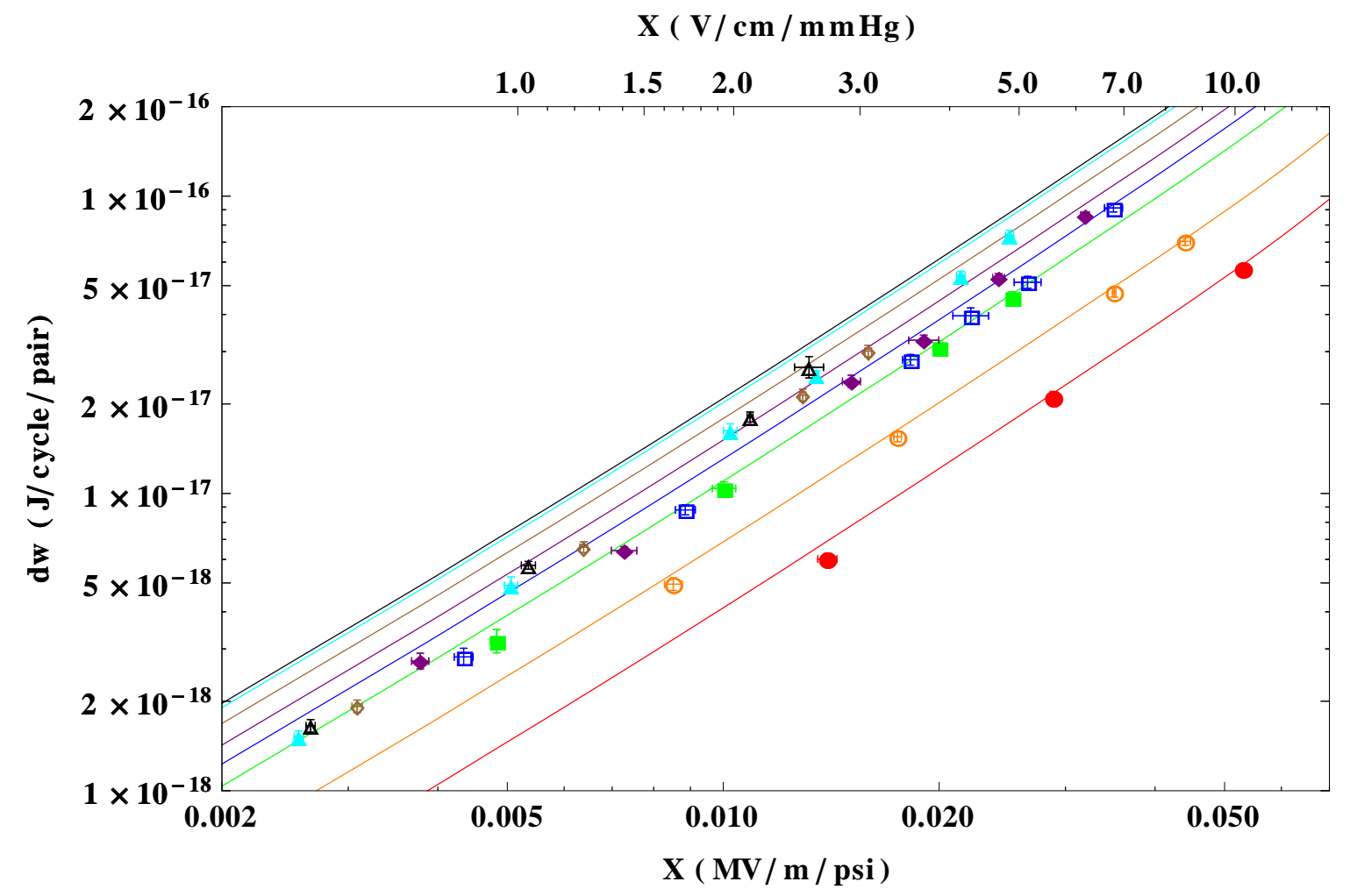

Figure 3.14. $d w$ for all pressures of hydrogen: 300 psi (red), 500 psi (orange), 800 psi (green), 950 psi (blue), 1100 psi (purple), 1300 psi (brown), 1470 psi (cyan), and 1520 psi (black). The solid lines are the model predictions. 
There have been a number of measurements $[54,55,121]$ indicating that the mobility (and hence drift velocity) of electrons decreases greatly with increasing gas pressure. Unfortunately the majority of these measurements have been made when the electrons are in thermal equilibrium with the surrounding gas. Figure 3.15 shows the mobility of electrons in hydrogen vs. density. These data were taken at $77.6 \mathrm{~K}$.

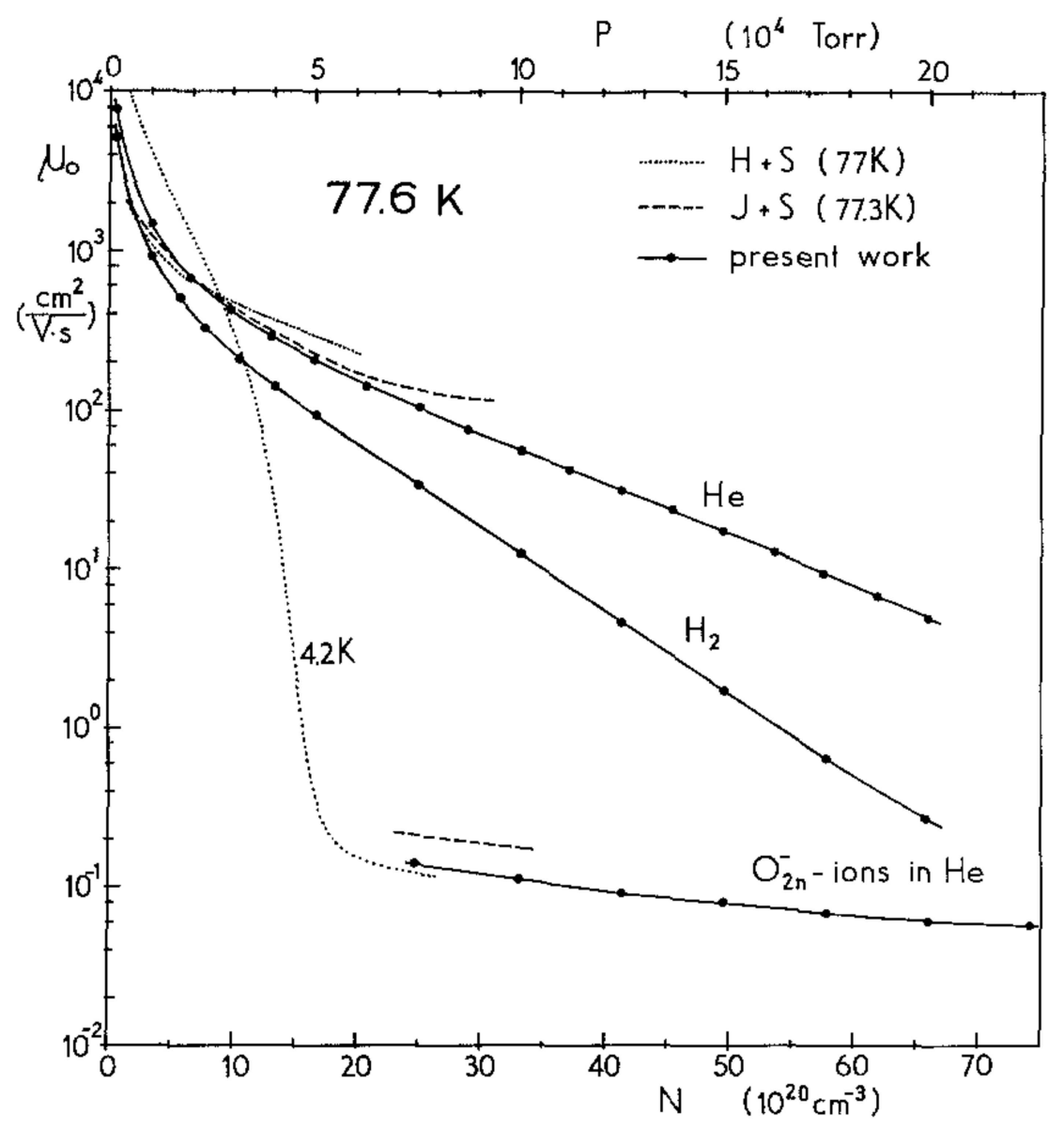

Figure 3.15. Electron mobility in hydrogen vs. density, taken at $77.6 \mathrm{~K}$ [54].

At $77.6 \mathrm{~K}$, our range of densities would be $\approx 1.3-6.7 \times 10^{20} \mathrm{~cm}^{-3}$. Over this range in Fig. 3.15 the mobility drops by almost a factor of 10 . The data taken at room temperature indicate variations in the drift velocity up to $30 \%$ [121]. Figure 3.16 shows the reciprocal of the normalized drift velocity (to the lowest pressure) vs. gas pressure (up to $\approx 600 \mathrm{psi}$ ). It is apparent that the mobility of electrons in hydrogen gets smaller for larger pressure and smaller $X$, as indicated by our results as well. 


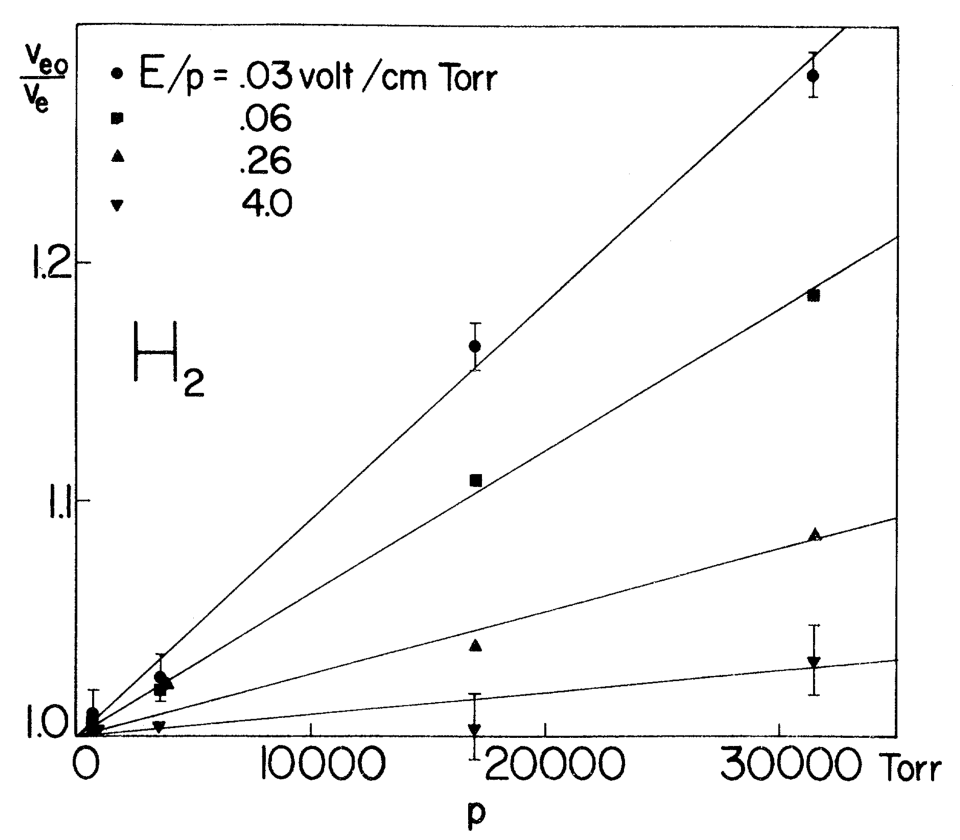

Figure 3.16. The reciprocal of the normalized drift velocity of electrons in hydrogen vs. pressure [55]. Data was taken at room temperature [121].

There have been several theories put forth to explain this effect [55]. The possibility of dimer formation $\left(\mathrm{H}_{2}+2 \mathrm{H}_{2} \rightarrow\left(\mathrm{H}_{2}\right)_{2}+\mathrm{H}_{2}\right)$ changing the mean free path of an electron has been rejected because the electron energy is too high to form a bound state. However, if an electron is captured by a hydrogen molecule while drifting (going into a rotationally excited state of $\mathrm{H}_{2}^{-}$), then released, the effective mobility would decrease because $\mathrm{H}_{2}^{-}$has a much lower mobility than $e^{-}$. The frequency with which an electron is captured and released would increase with increasing pressure. This seems a reasonable explanation. It is worth noting that the energy loss seems to saturate at high pressure. For a 6D Muon Collider cooling-channel using HPRF cavities with pressures up to 2650 psi (180 atm), this is a beneficial effect.

A fit of $d w$ vs. $X$ was done for each pressure. Table 3.1 shows the results.

With the addition of a dopant gas, this method of calculating the energy loss of electrons and ions no longer holds because the electrons get captured by electronegative molecules. However there are no additional sources of charge in the cavity, and 
Table 3.1. Fits of $d w$ for pure hydrogen of the form $d w=c_{1} X^{c_{2}}$.

\begin{tabular}{lll}
\hline \hline Pressure $(\mathrm{psi})$ & $c_{1}$ & $c_{2}$ \\
\hline 300 & $8.106 \times 10^{-15}$ & 1.685 \\
500 & $1.060 \times 10^{-14}$ & 1.611 \\
800 & $1.649 \times 10^{-14}$ & 1.603 \\
950 & $2.089 \times 10^{-14}$ & 1.643 \\
1100 & $2.268 \times 10^{-14}$ & 1.635 \\
1300 & $3.155 \times 10^{-14}$ & 1.678 \\
1470 & $3.628 \times 10^{-14}$ & 1.687 \\
1520 & & \\
\hline
\end{tabular}

the number of positively charged particles is always equal to the number of negatively charged particles, and so an energy loss estimate can be made when hydrogen is doped with dry air. This will now contain the energy loss due to any remaining electrons, plus that of $\mathrm{H}_{n}^{+}$and $\mathrm{O}_{2}^{-}$. For the most part only the ions will contribute, as oxygen will have captured the majority (if not all) of the electrons. As long as ion-ion recombination has not had enough time to neutralize the plasma, measurements of the ion energy loss can be made.

When dry air is added to the cavity, two additional corrections to the number of electron-ion pairs produced must be made. Both corrections are made based on a weighted sum. First, the effective $d E / d x$ increases with increasing DA concentration (for hydrogen; $\frac{d E}{d x}=6.33 \frac{\mathrm{MeV} \mathrm{cm}^{2}}{\mathrm{~g}}, \rho=8.376 \times 10^{-5} \frac{\mathrm{g}}{\mathrm{cm}^{3}}$; for DA; $\left.\frac{d E}{d x}=2.687 \frac{\mathrm{MeV} \mathrm{cm}^{2}}{\mathrm{~g}}, \rho=1.205 \times 10^{-3} \frac{\mathrm{g}}{\mathrm{cm}^{3}}\right)$. Figure 3.17 shows the correction factor applied based on DA concentration. Second, the effective energy required to ionize 
a molecule decreases slightly (for hydrogen; $W_{i}=36.2 \mathrm{eV}$; for DA; $W_{i}=31.7 \mathrm{eV}$ ).

Figure 3.18 shows this correction vs. DA concentration.

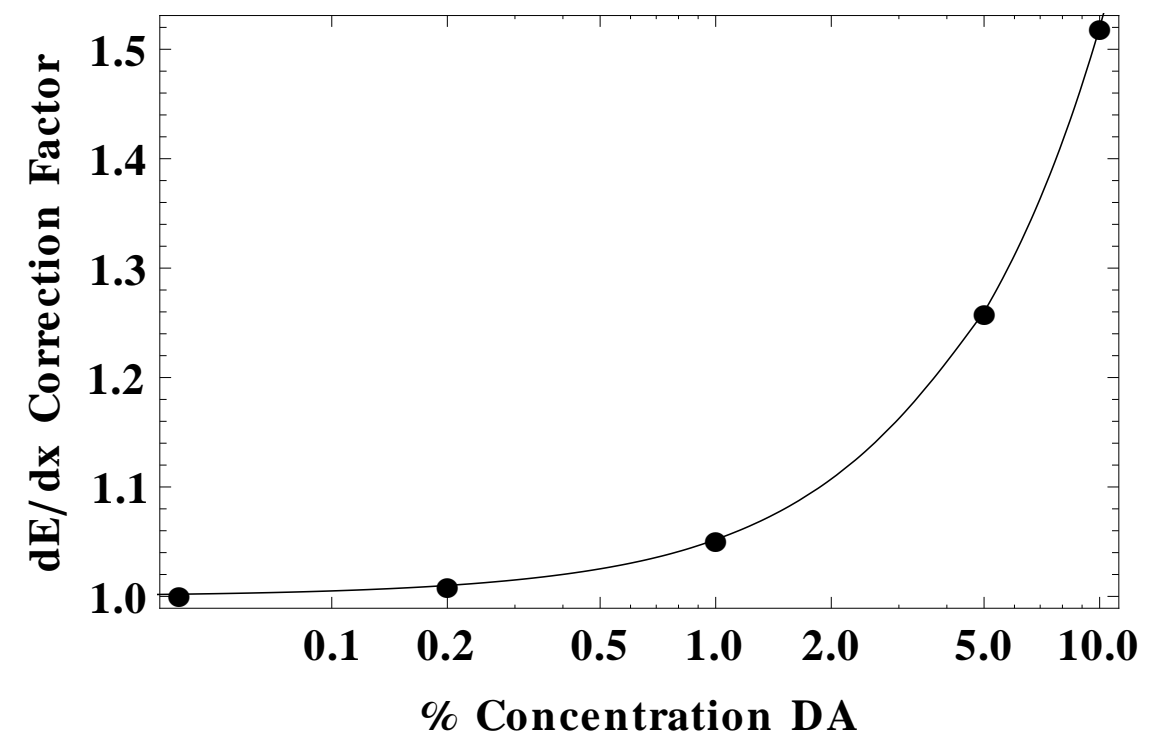

Figure 3.17. Correction to $d E / d x$ with dry air dopant.

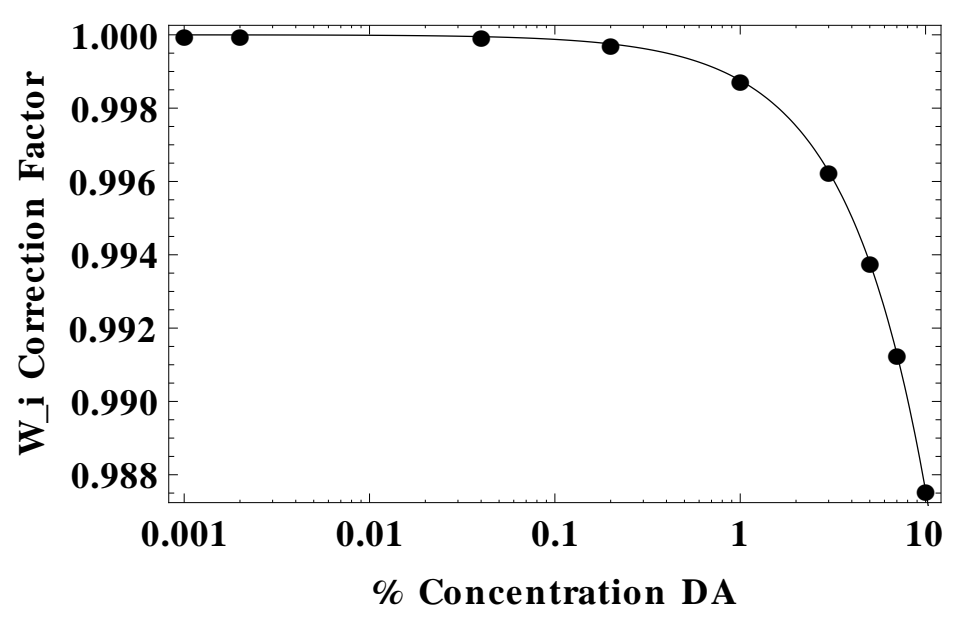

Figure 3.18. Correction to ionization energy with dry air dopant.

Figures 3.19 through 3.25 show the measured energy loss in the cavity. Each plot is for a given pressure and shows various DA concentrations. The lines are the model prediction for the energy loss based on two extremes: only electrons contribute (assuming none are not captured); and only ions $\left(\mathrm{H}_{5}^{+}\right.$and $\left.\mathrm{O}_{2}^{-}\right)$contribute (assuming all of the electrons are captured. 


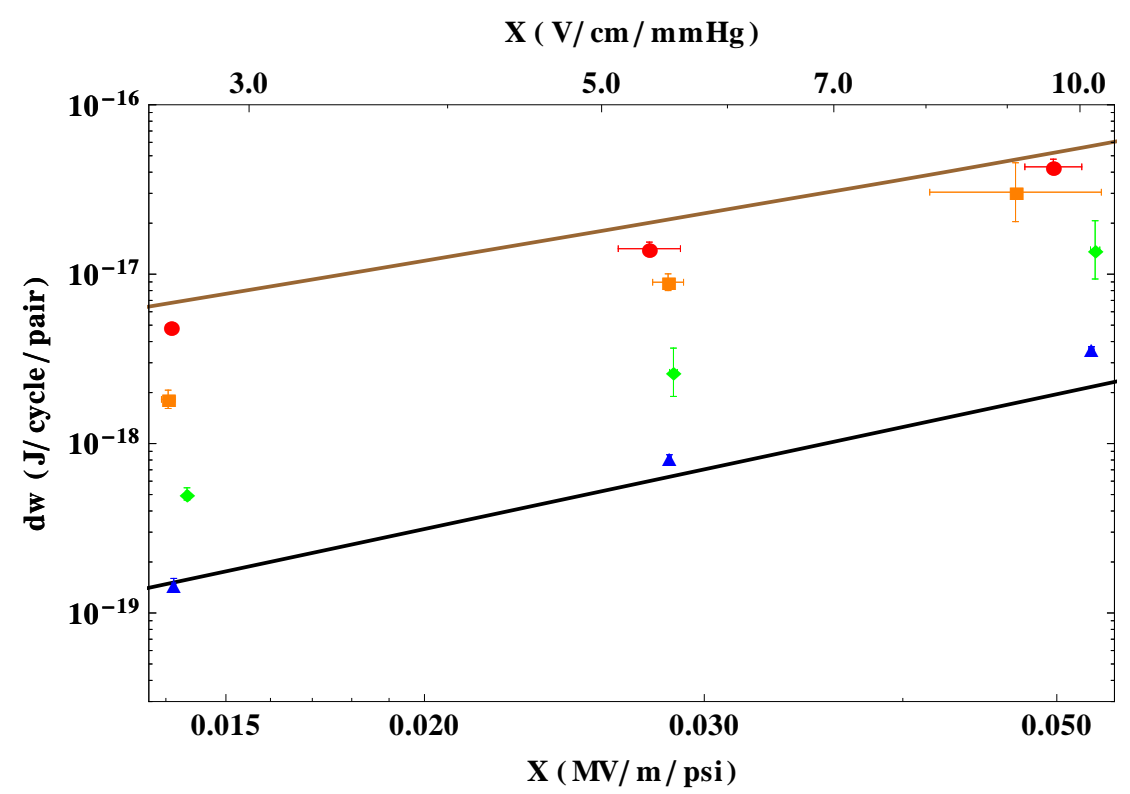

Figure 3.19. $d w$ for $300 \mathrm{psi}$ hydrogen doped with dry air. The colors correspond to varying concentrations: $0.04 \%$ (red), $0.2 \%$ (orange), $1 \%$ (green), and $5 \%$ (blue). The brown line is the model prediction for electrons and the black line is the model prediction for $\mathrm{H}_{5}^{+}$and $\mathrm{O}_{2}^{-}$.

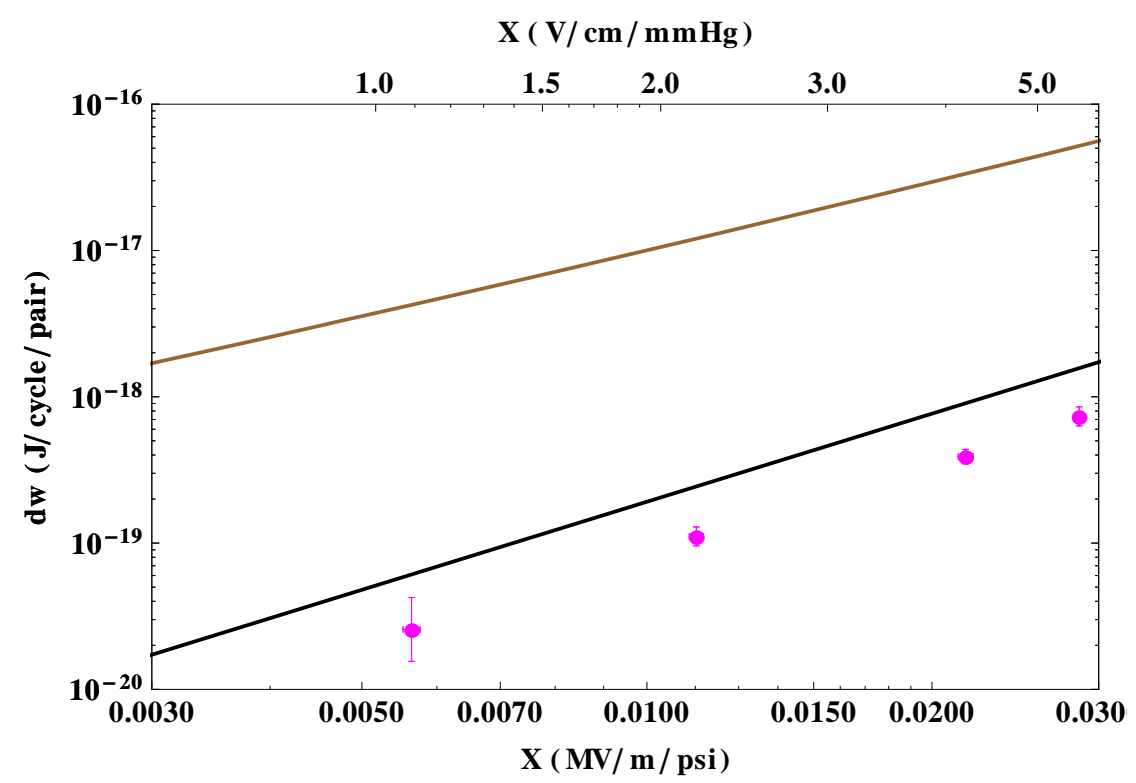

Figure 3.20. $d w$ for 735 psi hydrogen doped with dry air. Data points (magenta) correspond to $10 \% \mathrm{DA}$ concentration. The brown line is the model prediction for electrons and the black line is the model prediction for $\mathrm{H}_{5}^{+}$and $\mathrm{O}_{2}^{-}$. 


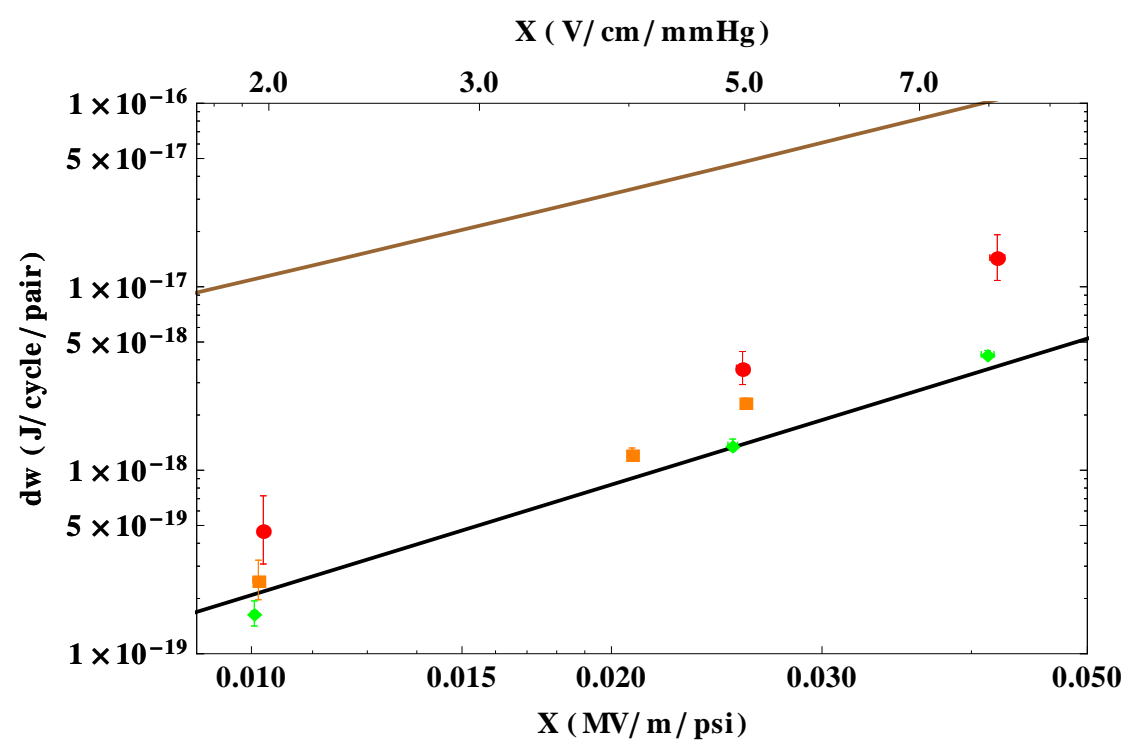

Figure 3.21. $d w$ for 800 psi hydrogen doped with dry air. The colors correspond to varying concentrations: $0.04 \%$ (red), $0.2 \%$ (orange), and $1 \%$ (green). The brown line is the model prediction for electrons and the black line is the model prediction for $\mathrm{H}_{5}^{+}$and $\mathrm{O}_{2}^{-}$.

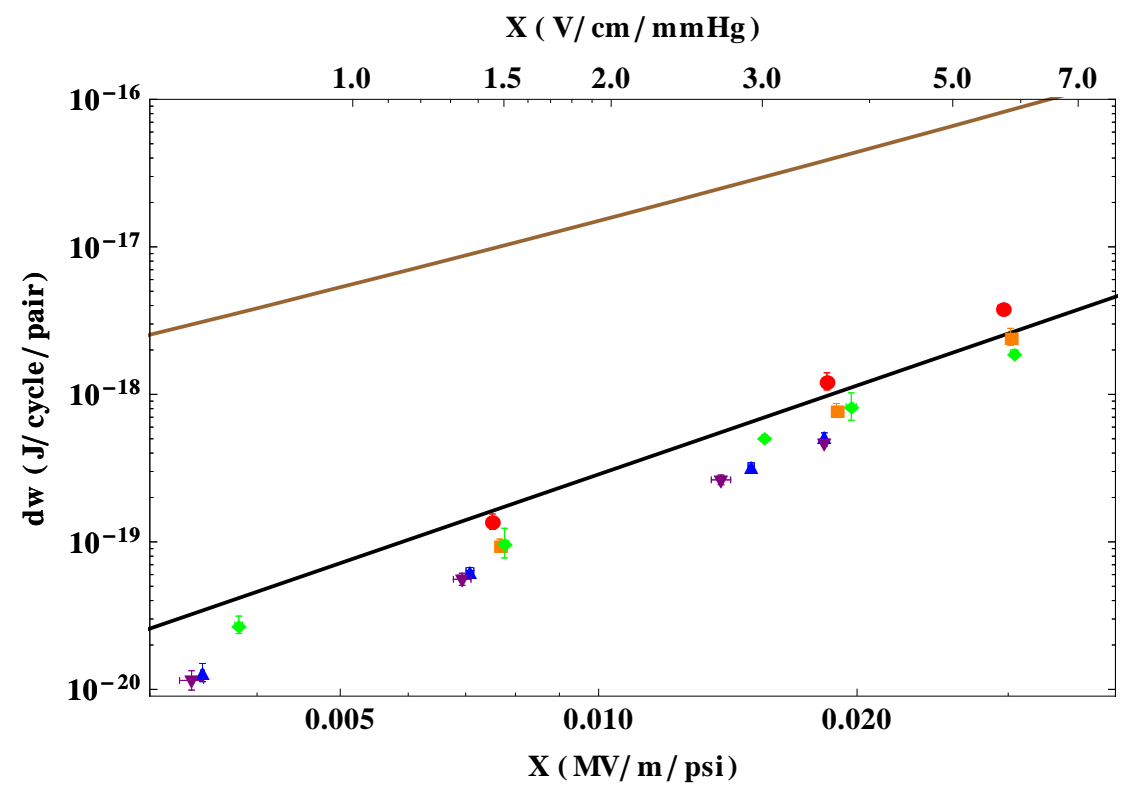

Figure 3.22. $d w$ for 1100 psi hydrogen doped with dry air. The colors correspond to varying concentrations: $0.04 \%$ (red), $0.2 \%$ (orange), $1 \%$ (green), $5 \%$ (blue), and $6.78 \%$ (purple). The brown line is the model prediction for electrons and the black line is the model prediction for $\mathrm{H}_{5}^{+}$and $\mathrm{O}_{2}^{-}$. 


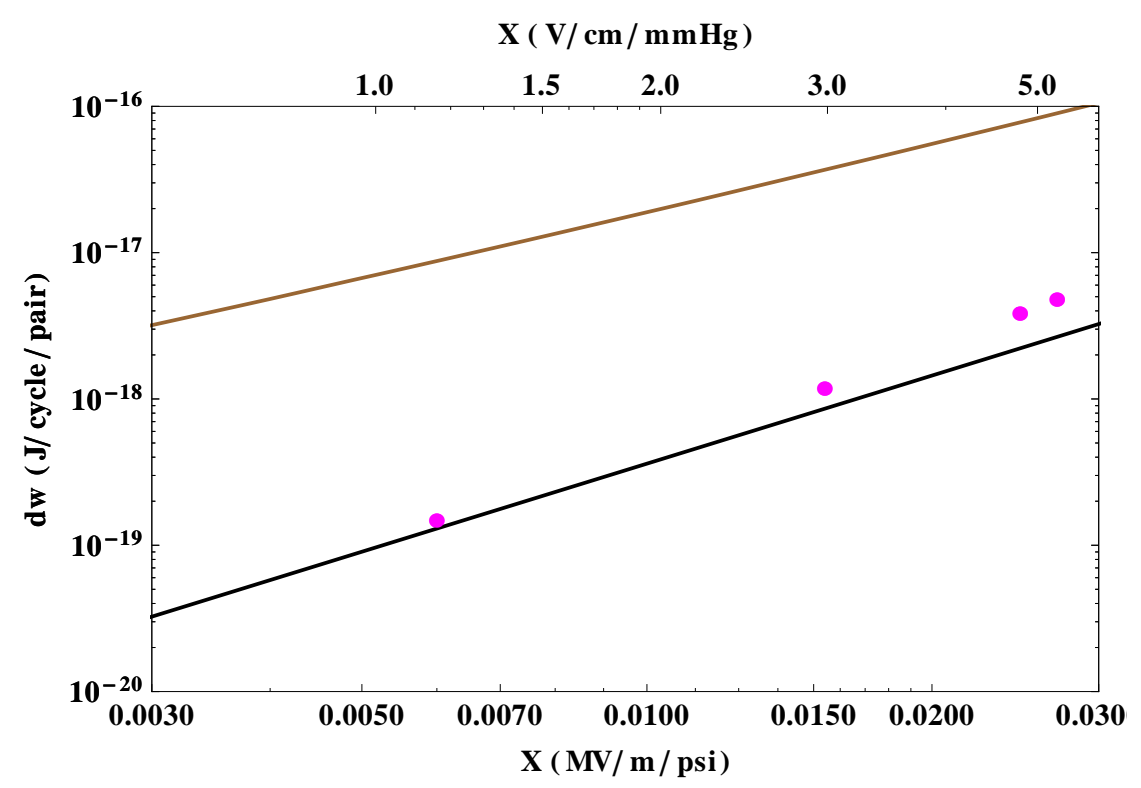

Figure 3.23. $d w$ for 1385 psi hydrogen doped with dry air. The data points (magenta) correspond to $0.022 \%$ DA concentration. The brown line is the model prediction for electrons and the black line is the model prediction for $\mathrm{H}_{5}^{+}$and $\mathrm{O}_{2}^{-}$.

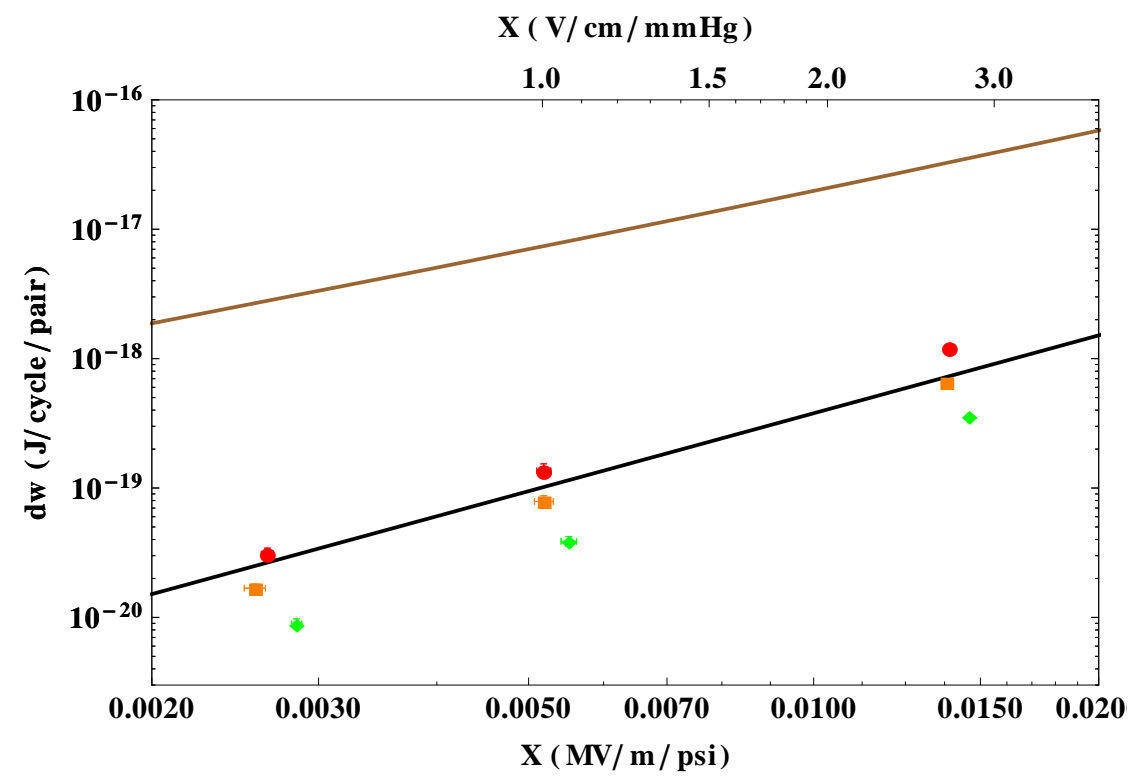

Figure 3.24. $d w$ for 1450 psi hydrogen doped with dry air. The colors correspond to varying concentrations: $0.04 \%$ (red), $0.2 \%$ (orange), $5 \%$ (green). The brown line is the model prediction for electrons and the black line is the model prediction for $\mathrm{H}_{5}^{+}$and $\mathrm{O}_{2}^{-}$. 


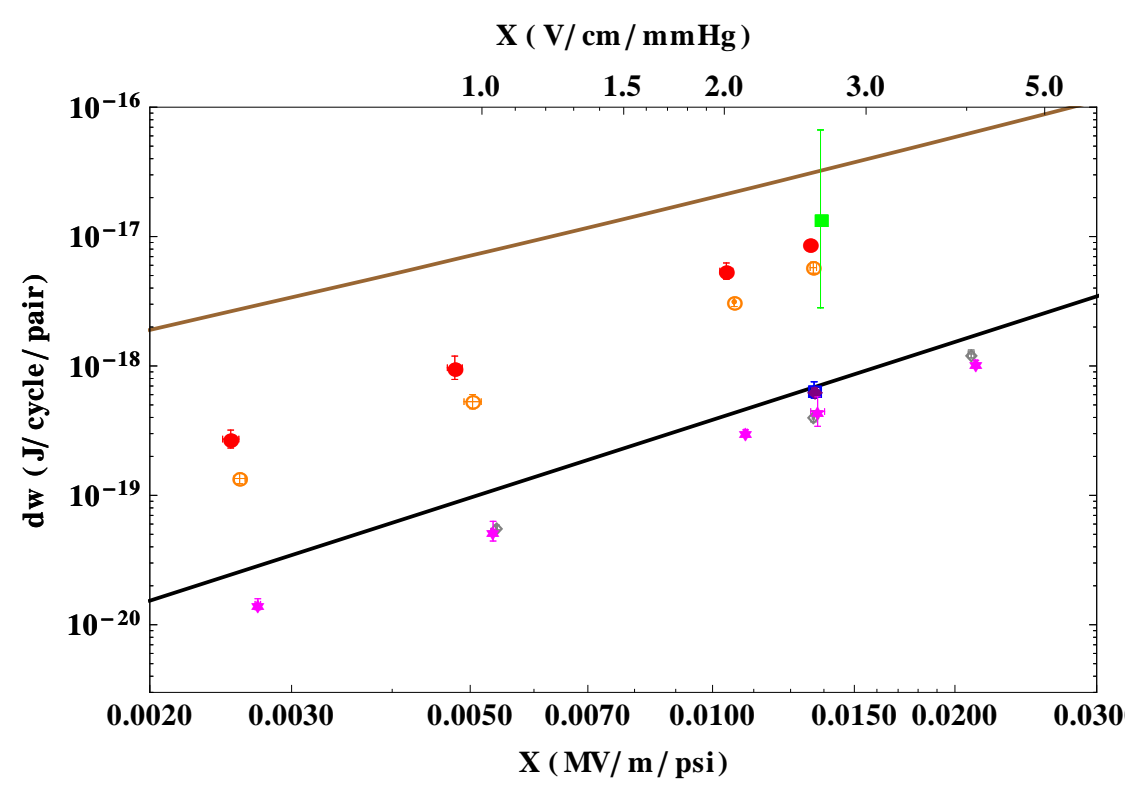

Figure 3.25. $d w$ for 1470 psi hydrogen doped with dry air. The colors correspond to varying concentrations: $0.001 \%$ (red), $0.002 \%$ (orange), $0.01 \%$ (green), $0.025 \%$ (blue), $0.05 \%$ (purple), $0.2 \%$ (gray), and $1 \%$ (magenta). The brown line is the model prediction for electrons and the black line is the model prediction for $\mathrm{H}_{5}^{+}$ and $\mathrm{O}_{2}^{-}$.

It can be seen that at 300 psi, the energy loss falls somewhere between those for pure electrons and pure ions, with increasing DA concentration decreasing the energy loss. This would indicate that there are residual electrons in the cavity. For the case of 1470 psi, only very small DA concentrations $(0.001 \%$ and $0.002 \%)$ leave residual electrons in the cavity. Once the concentration reaches $0.025 \%$, the energy loss appears to be coming entirely from ions. Note that in many of the plots the energy loss measured is less than that predicted. A constant mobility was used for the $\mathrm{H}_{5}^{+}$and $\mathrm{O}_{2}^{-}$predictions. As we have seen, the mobility, and therefore energy loss, decreases with increasing pressure.

Fits of $d w$ for DA doped hydrogen are given in Tab. 3.2. 
Table 3.2. Fits of $d w$ for DA doped hydrogen of the form $d w=c_{1} X^{c_{2}}$.

\begin{tabular}{|c|c|c|c|}
\hline Pressure (psi) & Concentration (\%) & $c_{1}$ & $c_{2}$ \\
\hline 300 & 0.04 & $6.884 \times 10^{-15}$ & 1.705 \\
\hline 300 & 0.2 & $3.207 \times 10^{-14}$ & 2.286 \\
\hline 300 & 1 & $2.176 \times 10^{-14}$ & 2.516 \\
\hline 300 & 5 & $4.294 \times 10^{-15}$ & 2.407 \\
\hline 735 & 10 & $1.027 \times 10^{-15}$ & 2.044 \\
\hline 800 & 0.04 & $2.584 \times 10^{-14}$ & 2.390 \\
\hline 800 & 0.2 & $1.113 \times 10^{-14}$ & 2.333 \\
\hline 800 & 1 & $6.568 \times 10^{-15}$ & 2.302 \\
\hline 1100 & 0.04 & $2.043 \times 10^{-14}$ & 2.436 \\
\hline 1100 & 0.2 & $9.629 \times 10^{-15}$ & 2.369 \\
\hline 1100 & 1 & $2.820 \times 10^{-15}$ & 2.084 \\
\hline 1100 & 5 & $3.503 \times 10^{-15}$ & 2.206 \\
\hline 1100 & 6.78 & $3.009 \times 10^{-15}$ & 2.190 \\
\hline 1385 & 0.022 & $1.990 \times 10^{-14}$ & 2.310 \\
\hline 1450 & 0.04 & $1.502 \times 10^{-14}$ & 2.207 \\
\hline 1450 & 0.2 & $7.334 \times 10^{-15}$ & 2.177 \\
\hline 1450 & 5 & $4.930 \times 10^{-15}$ & 2.257 \\
\hline 1470 & 0.001 & $8.014 \times 10^{-14}$ & 2.109 \\
\hline 1470 & 0.002 & $1.033 \times 10^{-13}$ & 2.284 \\
\hline 1470 & 0.2 & $7.493 \times 10^{-15}$ & 2.2612 \\
\hline 1470 & 1 & $4.298 \times 10^{-15}$ & 2.140 \\
\hline
\end{tabular}


3.1.1 Energy Loss Uncertainties. The vertical error bars in Fig. 3.12 - 3.14 are statistical and are $<5 \%$. There are three main sources of systematic errors. The first comes from the measurement of the gas pressure, which was performed using an analog gauge. Each time the cavity was pressurized, sufficient time elapsed so as to allow the system pressure to reach an equilibrium. Assuming the gauge was calibrated to a few psi, and the operator pressurized the cavity to within a few psi of the desired pressure, this represents at most a few percent error. This would be reflected (scale linearly) in the calculation of both $X$ and the number of electron-ion pairs produced.

The second comes from the power measurement. This relies on the pickup signal from the cavity. The magnetic pickup probe was used for calculations. Figure 3.26 shows the difference in results obtained from the electric and magnetic pickups. The electric probe data are within $10 \%$ of the magnetic probe data. Finally, during the initial drop in voltage in the cavity, the second term of Eq. 3.1 $\left(C V \frac{d V}{d t}\right)$ is comparable to the first, and so the capacitance of the cavity $\left(C=1 /\left(L \omega^{2}\right)\right)$ plays a significant role. The cavity was tuned to within a few $\mathrm{kHz}$ of the resonant frequency at all times. In addition, the frequency was stable for each setting so the capacitance remained the same. The inductance was calculated using Superfish, and is accurate to a few percent. The error in the power measurement is dominated by the accuracy of the pickup probes, $<10 \%$.

The third source of systematic error comes from the beam intensity measurement. As mentioned previously, it was not possible to make an in-situ calibration due to the experimental setup. The beam current is calculated from a resistive and inductive term. Any additional resistance or inductance in the system is introduced by the signal cables. The cable attenuation contributes, but has been corrected for [113]. The toroid calibration introduces $3 \%$ uncertainty into the beam current measurement. 


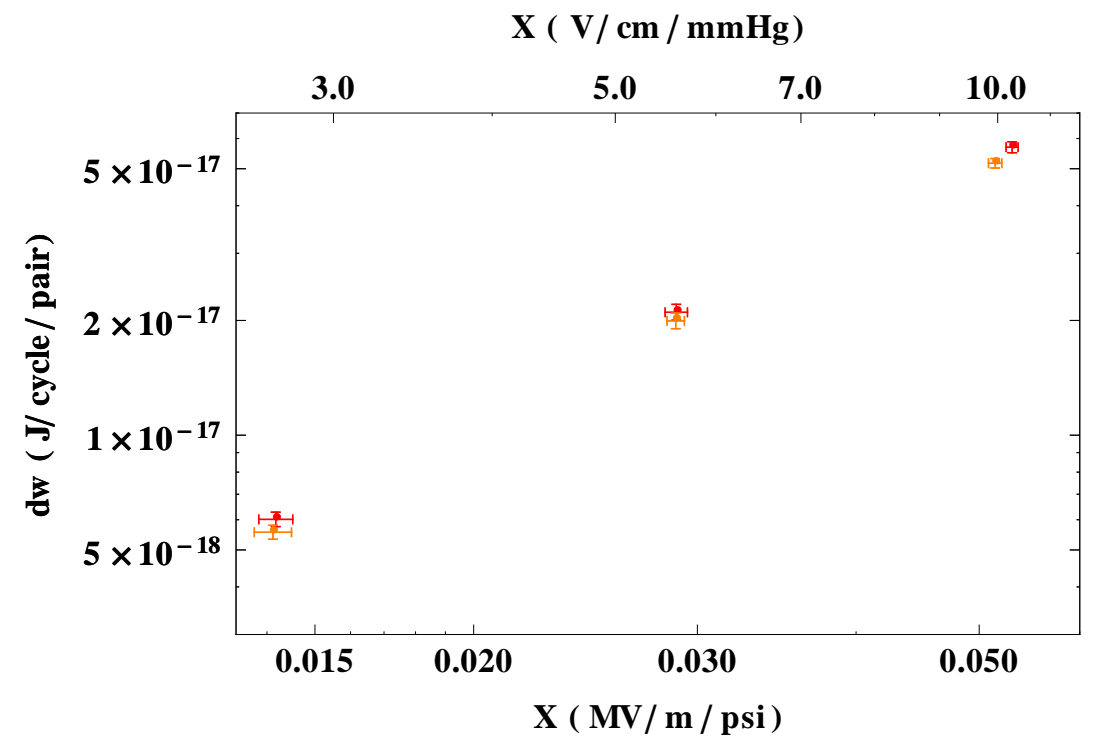

Figure 3.26. Hydrogen 300 psi data $d w$ comparison between the electric (orange) and magnetic (red) pickup probes.

The cable attenuation and inductance accounts for $5 \%$ uncertainty. Noise in the signal at the beginning of the beam (which effects the calcuted time at which the beam turns on) can contribute $2 \%$. The total uncertainty is then on the order of $10 \%$.

There is also the digitizing error in the scope, which is on the order of $0.15 \%$, and is present in every measurement. Table 3.3 summarizes the various sources of error. Figure 3.27 shows the data with the statistical error (as in Fig. 3.14) with $\pm 15 \%$ systematic error added linearly. 


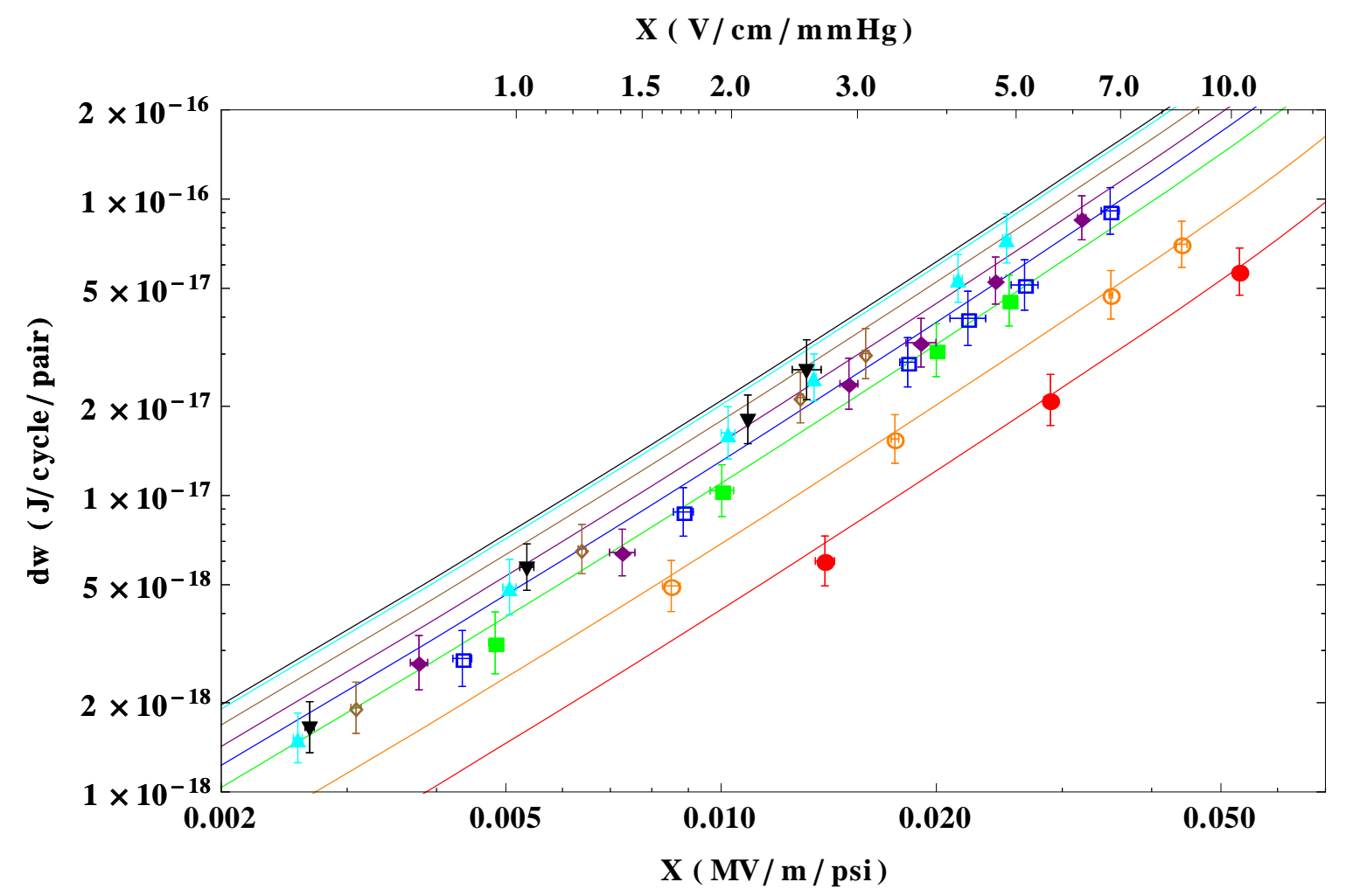

Figure 3.27. Energy loss for pure hydrogen including systematic errors. Colors correspond to pressures: 300 psi (red), 500 psi (orange), 800 psi (green), 950 psi (blue), 1100 psi (purple), 1300 psi (brown), 1470 psi (cyan), and 1520 psi (black). 
Table 3.3. Errors on $d w$.

\begin{tabular}{lll}
\hline \hline Source & Affects & Estimate $(\%)$ \\
\hline Gas pressure & Calculation of $X$ & $0.1-1$ \\
& $N_{\text {pairs }}$ & $0.1-1$ \\
Pickup probe calibration & Power & 10 \\
Cavity capacitance & Power & 3 \\
Toroid calibration & Beam current & 3 \\
Cable attenuation & Beam current & 5 \\
\hline
\end{tabular}




\subsection{Recombination}

In pure hydrogen, the recombination rate has been measured. As we have no way of distinguishing $\mathrm{H}_{3}^{+}$from $\mathrm{H}_{5}^{+}$, what we measure is the effective recombination rate, $\beta$, and from Eq. 1.33:

$$
\begin{aligned}
n_{e} & =n_{\mathrm{H}_{3}^{+}}+n_{\mathrm{H}_{5}^{+}}=n_{H}=n \\
\frac{d n}{d t} & =\dot{n}-\beta_{3} n_{e} n_{\mathrm{H}_{3}^{+}}-\beta_{5} n_{e} n_{\mathrm{H}_{5}^{+}} \\
\frac{d n}{d t} & =\dot{n}-\beta n^{2} \\
\beta & =\frac{\beta_{3} n_{\mathrm{H}_{3}^{+}}+\beta_{5} n_{\mathrm{H}_{5}^{+}}}{n_{\mathrm{H}_{3}^{+}}+n_{\mathrm{H}_{5}^{+}}}
\end{aligned}
$$

where $\beta_{m}$ is the recombination rate for $\mathrm{H}_{m}^{+}$. If $n_{\mathrm{H}_{5}^{+}} \gg n_{\mathrm{H}_{3}^{+}}$then the effective recombination rate is essentially that of $\mathrm{H}_{5}^{+}$. When the system is in equilibrium (the power being fed into the gas is equal to the power being absorbed), the voltage is at a minimum, $d n_{e} / d t=0$ and

$$
\begin{aligned}
\dot{n} & =\beta n^{2} \\
\beta & =\frac{\dot{n}}{n^{2}}
\end{aligned}
$$

To calculate $\beta$, the production rate and number density of electron-ion pairs must be measured. The number of electron-ion pairs produced can be found as shown in Sec. 3.1.

At this point it is important to note that we have no way of directly measuring the number density of electrons or ions in the cavity. What we can do is measure the total number, and then simulate the distribution of electrons and ions, using G4beamline, to get an effective volume that the plasma occupies. Figure 3.28 shows a simulation of the beam passing through the cavity.

The number density can be given in terms of a distribution function, $\rho$ :

$$
n(r, z)=n_{0} \rho(r, z)
$$




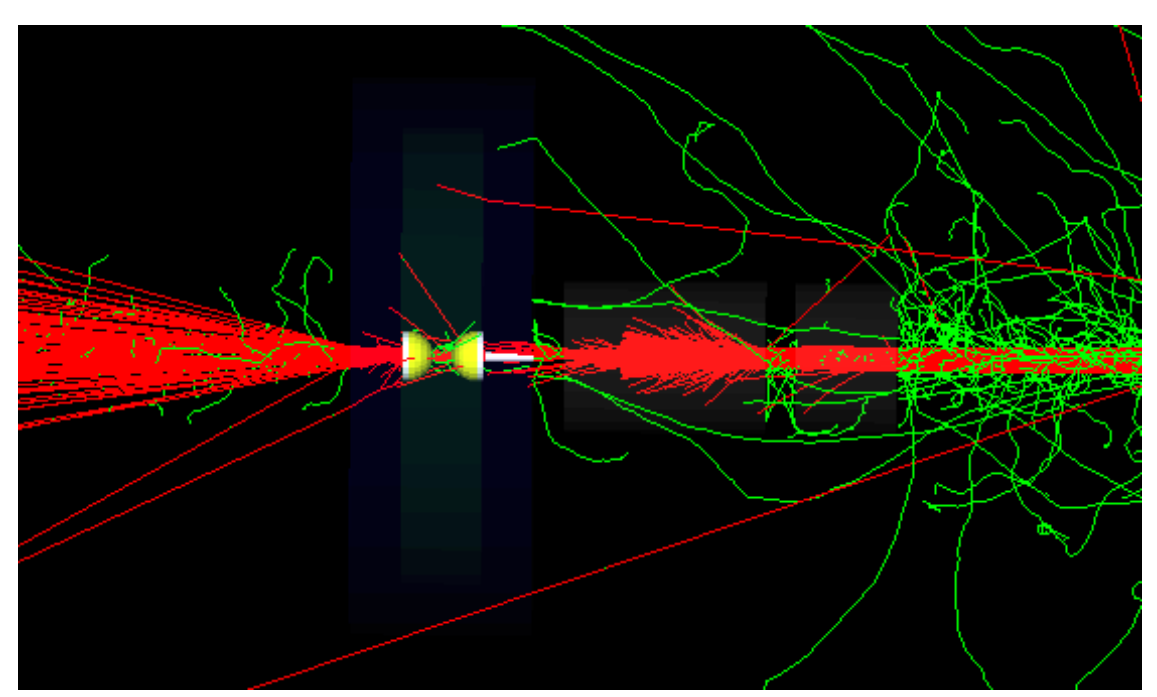

Figure 3.28. A simulation of the beam (red, and electrons in green) traversing the HPRF cavity (blue). The beam enters from the right, traveling through air. The two collimators are shown in gray. The electrodes (yellow) and counterbore in the upstream face of the cavity (white) are also shown.

where $n_{0}$ is the total number of particles and $\int \rho(r, z) d V=1$. It is assumed that the density functions of electrons and ions are the same and that $\beta$ is constant throughout the volume (this isn't entirely true, but is acceptable as a first approximation). To calculate $\beta$ at equilibrium, one uses Eq. 3.10 along with the plasma distribution:

$$
\begin{aligned}
n & =\sqrt{\frac{\dot{n}}{\beta}} \\
n_{0} \rho(r, z) & =\sqrt{\frac{\dot{n}_{0} \rho(r, z)}{\beta}} \\
\int n_{0} \rho(r, z) d V & =\sqrt{\frac{\dot{n}_{0} \rho(r, z)}{\beta}} d V \\
n_{0} & =\sqrt{\frac{\dot{n}_{0}}{\beta} \int \sqrt{\rho(r, z)}} d V \\
\beta & =\frac{\dot{n}_{0}}{n_{0}^{2}}\left(\int \sqrt{\rho(r, z)} d V\right)^{2}
\end{aligned}
$$

Based on the simulation, $\left(\int \sqrt{\rho(r, z)} d V\right)^{2}=0.526 \mathrm{~cm}^{3}$. Using the length of the gap between electrodes $(1.77 \mathrm{~cm})$, this corresponds to an effective plasma radius of $3.08 \mathrm{~mm}$, approximately one and a half times the radius of the through-hole in the 
downstream collimator.

The number of electron-ion pairs present at equilibrium can be calculated from the power fed to the gas (averaged over $200 \mathrm{~ns}$ ) and the measured energy loss at the equilibrium voltage:

$$
n_{0}=\frac{P_{g}}{d w f}
$$

Figure 3.29 shows the recombination rates for all pressures of pure hydrogen vs. the equilibrium $X$. There appears to be a difference between high ( $>10^{10}$ protons $/$ pulse) and low beam intensity data.

There appears to be a small dependence on pressure. It can be seen that $\beta$ decreases with increasing $X$. The recombination rates are consistent with those presented in Sec. 1.2.5 for $\mathrm{H}_{5}^{+}$. It should be pointed out that, especially for the high beam intensity and low pressure data, the electric fields at which the measurements were made could be very small (in some cases $<1 \mathrm{MV} / \mathrm{m}$ ), therefore the signal to noise ratio is much higher.

Table 3.4 shows the fits to the equilibrium recombination rate data. 


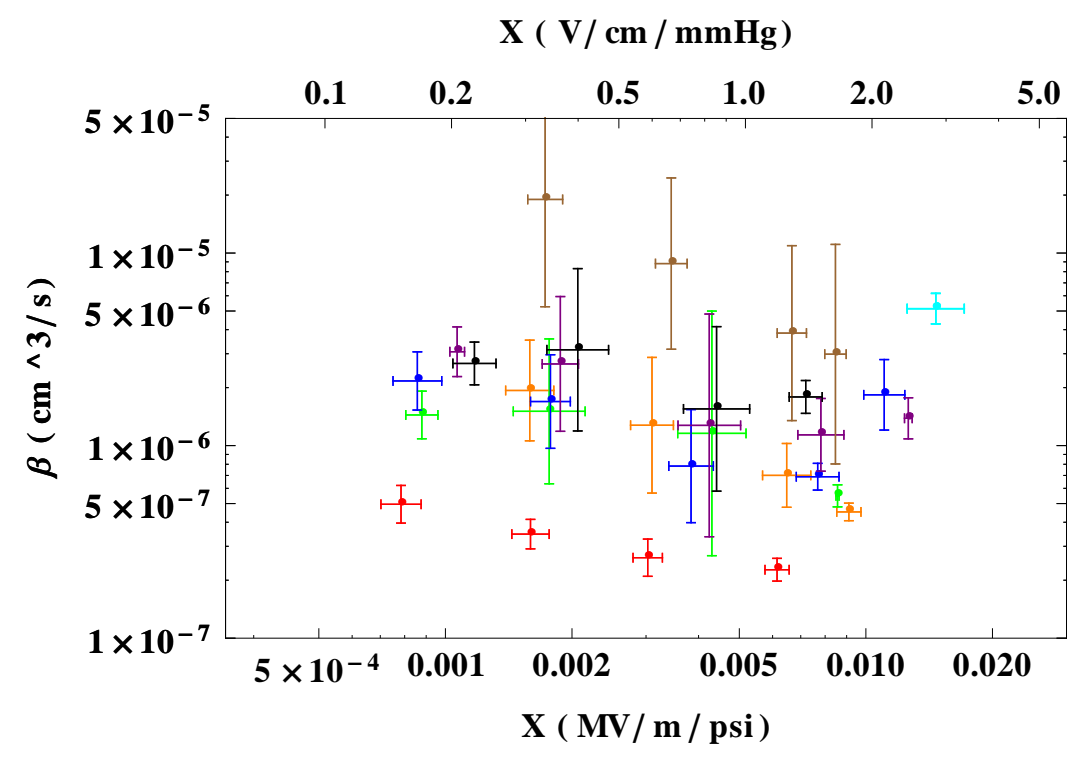

(a). Low beam intensity.

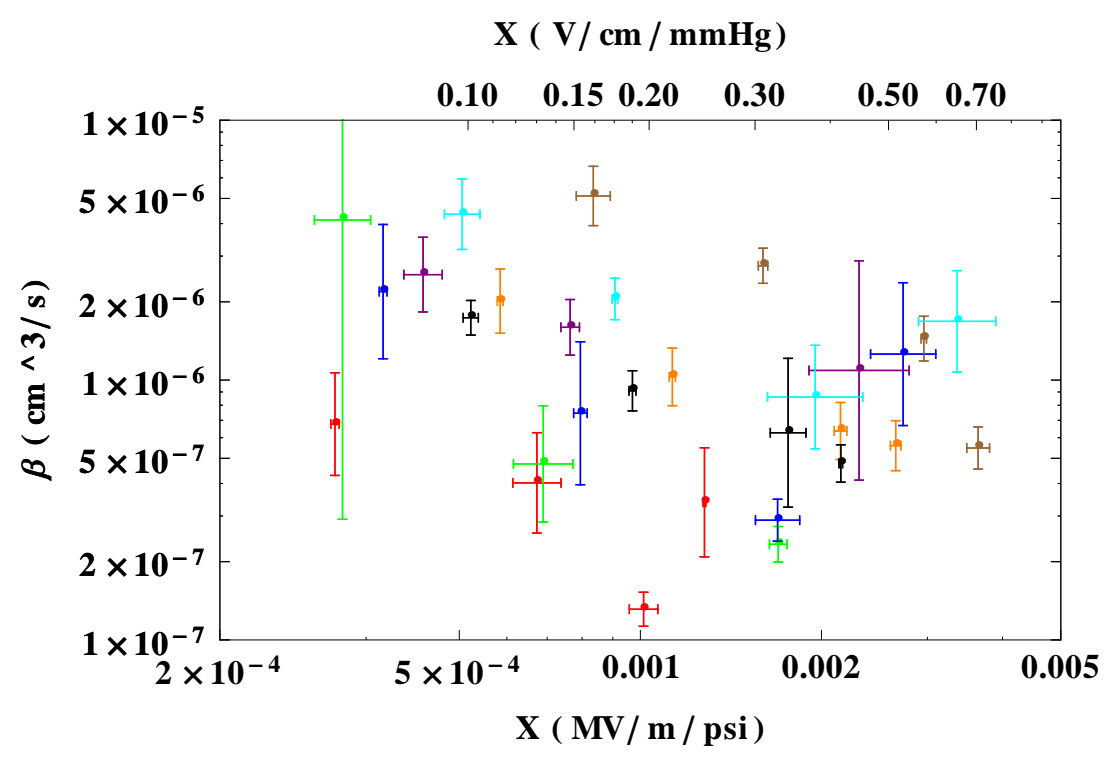

(b). High beam intensity.

Figure 3.29. Recombination rates measured at equilibrium for pure hydrogen at different pressures: 300 psi (red), 500 psi (orange), 800 psi (green), 950 psi (blue), 1100 psi(purple), 1300 psi (brown), 1470 psi (cyan), and 1520 psi (black). 
Table 3.4. Fits of $\beta$ for pure hydrogen of the form $\beta=c_{1} X^{-c_{2}}$.

\begin{tabular}{|c|c|c|c|}
\hline Pressure (psi) & Beam intensity & $c_{1}$ & $c_{2}$ \\
\hline 300 & High & $4.083 \times 10^{-8}$ & 1.193 \\
\hline 300 & Low & $2.710 \times 10^{-7}$ & 0.389 \\
\hline 500 & High & $6.752 \times 10^{-7}$ & 0.866 \\
\hline 500 & Low & $1.6534 \times 10^{-6}$ & 0.827 \\
\hline 800 & High & $2.670 \times 10^{-7}$ & 1.649 \\
\hline 800 & Low & $1.307 \times 10^{-6}$ & 0.426 \\
\hline 950 & High & $5.890 \times 10^{-7}$ & 1.225 \\
\hline 950 & Low & $1.919 \times 10^{-6}$ & 0.437 \\
\hline 1100 & High & $1.544 \times 10^{-6}$ & 0.612 \\
\hline 1100 & Low & $3.219 \times 10^{-6}$ & 0.391 \\
\hline 1300 & High & $6.066 \times 10^{-6}$ & 1.289 \\
\hline 1300 & Low & $4.916 \times 10^{-6}$ & 1.167 \\
\hline 1470 & High & $2.990 \times 10^{-6}$ & 0.949 \\
\hline 1520 & High & $1.382 \times 10^{-6}$ & 0.919 \\
\hline 1520 & Low & $3.157 \times 10^{-6}$ & 0.247 \\
\hline
\end{tabular}


Measurements of the recombination rate were also made using a different method. A recursion formula was applied to Eq.1.33, with half-period time steps for the duration of the beam. The total number of electrons and ions $\left(N_{e}\right.$ and $\left.N_{H}\right)$ at each step were calculated using:

$$
\begin{aligned}
& N_{e}(s+1)=N_{e}(s)+\dot{N}(t(s)) \delta t-\beta(V(t(s))) N_{e}(s) N_{H}(s) \delta t \\
& N_{H}(s+1)=N_{H}(s)+\dot{N}(t(s)) \delta t-\beta(V(t(s))) N_{e}(s) N_{H}(s) \delta t \\
& t(s+1)=t(s)+\frac{1}{2 f} \\
& t(1)=t_{0}, \quad N_{e}(1)=0, \quad N_{H}(1)=0
\end{aligned}
$$

where $\delta t$ is half an RF period, $\dot{N}$ is the derivative of the number of protons given in Fig. 3.4, and $V$ is the voltage in the cavity. Using the number of electrons and ions vs. time and the measured energy loss (Tab.3.1) vs. voltage (and therefore time), the gas power was estimated $\left(P_{g}=d w n f\right)$. Fits to the $\beta$ vs. $X$ curves at a given pressure were used as a starting point, and modified until the resulting power from the recursion formula matched that measured. Each set of electric field, gas pressure, and beam intensity contained data from $\approx 8$ beam pulses. Cuts were made based on electric field and beam intensity (falling within $10 \%$ of the average), and the remaining data points were averaged and then fed into the recursion formula. Figure 3.30 shows the results of the recursion formula.

Notice that for high pressure, recombination begins much sooner than for low pressure, and more electrons recombine. The power dumped into the gas is greater at higher pressure as well. As with the equilibrium method calculation, this produces rates in units of $\mathrm{s}^{-1}$. The same plasma volume that was used to calculate the equilibrium recombination rates was used here as well. The plasma distribution varies in time, due to diffusion, but we will assume it is constant (as will be seen in Sec.3.2.1 the diffusion is very small). As this produces $\beta$ vs. $V$, we will select $\beta$ 's 


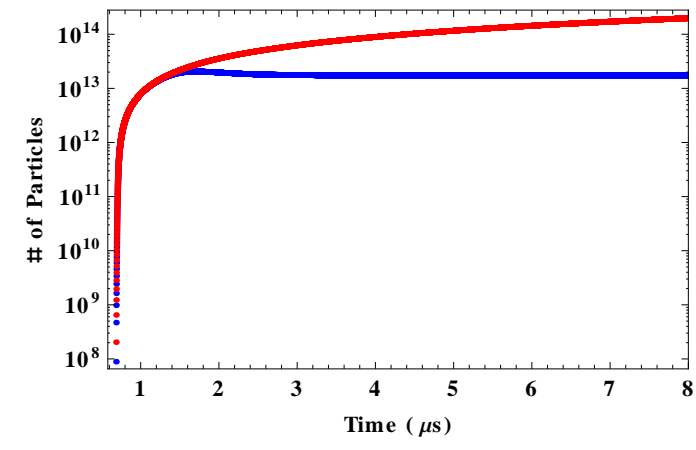

(a). 300 psi hydrogen. The number of particles produced is in red, the number of electrons and ions is in blue.

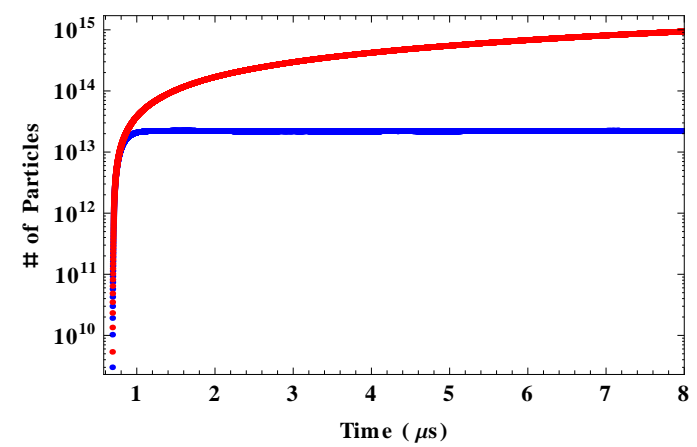

(c). 1470 psi hydrogen. The number of particles produced is in red, the number of electrons and ions is in blue.

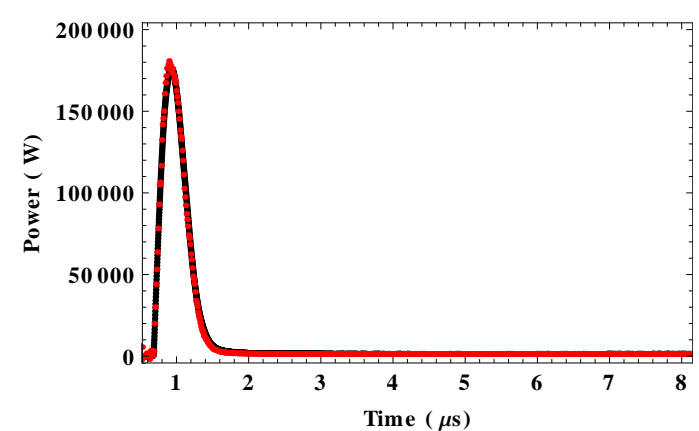

(b). 300 psi hydrogen. The measured power is in red, the calculated power is in black.

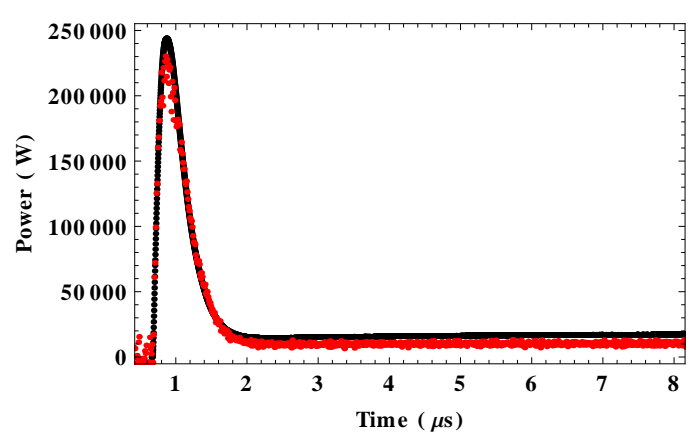

(d). 1470 psi hydrogen. The measured power is in red, the calculated power is in black.

Figure 3.30. Results of the recursion formula for pure hydrogen. The plots on the left are the number of particles in the cavity vs. time, and on the right are the gas power vs. time. 
that correspond the the initial voltage in the cavity.

When the recombination rates based on the equilibrium measurements and recursion formula method are combined, Fig. 3.31 is the result. The equilibrium measurements are those with (statistical) error bars, the recursion calculations are the diamond points, and the dashed line is a prediction based on data from [76]. Our $X$ was used with the data in Fig. 1.23 to determine the kinetic energy of the electrons, which was then converted to a temperature and used with the data from Fig. 1.24 for $\mathrm{H}_{5}^{+}$to produce the dashed line in Fig. 3.31. 


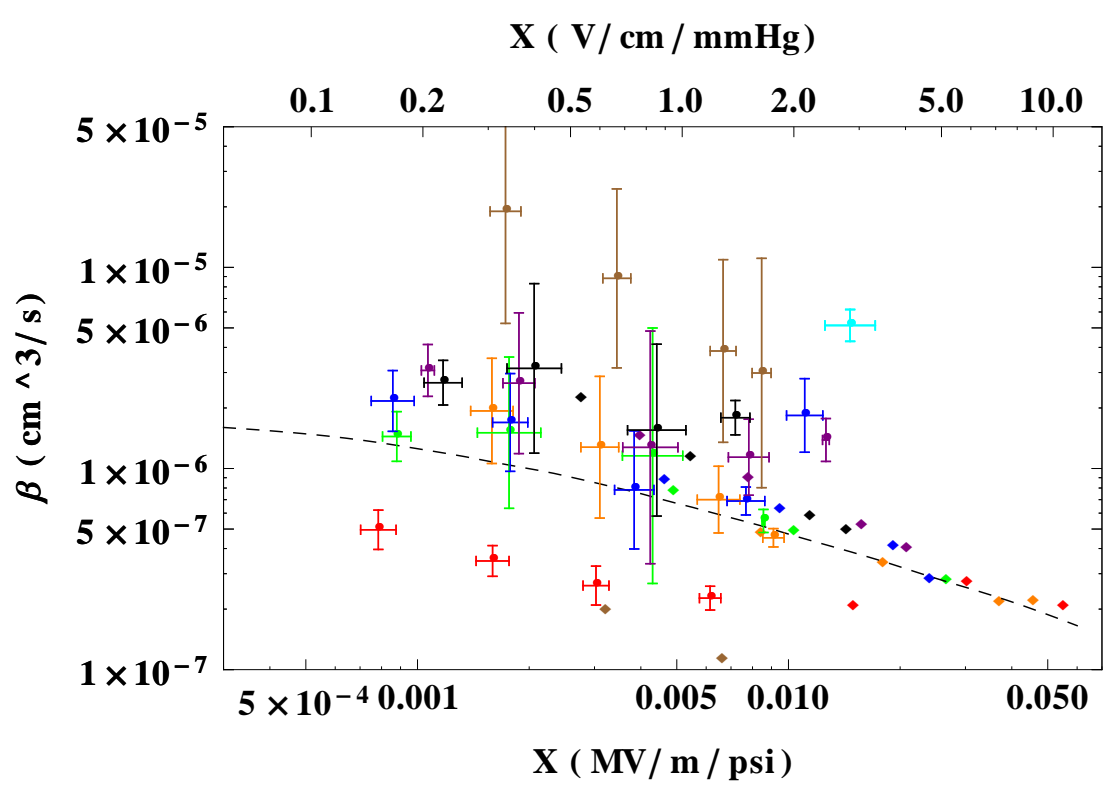

(a). Low beam intensity recombination rates.

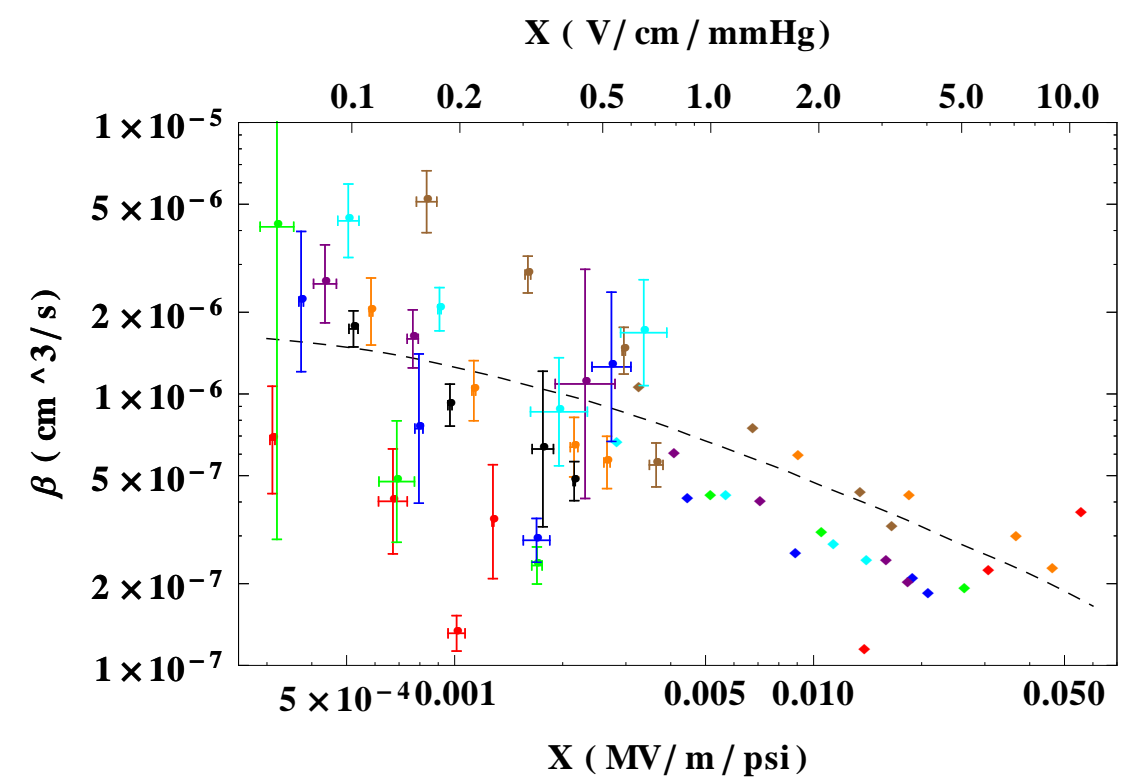

(b). High beam intesensity recombination rates.

Figure 3.31. Recombination rates for pure hydrogen using the equilibrium (points with error bars) and recursion (diamond points) data. All gas pressures are represented: 300 psi (red), 500 psi (orange), 800 psi (green), 950 psi (blue), 1100 psi (purple), 1300 psi (brown), 1470 psi (cyan), and 1520 psi (black). The dashed line is a prediction based on the data from [76]. 
Notice that the data from the recursion formula have less spread with respect to pressure than those from the equilibrium data. This is most likely because (especially for high beam intensities) the electric field at which the measurements were being taken was very low (in some cases $<1 \mathrm{MV} / \mathrm{m}$ ). As all of the data fall very near the recombination rate predicted for $\mathrm{H}_{5}^{+}$(as can be seen in Fig. 1.24, that of $\mathrm{H}_{3}^{+}$is about 10 times smaller), it is safe to assume that $\mathrm{H}_{5}^{+}$dominates the recombination inside the cavity.

3.2.1 Recombination Uncertainties. The error in the equilibrium method for calculating $\beta$ comes from the beam intensity measurement, the particle number calculation, and the effective plasma volume that was obtained via simulation. The beam intensity error is the same as described in Sec.3.1.1. To calculate the number of electron-ion pairs made, the gas power and energy loss from Sec. 3.1 are used, and so any error in the energy loss is carried over. At equilibrium, the klystron is providing almost all of the power to the gas $(d V / d t \approx 0)$, and so the resistance of the cavity contributes. This comes from the capacitance (see Sec. 3.1.1) and the time constant from the fit to the cavity voltage decay. These are fairly consistent, although the resistance of the cavity varied over the range $\approx 1-2.5 \mathrm{M} \Omega$ over the duration of the experiment. The error from the effective plasma volume comes from knowing the distribution of electrons and ions compared to the distribution of the electric field in the cavity. If there is a longer tail of electrons distributed out near the edges of the cavity, this will change the effective electric field the plasma sees. Combined, these represent an error $(<50 \%)$ much smaller than the statistical error.

The error in the recursion method comes from fitting the calculated power to the measured power, and the simulated plasma distribution. The distribution evolves over time through ambipolar diffusion. Briefly, two oppositely charged particles experience an attraction due to Coulomb's law. In our case, these are electrons and 
hydrogen ions. When the electron moves away from the ion, it feels a force retarding its motion. Therefore it moves more slowly than it would otherwise. The larger the charge it sees, the more slowly it moves. Because of this, the plasma diffuses with roughly the mobility of the ions. So using the Einstein relation, $D=\mu \frac{k_{B} T}{e}$, and with our electron energy range of $k_{B} T=0.133-1.33 \mathrm{eV}$, and using $\mu=9.6 \frac{\mathrm{cm}^{2}}{\mathrm{~V}}$, the rate of diffusion is $1.28-12.6 \times 10^{-6} \frac{\mathrm{cm}^{2}}{\mu \mathrm{S}}$, meaning the distribution changes only slightly with time (the volume of the cavity is approximately $3000 \mathrm{~cm}^{3}$ ).

The error involved in fitting the power curves is highly pressure and beam intensity dependent. This can partially be seen in Fig. 3.30, in which the the time that recombination begins goes from $\approx 1 \mu$ s at 300 psi to $<0.5 \mu$ s at 1470 psi. This difference is even greater at low beam intensities. What this means is that for the 300 psi case, the value of $\beta$ that is fed into the recursion formula does not change the shape of the resulting power curve for the first microsecond. After this time, the value of $\beta$ will change the shape of the tail of the power curve. As the pressure increases and recombination begins earlier, more control over the initial shape of the power curve is gained by varying $\beta$. Because of this, large changes in $\beta$ have little effect on fitting the majority of the curves at $300 \mathrm{psi}$ and low beam intensity, but a much greater effect at $1520 \mathrm{psi}$ and high beam intensity. Additionally, in some cases the calculated power curve would not match the measured curve over the entire beam pulse, i.e., too much power might be calculated while the cavity is dumping its energy into the gas, while too little power is calculated in the equilibrium region, indicating either the energy loss or functional form of $\beta$ inputs were inaccurate. Table 3.5 summarizes the errors on $\beta$.

Because of the large errors associated with this measurement, an order of magnitude estimation of the recombination rate seems appropriate. The data clearly suggest that the effective $\beta$ that we measure is mostly due to $\mathrm{H}_{5}^{+}$, on the order of 
Table 3.5. Errors on $\beta$.

\begin{tabular}{lll}
\hline \hline Source & Affects & Estimate (\%) \\
\hline$d w$ & Calculation of $n$ & 20 \\
Capacitance & Cavity resistance & 3 \\
Decay time constant & Cavity resistance & 3 \\
Plasma volume simulation & $\beta$ & 20 \\
Diffusion & Plasma volume & 0.1 \\
Gas pressure & Time recombination begins & 20 \\
\hline
\end{tabular}

$10^{-6} \frac{\mathrm{cm}^{3}}{\mathrm{~s}}$, and decreases with increasing $X$. A small beam intensity effect (roughly a factor of two over a factor of 10 in beam intensity) is observed. However, as will be seen in the next section, the effect of recombination is small compared to that of attachment when an electronegative gas is added to the cavity, and therefore not significant when considering the use of an HPRF cavity in a muon cooling-channel.

\subsection{Attachment}

For dry air-doped hydrogen there are three unique rate equations, one for each of $e^{-}, \mathrm{H}_{5}^{+}$(assuming all of the positive ions are $\mathrm{H}_{5}^{+}$), and $\mathrm{O}_{2}^{-}$:

$$
\begin{aligned}
\frac{d n_{e}}{d t} & =\dot{n}-\beta n_{e} n_{\mathrm{H}_{5}^{+}}-\frac{n_{e}}{\tau} \\
\frac{d n_{\mathrm{H}_{5}^{+}}}{d t} & =\dot{n}-\beta n_{e} n_{\mathrm{H}_{5}^{+}}-\eta n_{\mathrm{H}_{5}^{+}} n_{\mathrm{O}_{2}^{-}} \\
\frac{d n_{\mathrm{O}_{2}^{-}}^{-}}{d t} & =\frac{n_{e}}{\tau}-\eta n_{\mathrm{H}_{5}^{+}} n_{\mathrm{O}_{2}^{-}}
\end{aligned}
$$

where $\tau$ is the average lifetime of an electron before it gets attached to $\mathrm{O}_{2}$, and $\eta$ is the ion-ion recombination rate. The last term in the electron rate equation defines $\tau$ 
as an effective lifetime and can be written as:

$$
\begin{aligned}
\frac{n_{e}}{\tau} & =\sum_{m} k_{m} n_{e} n_{\mathrm{O}_{2}} n_{m} \\
\tau & =\frac{1}{\sum_{m} k_{m} n_{\mathrm{O}_{2}} n_{m}}
\end{aligned}
$$

where for our case, particle $m$ is one of $\mathrm{H}_{2}, \mathrm{O}_{2}$, or $N_{2}$. Because the concentration of dry air is so low, the dominant term is always that of hydrogen. Assuming attachment coefficients from Tab. 1.5, the closest the oxygen or nitrogen terms come to that of hydrogen is at $300 \mathrm{psi}$ and $5 \% \mathrm{DA}: k_{\mathrm{H}_{2}} n_{\mathrm{H}_{2}}=9.54 \times 10^{-11} \frac{\mathrm{cm}^{3}}{\mathrm{~s}}, k_{\mathrm{O}_{2}} n_{\mathrm{O}_{2}}=1.26 \times$ $10^{-11} \frac{\mathrm{cm}^{3}}{\mathrm{~s}}$, and $k_{N_{2}} n_{N_{2}}=0.321 \times 10^{-11} \frac{\mathrm{cm}^{3}}{\mathrm{~s}}$, and so oxygen contributes at most $13 \%$ of the total attachment rate. Figure 3.32 shows the predicted lifetimes of $\tau$ vs. DA concentration for four total pressures based on these numbers.

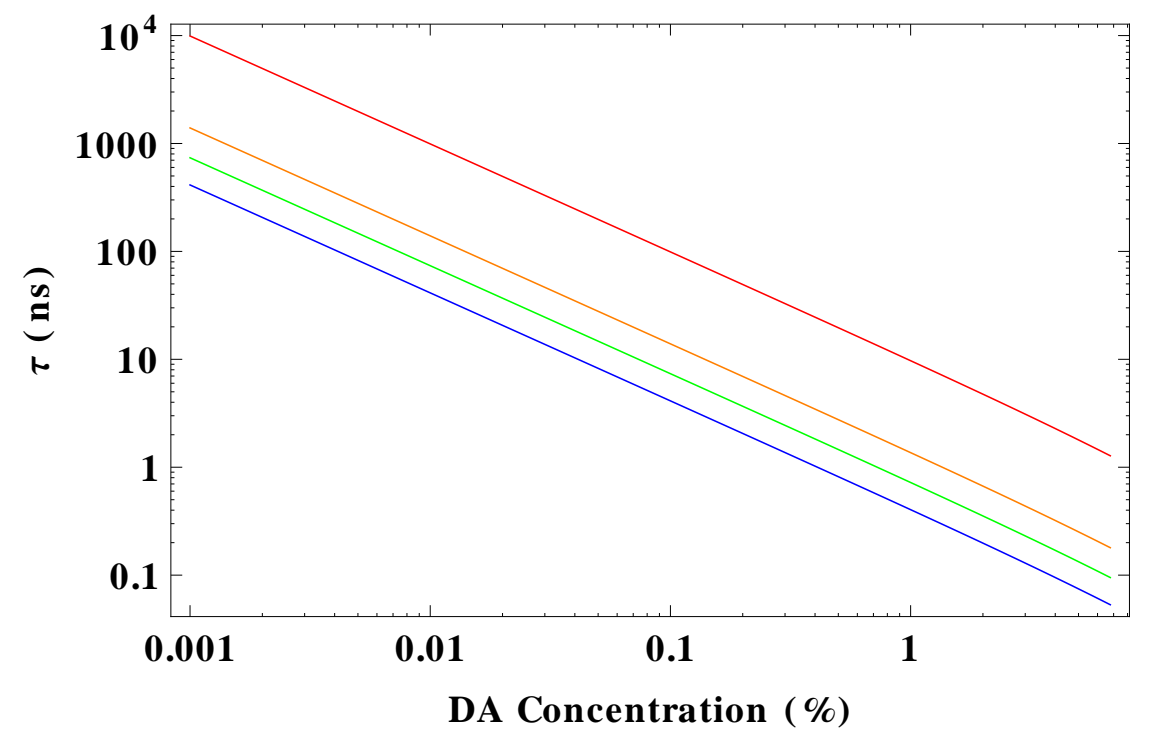

Figure 3.32. Prediction of $\tau$ based on Tab. 1.5 vs. DA concentration. Four pressures are plotted: 300 psi (red), 800 psi (orange), 1100 psi (green), and 1470 psi (blue).

A direct measurement of the attachment time or coefficient cannot be made. What can be done is to model the time evolution of the number of charged particles in the cavity with a recursion formula similar to that in Sec. 3.2 and use it to estimate 
the attachment time:

$$
\begin{aligned}
N_{e}(s+1)= & N_{e}(s)+\dot{N}(t(s)) \delta t-\beta(V(t(s))) N_{e}(s) N_{\mathrm{H}_{5}^{+}}(s) \delta t \\
& -\frac{N_{e}(s)}{\tau(V(t(s)))} \delta t \\
N_{\mathrm{H}_{5}^{+}}(s+1)= & N_{\mathrm{H}_{5}^{+}}(s)+\dot{N}(t(s)) \delta t-\beta(V(t(s))) N_{e}(s) N_{\mathrm{H}_{5}^{+}}(s) \delta t \\
& -\eta(V(t(s))) N_{\mathrm{H}_{5}^{+}}(s) N_{\mathrm{O}_{2}^{-}}(s) \delta t \\
N_{\mathrm{O}_{2}^{-}}(s+1)= & N_{\mathrm{O}_{2}^{-}}(s)+\frac{N_{e}(s)}{\tau(V(t(s)))} \delta t-\eta(V(t(s))) N_{\mathrm{H}_{5}^{+}}(s) N_{\mathrm{O}_{2}^{-}}(s) \delta t(3.2) \quad N_{\mathrm{H}_{5}^{+}}(1)=0, \quad N_{\mathrm{O}_{2}^{-}}(1)=0 \\
t(s+1)= & t(s)+\frac{1}{2 f} \quad t_{0}, \quad N_{e}(1)=0, \quad
\end{aligned}
$$

where one can see that the electron attachment time as a function of voltage $\tau(V(t))$ and the ion-ion recombination rate as a function of voltage $\eta(V(t))$ have been added due to the presence of oxygen. Here, functions dependent on voltage for $\tau(\tau(V)=$ $\left.a_{1} V^{a_{2}}\right)$ and $\eta\left(\eta(V)=b_{1} V^{b_{2}}\right)$ are fed into the recursion formula, where $a_{1}, a_{2}, b_{1}$, and $b_{2}$ are used as the fitting parameters. The measured energy loss of electronhydrogen pairs in addition to the mobilities for $\mathrm{H}_{5}^{+}$and $\mathrm{O}_{2}^{-}$given in Tab. 1.4 are used to calculate the power loss in the cavity. The value of $\tau$ is used to fit the beginning of the power curve, where the majority of power consumption comes from electrons, and the value of $\eta$ is used to fit the end of the power curve, where the majority of power consumption comes from ions. Because this method relies on $d w$ and $\beta$ as inputs, only pressures for which pure hydrogen data were recorded $(300,800,1100$ and $1470 \mathrm{psi}$ ) could be analyzed.

Figures 3.33 and 3.34 show two extremes, the power fits for 300 psi and 1470 psi. The plots on the left are the unaveraged data, which much be used to fit the beginning of the power curves, as averaging (over time) smooths out the start of the beam. The plots on the right are the data averaged over $90 \mathrm{~ns}$, which can be seen to benefit fitting the end of the power curves (where the power isn't changing much and the fitting 
isn't hurt by averaging).
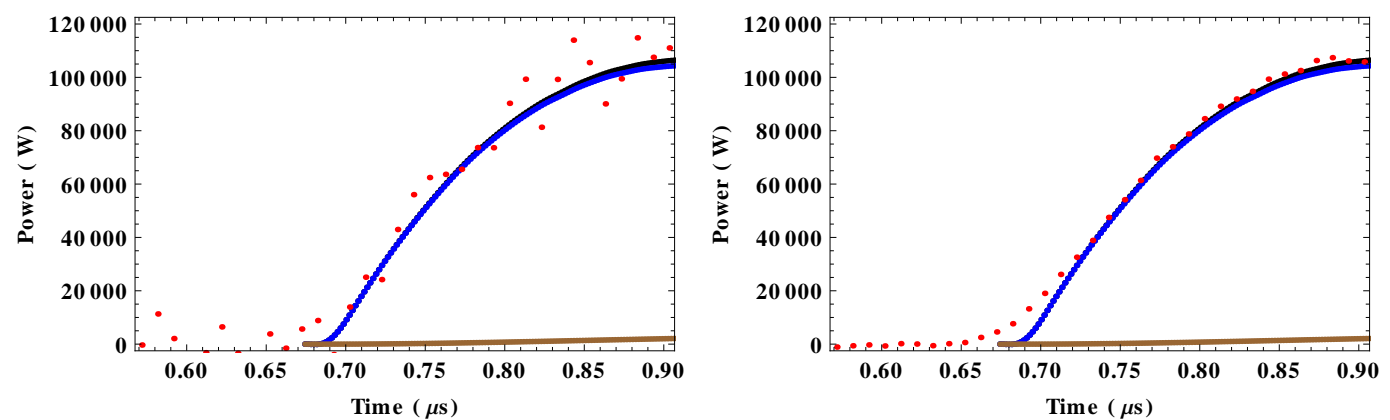

(a). Beginning of the unaveraged power curve.

(b). Beginning of the averaged power

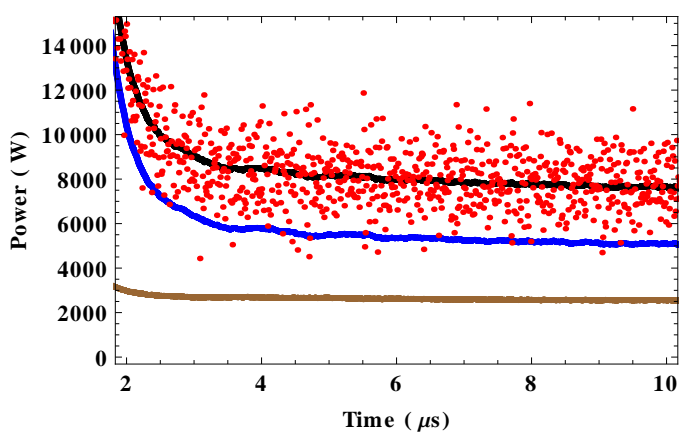
curve.

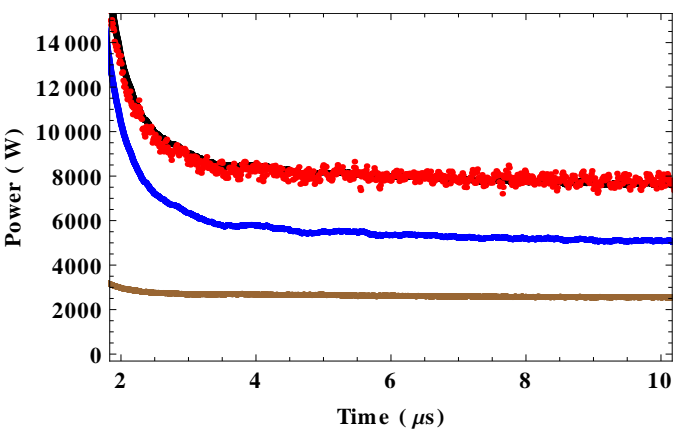

(c). End of the unaveraged power curve. (d). End of the averaged power curve.

Figure 3.33. Fitted power curves for 300 psi hydrogen doped with $0.04 \%$ dry air (18 MV $/ \mathrm{m}$, full beam intensity). The measured power is in red, the total calculated power is in black, the contribution from electrons is in blue, and the contribution from ions is in brown. The plots show fits to the beginning (top) and end (bottom) of unaveraged (left) and averaged (right) data, where the average is taken over $90 \mathrm{~ns}$ time bins. 

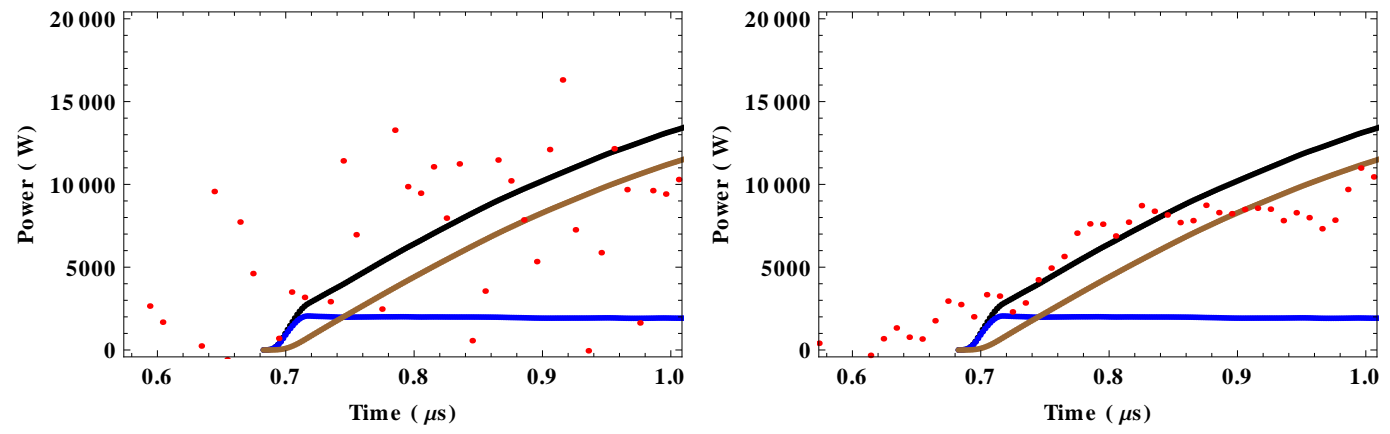

(a). Fit to the unaveraged beginning of (b). Fit to the averaged beginning of the the power curve.

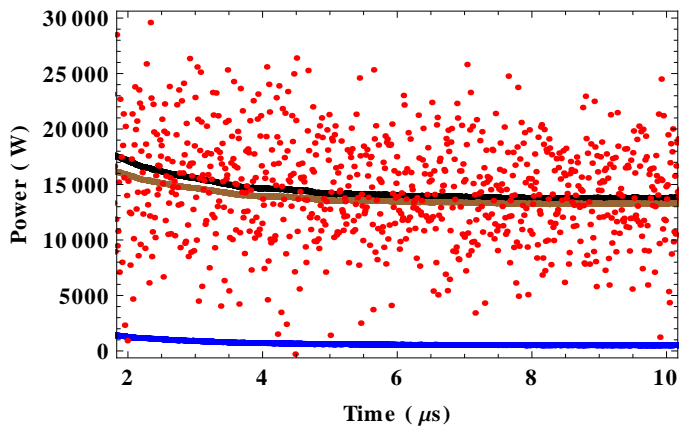
power curve.

(c). Fit to the unaveraged end of the (d). Fit to the averaged end of the power power curve.

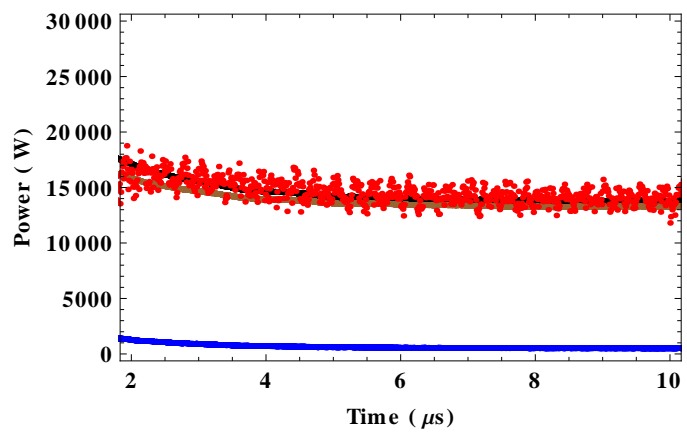

Figure 3.34. Fitted power curves for 1470 psi hydrogen doped with $1 \%$ dry air (20 MV/m, full beam intensity). The measured power is in red, the total calculated power is in black, the contribution from electrons is in blue, and the contribution from ions is in brown. The left plots show the unaveraged data, and the right plots show the data averaged over 90 ns time bins. 
As can be seen in Fig. 3.33, for 300 psi, at the start of the beam all of the energy loss comes from electrons, while at the end of the beam pulse the ions begin to contribute. For 1470 psi in Fig. 3.34, the electrons only contribute a small amount to the energy loss at the start of the beam, while at the end the energy loss is entirely from ions. Figure 3.35 shows the results of the resursion formula for 300 and 1470 psi. The plots on the left show the number of particles in the cavity over the entire beam pulse, and the plots on the right show the averaged power over the entire beam pulse.

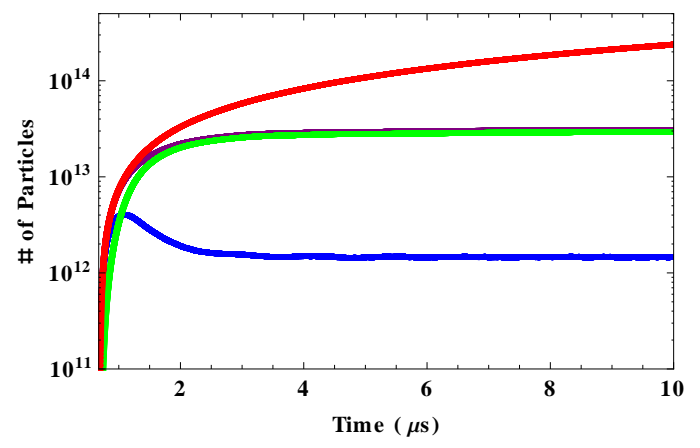

(a). Number of particles in the cavity at 300 psi.

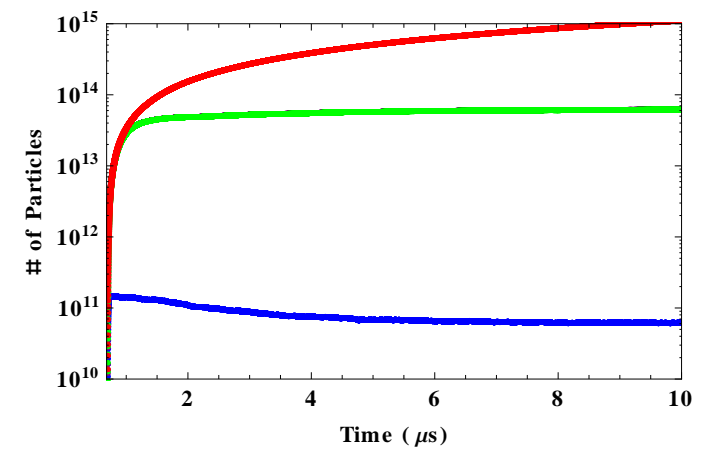

(c). Number of particles in the cavity at 1470 psi.

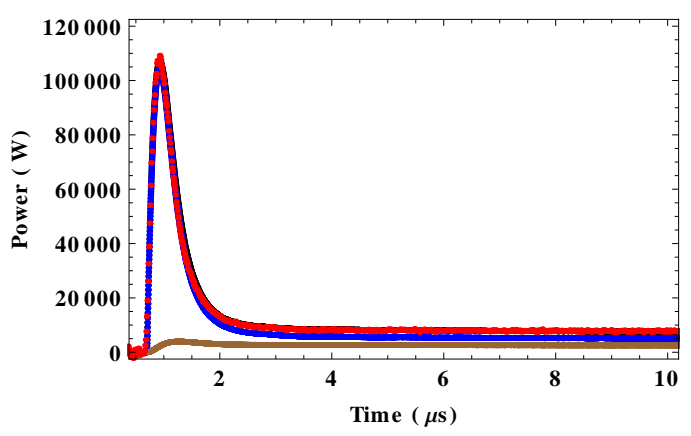

(b). Averaged power for 300 psi.

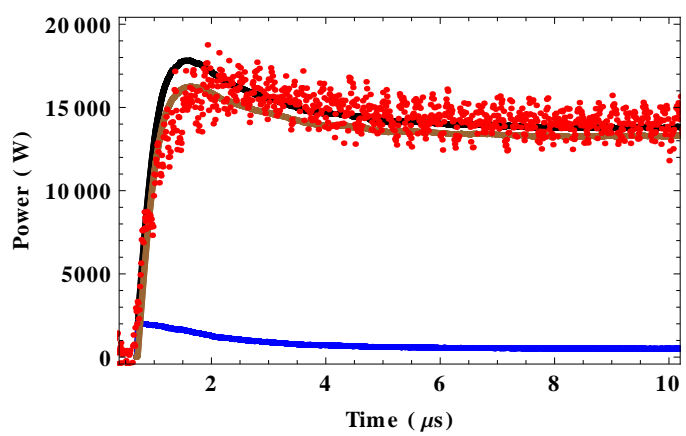

(d). Averaged power for 1470 psi.

Figure 3.35. Results of the recursion formula for dry air-doped hydrogen. The plots on the top are $300 \mathrm{psi}$ and the bottom are $1470 \mathrm{psi}$. The plots on the left are the number of particles in the cavity $\left(e^{-}-\right.$blue, $\mathrm{H}_{5}^{+}-$purple, $\mathrm{O}_{2}^{-}-$green, and total produced - red) vs. time. The plots on the right are the averaged power vs. time. The measured power is in red, the total calculated power is in black, the contribution from electrons is in blue, and the contribution from ions is in brown. 
If one compares Fig. 3.30a with Fig. 3.35a and Fig. 3.30c with Fig. 3.35c, the number of electrons in the cavity is significantly reduced when hydrogen is doped with dry air. The fitting method described here gives as a result $\tau$ and $\eta$ as functions of $V$, so the initial voltage in the cavity was selected with the corresponding value of $\tau$ or $\eta$ to be plotted in the following figures in order to cover a wider range of $X$.

The resulting values of $\tau$ vs. DA concentration and $\mathrm{O}_{2}$ number density are shown in Figs. 3.36 and 3.37. The data from [89] (Fig. 1.27) on oxygen doped hydrogen was used to predict the attachment time at $1.88 \%$ concentration (the value used in that experiment); the intersection of the horizontal and vertical lines on the plots is that point. The dashed line is the prediction based on Eq.3.23 and the rate coefficients from Tab. $1.5\left(k_{\mathrm{H}_{2}}=4.8 \times 10^{-31} \frac{\mathrm{cm}^{6}}{\mathrm{~s}}, k_{\mathrm{O}_{2}}=2.5 \times 10^{-30} \frac{\mathrm{cm}^{6}}{\mathrm{~s}}\right.$, and $k_{N_{2}}=$ $\left.1.6 \times 10^{-31} \frac{\mathrm{cm}^{6}}{\mathrm{~s}}\right)$. 


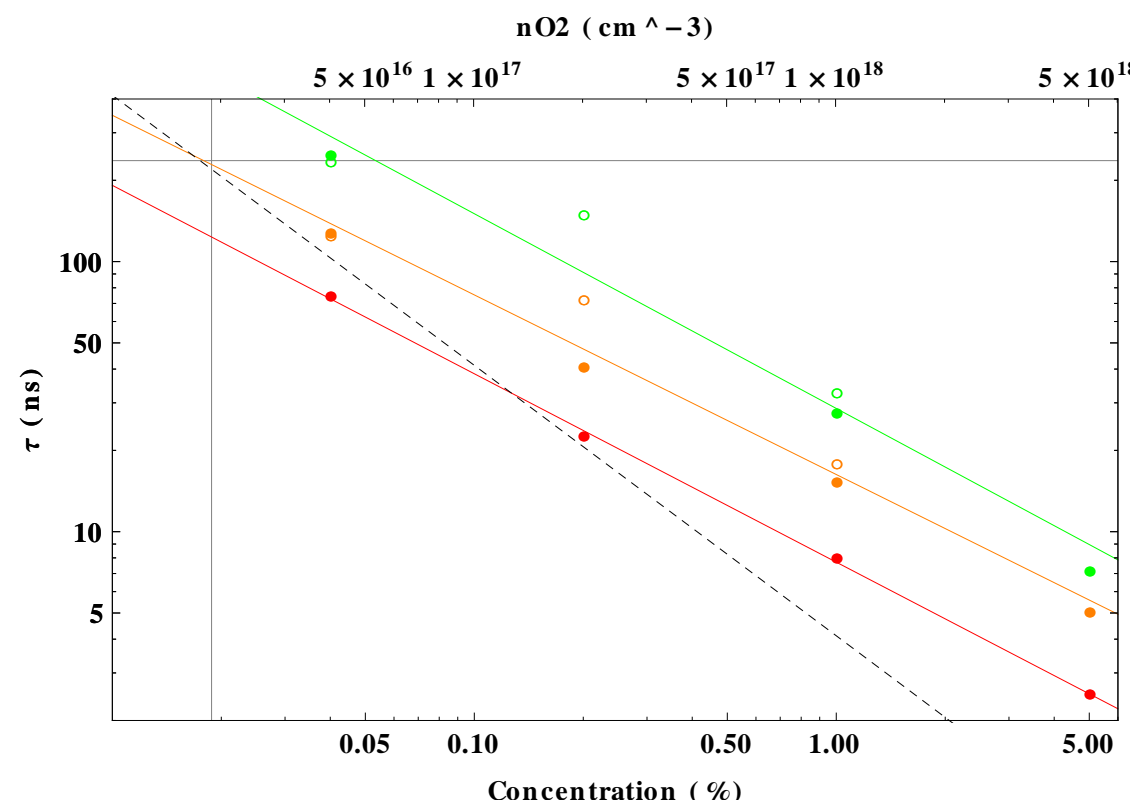

(a). $300 \mathrm{psi} ; 5 \mathrm{MV} / \mathrm{m}$ (red), $10 \mathrm{MV} / \mathrm{m}$ (orange), $18 \mathrm{MV} / \mathrm{m}$ (green).

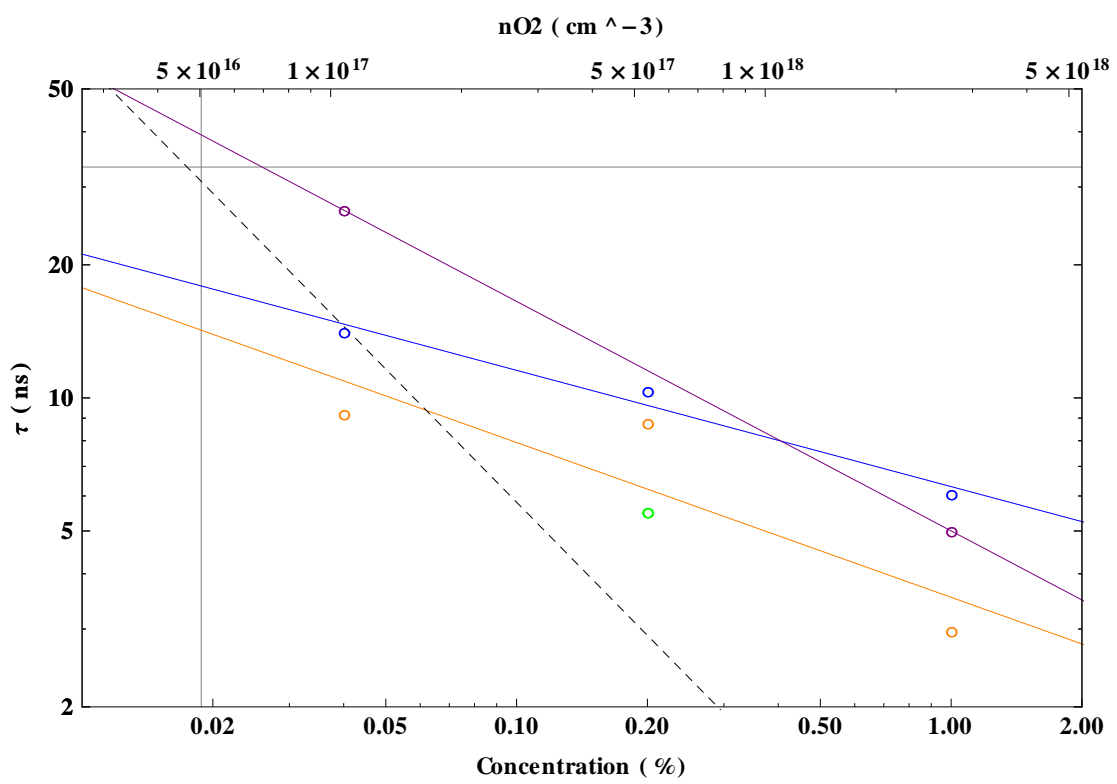

(b). $800 \mathrm{psi} ; 5 \mathrm{MV} / \mathrm{m}$ (red), $10 \mathrm{MV} / \mathrm{m}$ (orange), $20 \mathrm{MV} / \mathrm{m}$ (green), $25 \mathrm{MV} / \mathrm{m}$ (blue).

Figure 3.36. Attachment times vs. DA concentration and $\mathrm{O}_{2}$ density. The filled points are high beam intensity and the empty points are low beam intensity. The solid colored lines are fits to the data and the dashed black line is a prediction based on the attachment coefficients in Tab. 1.5. The intersection of the horizontal and vertical lines is the prediction based on data from [89]. 


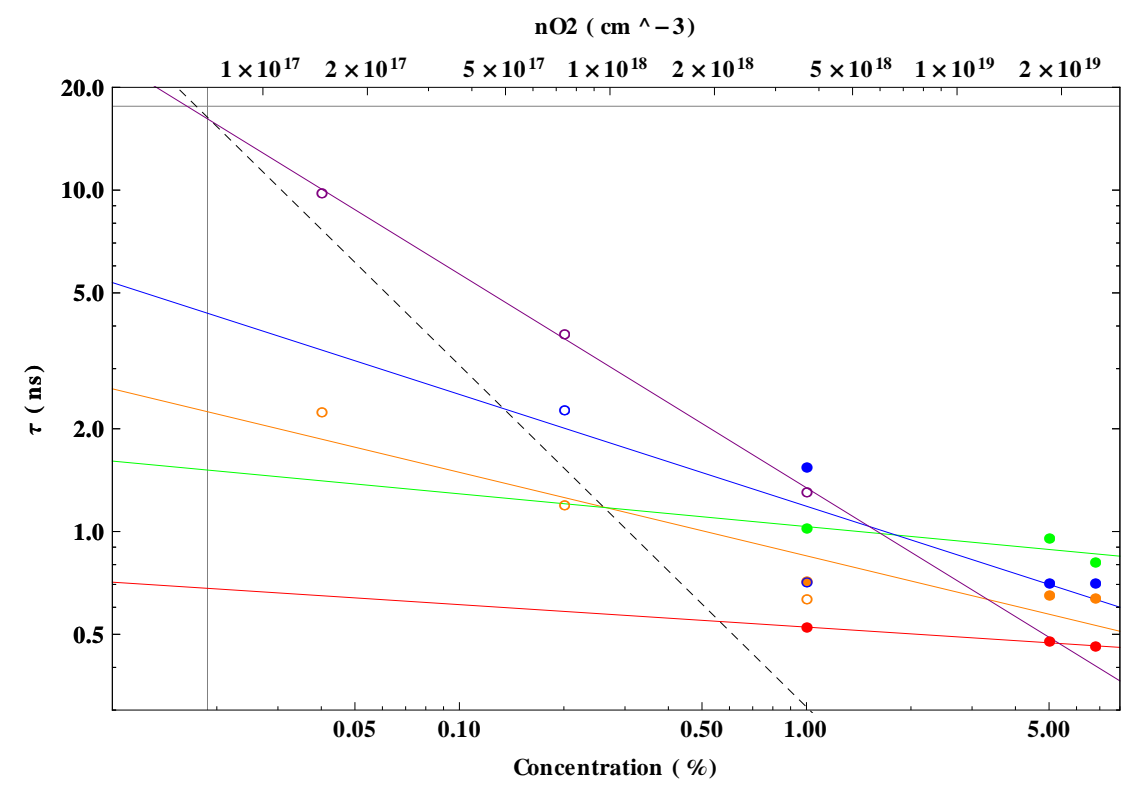

(a). $1100 \mathrm{psi} ; 5 \mathrm{MV} / \mathrm{m}$ (red), $10 \mathrm{MV} / \mathrm{m}$ (orange), $20 \mathrm{MV} / \mathrm{m}$ (green), $25 \mathrm{MV} / \mathrm{m}$ (blue).

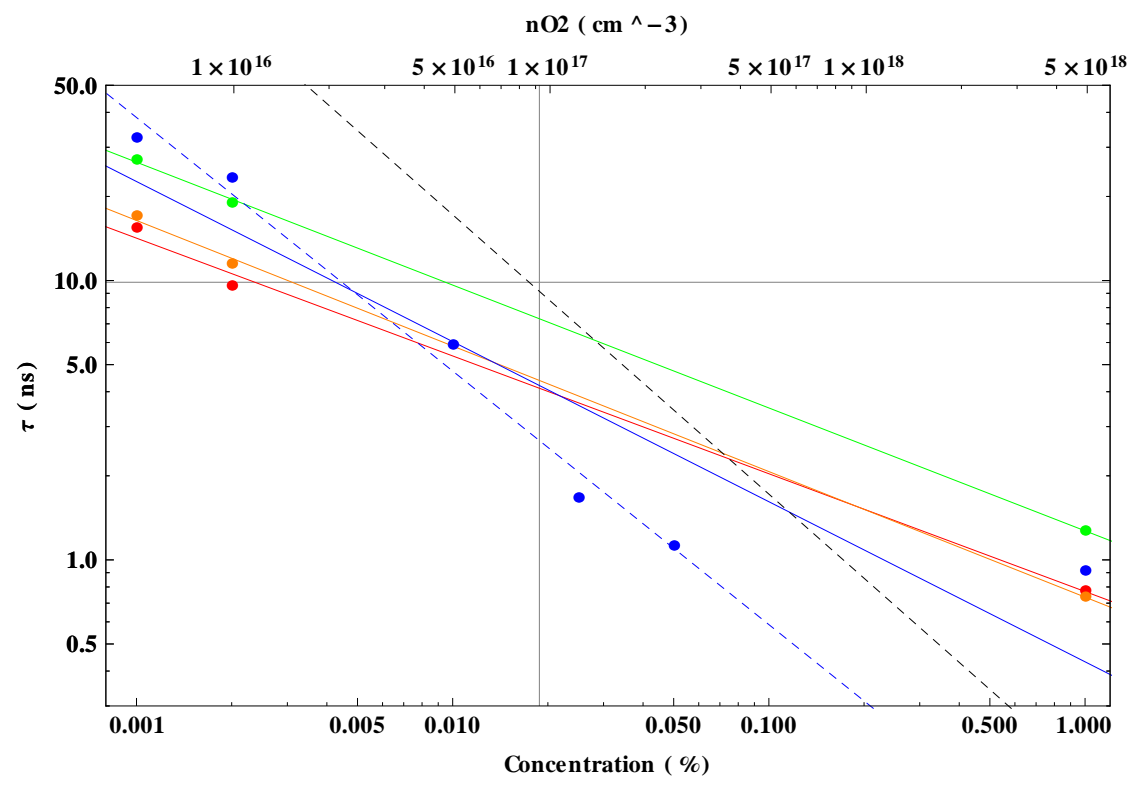

(b). $1470 \mathrm{psi} ; 5 \mathrm{MV} / \mathrm{m}$ (red), $10 \mathrm{MV} / \mathrm{m}$ (orange), $20 \mathrm{MV} / \mathrm{m}$ (green), $25 \mathrm{MV} / \mathrm{m}$ (blue).

Figure 3.37. Attachment times vs. DA concentration and $\mathrm{O}_{2}$ density. The filled points are high beam intensity and the empty points are low beam intensity. The solid colored lines are fits to the data and the dashed black line is a prediction based on the attachment coefficients in Tab. 1.5 (the dashed blue line in (b) is a fit that doesn't include the $1 \%$ point). The intersection of the horizontal and vertical lines is the prediction based on data from [89]. 
Notice that for all pressures, as the concentration of DA increases, the lifetime of electrons in the cavity decreases. Assuming a constant attachment coefficient, $\tau \propto 1 / n_{\mathrm{O}_{2}}$ (if the number of oxygen molecules is doubled, the collision frequency of electrons with oxygen should double, and therefore the attachment time will be halved). However, we observe $\tau \propto 1 / n_{\mathrm{O}_{2}}^{\alpha}$, where $\alpha \approx 0.4-0.7$. This indicates that the attachment coefficient depends upon the DA concentration. Figure 1.30 from [81] supports this result, in which the rate coefficient is seen to increase with increasing concentration of oxygen in helium at a constant $X$ (it is not explicitly stated that $P$ is constant in those measurements, but that is a relatively safe assumption). Recalling the relationship between the attachment time and rate coefficient:

$$
k=\frac{1}{\tau n_{\mathrm{O}_{2}} n_{m}}
$$

it can be inferred from those measurements that for $k$ to increase with increasing oxygen concentration (assuming a constant $n_{m}$ ), $\tau$ must decrease faster than $n_{\mathrm{O}_{2}}$ increases, or $\tau \propto 1 / n_{\mathrm{O}_{2}}^{\alpha}$, where $\alpha>1$. Our most reliable measurements of $\tau$ are at 300 psi, for which $\alpha \approx 0.7$. However, no measurements of the rate coefficient vs. oxygen concentration in hydrogen have been made, particularly in the pressure ranges for this experiment.

An important thing to note is that at higher pressures the front end of the power curve is more noisy (due to smaller changes in voltage), and so the fits are less accurate. Also, for small attachment times (below $1 \mathrm{~ns}$ ) the calculated values for $\tau$ are upper limits due to limitations in the algorithm used. This is evident for the 1100 psi data at large concentrations of DA. For this reason, the $1 \%$ data point at 1470 psi was dropped from one fit.

As shown in Fig. 1.27, the only previously reported data on attachment for oxygen doped hydrogen match our data set remarkably well, considering the attachment time was extrapolated $2-3$ orders of magnitude higher in hydrogen pressure. 
Unfortunately the energy of the electrons in that experiment was not reported (only that that they were excited with an RF pulse and the gas temperature was $290 \mathrm{~K}$ ). However this does seem to indicate that extrapolation to higher hydrogen pressure is valid.

Table 3.6 shows the fits for $\tau$ vs. DA concentration. 
Table 3.6. Fits of $\tau$ vs. concentration for DA doped hydrogen of the form $\tau=c_{1} C^{-c_{2}}$.

\begin{tabular}{|c|c|c|c|}
\hline Pressure (psi) & $\mathrm{E}(\mathrm{MV} / \mathrm{m})$ & $c_{1}\left(10^{-9}\right)$ & $c_{2}$ \\
\hline 300 & 5 & 7.713 & 0.698 \\
\hline 300 & 10 & 16.283 & 0.665 \\
\hline 300 & 18 & 28.600 & 0.721 \\
\hline 800 & 10 & 3.539 & 0.350 \\
\hline 800 & 25 & 6.300 & 0.263 \\
\hline 800 & 40 & 5.002 & 0.519 \\
\hline 1100 & 5 & 0.525 & 0.066 \\
\hline 1100 & 10 & 0.849 & 0.245 \\
\hline 1100 & 20 & 1.034 & 0.096 \\
\hline 1100 & 25 & 1.186 & 0.328 \\
\hline 1100 & 40 & 1.343 & 0.626 \\
\hline 1470 & 5 & 0.770 & 0.422 \\
\hline 1470 & 10 & 0.736 & 0.449 \\
\hline 1470 & 20 & 1.271 & 0.440 \\
\hline 1470 & 25 & 0.432 & 0.573 \\
\hline
\end{tabular}


Figures 3.38 and 3.39 show the attachment times as functions of $X$ and the average kinetic energy of the electrons (where the instantaneous kinetic energy has been averaged over a Maxwellian distribution and over an RF cycle) for different pressures. In these plots, colors correspond to concentrations of DA. The solid lines are fits to the data. The dashed lines are predictions based on analytical forms of the attachment coefficient vs. electron and gas temperature given in [82] (Eq. 1.41). Only the coefficients of oxygen and nitrogen are given, and so the coefficient of hydrogen is estimated to be three times that of nitrogen, based on the numbers in Tab. 1.5. 


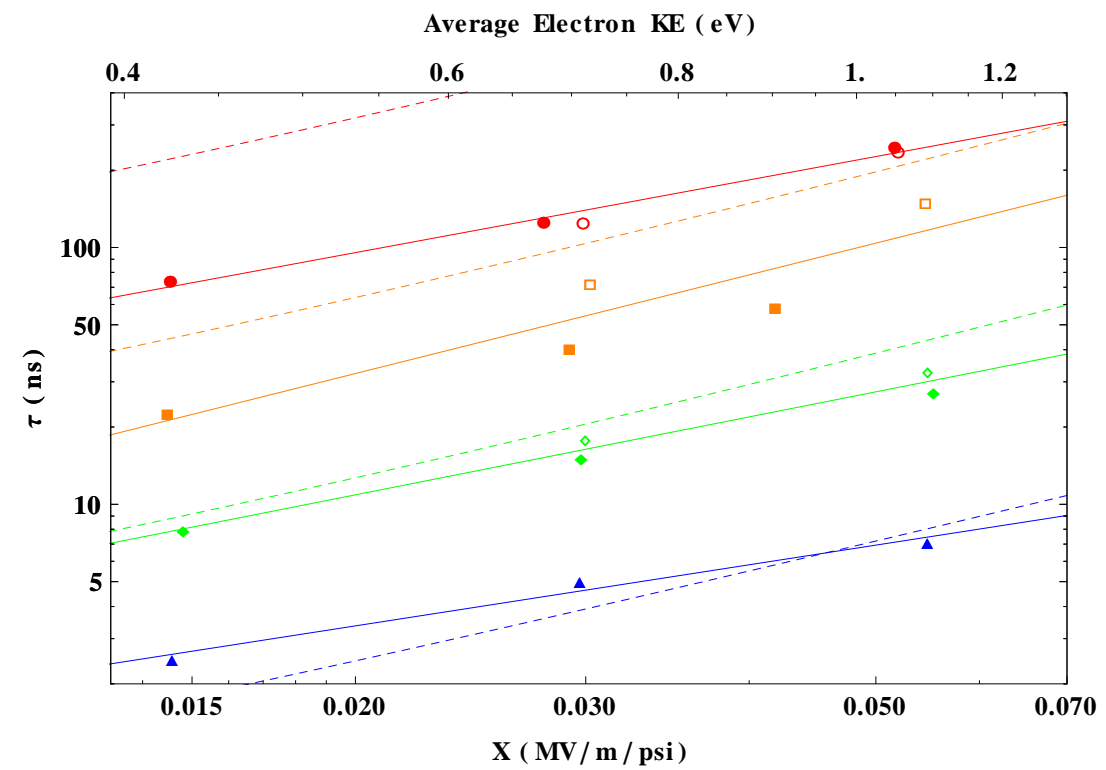

(a). 300 psi; $0.04 \%$ (red), $0.2 \%$ (orange), $1 \%$ (green), and $5 \%$ (blue).

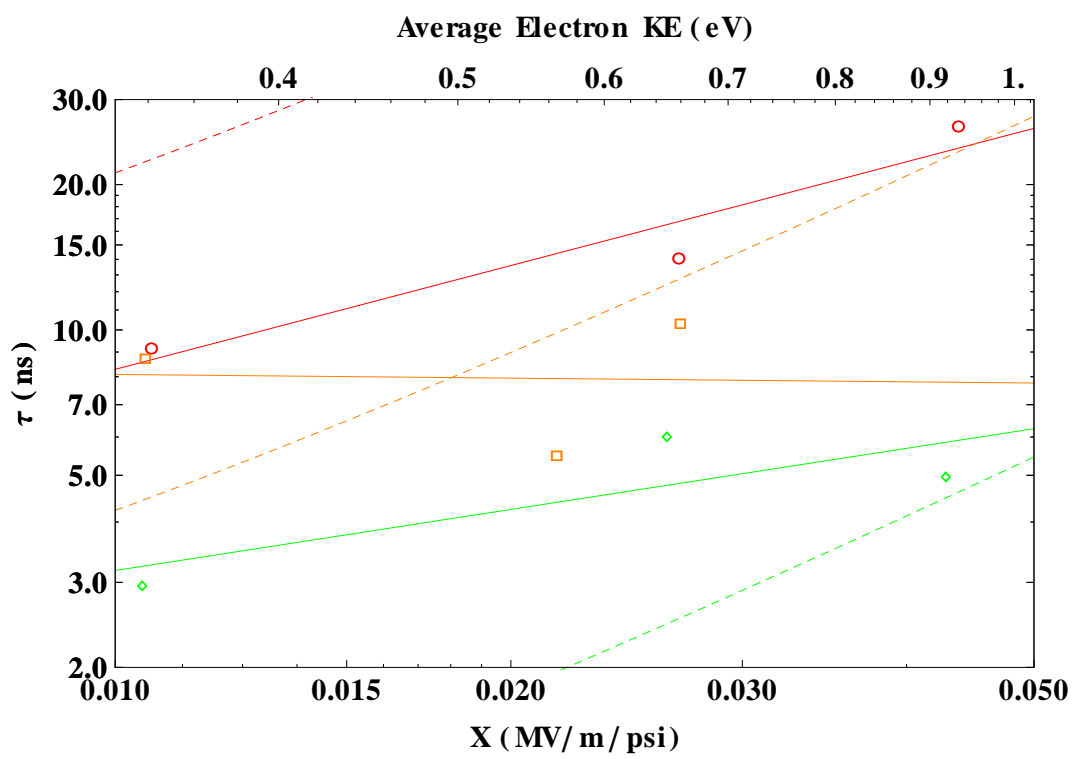

(b). $800 \mathrm{psi} ; 0.04 \%$ (red), $0.2 \%$ (orange), and $1 \%$ (green).

Figure 3.38. Attachment times vs. $X$ and average electron kinetic energy. The filled points are high beam intensity and the empty points are low beam intensity. The solid lines are fits to the data. The dashed lines are predictions based on Eq. 1.41. 


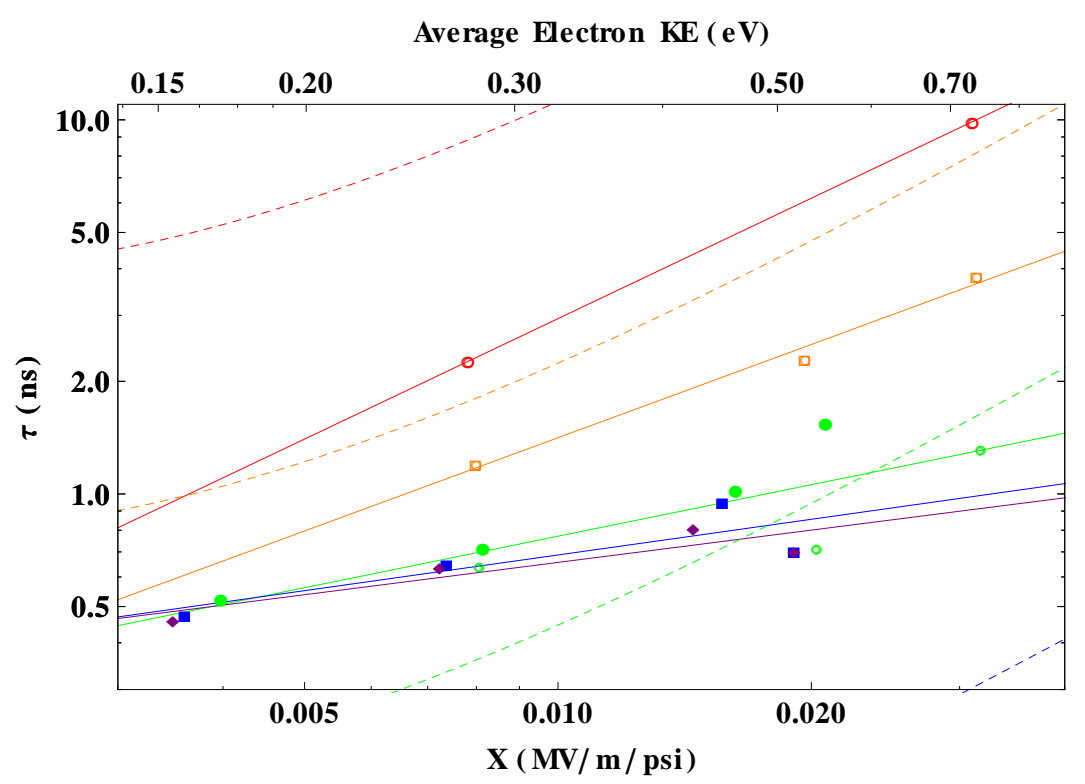

(a). $1100 \mathrm{psi} ; 0.04 \%$ (red), $0.2 \%$ (orange), $1 \%$ (green), $5 \%$ (blue), and $6.78 \%$ (purple).

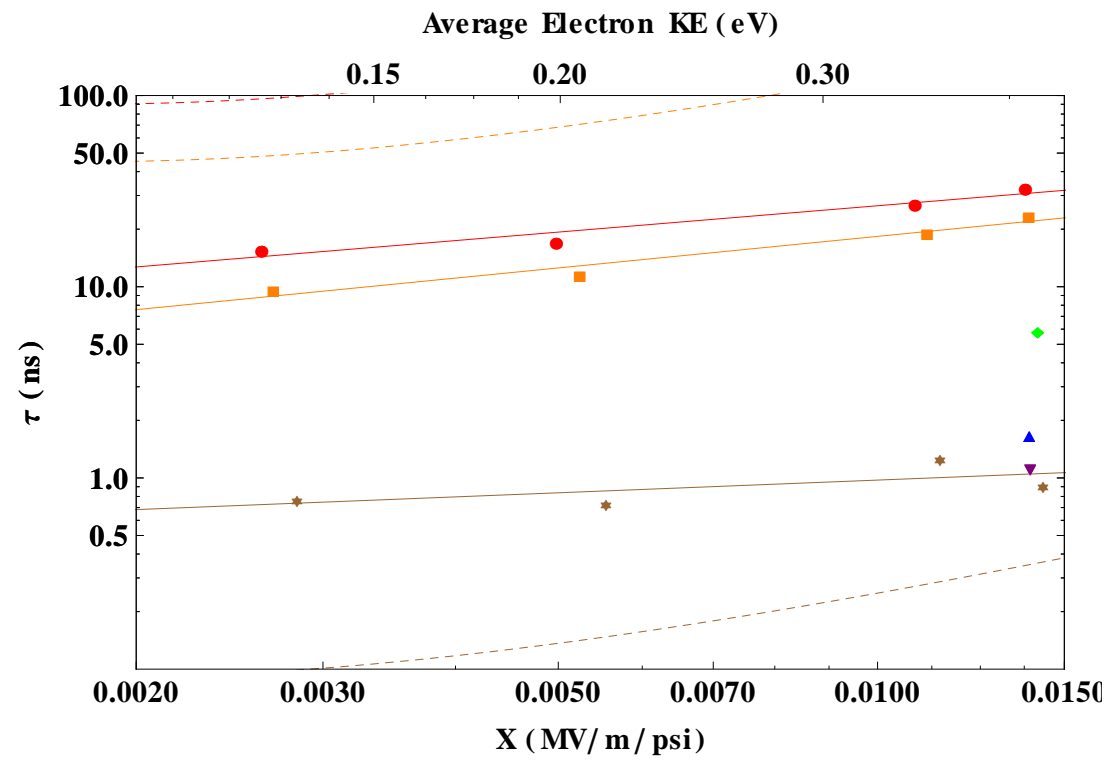

(b). 1470 psi; $0.001 \%$ (red), $0.002 \%$ (orange), $0.01 \%$ (green), $0.025 \%$ (blue), $0.05 \%$ (purple), and 1\% (brown).

Figure 3.39. Attachment times vs. $X$ and average electron kinetic energy. The filled points are high beam intensity and the empty points are low beam intensity. The solid lines are fits to the data. The dashed lines are predictions based on Eq. 1.41. 
Notice that as the electric field increases, the attachment time increases. There is a large error in the attachment time prediction, and so one would expect only order of magnitude agreement. Hydrogen is the dominant term in determining the attachment time, and the attachment coefficient was assumed to be three times that of nitrogen, based on the coefficients reported in Tab. 1.5. If the curves of nitrogen and oxygen are compared, one finds that the values of the attachment coefficient vary between factors of 10 and 50, and so hydrogen should not follow the electron temperature dependence of nitrogen exactly. Additionally, Eq.1.38 was produced using low pressure data, and there is clearly a pressure effect, as is evident by the discrepancy between prediction and measurement increasing with increasing pressure.

Table 3.7 shows the fits of $\tau$ vs. $X$. 
Table 3.7. Fits of the form $\tau=c_{1} X^{c_{2}}$ for DA doped hydrogen.

\begin{tabular}{|c|c|c|c|}
\hline Pressure (psi) & Concentration (\%) & $c_{1}\left(10^{-9}\right)$ & $c_{2}$ \\
\hline 300 & 0.04 & 3776 & 0.940 \\
\hline 300 & 0.2 & 4752 & 1.276 \\
\hline 300 & 1 & 561.1 & 1.008 \\
\hline 300 & 5 & 73.99 & 0.790 \\
\hline 800 & 0.04 & 222.0 & 0.714 \\
\hline 800 & 0.2 & 7.194 & -0.025 \\
\hline 800 & 1 & 21.99 & 0.420 \\
\hline 1100 & 0.04 & 404.8 & 1.069 \\
\hline 1100 & 0.2 & 64.04 & 0.828 \\
\hline 1100 & 1 & 6.331 & 0.457 \\
\hline 1100 & 5 & 2.954 & 0.317 \\
\hline 1100 & 6.78 & 2.458 & 0.287 \\
\hline 1470 & 0.001 & 218.4 & 0.458 \\
\hline 1470 & 0.002 & 229.2 & 0.548 \\
\hline 1470 & 1 & 2.692 & 0.221 \\
\hline
\end{tabular}


Figures 3.40, 3.41 and 3.42 show $\tau$ vs. total gas pressure for various electric fields. The colors correspond to concentration of DA. The solid lines are fits to the data, and the dashed line is the prediction based on [89] (Fig. 1.27). Note that the concentration of oxygen used in that experiment was $1.88 \%$. 


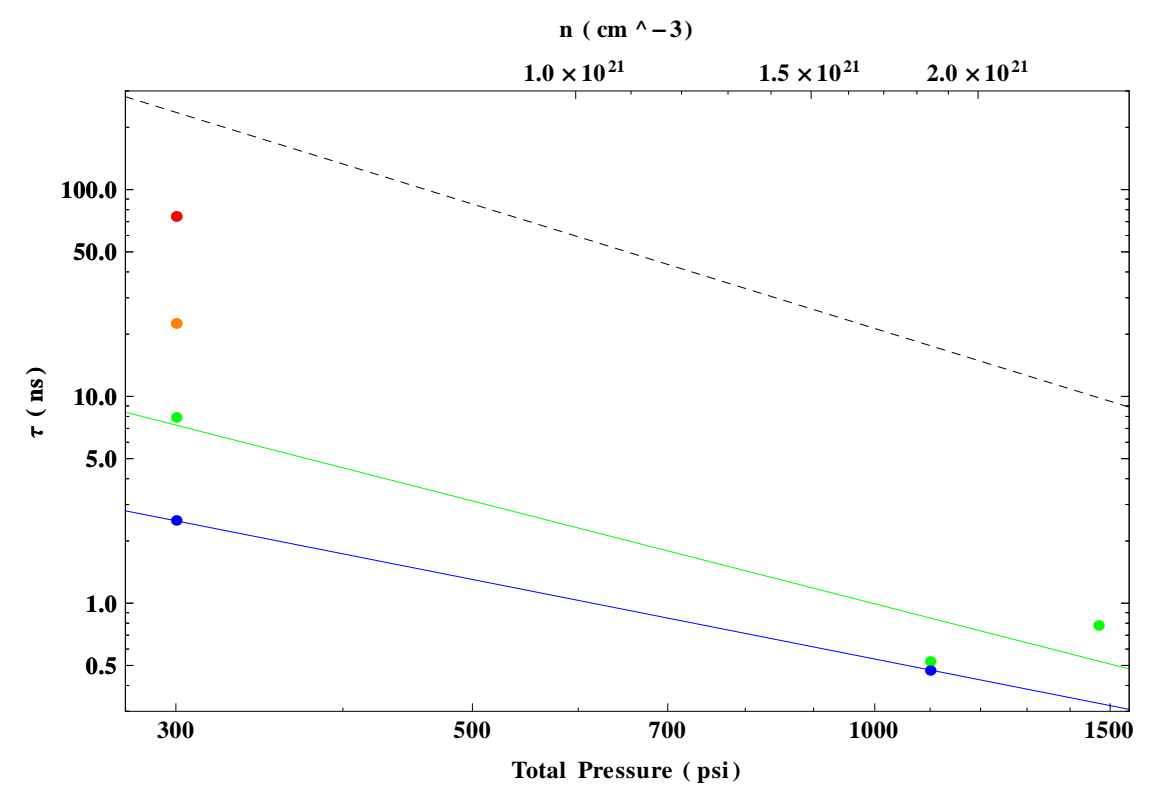

(a). $5 \mathrm{MV} / \mathrm{m} ; 0.04 \%$ (red), $0.2 \%$ (orange), $1 \%$ (green), and $5 \%$ (blue).

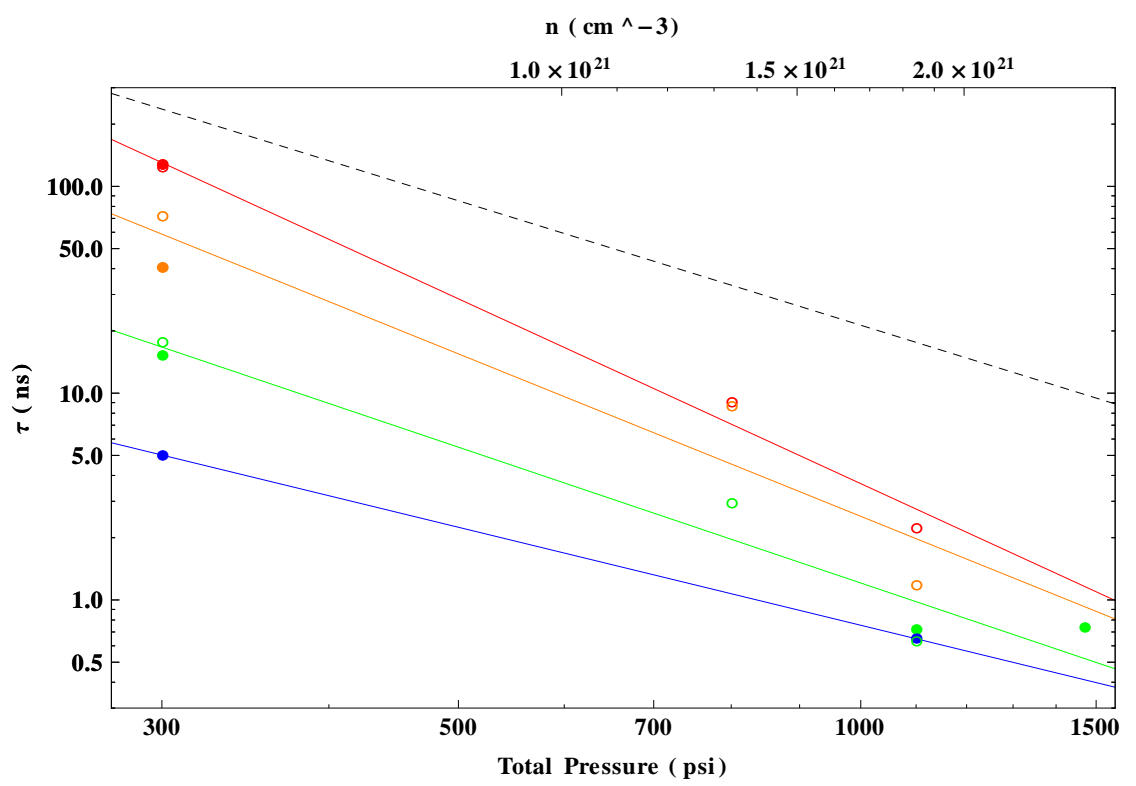

(b). $10 \mathrm{MV} / \mathrm{m} ; 0.04 \%$ (red), $0.2 \%$ (orange), $1 \%$ (green), and $5 \%$ (blue).

Figure 3.40. Attachment time vs. total pressure and number density. The filled points are high beam intensity and the empty points are low beam intensity. 


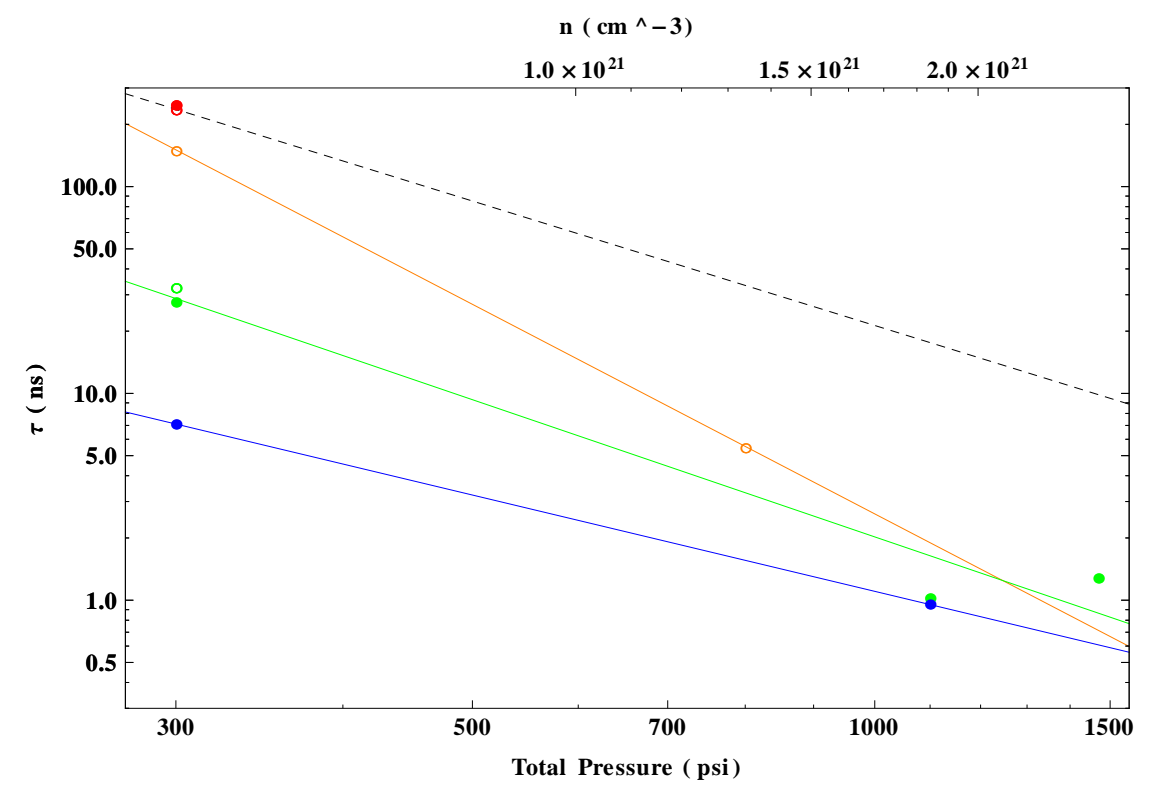

(a). $20 \mathrm{MV} / \mathrm{m} ; 0.04 \%$ (red), $0.2 \%$ (orange), $1 \%$ (green), and 5\% (blue).

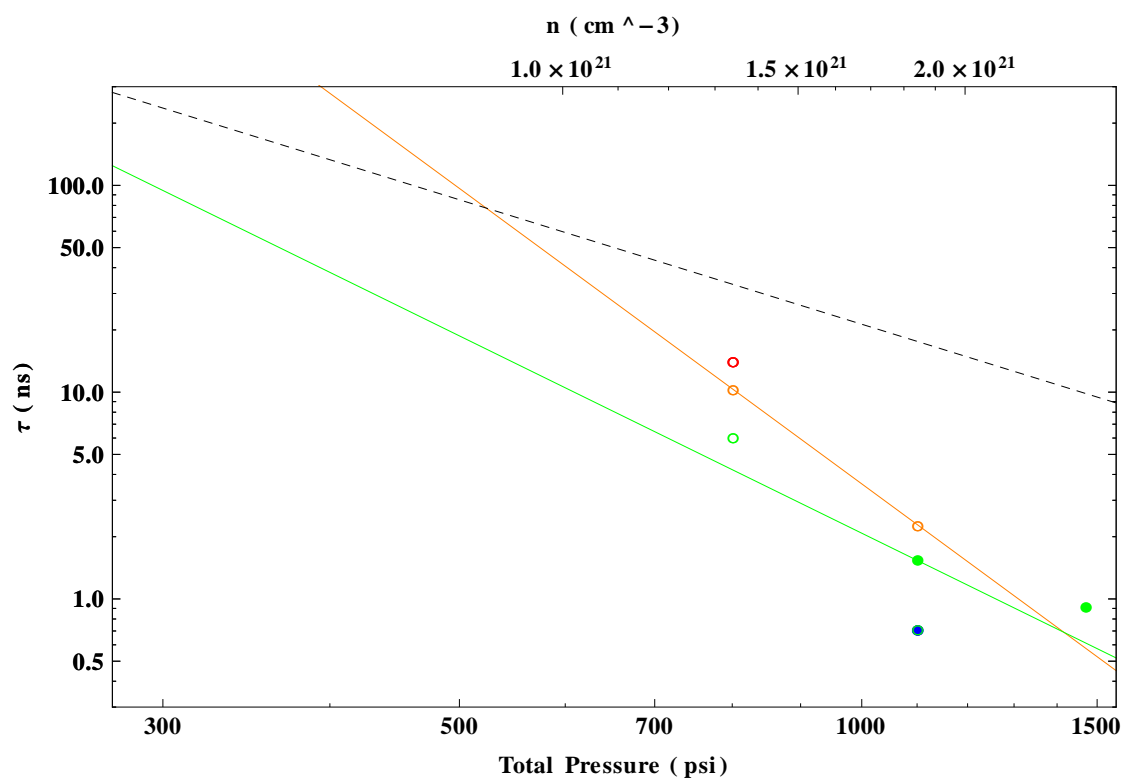

(b). $25 \mathrm{MV} / \mathrm{m} ; 0.04 \%$ (red), $0.2 \%$ (orange), $1 \%$ (green), and $5 \%$ (blue).

Figure 3.41. Attachment time vs. total pressure and number density. The filled points are high beam intensity and the empty points are low beam intensity. 


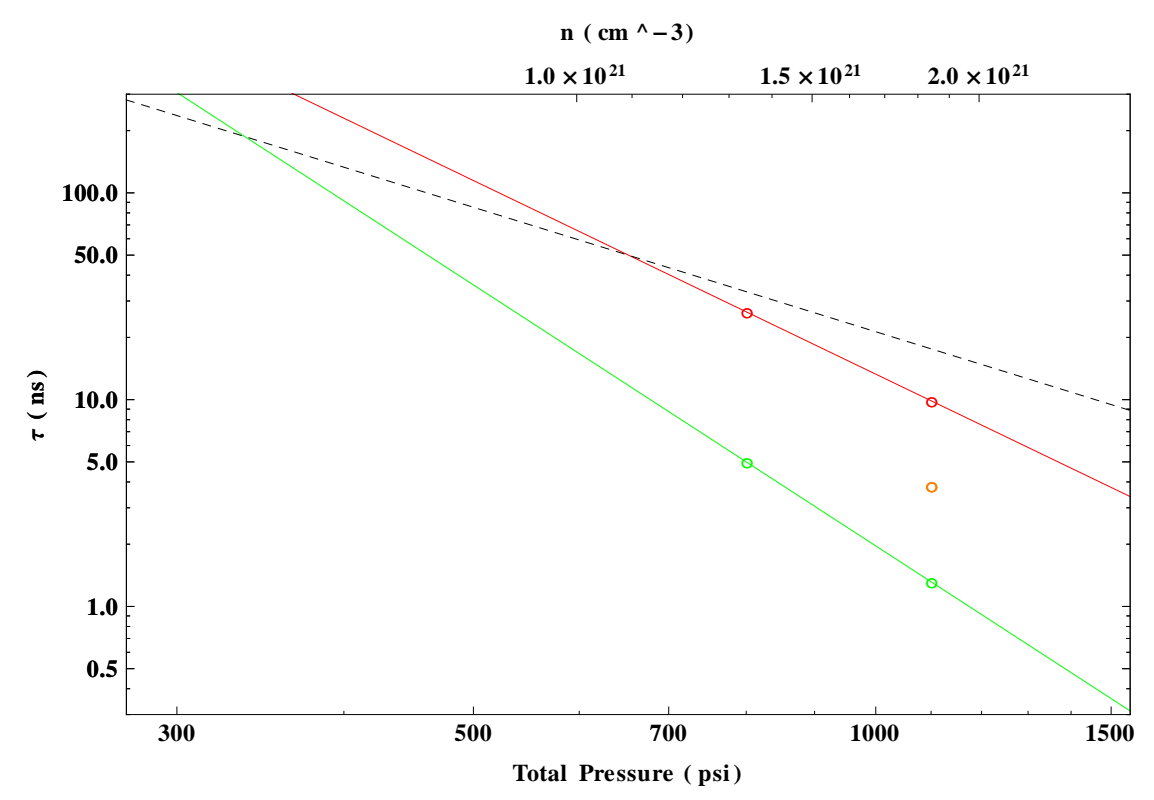

(a). $40 \mathrm{MV} / \mathrm{m} ; 0.04 \%$ (red), $0.2 \%$ (orange), $1 \%$ (green), and $5 \%$ (blue).

Figure 3.42. Attachment time vs. total pressure and number density. The filled points are high beam intensity and the empty points are low beam intensity.

Notice that as the total pressure increases, $\tau$ decreases. These plots represent an incomplete picture though, since, for a constant electric field, the average kinetic energy of an electron decreases with increasing gas pressure. Ideally, measurements would have been made at a constant electron kinetic energy. One would expect that at the same electron energy and oxygen concentration, the predicted value would coincide with the measurement. However, the energy of electrons in Ref. [89] is unknown.

Perhaps a more telling plot would be one of attachment time vs. total pressure for measurements made at constant electron kinetic energy. Unfortunately there are very few overlaps of kinetic energy over the range of pressures data were taken at, as can be seen in Fig. 3.43a. When $0.4<\epsilon<0.43 \mathrm{eV}$ is selected, Fig. 3.43b is the result. There are no overlaps in concentration or number density of oxygen for this data set. The dashed line is again the prediction from [89]. 


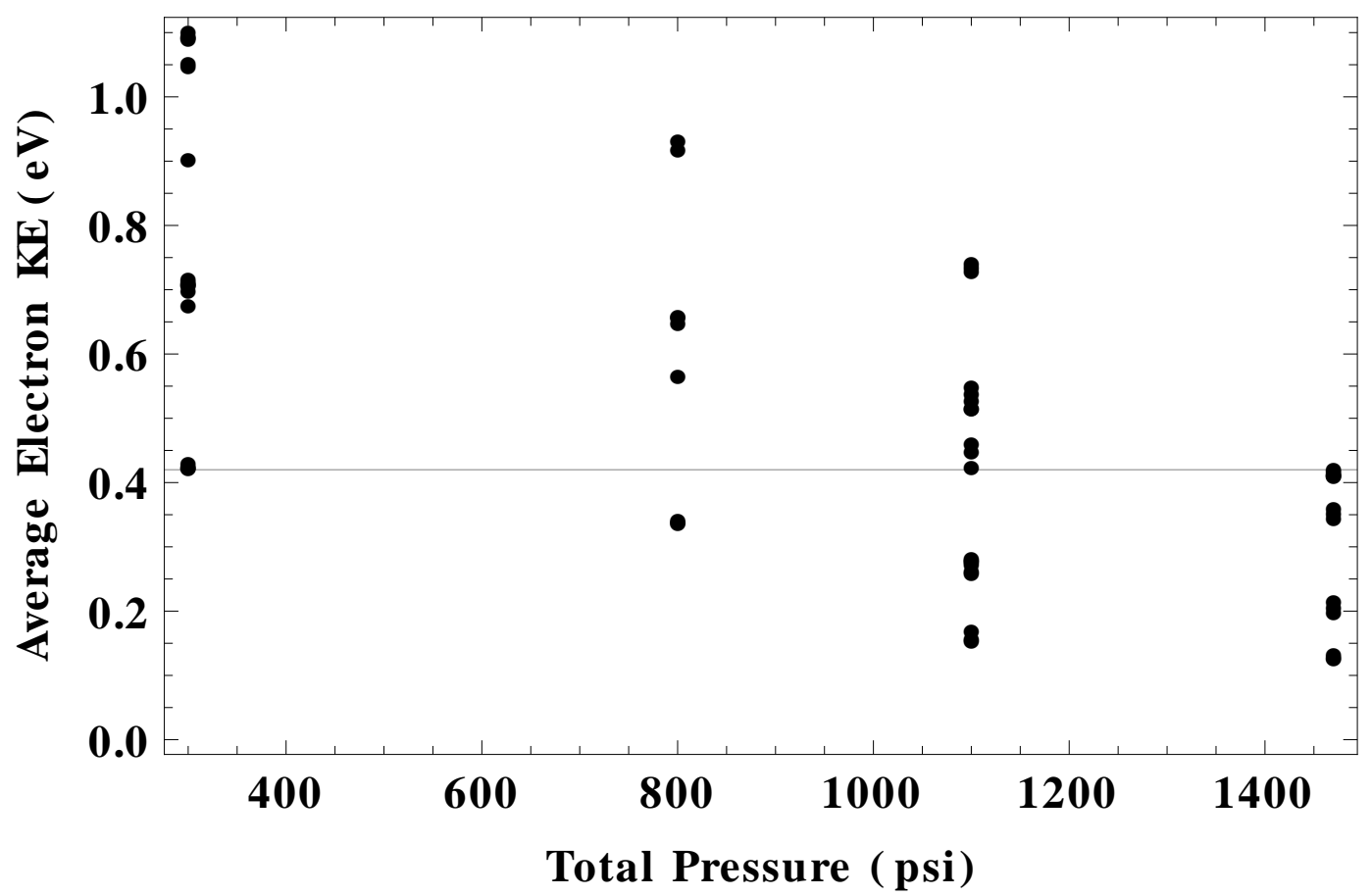

(a). The horizontal line is at $0.415 \mathrm{eV}$.

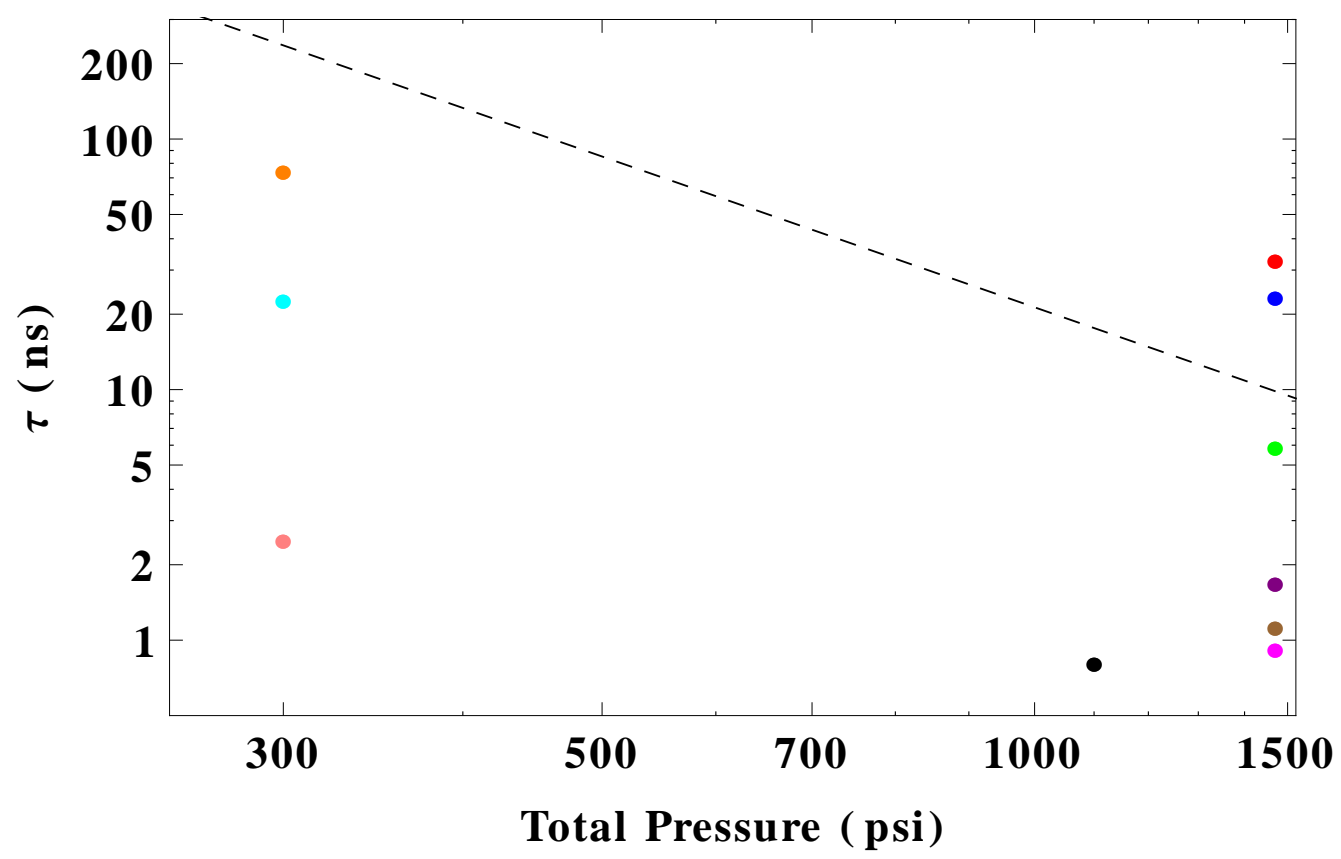

(b). Colors correspond to DA concentrations: $0.001 \%$ (red), $0.002 \%$ (blue), $0.01 \%$ (green), $0.025 \%$ (purple), $0.04 \%$ (orange), $0.05 \%$ (brown), $0.2 \%$ (cyan), $1 \%$ (magenta), $5 \%$ (pink), and $6.78 \%$ (black).

Figure 3.43. Average electron kinetic energy vs. gas pressure and $\tau$ vs. gas pressure for $0.4<\epsilon<0.43 \mathrm{eV}$. 
It has been observed that the lifetime of an electron decreases with increasing gas pressure, and further decreases with increasing dry air concentration. As the attachment rate of nitrogen is small compared to that of hydrogen and oxygen, it is safe to ignore it. Further, the results indicate that for most oxygen concentrations in hydrogen gas above 800 psi, the lifetime is less than $1 \mathrm{~ns}$.

Table 3.8 shows the fits of $\tau$ vs. $P$.

Table 3.8. Fits of the form $\tau=c_{1} P^{-c_{2}}$ for DA doped hydrogen.

\begin{tabular}{|c|c|c|c|}
\hline $\mathrm{E}(\mathrm{MV} / \mathrm{m})$ & Concentration (\%) & $c_{1}$ & $c_{2}$ \\
\hline 5 & 1 & $9.032 \times 10^{-5}$ & 1.653 \\
\hline 5 & 5 & $3.675 \times 10^{-6}$ & 1.278 \\
\hline 10 & 0.04 & 2.951 & 2.969 \\
\hline 10 & 0.2 & 0.171 & 2.610 \\
\hline 10 & 1 & $4.246 \times 10^{-3}$ & 2.182 \\
\hline 10 & 5 & $3.985 \times 10^{-5}$ & 1.574 \\
\hline 20 & 0.2 & 32.970 & 3.367 \\
\hline 20 & 1 & $8.302 \times 10^{-3}$ & 2.204 \\
\hline 20 & 5 & $4.835 \times 10^{-5}$ & 1.547 \\
\hline 25 & 0.2 & $6.240 \times 10^{5}$ & 4.746 \\
\hline 25 & 1 & 6.720 & 3.170 \\
\hline 40 & 0.04 & 28.49 & 3.110 \\
\hline 40 & 1 & $7.455 \times 10^{3}$ & 4.193 \\
\hline
\end{tabular}


3.3.1 Attachment Uncertainties. The error in the measurements gets larger with increasing pressure and dry air concentration, i.e., smaller attachment times. When the power absorbed by the plasma is small, the signal to noise ratio becomes smaller, making the fit to the front end of the power curve less accurate. Additionally, for small values of $\tau$ the time between data points (10 ns/point) becomes an issue, although the noise in the power curves contributes more. However both of these are insignificant compared to the limit of the time constant based on the precision of the algorithm used to calculate $\tau$. For this reason, much of the data reported at 1100 and 1470 psi is an upper limit. Unfortunately the entire data set is small, and so these points have been included in the fits of the results so as to increase the statistics and gain a better idea of the trends in the data. Table 3.9 summarizes the errors on $\tau$.

Table 3.9. Errors on $\tau$.

\begin{tabular}{lll}
\hline \hline Source & Affects & Estimate (\%) \\
\hline Gas pressure & Front end power & 10 \\
DA concentration & Front end power & 10 \\
Noise in power curve & Fitting & 10 \\
Recursion formula & $\tau$ & $50(?)$ \\
\hline
\end{tabular}

\subsection{Ion-Ion Recombination}

The method used to find ion-ion recombination rate is almost identical to that used in finding the electron attachment time. The recursion formula, Eq. 3.28 was used with functional form inputs for $\eta$ and $\tau$ as described in Sec. 3.3. The number of each particle vs. time, along with the respective energy losses, was used to calculate the power lost to the gas, which was fit to the measurement (see Fig. 3.35). The volume occupied by ions is assumed to be the same as in Sec. 3.2. The plots below 
show the value of $\eta$ corresponding to the flat top voltage in the cavity. Figures 3.44 and 3.45 show $\eta$ vs. DA concentration and oxygen number density for various pressures. 


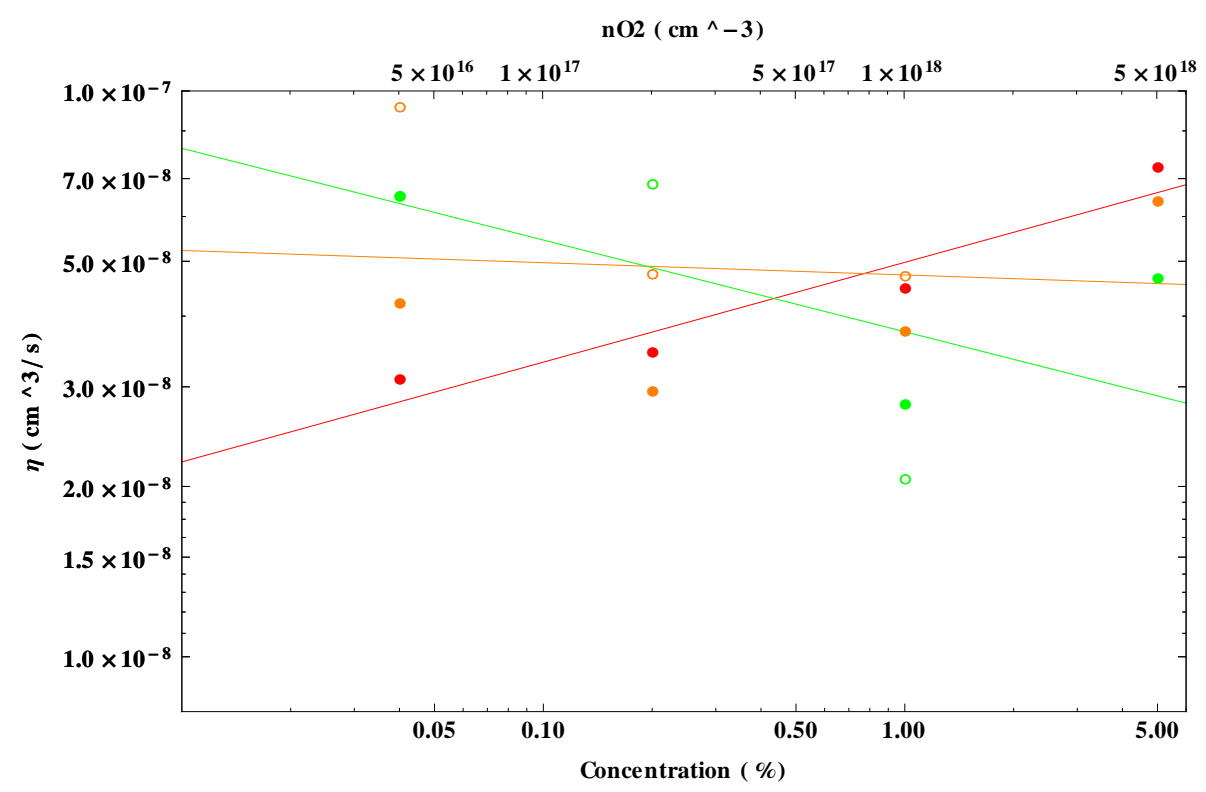

(a). $300 \mathrm{psi} ; 5 \mathrm{MV} / \mathrm{m}$ (red), $10 \mathrm{MV} / \mathrm{m}$ (orange), $18 \mathrm{MV} / \mathrm{m}$ (green).

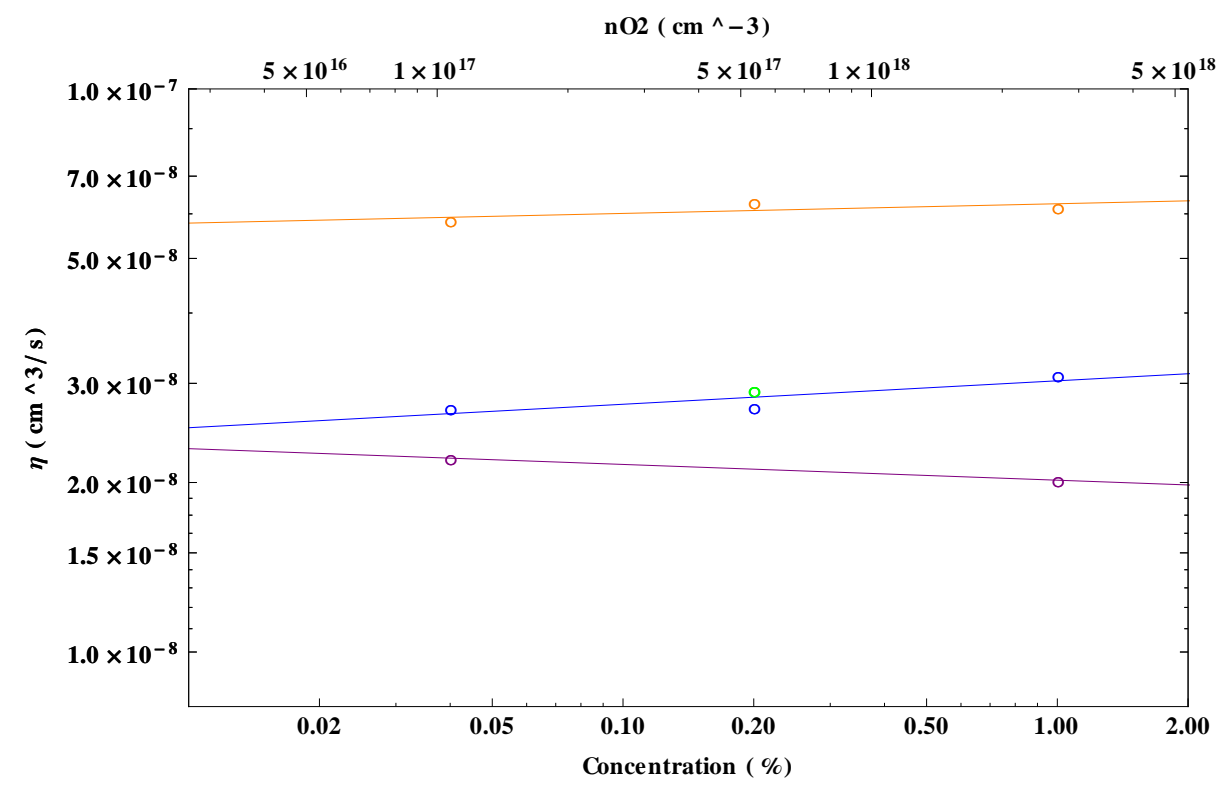

(b). $800 \mathrm{psi} ; 5 \mathrm{MV} / \mathrm{m}$ (red), $10 \mathrm{MV} / \mathrm{m}$ (orange), $20 \mathrm{MV} / \mathrm{m}$ (green), $25 \mathrm{MV} / \mathrm{m}$ (blue).

Figure 3.44. Ion-ion recombination rate vs. DA concentration and $\mathrm{O}_{2}$ density. The filled points are high beam intensity and the empty points are low beam intensity. The solid colored lines are fits to the data. 


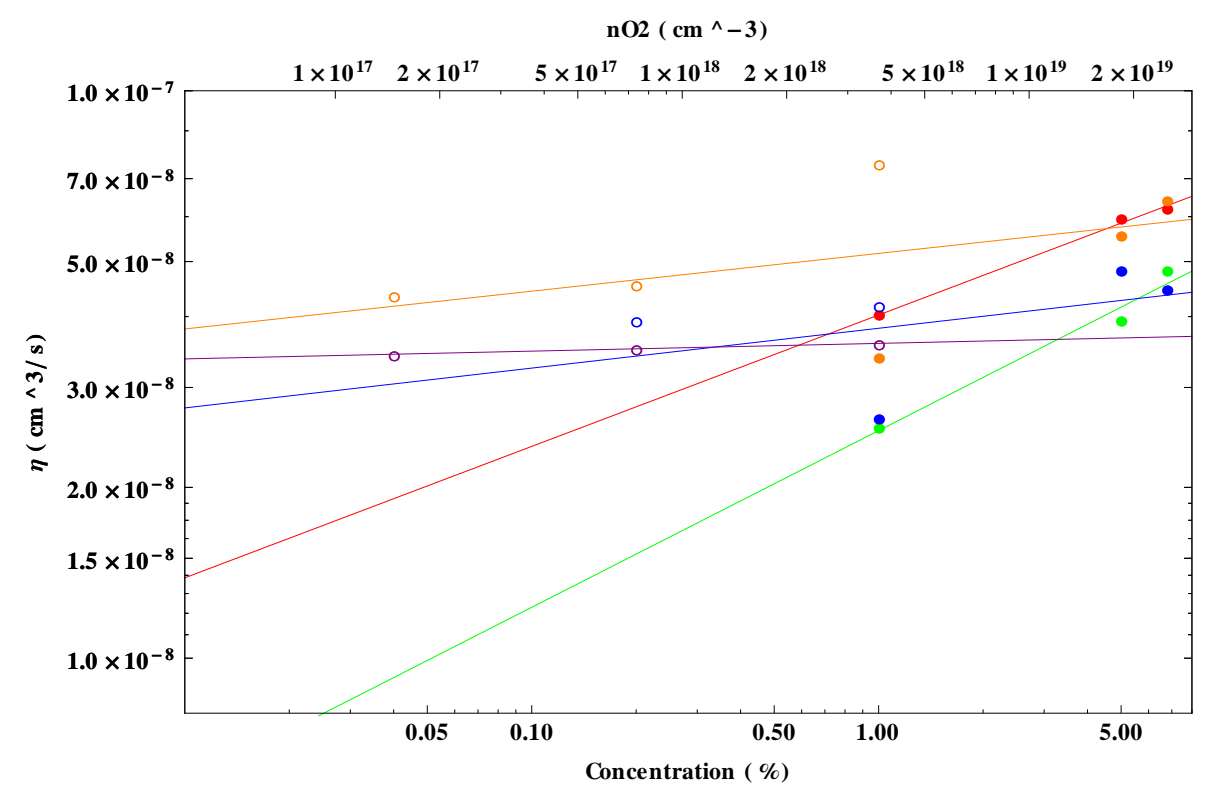

(a). $1100 \mathrm{psi} ; 5 \mathrm{MV} / \mathrm{m}$ (red), $10 \mathrm{MV} / \mathrm{m}$ (orange), $20 \mathrm{MV} / \mathrm{m}$ (green), $25 \mathrm{MV} / \mathrm{m}$ (blue).

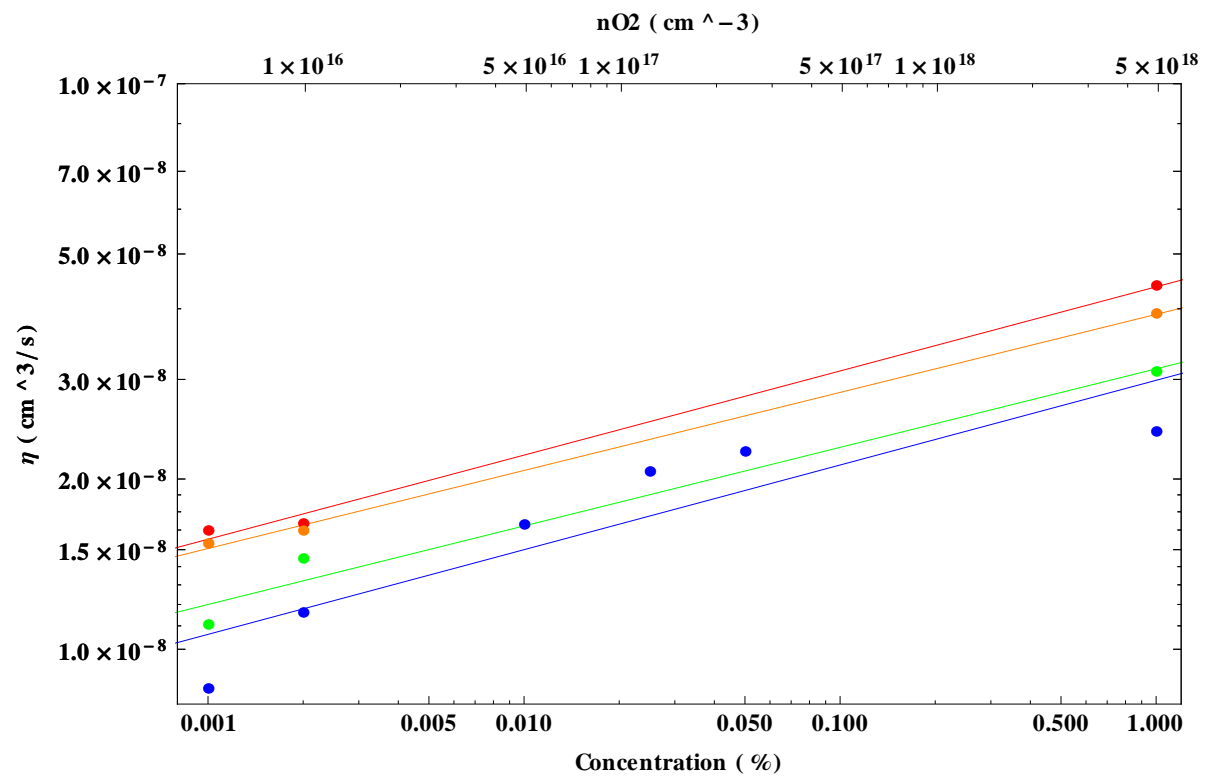

(b). $1470 \mathrm{psi} ; 5 \mathrm{MV} / \mathrm{m}$ (red), $10 \mathrm{MV} / \mathrm{m}$ (orange), $20 \mathrm{MV} / \mathrm{m}$ (green), $25 \mathrm{MV} / \mathrm{m}$ (blue).

Figure 3.45. Ion-ion recombination rate vs. DA concentration and $\mathrm{O}_{2}$ density. The filled points are high beam intensity and the empty points are low beam intensity. The solid colored lines are fits to the data. 
For the majority of the data, as the concentration of DA increases, the $\eta$ increases, if only slightly. The exception is at $300 \mathrm{psi}$, for which the energy loss is dominated by electrons, and so the ion contribution is not as clear. One would expect that as the oxygen (and hence $\mathrm{O}_{2}^{-}$) density increases, the ion-ion recombination rate will increase. $\mathrm{O}_{2}^{-}$are not as fast to recombine with hydrogen as electrons (there is approximately an order of magnitude difference in their respective recombination rates) due to its significantly smaller mobility.

Table 3.10 shows the fits for $\eta$ vs. DA concentration. 
Table 3.10. Fits of $\eta$ vs. concentration for DA doped hydrogen of the form $\eta=c_{1} C^{c_{2}}$.

\begin{tabular}{|c|c|c|c|}
\hline Pressure (psi) & $\mathrm{E}(\mathrm{MV} / \mathrm{m})$ & $c_{1}\left(10^{-8}\right)$ & $c_{2}$ \\
\hline 300 & 5 & 4.979 & 0.176 \\
\hline 300 & 10 & 4.729 & -0.022 \\
\hline 300 & 18 & 3.754 & -0.162 \\
\hline 800 & 10 & 6.253 & 0.017 \\
\hline 800 & 25 & 3.031 & 0.042 \\
\hline 800 & 40 & 2.019 & -0.028 \\
\hline 1100 & 5 & 4.026 & 0.232 \\
\hline 1100 & 10 & 5.168 & 0.067 \\
\hline 1100 & 20 & 2.518 & 0.311 \\
\hline 1100 & 25 & 3.814 & 0.070 \\
\hline 1100 & 40 & 3.588 & 0.014 \\
\hline 1470 & 5 & 4.374 & 0.149 \\
\hline 1470 & 10 & 3.910 & 0.138 \\
\hline 1470 & 20 & 3.132 & 0.139 \\
\hline 1470 & 25 & 2.989 & 0.150 \\
\hline
\end{tabular}


Figures 3.46 and 3.47 show $\eta$ vs. $X$ for various pressures. The colors correspond to DA concentrations and the lines are fits to the data. 


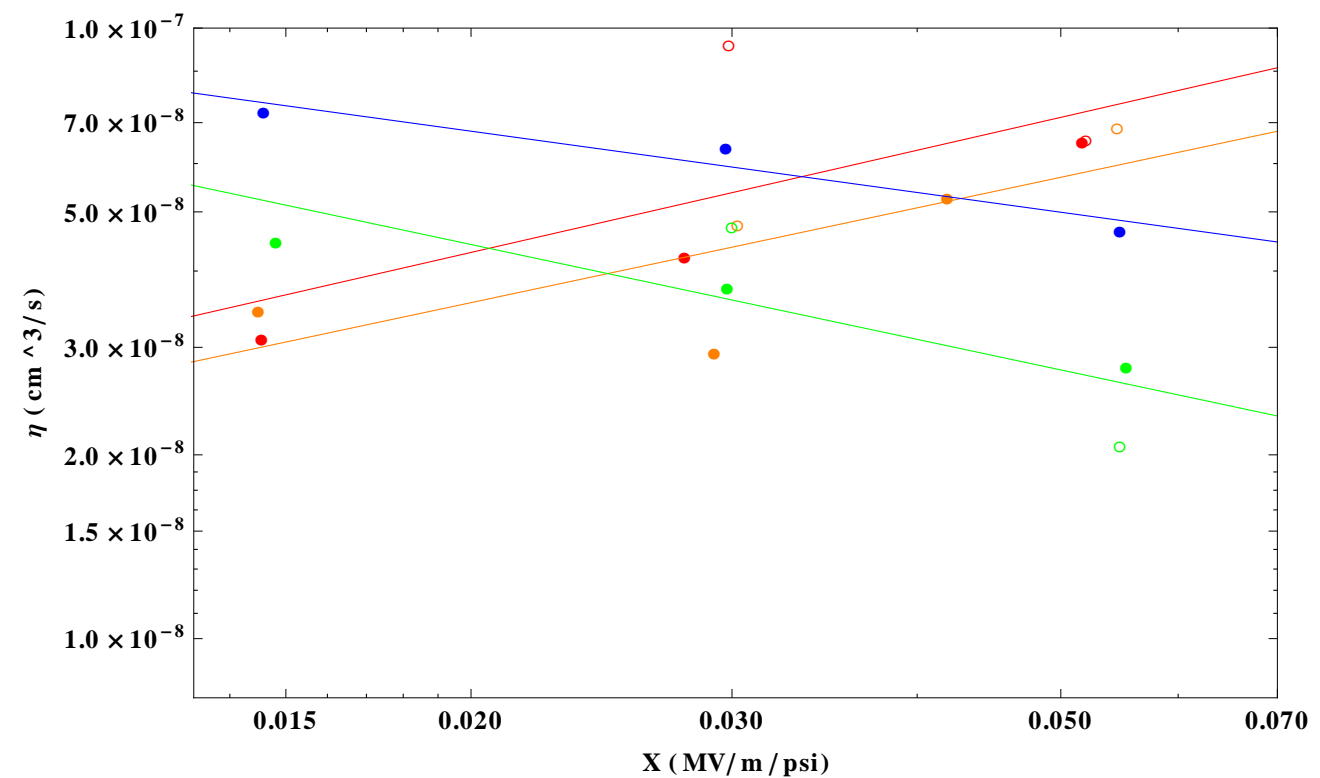

(a). $300 \mathrm{psi} ; 0.04 \%$ (red), $0.2 \%$ (orange), $1 \%$ (green), and $5 \%$ (blue).

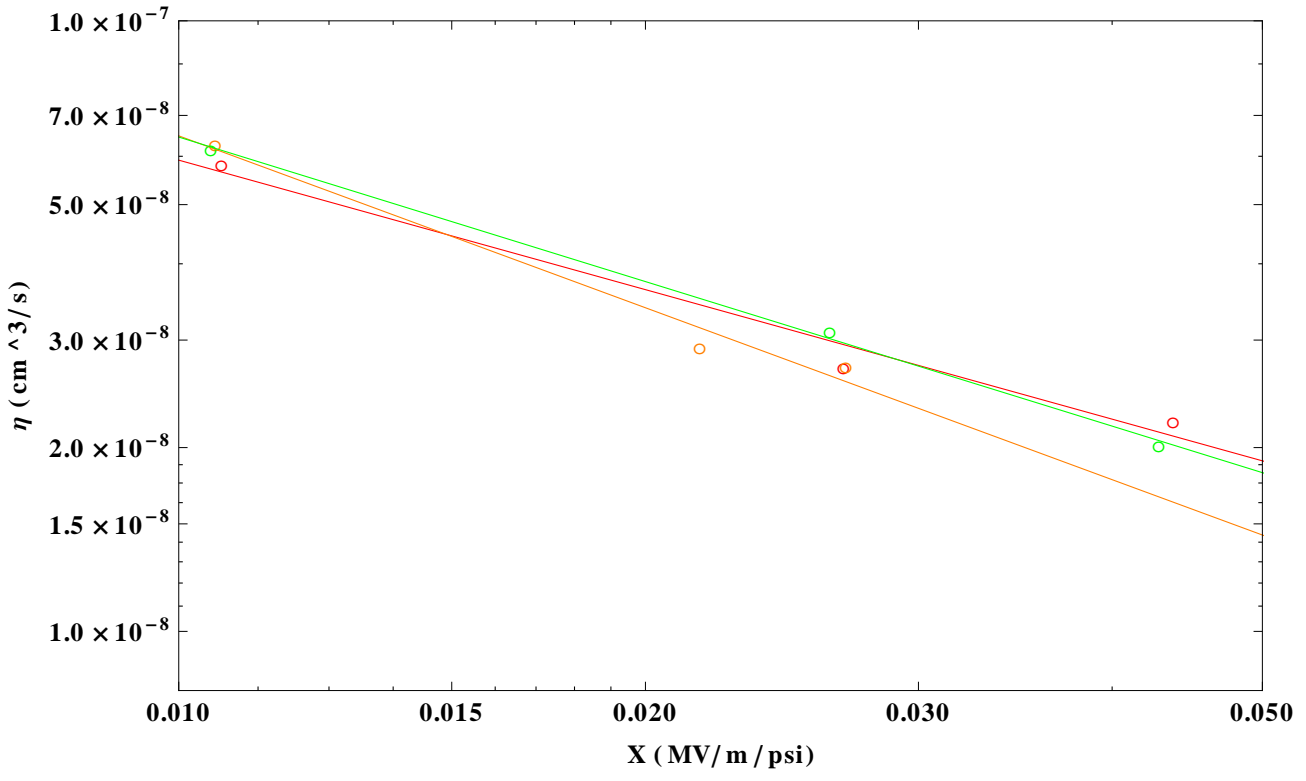

(b). $800 \mathrm{psi} ; 0.04 \%$ (red), $0.2 \%$ (orange), and $1 \%$ (green).

Figure 3.46. Ion-ion recombination rate vs. $X$. The filled points are high beam intensity and the empty points are low beam intensity. The solid lines are fits to the data. 


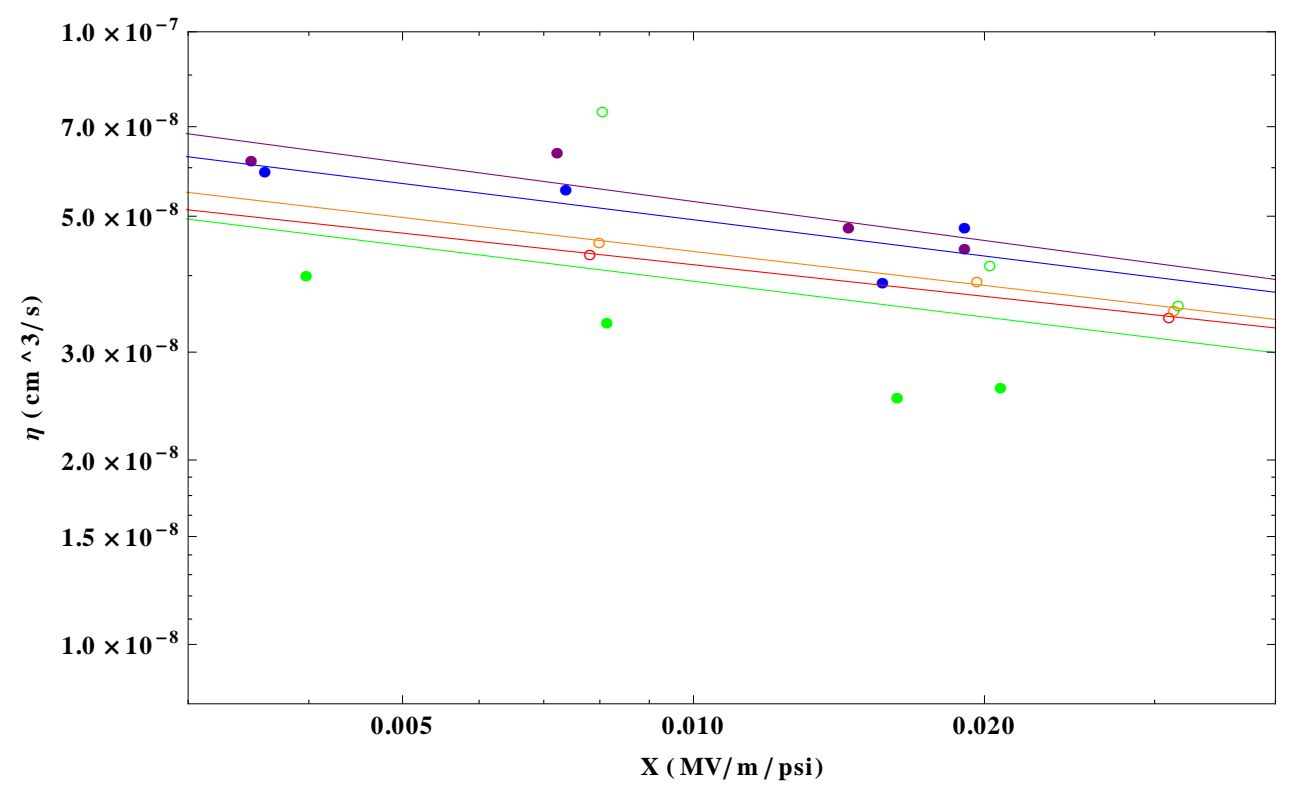

(a). $1100 \mathrm{psi} ; 0.04 \%$ (red), $0.2 \%$ (orange), $1 \%$ (green), $5 \%$ (blue), and $6.78 \%$ (purple).

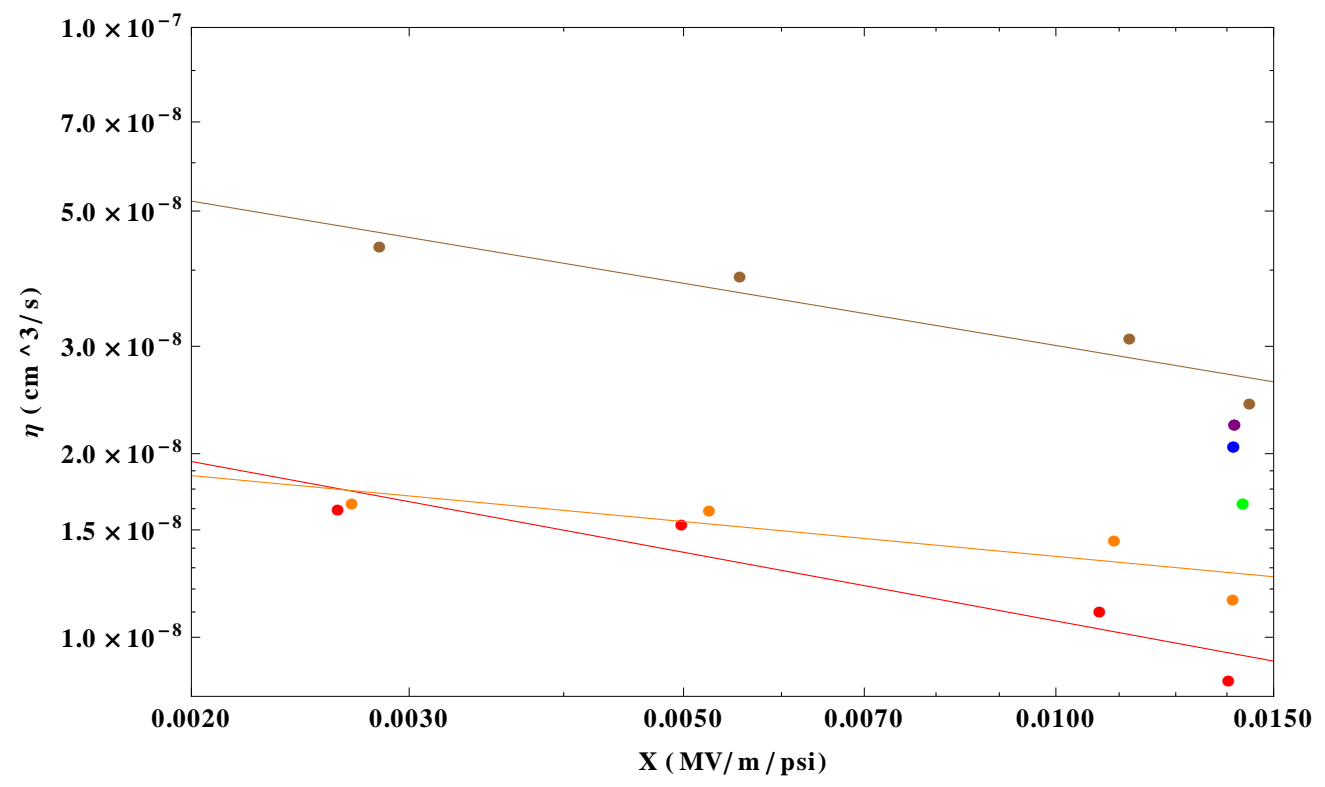

(b). 1470 psi; $0.001 \%$ (red), $0.002 \%$ (orange), $0.01 \%$ (green), $0.025 \%$ (blue), $0.05 \%$ (purple), and $1 \%$ (brown).

Figure 3.47. Ion-ion recombination rate vs. $X$. The filled points are high beam intensity and the empty points are low beam intensity. The solid lines are fits to the data. 
As described in Sec. 1.2.7, for low gas densities, ions must gain enough energy to find other ions while losing energy through collisions with neutral molecules. This can be seen clearly in Fig. 3.46a at 300 psi and 0.04 and $0.2 \%$ DA. In this region, the density of negative ions is small, so increasing $X$ increases the ion energy, hence the recombination rate becomes larger. It appears as if a transition occurs between 0.2 and $1 \%$ DA at 300 psi. For higher concentrations and subsequently higher pressures, there are sufficient negative ions so that the positive-negative ion collision rate is not an issue. Here we see the ion-ion recombination rate follow that of the electronhydrogen recombination rate in that the cross section for interaction must fall off with increasing $X$. Additionally, if the recombination rate of $\mathrm{O}_{2}^{-}$with $\mathrm{O}_{2}^{+}$from Tab. 1.6 is considered, increasing gas temperature decreases the recombination rate.

Table 3.11 shows the fits of $\eta$ vs. $X$. 
Table 3.11. Fits of $\eta$ vs. $X$ for DA doped hydrogen of the form $\eta=c_{1} X^{c_{2}}$.

\begin{tabular}{|c|c|c|c|}
\hline Pressure (psi) & Concentration (\%) & $c_{1}\left(10^{-9}\right)$ & $c_{2}$ \\
\hline 300 & 0.04 & 377.6 & 0.5556 \\
\hline 300 & 0.2 & 267.2 & 0.516 \\
\hline 300 & 1 & 5.870 & -0.516 \\
\hline 300 & 5 & 18.38 & -0.334 \\
\hline 800 & 0.04 & 2.305 & -0.705 \\
\hline 800 & 0.2 & 0.873 & -0.935 \\
\hline 800 & 1 & 1.726 & -0.786 \\
\hline 1100 & 0.04 & 18.93 & -0.171 \\
\hline 1100 & 0.2 & 18.74 & -0.184 \\
\hline 1100 & 1 & 16.03 & -0.194 \\
\hline 1100 & 5 & 19.95 & -0.197 \\
\hline 1100 & 6.78 & 19.97 & -0.211 \\
\hline 1470 & 0.001 & 1.899 & -0.374 \\
\hline 1470 & 0.002 & 5.668 & -0.190 \\
\hline 1470 & 1 & 6.325 & -0.339 \\
\hline
\end{tabular}




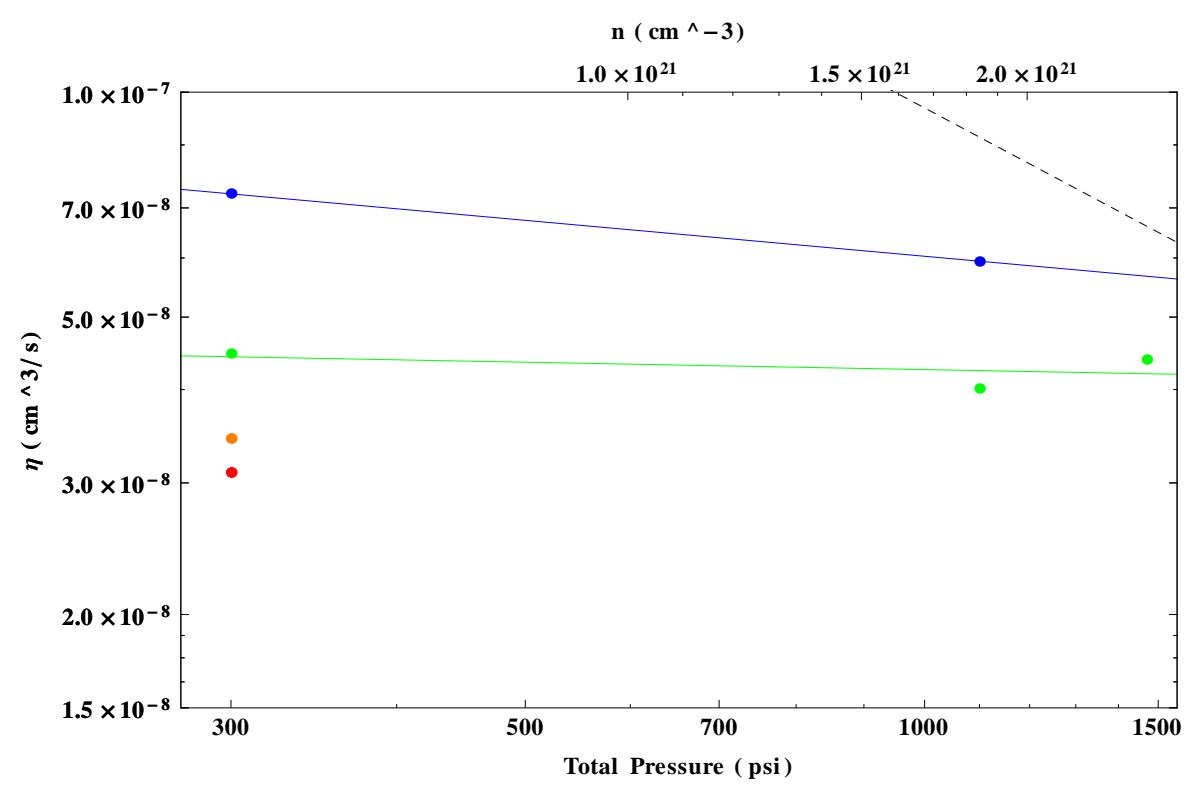

(a). $5 \mathrm{MV} / \mathrm{m} ; 0.04 \%$ (red), $0.2 \%$ (orange), $1 \%$ (green), and $5 \%$ (blue).

Figure 3.48. Ion-ion recombination rate vs. total pressure and number density. The filled points are high beam intensity and the empty points are low beam intensity. The solid lines are fits to the data. The black dashed line is an extrapolation from Fig. 1.31.

Figures 3.48, 3.49, and 3.50 show $\eta$ vs. pressure and number density for various electric fields. The colors correspond to concentrations and the solid lines are fits to the data. The black dashed line is an extrapolation to the pressure range measured here of the ion-ion recombination rate based on the general rate coefficient given in Fig. 1.31. 


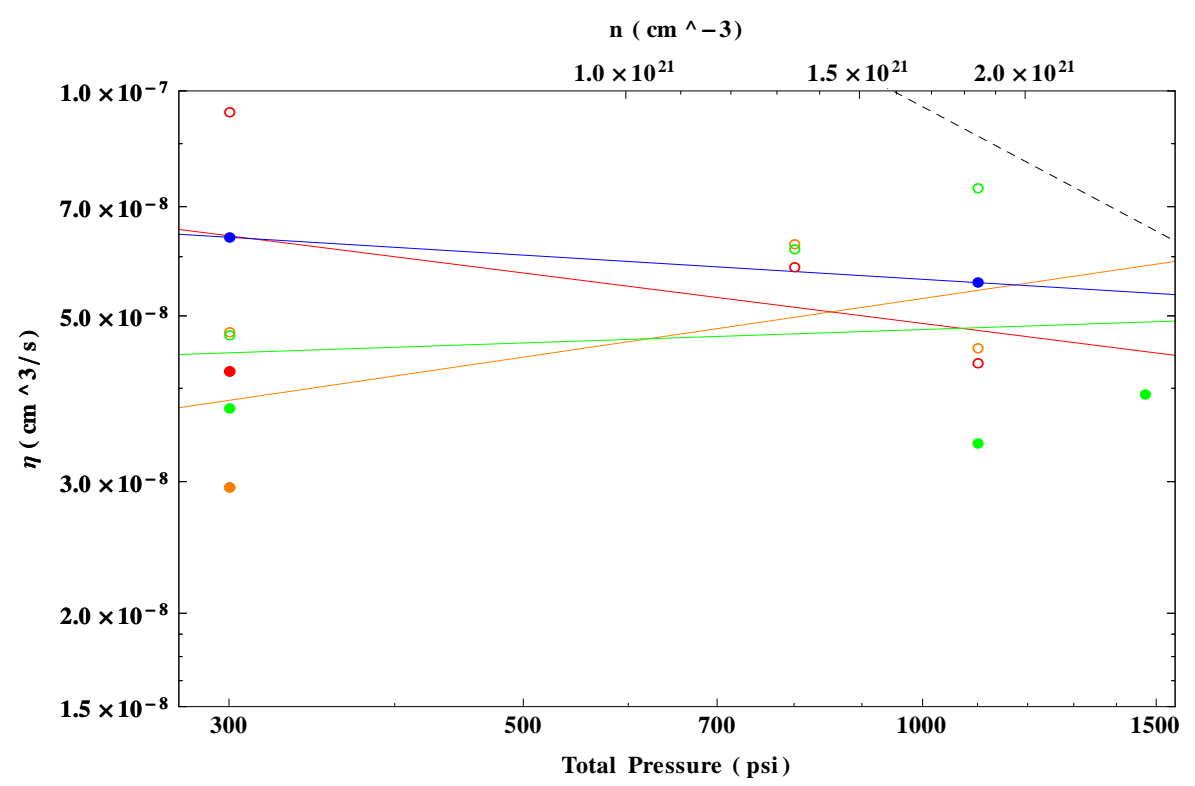

(a). $10 \mathrm{MV} / \mathrm{m} ; 0.04 \%$ (red), $0.2 \%$ (orange), $1 \%$ (green), and $5 \%$ (blue).

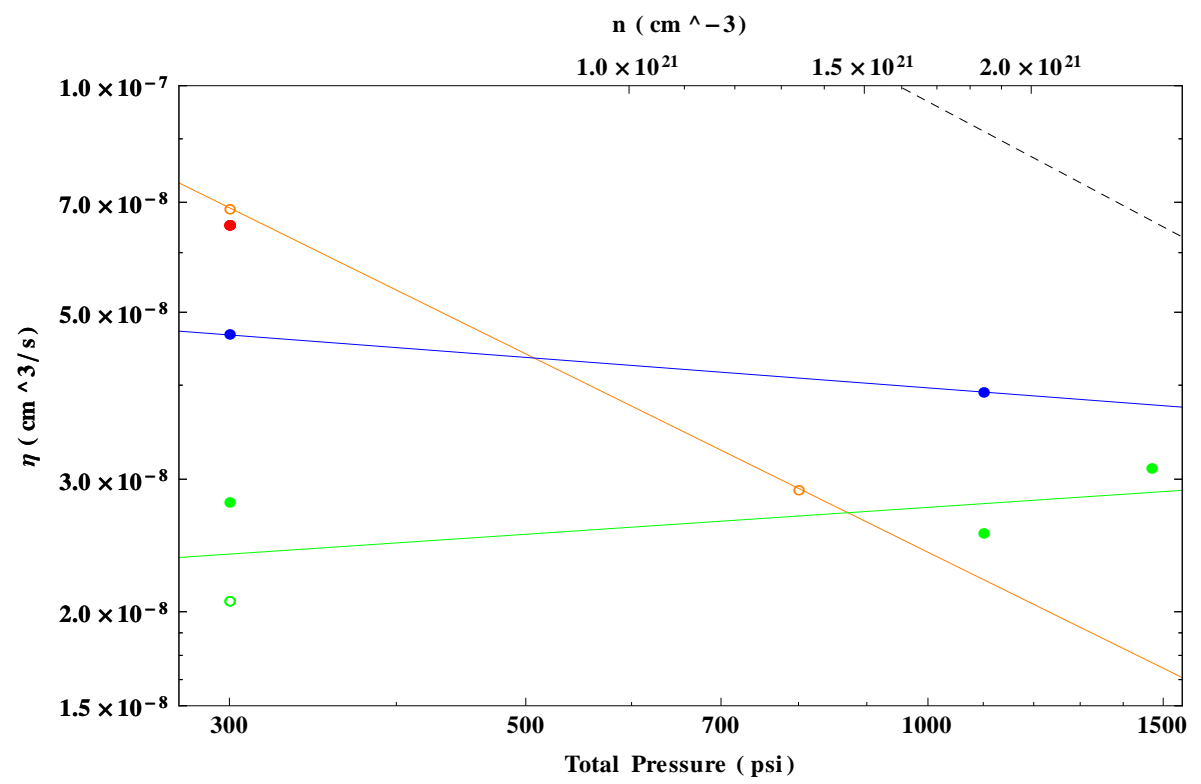

(b). $20 \mathrm{MV} / \mathrm{m} ; 0.04 \%$ (red), $0.2 \%$ (orange), $1 \%$ (green), and $5 \%$ (blue).

Figure 3.49. Ion-ion recombination rate vs. total pressure and number density. The filled points are high beam intensity and the empty points are low beam intensity. The solid lines are fits to the data. The black dashed line is an extrapolation from Fig. 1.31. 


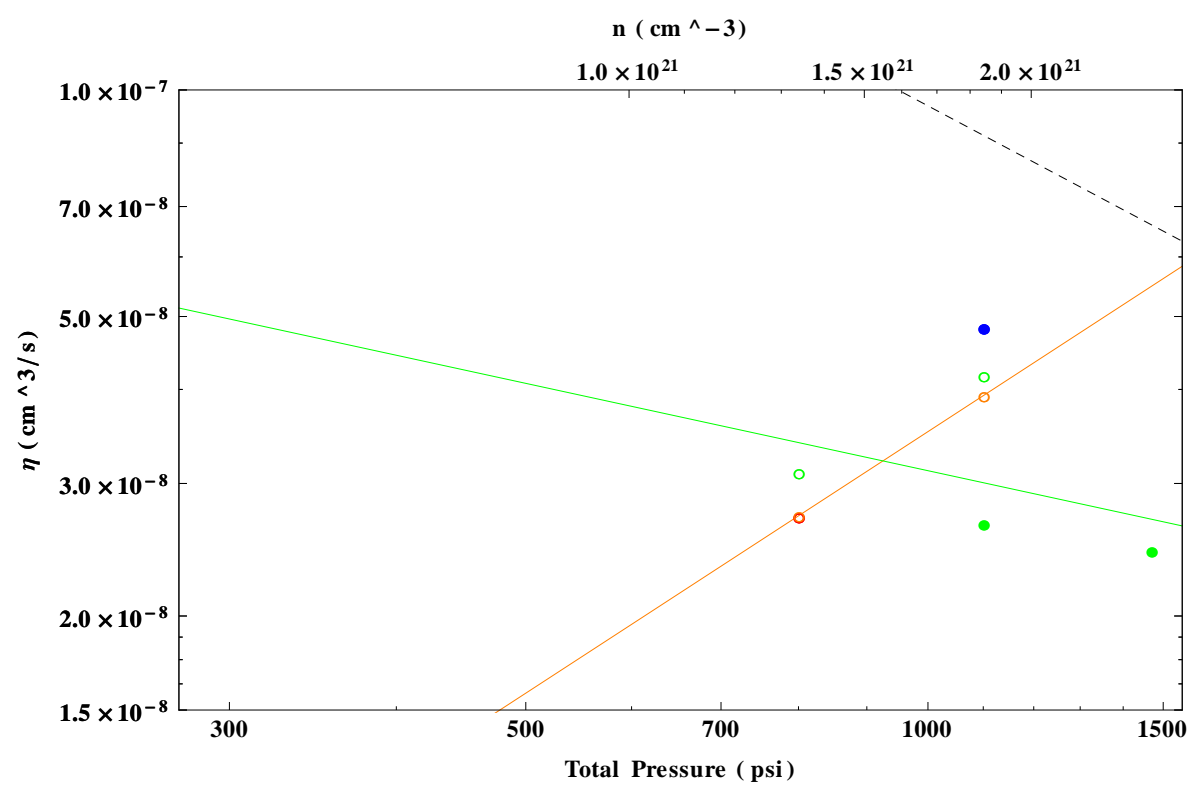

(a). $25 \mathrm{MV} / \mathrm{m} ; 0.04 \%$ (red), $0.2 \%$ (orange), $1 \%$ (green), and $5 \%$ (blue).

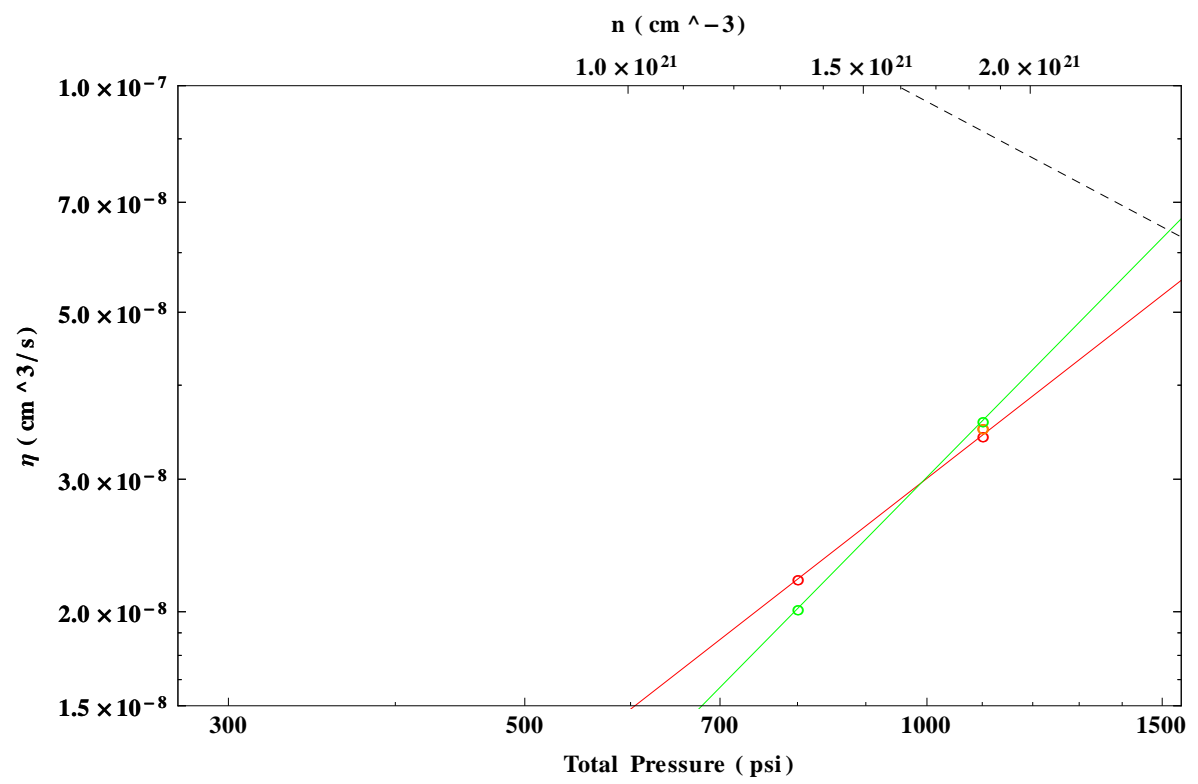

(b). $40 \mathrm{MV} / \mathrm{m} ; 0.04 \%$ (red), $0.2 \%$ (orange), $1 \%$ (green), and $5 \%$ (blue).

Figure 3.50. Ion-ion recombination rate vs. total pressure and number density. The filled points are high beam intensity and the empty points are low beam intensity. The solid lines are fits to the data. The black dashed line is an extrapolation from Fig. 1.31. 
With the exception of $40 \mathrm{MV} / \mathrm{m}$, most of the data shows only a slight change in $\eta$ with respect to pressure. It would appear that for the density range reported here, the ion-ion recombination rate does not greatly depend on pressure. The entire range of $\eta$ measurements is only roughly an order of magnitude. If one considers the data in Tab. 1.6, $\mathrm{O}_{2}^{-}$has been previously measured to recombine with diatomic molecules $\left(\mathrm{O}_{2}^{+}\right.$and $\left.N_{2}^{+}\right)$at rates of $10^{-8}-10^{-7} \frac{\mathrm{cm}^{3}}{\mathrm{~s}}$, which is in good agreement with our data.

Table 3.12 shows the fits of $\tau$ vs. $P$. 
Table 3.12. Fits of $\eta$ vs. $P$ for DA doped hydrogen of the form $\eta=c_{1} P^{c_{2}}$.

\begin{tabular}{|c|c|c|c|}
\hline $\mathrm{E}(\mathrm{MV} / \mathrm{m})$ & Concentration (\%) & $c_{1}$ & $c_{2}$ \\
\hline 5 & 1 & $5.337 \times 10^{-8}$ & -0.033 \\
\hline 5 & 5 & $1.817 \times 10^{-7}$ & -0.160 \\
\hline 10 & 0.04 & $2.300 \times 10^{-7}$ & -0.224 \\
\hline 10 & 0.2 & $8.712 \times 10^{-9}$ & 0.261 \\
\hline 10 & 1 & $3.177 \times 10^{-8}$ & 0.060 \\
\hline 10 & 5 & $1.177 \times 10^{-7}$ & -0.108 \\
\hline 20 & 0.2 & $1.014 \times 10^{-5}$ & -0.875 \\
\hline 20 & 1 & $1.211 \times 10^{-8}$ & 0.119 \\
\hline 20 & 5 & $1.004 \times 10^{-7}$ & -0.134 \\
\hline 25 & 0.2 & $1.210 \times 10^{-11}$ & 1.154 \\
\hline 25 & 1 & $4.473 \times 10^{-7}$ & -0.385 \\
\hline 40 & 0.04 & $2.143 \times 10^{-12}$ & 1.382 \\
\hline 40 & 1 & $1.172 \times 10^{-13}$ & 1.804 \\
\hline
\end{tabular}


3.4.1 Ion-ion Recombination Uncertainties. The errors associated with the ion-ion recombination measurement are similar to those for the attachment time measurement, as they were made using the same recursion formula. Here, for low pressures and concentrations, $\eta$ is fit near the end of the power curve due to electrons dominating the energy loss at the front. However, electrons still contribute at the end at $300 \mathrm{psi}$, so the error in the $\tau$ measurement is tied into the $\eta$ measurement at this pressure. For large pressures, the electrons become attached very quickly, and the front end power loss comes primarily from the ions. It can be seen in Fig. 3.35d that the calculated energy loss in the first $2 \mu$ s overshoots the measurement, which could not be rectified. The tail of the power curve at high pressure is also more noisy than at low pressure, even after averaging over $90 \mathrm{~ns}$.

The energy loss per ion used also presents a source of error. Constant mobilities for $\mathrm{H}_{5}^{+}$and $\mathrm{O}_{2}^{-}$were used to perform the calculation, however it is clear the mobility varies with gas pressure. This has a direct impact on the power loss calculation. The exact species of ions is also unknown. Based on the electron-hydrogen recombination measurements there is good reason to believe that $n_{\mathrm{H}_{5}^{+}} \gg n_{\mathrm{H}_{3}^{+}}$for most cases, however larger clusters of hydrogen are possible, whose mobililities are unknown. Oxygen could also have reacted to form oxygen clusters, or molecules containing oxygen and hydrogen or nitrogen. The potential for water formation is of course present, however only in very small amounts. Table 3.13 summarizes the errors on $\eta$.

Due to complexity of the system (hydrogen, oxygen, nitrogen) and the inability to determine specific molecular species in the cavity, an order of magnitude estimate for the ion-ion recombination rate is the best that can be accomplished at this point. 
Table 3.13. Errors on $\eta$.

\begin{tabular}{lll}
\hline \hline Source & Affects & Estimate (\%) \\
\hline Gas pressure & Front end power & 10 \\
DA concentration & Front end power & 10 \\
Noise in power curve & Fitting & 10 \\
$d w$ for ions & Ion power loss & 20 \\
Ion species & Ion power loss & 10 \\
\hline
\end{tabular}




\section{CHAPTER 4}

\section{CONCLUSION}

The energy loss per RF cycle for an electron-hydrogen ion pair in a plasma within a high pressure gas filled radio frequency cavity has been measured at $10^{-18}-$ $10^{-16} \mathrm{~J}(6-600 \mathrm{eV})$, and for an oxygen ion-hydrogen ion pair at $10^{-20}-10^{-18} \mathrm{~J}$ $(0.06-6 \mathrm{eV})$. A saturation of the energy loss at high pressure has been observed. The recombination rate of electrons in hydrogen is on the order of $10^{-6} \frac{\mathrm{cm}^{3}}{\mathrm{~s}}$, and indicates that $\mathrm{H}_{5}^{+}$is the dominant hydrogen ion in the HPRF cavity. The ion-ion recombination rate has been measured at $10^{-8}-10^{-7} \frac{\mathrm{cm}^{3}}{\mathrm{~s}}$. The lifetime of an electron in dry air-doped hydrogen at pressures above $800 \mathrm{psi}$ and oxygen concentrations above $0.2 \%$ has an upper limit of $\approx 1 \mathrm{~ns}$, while high pressure, high concentration lifetimes appear to be well below that.

These results may be applied to help validate the use of an HPRF cavity in the cooling-channel of a Neutrino Factory or Muon Collider.

\subsection{Neutrino Factory}

The cooling requirements are less strict and number of muons per bunch smaller for a Neutrino Factory than for a Muon Collider (see Tables 1.2 and 1.3). Table 1.1 lists the RF requirements for a Neutrino Factory. We will assume an HPRF cavity will be used, operated at: $201 \mathrm{MHz}, 15 \frac{\mathrm{MV}}{\mathrm{m}}$, with $100 \mathrm{~atm}$ (1470 psi) of $0.2 \%$ oxygen doped hydrogen at $293 \mathrm{~K}$. Each incident muon will produce roughly:

$$
N_{\text {pairs }}=\frac{\frac{d E}{d x} \rho L}{W_{i}}=\frac{4.2 \frac{\mathrm{MeV} \mathrm{cm}^{2}}{\mathrm{~g}} \times 2 \frac{\mathrm{g}}{\mathrm{mol}} \times \frac{1}{240.4} \frac{\mathrm{mol}}{\mathrm{cm}^{3}} \times 50 \mathrm{~cm}}{35.3 \mathrm{eV}}=48,300
$$

The measured energy loss per RF cycle per electron-ion pair at $100 \mathrm{~atm}$ of $1 \%$ dry air $(0.2 \%$ oxygen $)$ doped hydrogen is $3.1 \times 10^{-19} \frac{\mathrm{J}}{\text { cyclepair }}\left(1.93 \frac{\mathrm{eV}}{\text { cyclepair }}\right)$. This corresponds to $1.5 \times 10^{-14} \mathrm{~J}(93.6 \mathrm{keV})$ every RF cycle. If the momentum of incident muons (and hence $\frac{\mathrm{dE}}{\mathrm{dx}}$ ) does not change greatly, then the energy loss every RF cycle 
as a function of gas pressure can be given as:

$$
W(P)=1.50 \times 10^{-16} \frac{\mathrm{J}}{\mathrm{atm}} \times P=934 \frac{\mathrm{eV}}{\mathrm{atm}} \times P
$$

Using Eq. 1.23 and assuming the beam arrives at a $45^{\circ}$ phase angle, then the conventional beam loading would be:

$$
\begin{aligned}
W & =\frac{1}{2} q V=\frac{1}{2} 1.6 \times 10^{-19} \mathrm{C} \times 1.5 \times 10^{7} \sin (\pi / 4) \frac{\mathrm{eV}}{\mathrm{m}} \times 0.5 \mathrm{~m} \\
& =4.24 \times 10^{-13} \mathrm{~J}=2.62 \mathrm{MeV}
\end{aligned}
$$

Therefore the conventional beam loading would be more than an order of magnitude greater than the plasma loading.

For a copper pillbox cavity with the above parameters, the stored energy is 137.5 J. The total energy loss due to plasma loading for $10^{11}$ muons would be $1.5 \mathrm{~mJ}$ per RF cycle. If one assumes none of the ions recombine for the entire beam pulse (250 ns, or 50 bunches), this is still only $75 \mathrm{~mJ}$ lost due to plasma loading, which corresponds to a roughly $2 \%$ decrease in accelerating gradient.

\subsection{Muon Collider}

More cooling is needed for a Muon Collider than a Neutrino Factory. In addition to the bunching, phase rotation, and 4D cooling that forms the front end of a Muon Collider and provides all the necessary cooling for a Neutrino Factory, 6D cooling must be performed to reach the final emittance goal (see Tab. 1.2). To meet the luminosity criteria, a larger bunch charge is needed. We will consider the helical cooling-channel (HCC) described in Sec.1.1.3. The HCC employs HPRF cavities operated at $805 \mathrm{MHz}$ and $20 \frac{\mathrm{MV}}{\mathrm{m}}$, filled with $180 \mathrm{~atm}$ (2646 psi) of $1 \%$ oxygen doped hydrogen. If we assume the kinetic energy of the muons is $130 \mathrm{MeV}$ [1] and the cavities are $25 \mathrm{~cm}$ long and operated at $293 \mathrm{~K}$, then each incident muon would produce 
roughly:

$$
N_{\text {pairs }}=\frac{\frac{d E}{d x} \rho L}{W_{i}}=\frac{5 \frac{\mathrm{MeV} \mathrm{cm}^{2}}{\mathrm{~g}} \times 2 \frac{\mathrm{g}}{\mathrm{mol}} \times \frac{1}{133.6} \frac{\mathrm{mol}}{\mathrm{cm}^{3}} \times 25 \mathrm{~cm}}{35.3 \mathrm{eV}}=43,500
$$

Unfortunately we have no measurements of the energy loss in a cavity at this pressure and concentration. What we can do is use the measurements we have made to extrapolate to the HCC parameters. To begin, we take the fit to the $\tau$ versus $P$ data at $20 \frac{\mathrm{MV}}{\mathrm{m}}$ and $5 \% \mathrm{DA}$ (1\% oxygen) shown in Fig. 3.41a. Extrapolating $\tau$ to $180 \mathrm{~atm}$ (2646 psi) this gives us a lifetime of $0.245 \mathrm{~ns}$ for an electron. For an initial number of 43,500 electron-hydrogen ion pairs, the number of electrons fall and the number of $\mathrm{O}_{2}^{-}$rise as shown in Fig. 4.1.

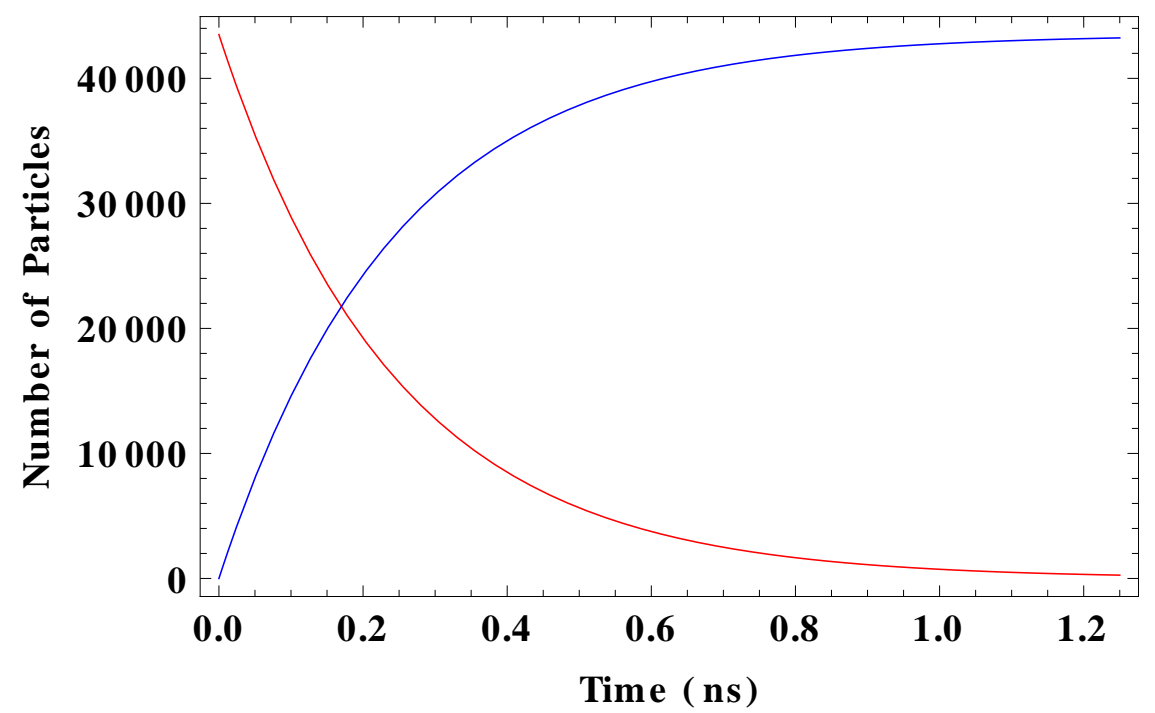

Figure 4.1. Number of electrons (red) and $\mathrm{O}_{2}^{-}$(blue) for one $\mathrm{RF}$ cycle with an initial using HCC parameters. The initial number of electron-hydrogen ion pairs is 43,500.

After one RF cycle (1.25 ns), only $0.6 \%$ of the initial electrons remain. As can be seen in Fig. 3.27, the energy loss per electron-hydrogen ion pair over one RF cycle appears to saturate at high pressure. For this reason, we will assume the $d w$ vs. $X$ curve for electrons and hydrogen ions follows that at 1520 psi (our highest pressure measurement) for pure hydrogen data, which can be found in Tab. 3.1. For ions, we will assume the $d w$ vs. $X$ curve follows that at 1470 psi with $1 \%$ DA for oxygen and 
hydrogen ions, which can be found in Tab. 3.2, using the same reasoning. Figure 4.2 shows $d w$ vs. time for one RF cycle.

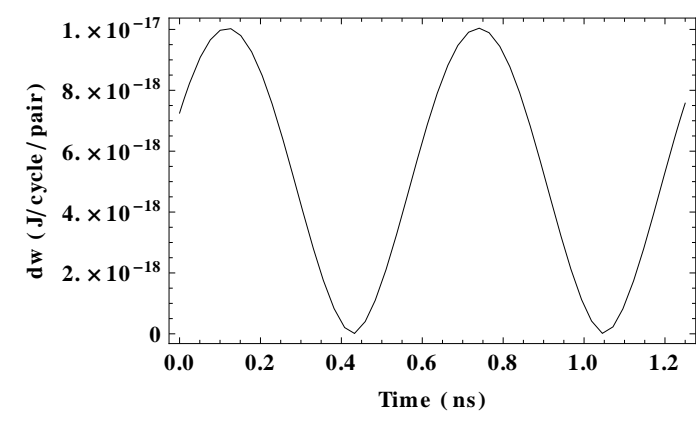

(a). Electron-hydrogen ion pairs.

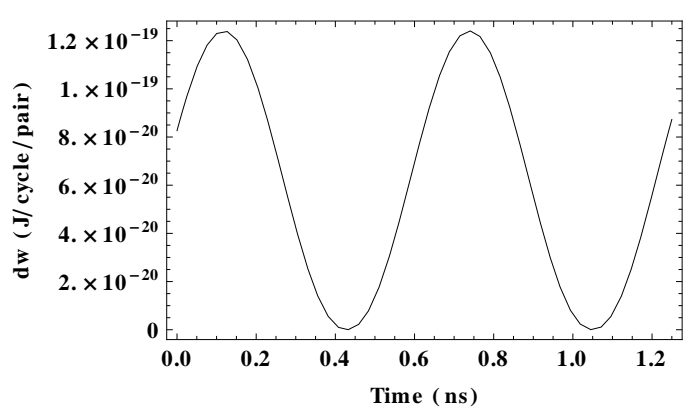

(b). Oxygen ion-hydrogen ion pairs.

Figure 4.2. $d w$ per particle pair over one RF cycle based on HCC parameters.

There are two orders of magnitude difference between the peaks of the two energy loss curves. If the number of particles vs. time is incorporated into $d w$ vs. time, the energy loss, $T(t)=d w(X(t)) \times N(t)$, vs. time is the result. This is shown in Fig. 4.3.

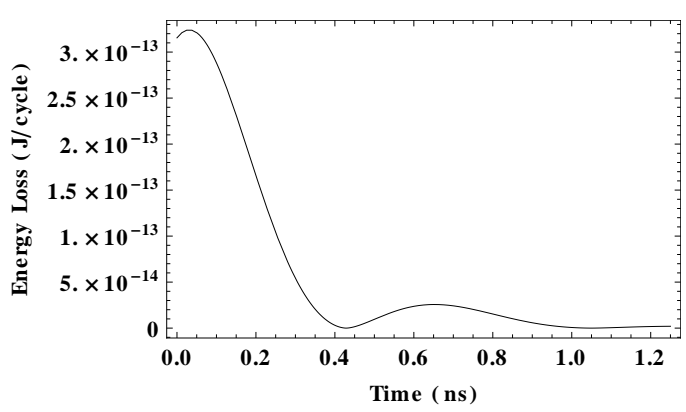

(a). Electron-hydrogen ion pairs.

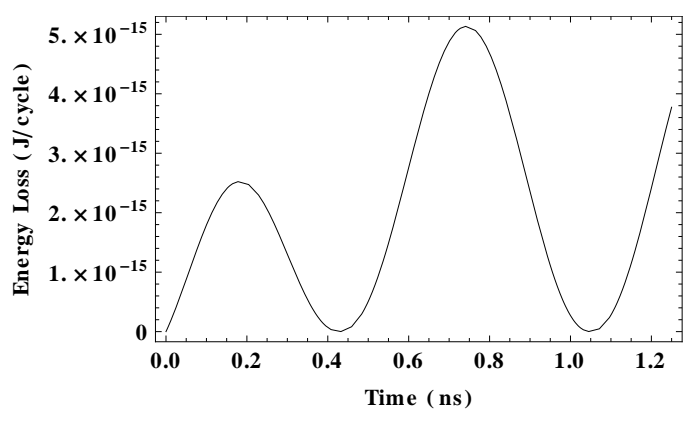

(b). Oxygen-hydrogen ion pairs.

Figure 4.3. Energy loss over one RF cycle based on HCC parameters.

The total energy loss is found by integrating over one RF period. For electrons and hydrogen ions, this is $3.91 \times 10^{-14} \mathrm{~J}(244 \mathrm{keV})$ per RF cycle, and for oxygen ionhydrogen ions this is $2.17 \times 10^{-15} \mathrm{~J}(13.5 \mathrm{keV})$ per RF cycle. Note that this method assumes the number of hydrogen ions is constant (i.e. there is no ion-ion recombi- 
nation) and that $n_{e}+n_{\mathrm{O}_{2}^{-}}=n_{\mathrm{H}_{5}^{+}}$. The hydrogen ion energy loss is incorporated in both Fig. $4.3 \mathrm{a}$ and $4.3 \mathrm{~b}$.

Conventional beam loading in this case is $2.83 \times 10^{-13} \mathrm{~J}(1.50 \mathrm{MeV})$ per muon, still nearly an order of magnitude larger. The total plasma loading for $10^{12}$ muons would then be $41.3 \mathrm{~mJ}$ per RF cycle. It is worth noting that this bunch intensity is three orders of magnitude larger than what was used the for the HPRF beam tests. A copper pillbox HPRF cavity used for a HCC would have $7.63 \mathrm{~J}$ stored energy. Assuming a constant rate of $41.3 \mathrm{~mJ}$ per bunch, the twelfth bunch would see $94 \%$ of the stored energy, or $97 \%$ of the accelerating voltage of the first bunch. This has the effect of heating the beam, or increasing the longitudinal phase space.

\subsection{Outlook}

The results of this experiment indicate that a high pressure gas filled radio frequency cavity would be a suitable means of preventing RF breakdown in multiTesla magnetic fields, while cooling a beam of muons at the same time. Another beam test using an HPRF would be beneficial in further cementing its validity for use in a Muon Collider cooling-channel. A pressure range that included 180 atm would provide measurements of the electron energy loss at the desired pressure. A spectroscopy or gas species sampling system would aid in identifying the species of molecules present in the plasma. Consistent measurements over a pressure range at a specific electron or ion energy would also be helpful. To ease analysis efforts, better care should be taken in the future to ensure the cavity conditions (gas pressure, electric field, etc) of data collected for pure hydrogen matches those collected with dopant gases. Finally, a higher resolution measurement of the lifetime of electrons in a dopant gas would significantly improve the quality of data. 
APPENDIX A

RUN CONDITIONS RUN I 
Table A.1. Run I run conditions.

\begin{tabular}{|c|c|c|c|c|c|c|c|c|c|c|}
\hline $\begin{array}{l}\text { Gas } \\
\text { Species }\end{array}$ & $\begin{array}{l}\text { Gas } \\
\text { Pres. } \\
(\mathrm{psi})\end{array}$ & $\begin{array}{l}\text { Beam } \\
\text { Int. }\end{array}$ & $\begin{array}{l}\text { Cavity } \\
\text { Grad. } \\
(\mathrm{MV} / \mathrm{m})\end{array}$ & Date & & Time & Long RF & Long Beam & $\begin{array}{l}\text { Additional } \\
\text { Date }\end{array}$ & $\begin{array}{l}\text { Additional } \\
\text { Time }\end{array}$ \\
\hline $\mathrm{N} 2$ & 500 & high & 10 & $12-\mathrm{Jul}$ & $\begin{array}{l}16: 34: 15- \\
16: 57: 08\end{array}$ & & $16: 59-17: 03$ & & & \\
\hline $\mathrm{N} 2$ & 500 & high & 20 & $12-\mathrm{Jul}$ & $\begin{array}{l}15: 40: 33- \\
16: 19: 53\end{array}$ & & & & & \\
\hline $\mathrm{N} 2$ & 500 & high & 30 & 12-Jul & $\begin{array}{l}16: 20: 53- \\
16: 33: 12\end{array}$ & & & & & \\
\hline $\mathrm{H} 2$ & 500 & low & 10 & & & & & & & \\
\hline $\mathrm{H} 2$ & 500 & low & 20 & & & & & & & \\
\hline $\mathrm{H} 2$ & 500 & low & 30 & & & & & & & \\
\hline $\mathrm{H} 2$ & 500 & med & 10 & 27-Jul & $\begin{array}{l}16: 35: 47- \\
16: 45: 02\end{array}$ & & & & & \\
\hline $\mathrm{H} 2$ & 500 & med & 20 & 27-Jul & $\begin{array}{l}16: 10: 57- \\
16: 21: 25\end{array}$ & $\begin{array}{l}16: 46: 03- \\
16: 48: 35\end{array}$ & & & & \\
\hline $\mathrm{H} 2$ & 500 & med & 25 & 27-Jul & $\begin{array}{l}16: 25: 31- \\
16: 34: 46\end{array}$ & & & & & \\
\hline $\mathrm{H} 2$ & 500 & med & 30 & 27-Jul & $\begin{array}{l}16: 22: 26- \\
16: 24: 30\end{array}$ & & & & & \\
\hline $\mathrm{H} 2$ & 500 & high & 10 & 22-Jul & $\begin{array}{l}17: 00: 47- \\
17: 16: 16\end{array}$ & & & & 27-Jul & $\begin{array}{l}13: 04: 17- \\
13: 15: 10\end{array}$ \\
\hline $\mathrm{H} 2$ & 500 & high & 20 & 22-Jul & $\begin{array}{l}16: 04: 49- \\
16: 42: 32\end{array}$ & & $\begin{array}{l}17: 31: 52- \\
17: 47: 08\end{array}$ & & 27-Jul & $\begin{array}{l}12: 18: 04- \\
12: 46: 50\end{array}$ \\
\hline $\mathrm{H} 2$ & 500 & high & 25 & 22-Jul & $\begin{array}{l}17: 17: 18- \\
17: 30: 50\end{array}$ & & & & 27-Jul & $\begin{array}{l}12: 52: 59- \\
13: 03: 15\end{array}$ \\
\hline $\mathrm{H} 2$ & 500 & high & 30 & $22-\mathrm{Jul}$ & $\begin{array}{l}16: 43: 47- \\
16: 59: 47\end{array}$ & & & & 27-Jul & $\begin{array}{l}12: 47: 51- \\
12: 51: 28\end{array}$ \\
\hline $\mathrm{H} 2$ & 800 & low & 10 & 25-Jul & $\begin{array}{l}16: 03: 46- \\
16: 15: 50\end{array}$ & & & & & \\
\hline $\mathrm{H} 2$ & 800 & low & 20 & 25-Jul & $\begin{array}{l}15: 06: 53- \\
15: 28: 39\end{array}$ & $\begin{array}{l}15: 44: 25- \\
15: 50: 43\end{array}$ & $\begin{array}{l}16: 18: 59- \\
16: 24: 15\end{array}$ & $\begin{array}{l}16: 25: 18- \\
16: 27: 24\end{array}$ & & \\
\hline $\mathrm{H} 2$ & 800 & low & 30 & 25-Jul & $\begin{array}{l}15: 51: 47- \\
16: 02: 43\end{array}$ & & & & & \\
\hline $\mathrm{H} 2$ & 800 & med & 10 & 27-Jul & $\begin{array}{l}14: 26: 25- \\
14: 38: 44\end{array}$ & & & & & \\
\hline $\mathrm{H} 2$ & 800 & med & 20 & 27-Jul & $\begin{array}{l}13: 49: 27- \\
14: 15: 07\end{array}$ & $\begin{array}{l}14: 39: 46- \\
14: 45: 04\end{array}$ & & & & \\
\hline $\mathrm{H} 2$ & 800 & med & 30 & 27-Jul & $\begin{array}{l}14: 16: 09- \\
14: 25: 23\end{array}$ & & & & & \\
\hline $\mathrm{H} 2$ & 800 & high & 10 & 14-Jul & $\begin{array}{l}13: 05: 08- \\
13: 16: 30\end{array}$ & & & & & \\
\hline $\mathrm{H} 2$ & 800 & high & 20 & 14-Jul & $\begin{array}{l}12: 07: 00- \\
12: 51: 22\end{array}$ & & $\begin{array}{l}13: 18: 34- \\
13: 20: 38\end{array}$ & & & \\
\hline
\end{tabular}




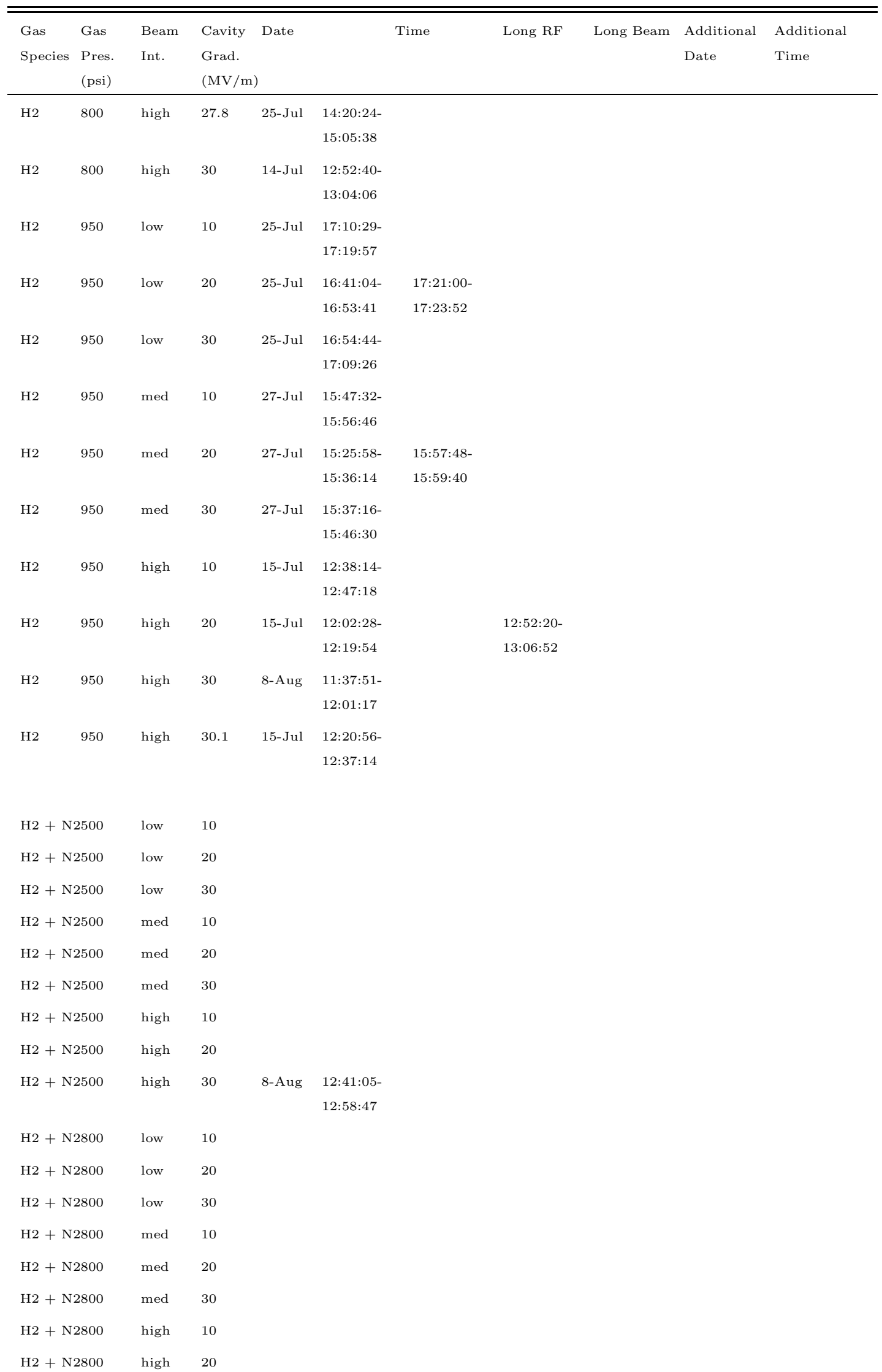




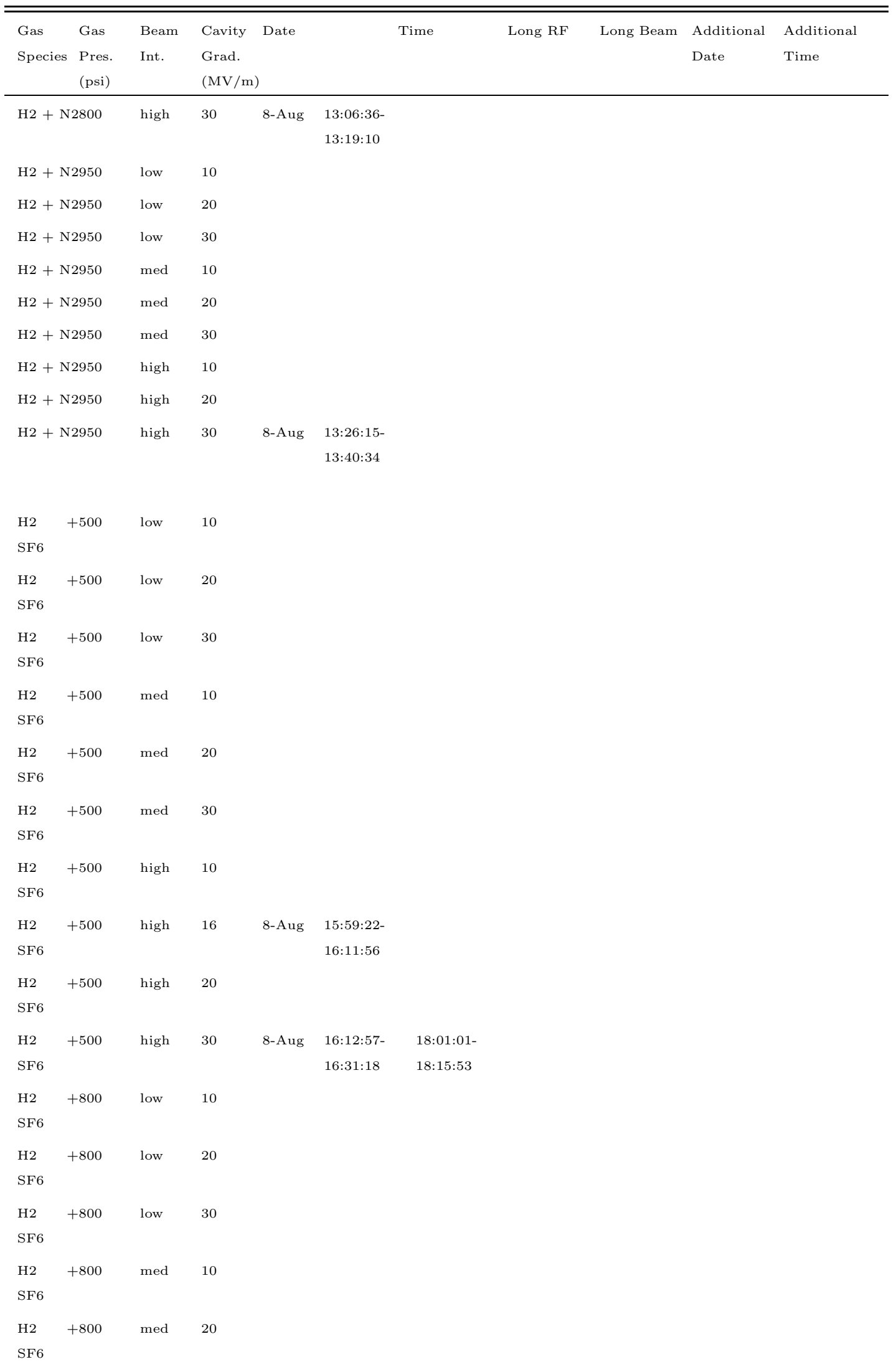




\begin{tabular}{|c|c|c|c|c|c|c|c|c|c|c|}
\hline $\begin{array}{l}\text { Gas } \\
\text { Species }\end{array}$ & $\begin{array}{l}\text { Gas } \\
\text { s Pres. } \\
\text { (psi) }\end{array}$ & $\begin{array}{l}\text { Beam } \\
\text { Int. }\end{array}$ & $\begin{array}{l}\text { Cavity } \\
\text { Grad. } \\
(\mathrm{MV} / \mathrm{m})\end{array}$ & Date & & Time & Long RF & Long Beam & $\begin{array}{l}\text { Additional } \\
\text { Date }\end{array}$ & $\begin{array}{l}\text { Additional } \\
\text { Time }\end{array}$ \\
\hline $\begin{array}{l}\mathrm{H} 2 \\
\mathrm{SF} 6\end{array}$ & +800 & med & 30 & & & & & & & \\
\hline $\begin{array}{l}\mathrm{H} 2 \\
\mathrm{SF} 6\end{array}$ & +800 & high & 10 & & & & & & & \\
\hline $\begin{array}{l}\mathrm{H} 2 \\
\mathrm{SF} 6\end{array}$ & +800 & high & 20 & & & & & & & \\
\hline $\begin{array}{l}\mathrm{H} 2 \\
\mathrm{SF} 6\end{array}$ & +800 & high & 30 & 8-Aug & $\begin{array}{l}16: 41: 48- \\
16: 56: 11\end{array}$ & $\begin{array}{l}17: 38: 57- \\
17: 55: 06\end{array}$ & & & & \\
\hline $\begin{array}{l}\mathrm{H} 2 \\
\mathrm{SF} 6\end{array}$ & +950 & low & 10 & & & & & & & \\
\hline $\begin{array}{l}\mathrm{H} 2 \\
\mathrm{SF} 6\end{array}$ & +950 & low & 20 & & & & & & & \\
\hline $\begin{array}{l}\mathrm{H} 2 \\
\mathrm{SF} 6\end{array}$ & +950 & low & 30 & & & & & & & \\
\hline $\begin{array}{l}\mathrm{H} 2 \\
\mathrm{SF} 6\end{array}$ & +950 & med & 10 & & & & & & & \\
\hline $\begin{array}{l}\mathrm{H} 2 \\
\mathrm{SF} 6\end{array}$ & +950 & med & 20 & & & & & & & \\
\hline $\begin{array}{l}\mathrm{H} 2 \\
\mathrm{SF} 6\end{array}$ & +950 & med & 30 & & & & & & & \\
\hline $\begin{array}{l}\mathrm{H} 2 \\
\mathrm{SF} 6\end{array}$ & +950 & high & 10 & 8-Aug & $\begin{array}{l}17: 20: 07- \\
17: 24: 12\end{array}$ & & & & & \\
\hline $\begin{array}{l}\mathrm{H} 2 \\
\mathrm{SF} 6\end{array}$ & +950 & high & 20 & 8-Aug & $\begin{array}{l}17: 25: 13- \\
17: 27: 15\end{array}$ & & $\begin{array}{l}17: 28: 16- \\
17: 31: 22\end{array}$ & & & \\
\hline $\begin{array}{l}\mathrm{H} 2 \\
\mathrm{SF} 6\end{array}$ & +950 & high & 30 & 8-Aug & $\begin{array}{l}17: 03: 29- \\
17: 19: 06\end{array}$ & & & & & \\
\hline
\end{tabular}


APPENDIX B

RUN CONDITIONS RUN II 
Table B.1. Run conditions for Run II.

\begin{tabular}{|c|c|c|c|c|c|c|c|c|c|c|c|c|}
\hline Date & Run & $\begin{array}{l}\text { Start } \\
\text { Pulse }\end{array}$ & $\begin{array}{l}\text { End } \\
\text { Pulse }\end{array}$ & $\begin{array}{l}\text { Gas } \\
\text { Species }\end{array}$ & $\begin{array}{l}\text { Gas } \\
\text { Conc. } \\
(\%)\end{array}$ & $\begin{array}{l}\text { Gas } \\
\text { Press. } \\
\text { (psi) }\end{array}$ & $\begin{array}{l}\text { Beam } \\
\text { Int. }\end{array}$ & $\begin{array}{l}\text { Beam } \\
\text { Length } \\
(\mu \mathrm{s})\end{array}$ & $\begin{array}{l}\text { Elec. } \\
\text { Field } \\
(\mathrm{MV} / \mathrm{m})\end{array}$ & $\begin{array}{l}\text { Freq. } \\
(\mathrm{MHz})\end{array}$ & $\begin{array}{l}\text { Mag. } \\
\text { Field } \\
(\mathrm{T})\end{array}$ & $\begin{array}{l}\text { Circ. } \\
\text { /Hybr. }\end{array}$ \\
\hline 05-04 & 145 & 3193 & 3206 & Air & 100 & 800 & 1 & 9.5 & 25 & 799.251 & 0 & Hybr. \\
\hline 05-04 & 145 & 3207 & 3217 & Air & 100 & 800 & 1 & 9.5 & 20 & 799.255 & 0 & Hybr. \\
\hline 05-04 & 145 & 3218 & 3225 & Air & 100 & 800 & 1 & 9.5 & 10 & 799.269 & 0 & Hybr. \\
\hline 05-07 & 156 & 3527 & 3537 & $\mathrm{D} 2$ & 100 & 500 & 0.05 & 9.5 & 25 & 807.245 & 0 & Hybr. \\
\hline 05-07 & 156 & 3538 & 3547 & D2 & 100 & 500 & 0.05 & 9.5 & 20 & 807.225 & 0 & Hybr. \\
\hline $05-07$ & 156 & 3548 & 3557 & D2 & 100 & 500 & 0.05 & 9.5 & 10 & 807.233 & 0 & Hybr. \\
\hline 05-07 & 156 & 3558 & 3567 & D2 & 100 & 500 & 0.05 & 9.5 & 5 & 807.245 & 0 & Hybr. \\
\hline 05-07 & 155 & 3487 & 3496 & D2 & 100 & 800 & 0.05 & 9.5 & 25 & 805.449 & 0 & Hybr. \\
\hline 05-07 & 155 & 3497 & 3506 & D2 & 100 & 800 & 0.05 & 9.5 & 20 & 805.433 & 0 & Hybr. \\
\hline 05-07 & 155 & 3507 & 3516 & D2 & 100 & 800 & 0.05 & 9.5 & 10 & 805.444 & 0 & Hybr. \\
\hline 05-07 & 155 & 3517 & 3526 & D2 & 100 & 800 & 0.05 & 9.5 & 5 & 805.452 & 0 & Hybr. \\
\hline 05-07 & 151 & 3417 & 3426 & D2 & 100 & 1140 & 1 & 9.5 & 25 & 803.479 & 0 & Hybr. \\
\hline $05-07$ & 154 & 3428 & 3436 & D2 & 100 & 1140 & 1 & 9.5 & 25 & 803.49 & 0 & Hybr. \\
\hline $05-07$ & 154 & 3444 & 3453 & D2 & 100 & 1140 & 0.05 & 9.5 & 25 & 803.479 & 0 & Hybr. \\
\hline $05-07$ & 154 & 3454 & 3464 & D2 & 100 & 1140 & 0.05 & 9.5 & 20 & 803.484 & 0 & Hybr. \\
\hline $05-07$ & 154 & 3465 & 3475 & D2 & 100 & 1140 & 0.05 & 9.5 & 10 & 803.497 & 0 & Hybr. \\
\hline $05-07$ & 154 & 3477 & 3484 & D2 & 100 & 1140 & 0.05 & 9.5 & 5 & 803.514 & 0 & Hybr. \\
\hline 05-04 & 149 & 3380 & 3389 & $\mathrm{D} 2+\mathrm{A}$ ir & 1 & 300 & 1 & 9.5 & 10 & 808.394 & 0 & Hybr. \\
\hline 05-04 & 149 & 3393 & 3402 & D2+Air & 1 & 300 & 1 & 9.5 & 18 & 808.391 & 0 & Hybr. \\
\hline 05-04 & 148 & 3347 & 3357 & D2+Air & 1 & 800 & 1 & 9.5 & 10 & 805.308 & 0 & Hybr. \\
\hline 05-04 & 148 & 3358 & 3368 & D2+Air & 1 & 800 & 1 & 9.5 & 25 & 805.306 & 0 & Hybr. \\
\hline 05-04 & 148 & 3369 & 3379 & D2+Air & 1 & 800 & 1 & 9.5 & 40 & 805.258 & 0 & Hybr. \\
\hline 05-04 & 147 & 3317 & 3326 & D2+Air & 1 & 1100 & 1 & 9.5 & 10 & 803.531 & 0 & Hybr. \\
\hline 05-04 & 147 & 3327 & 3336 & D2+Air & 1 & 1100 & 1 & 9.5 & 25 & 803.548 & 0 & Hybr. \\
\hline 05-04 & 147 & 3337 & 3346 & D2+Air & 1 & 1100 & 1 & 9.5 & 40 & 803.517 & 0 & Hybr. \\
\hline 05-04 & 146 & 3226 & 3235 & D2+Air & 1 & 1470 & 1 & 9.5 & 25 & 801.565 & 0 & Hybr. \\
\hline 05-04 & 146 & 3236 & 3245 & $\mathrm{D} 2+\mathrm{Air}$ & 1 & 1470 & 1 & 9.5 & 20 & 801.506 & 0 & Hybr. \\
\hline 05-04 & 146 & 3246 & 3249 & D2+Air & 1 & 1470 & 1 & 9.5 & 25 & 801.478 & 0 & Hybr. \\
\hline 05-04 & 146 & 3250 & 3253 & $\mathrm{D} 2+\mathrm{Air}$ & 1 & 1470 & 1 & 9.5 & 20 & 801.467 & 0 & Hybr. \\
\hline 05-04 & 146 & 3257 & 3261 & D2+Air & 1 & 1470 & 1 & 9.5 & 10 & 801.475 & 0 & Hybr. \\
\hline 05-04 & 146 & 3262 & 3274 & D2+Air & 1 & 1470 & 1 & 9.5 & 5 & 801.482 & 0 & Hybr. \\
\hline 05-04 & 146 & 3275 & 3285 & D2+Air & 1 & 1470 & 1 & 9.5 & 40 & 801.476 & 0 & Hybr. \\
\hline 05-04 & 146 & 3286 & 3296 & D2+Air & 1 & 1470 & 0.2 & 9.5 & 10 & 801.465 & 0 & Hybr. \\
\hline 05-04 & 146 & 3297 & 3306 & D2+Air & 1 & 1470 & 0.2 & 9.5 & 25 & 801.502 & 0 & Hybr. \\
\hline 05-04 & 146 & 3307 & 3316 & D2+Air & 1 & 1470 & 0.2 & 9.5 & 40 & 801.474 & 0 & Hybr. \\
\hline $04-21$ & 37 & 478 & 485 & $\mathrm{H} 2$ & 100 & 300 & 1 & 7.5 & 5 & 808.525 & 0 & Circulator \\
\hline $04-21$ & 37 & 487 & 494 & $\mathrm{H} 2$ & 100 & 300 & 1 & 7.5 & 10 & 808.525 & 0 & Circulator \\
\hline $04-21$ & 37 & 495 & 502 & $\mathrm{H} 2$ & 100 & 300 & 1 & 7.5 & 18 & 808.517 & 0 & Circulator \\
\hline
\end{tabular}




\begin{tabular}{|c|c|c|c|c|c|c|c|c|c|c|c|c|}
\hline Date & Run & $\begin{array}{l}\text { Start } \\
\text { Pulse }\end{array}$ & $\begin{array}{l}\text { End } \\
\text { Pulse }\end{array}$ & $\begin{array}{l}\text { Gas } \\
\text { Species }\end{array}$ & $\begin{array}{l}\text { Gas } \\
\text { Conc. } \\
(\%)\end{array}$ & $\begin{array}{l}\text { Gas } \\
\text { Press. } \\
\text { (psi) }\end{array}$ & $\begin{array}{l}\text { Beam } \\
\text { Int. }\end{array}$ & $\begin{array}{l}\text { Beam } \\
\text { Length } \\
(\mu \mathrm{s})\end{array}$ & $\begin{array}{l}\text { Elec. } \\
\text { Field } \\
(\mathrm{MV} / \mathrm{m})\end{array}$ & $\begin{array}{l}\text { Freq. } \\
(\mathrm{MHz})\end{array}$ & $\begin{array}{l}\text { Mag. } \\
\text { Field } \\
(\mathrm{T})\end{array}$ & $\begin{array}{l}\text { Circulator } \\
\text { /Hybr. }\end{array}$ \\
\hline $04-21$ & 39 & 505 & 512 & $\mathrm{H} 2$ & 100 & 300 & 0.2 & 7.5 & 18 & 808.517 & 0 & Circulator \\
\hline $04-21$ & 39 & 540 & 546 & $\mathrm{H} 2$ & 100 & 300 & 0.2 & 7.5 & 10 & 808.51 & 0 & Circulator \\
\hline $04-21$ & 39 & 548 & 555 & $\mathrm{H} 2$ & 100 & 300 & 0.2 & 7.5 & 5 & 808.52 & 0 & Circulator \\
\hline $04-21$ & 39 & 557 & 564 & $\mathrm{H} 2$ & 100 & 300 & 0.1 & 7.5 & 5 & 808.522 & 0 & Circulator \\
\hline $04-21$ & 39 & 566 & 573 & $\mathrm{H} 2$ & 100 & 300 & 0.1 & 7.5 & 10 & 808.525 & 0 & Circulator \\
\hline $04-21$ & 39 & 575 & 582 & $\mathrm{H} 2$ & 100 & 300 & 0.1 & 7.5 & 18 & 808.521 & 0 & Circulator \\
\hline $04-23$ & 55 & 275 & 284 & $\mathrm{H} 2$ & 100 & 300 & 0.05 & 7.5 & 5 & 808.491 & 0 & Circulator \\
\hline $04-23$ & 55 & 286 & 295 & $\mathrm{H} 2$ & 100 & 300 & 0.05 & 7.5 & 10 & 808.492 & 0 & Circulator \\
\hline $04-23$ & 55 & 296 & 306 & $\mathrm{H} 2$ & 100 & 300 & 0.05 & 7.5 & 18 & 808.489 & 0 & Circulator \\
\hline 05-02 & 125 & 2009 & 2019 & $\mathrm{H} 2$ & 100 & 300 & 1 & 9.5 & 10 & 808.421 & 3 & Hybr. \\
\hline 05-02 & 125 & 2020 & 2030 & $\mathrm{H} 2$ & 100 & 300 & 1 & 9.5 & 5 & 808.426 & 3 & Hybr. \\
\hline 05-02 & 125 & 2031 & 2040 & $\mathrm{H} 2$ & 100 & 300 & 1 & 9.5 & 15 & 808.432 & 3 & Hybr. \\
\hline \multirow[t]{2}{*}{ 05-02 } & 125 & 2041 & 2050 & $\mathrm{H} 2$ & 100 & 300 & 1 & 9.5 & 10 & 808.432 & 3 & Hybr. \\
\hline & & & & & & & & & & & 2.54 & \\
\hline 05-02 & 125 & 2051 & 2059 & $\mathrm{H} 2$ & 100 & 300 & 1 & 9.5 & 10 & 808.432 & 2.5 & Hybr. \\
\hline \multirow[t]{2}{*}{ 05-02 } & 125 & 2060 & 2071 & $\mathrm{H} 2$ & 100 & 300 & 1 & 9.5 & 10 & 808.438 & 2.46 & Hybr. \\
\hline & & & & & & & & & & & 2.04 & \\
\hline 05-02 & 125 & 2072 & 2079 & $\mathrm{H} 2$ & 100 & 300 & 1 & 9.5 & 10 & 808.432 & 2 & Hybr. \\
\hline \multirow[t]{2}{*}{ 05-02 } & 125 & 2080 & 2095 & $\mathrm{H} 2$ & 100 & 300 & 1 & 9.5 & 10 & 808.438 & 2 & Hybr. \\
\hline & & & & & & & & & & & 1.54 & \\
\hline 05-02 & 125 & 2096 & 2103 & $\mathrm{H} 2$ & 100 & 300 & 1 & 9.5 & 10 & 808.432 & 1.5 & Hybr. \\
\hline \multirow[t]{2}{*}{ 05-02 } & 125 & 2104 & 2121 & $\mathrm{H} 2$ & 100 & 300 & 1 & 9.5 & 10 & 808.438 & 1.5 & Hybr. \\
\hline & & & & & & & & & & & 1.04 & \\
\hline 05-02 & 125 & 2122 & 2129 & $\mathrm{H} 2$ & 100 & 300 & 1 & 9.5 & 10 & 808.432 & 1 & Hybr. \\
\hline \multirow[t]{2}{*}{ 05-02 } & 125 & 2130 & 2151 & $\mathrm{H} 2$ & 100 & 300 & 1 & 9.5 & 10 & 808.438 & 1 & Hybr. \\
\hline & & & & & & & & & & & 0.54 & \\
\hline 05-02 & 125 & 2152 & 2161 & $\mathrm{H} 2$ & 100 & 300 & 1 & 9.5 & 10 & 808.432 & 0.5 & Hybr. \\
\hline \multirow[t]{2}{*}{ 05-02 } & 125 & 2162 & 2184 & $\mathrm{H} 2$ & 100 & 300 & 1 & 9.5 & 10 & 808.454 & 0.41 & Hybr. \\
\hline & & & & & & & & & & & 0 & \\
\hline 05-02 & 125 & 2185 & 2192 & $\mathrm{H} 2$ & 100 & 300 & 1 & 9.5 & 10 & 808.432 & 0 & Hybr. \\
\hline $04-17$ & 12 & & & $\mathrm{H} 2$ & 100 & 500 & & 9.5 & & 807.36 & 0 & Circulator \\
\hline $04-17$ & 13 & & & $\mathrm{H} 2$ & 100 & 500 & & 9.5 & 10 & 807.358 & 0 & Circulator \\
\hline $04-17$ & 14 & 7 & 100 & $\mathrm{H} 2$ & 100 & 500 & & 9.5 & 17.4 & 807.334 & 0 & Circulator \\
\hline $04-21$ & 41 & 583 & 590 & $\mathrm{H} 2$ & 100 & 500 & 0.1 & 7.5 & 5 & 807.245 & 0 & Circulator \\
\hline $04-21$ & 41 & 596 & 603 & $\mathrm{H} 2$ & 100 & 500 & 0.1 & 7.5 & 10 & 807.25 & 0 & Circulator \\
\hline $04-21$ & 41 & 604 & 611 & $\mathrm{H} 2$ & 100 & 500 & 0.1 & 7.5 & 20 & 807.246 & 0 & Circulator \\
\hline $04-21$ & 41 & 612 & 619 & $\mathrm{H} 2$ & 100 & 500 & 0.1 & 7.5 & 25 & 807.231 & 0 & Circulator \\
\hline $04-21$ & 41 & 620 & 627 & $\mathrm{H} 2$ & 100 & 500 & 0.2 & 7.5 & 25 & 807.221 & 0 & Circulator \\
\hline $04-21$ & 41 & 630 & 637 & $\mathrm{H} 2$ & 100 & 500 & 0.2 & 7.5 & 20 & 807.229 & 0 & Circulator \\
\hline $04-21$ & 41 & 638 & 645 & $\mathrm{H} 2$ & 100 & 500 & 0.2 & 7.5 & 10 & 807.235 & 0 & Circulator \\
\hline $04-21$ & 41 & 646 & 653 & $\mathrm{H} 2$ & 100 & 500 & 0.2 & 7.5 & 5 & 807.244 & 0 & Circulator \\
\hline
\end{tabular}




\begin{tabular}{|c|c|c|c|c|c|c|c|c|c|c|c|c|}
\hline Date & Run & $\begin{array}{l}\text { Start } \\
\text { Pulse }\end{array}$ & $\begin{array}{l}\text { End } \\
\text { Pulse }\end{array}$ & $\begin{array}{l}\text { Gas } \\
\text { Species }\end{array}$ & $\begin{array}{l}\text { Gas } \\
\text { Conc. } \\
(\%)\end{array}$ & $\begin{array}{l}\text { Gas } \\
\text { Press. } \\
\text { (psi) }\end{array}$ & $\begin{array}{l}\text { Beam } \\
\text { Int. }\end{array}$ & $\begin{array}{l}\text { Beam } \\
\text { Length } \\
(\mu \mathrm{s})\end{array}$ & $\begin{array}{l}\text { Elec. } \\
\text { Field } \\
(\mathrm{MV} / \mathrm{m})\end{array}$ & $\begin{array}{l}\text { Freq. } \\
(\mathrm{MHz})\end{array}$ & $\begin{array}{l}\text { Mag. } \\
\text { Field } \\
(\mathrm{T})\end{array}$ & $\begin{array}{l}\text { Circulator } \\
\text { /Hybr. }\end{array}$ \\
\hline $04-21$ & 41 & 654 & 661 & $\mathrm{H} 2$ & 100 & 500 & 1 & 7.5 & 5 & 807.249 & 0 & Circulator \\
\hline $04-21$ & 41 & 662 & 669 & $\mathrm{H} 2$ & 100 & 500 & 1 & 7.5 & 10 & 807.254 & 0 & Circulator \\
\hline 04-21 & 41 & 670 & 677 & $\mathrm{H} 2$ & 100 & 500 & 1 & 7.5 & 20 & 807.25 & 0 & Circulator \\
\hline $04-21$ & 41 & 678 & 685 & $\mathrm{H} 2$ & 100 & 500 & 1 & 7.5 & 25 & 807.254 & 0 & Circulator \\
\hline $04-23$ & 54 & 237 & 245 & $\mathrm{H} 2$ & 100 & 500 & 0.05 & 7.5 & 5 & 807.24 & 0 & Circulator \\
\hline $04-23$ & 54 & 246 & 254 & $\mathrm{H} 2$ & 100 & 500 & 0.05 & 7.5 & 10 & 807.238 & 0 & Circulator \\
\hline $04-23$ & 54 & 255 & 264 & $\mathrm{H} 2$ & 100 & 500 & 0.05 & 7.5 & 20 & 807.234 & 0 & Circulator \\
\hline 04-23 & 54 & 265 & 274 & $\mathrm{H} 2$ & 100 & 500 & 0.05 & 7.5 & 25 & 807.221 & 0 & Circulator \\
\hline 05-07 & 159 & 3648 & 3657 & $\mathrm{H} 2$ & 100 & 500 & 0.05 & 9.5 & 25 & 807.211 & 0 & Hybr. \\
\hline 05-07 & 159 & 3658 & 3667 & $\mathrm{H} 2$ & 100 & 500 & 0.05 & 9.5 & 20 & 807.211 & 0 & Hybr. \\
\hline 05-07 & 159 & 3668 & 3677 & $\mathrm{H} 2$ & 100 & 500 & 0.05 & 9.5 & 10 & 807.219 & 0 & Hybr. \\
\hline 05-07 & 159 & 3678 & 3687 & $\mathrm{H} 2$ & 100 & 500 & 0.05 & 9.5 & 5 & 807.235 & 0 & Hybr. \\
\hline $04-18$ & 16 & & & $\mathrm{H} 2$ & 100 & 800 & & 9.5 & 29.2 & 805.482 & 0 & Circulator \\
\hline $04-18$ & 17 & 25 & 33 & $\mathrm{H} 2$ & 100 & 800 & 1 & 9.5 & 27.7 & 805.47 & 0 & Circulator \\
\hline $04-18$ & 17 & 36 & 44 & $\mathrm{H} 2$ & 100 & 800 & 1 & 9.5 & 20 & 805.48 & 0 & Circulator \\
\hline $04-18$ & 17 & 47 & 58 & $\mathrm{H} 2$ & 100 & 800 & 1 & 9.5 & 10 & 805.5 & 0 & Circulator \\
\hline 04-18 & 17 & 60 & 69 & $\mathrm{H} 2$ & 100 & 800 & 1 & 9.5 & 5 & 805.51 & 0 & Circulator \\
\hline $04-18$ & 18 & & & $\mathrm{H} 2$ & 100 & 800 & & 9.5 & & 805.535 & 0 & Circulator \\
\hline 04-18 & 19 & & & $\mathrm{H} 2$ & 100 & 800 & & 9.5 & & 805.535 & 0 & Circulator \\
\hline 04-18 & 20 & & & $\mathrm{H} 2$ & 100 & 800 & & 9.5 & & 805.535 & 0 & Circulator \\
\hline 04-18 & 22 & & & $\mathrm{H} 2$ & 100 & 800 & & 9.5 & & 805.535 & 0 & Circulator \\
\hline $04-18$ & 23 & 0 & 7 & $\mathrm{H} 2$ & 100 & 800 & 0.2 & 9.5 & 5 & 805.55 & 0 & Circulator \\
\hline 04-18 & 23 & 9 & 16 & $\mathrm{H} 2$ & 100 & 800 & 0.2 & 9.5 & 10 & 805.54 & 0 & Circulator \\
\hline 04-18 & 23 & 17 & 27 & $\mathrm{H} 2$ & 100 & 800 & 0.2 & 9.5 & 20 & 805.55 & 0 & Circulator \\
\hline 04-18 & 23 & 28 & 40 & $\mathrm{H} 2$ & 100 & 800 & 0.2 & 9.5 & 27.7 & 805.57 & 0 & Circulator \\
\hline 04-18 & 24 & 3 & 11 & $\mathrm{H} 2$ & 100 & 800 & 0.1 & 9.5 & 27.7 & 805.55 & 0 & Circulator \\
\hline $04-18$ & 24 & 13 & 23 & $\mathrm{H} 2$ & 100 & 800 & 0.1 & 9.5 & 20 & 805.54 & 0 & Circulator \\
\hline $04-18$ & 24 & 24 & 33 & $\mathrm{H} 2$ & 100 & 800 & 0.1 & 9.5 & 10 & 805.55 & 0 & Circulator \\
\hline 04-18 & 24 & 34 & 43 & $\mathrm{H} 2$ & 100 & 800 & 0.1 & 9.5 & 5 & 805.57 & 0 & Circulator \\
\hline $04-18$ & 24 & 44 & 48 & $\mathrm{H} 2$ & 100 & 800 & 0.05 & 9.5 & 10 & 805.57 & 0 & Circulator \\
\hline 04-18 & 24 & 49 & 54 & $\mathrm{H} 2$ & 100 & 800 & 0.05 & 9.5 & 20 & 805.58 & 0 & Circulator \\
\hline 04-18 & 24 & 55 & 59 & $\mathrm{H} 2$ & 100 & 800 & 0.05 & 9.5 & 27.7 & 805.56 & 0 & Circulator \\
\hline $04-22$ & 42 & 686 & 692 & $\mathrm{H} 2$ & 100 & 800 & 1 & 7.5 & 5 & 805.463 & 0 & Circulator \\
\hline $04-22$ & 42 & 693 & 700 & $\mathrm{H} 2$ & 100 & 800 & 1 & 7.5 & 10 & 805.466 & 0 & Circulator \\
\hline $04-22$ & 42 & 701 & 708 & $\mathrm{H} 2$ & 100 & 800 & 1 & 7.5 & 20 & 805.465 & 0 & Circulator \\
\hline $04-22$ & 42 & 709 & 716 & $\mathrm{H} 2$ & 100 & 800 & 1 & 7.5 & 25 & 805.458 & 0 & Circulator \\
\hline $04-22$ & 42 & 720 & 727 & $\mathrm{H} 2$ & 100 & 800 & 0.2 & 7.5 & 25 & 805.458 & 0 & Circulator \\
\hline $04-22$ & 42 & 728 & 735 & $\mathrm{H} 2$ & 100 & 800 & 0.2 & 7.5 & 20 & 805.456 & 0 & Circulator \\
\hline $04-22$ & 42 & 736 & 743 & $\mathrm{H} 2$ & 100 & 800 & 0.2 & 7.5 & 10 & 805.47 & 0 & Circulator \\
\hline $04-22$ & 42 & 744 & 751 & $\mathrm{H} 2$ & 100 & 800 & 0.2 & 7.5 & 5 & 805.482 & 0 & Circulator \\
\hline
\end{tabular}




\begin{tabular}{|c|c|c|c|c|c|c|c|c|c|c|c|c|}
\hline Date & Run & $\begin{array}{l}\text { Start } \\
\text { Pulse }\end{array}$ & $\begin{array}{l}\text { End } \\
\text { Pulse }\end{array}$ & $\begin{array}{l}\text { Gas } \\
\text { Species }\end{array}$ & $\begin{array}{l}\text { Gas } \\
\text { Conc. } \\
(\%)\end{array}$ & $\begin{array}{l}\text { Gas } \\
\text { Press. } \\
\text { (psi) }\end{array}$ & $\begin{array}{l}\text { Beam } \\
\text { Int. }\end{array}$ & $\begin{array}{l}\text { Beam } \\
\text { Length } \\
(\mu \mathrm{s})\end{array}$ & $\begin{array}{l}\text { Elec. } \\
\text { Field } \\
(\mathrm{MV} / \mathrm{m})\end{array}$ & $\begin{array}{l}\text { Freq. } \\
(\mathrm{MHz})\end{array}$ & $\begin{array}{l}\text { Mag. } \\
\text { Field } \\
(\mathrm{T})\end{array}$ & $\begin{array}{l}\text { Circulator } \\
\text { /Hybr. }\end{array}$ \\
\hline $04-22$ & 42 & 754 & 761 & $\mathrm{H} 2$ & 100 & 800 & 0.1 & 7.5 & 5 & 805.482 & 0 & Circulator \\
\hline $04-22$ & 42 & 762 & 769 & $\mathrm{H} 2$ & 100 & 800 & 0.1 & 7.5 & 10 & 805.495 & 0 & Circulator \\
\hline $04-22$ & 42 & 771 & 778 & $\mathrm{H} 2$ & 100 & 800 & 0.1 & 7.5 & 20 & 805.496 & 0 & Circulator \\
\hline $04-22$ & 42 & 780 & 787 & $\mathrm{H} 2$ & 100 & 800 & 0.1 & 7.5 & 25 & 805.486 & 0 & Circulator \\
\hline $04-23$ & 51 & 191 & 204 & $\mathrm{H} 2$ & 100 & 800 & 0.05 & 7.5 & 25 & 805.413 & 0 & Circulator \\
\hline $04-23$ & 51 & 205 & 212 & $\mathrm{H} 2$ & 100 & 800 & 0.05 & 7.5 & 20 & 805.396 & 0 & Circulator \\
\hline $04-23$ & 51 & 213 & 225 & $\mathrm{H} 2$ & 100 & 800 & 0.05 & 7.5 & 10 & 805.4 & 0 & Circulator \\
\hline $04-23$ & 53 & 226 & 226 & $\mathrm{H} 2$ & 100 & 800 & 0.05 & 7.5 & 10 & 805.4 & 0 & Circulator \\
\hline $04-23$ & 53 & 227 & 236 & $\mathrm{H} 2$ & 100 & 800 & 0.05 & 7.5 & 5 & 805.414 & 0 & Circulator \\
\hline 05-02 & 124 & 1965 & 1976 & $\mathrm{H} 2$ & 100 & 800 & 1 & 9.5 & 40 & 805.4 & 3 & Hybr. \\
\hline 05-02 & 124 & 1977 & 1988 & $\mathrm{H} 2$ & 100 & 800 & 1 & 9.5 & 25 & 805.401 & 3 & Hybr. \\
\hline 05-02 & 124 & 1989 & 1998 & $\mathrm{H} 2$ & 100 & 800 & 1 & 9.5 & 20 & 805.419 & 3 & Hybr. \\
\hline 05-02 & 124 & 1999 & 2008 & $\mathrm{H} 2$ & 100 & 800 & 1 & 9.5 & 10 & 805.434 & 3 & Hybr. \\
\hline $05-07$ & 158 & 3608 & 3617 & $\mathrm{H} 2$ & 100 & 800 & 0.05 & 9.5 & 25 & 805.384 & 0 & Hybr. \\
\hline $05-07$ & 158 & 3618 & 3627 & $\mathrm{H} 2$ & 100 & 800 & 0.05 & 9.5 & 20 & 805.373 & 0 & Hybr. \\
\hline 05-07 & 158 & 3628 & 3637 & $\mathrm{H} 2$ & 100 & 800 & 0.05 & 9.5 & 10 & 805.383 & 0 & Hybr. \\
\hline 05-07 & 158 & 3638 & 3647 & $\mathrm{H} 2$ & 100 & 800 & 0.05 & 9.5 & 5 & 805.398 & 0 & Hybr. \\
\hline 04-20 & 35 & 252 & 261 & $\mathrm{H} 2$ & 100 & 950 & 1 & 7.5 & 25 & 804.562 & 0 & Circulator \\
\hline $04-20$ & 35 & 263 & 271 & $\mathrm{H} 2$ & 100 & 950 & 1 & 7.5 & 20 & 804.57 & 0 & Circulator \\
\hline $04-20$ & 35 & 272 & 279 & $\mathrm{H} 2$ & 100 & 950 & 1 & 7.5 & 10 & 804.578 & 0 & Circulator \\
\hline $04-20$ & 35 & 281 & 289 & $\mathrm{H} 2$ & 100 & 950 & 1 & 7.5 & 5 & 804.598 & 0 & Circulator \\
\hline $04-20$ & 35 & 294 & 302 & $\mathrm{H} 2$ & 100 & 950 & 0.2 & 7.5 & 5 & 804.598 & 0 & Circulator \\
\hline $04-20$ & 35 & 305 & 313 & $\mathrm{H} 2$ & 100 & 950 & 0.2 & 7.5 & 10 & 804.617 & 0 & Circulator \\
\hline $04-20$ & 35 & 315 & 322 & $\mathrm{H} 2$ & 100 & 950 & 0.2 & 7.5 & 20 & 804.619 & 0 & Circulator \\
\hline $04-20$ & 35 & 323 & 330 & $\mathrm{H} 2$ & 100 & 950 & 0.2 & 7.5 & 25 & 804.61 & 0 & Circulator \\
\hline $04-20$ & 35 & 334 & 341 & $\mathrm{H} 2$ & 100 & 950 & 0.1 & 7.5 & 25 & 804.61 & 0 & Circulator \\
\hline $04-20$ & 35 & 342 & 349 & $\mathrm{H} 2$ & 100 & 950 & 0.1 & 7.5 & 20 & 804.611 & 0 & Circulator \\
\hline $04-20$ & 35 & 350 & 357 & $\mathrm{H} 2$ & 100 & 950 & 0.1 & 7.5 & 10 & 804.625 & 0 & Circulator \\
\hline $04-20$ & 35 & 358 & 365 & $\mathrm{H} 2$ & 100 & 950 & 0.1 & 7.5 & 5 & 804.642 & 0 & Circulator \\
\hline $04-23$ & 50 & 150 & 159 & $\mathrm{H} 2$ & 100 & 950 & 0.05 & 7.5 & 5 & 804.628 & 0 & Circulator \\
\hline $04-23$ & 50 & 160 & 171 & $\mathrm{H} 2$ & 100 & 950 & 0.05 & 7.5 & 10 & 804.637 & 0 & Circulator \\
\hline $04-23$ & 50 & 172 & 181 & $\mathrm{H} 2$ & 100 & 950 & 0.05 & 7.5 & 20 & 804.634 & 0 & Circulator \\
\hline $04-23$ & 50 & 182 & 190 & $\mathrm{H} 2$ & 100 & 950 & 0.05 & 7.5 & 25 & 804.629 & 0 & Circulator \\
\hline 05-01 & 113 & 1718 & 1727 & $\mathrm{H} 2$ & 100 & 950 & 0.1 & 9.5 & 40 & 804.575 & 0 & Hybr. \\
\hline 05-01 & 113 & 1728 & 1739 & $\mathrm{H} 2$ & 100 & 950 & 0.1 & 9.5 & 30 & 804.545 & 0 & Hybr. \\
\hline 05-01 & 113 & 1740 & 1752 & $\mathrm{H} 2$ & 100 & 950 & 1 & 9.5 & 30 & 804.563 & 0 & Hybr. \\
\hline 05-01 & 113 & 1753 & 1762 & $\mathrm{H} 2$ & 100 & 950 & 1 & 9.5 & 40 & 804.577 & 0 & Hybr. \\
\hline 04-19 & 29 & 17 & 26 & $\mathrm{H} 2$ & 100 & 1100 & 1 & 7.5 & 25 & 803.67 & 0 & Circulator \\
\hline 04-19 & 29 & 28 & 37 & $\mathrm{H} 2$ & 100 & 1100 & 1 & 7.5 & 20 & 803.67 & 0 & Circulator \\
\hline 04-19 & 31 & 40 & 47 & $\mathrm{H} 2$ & 100 & 1100 & 1 & 7.5 & 10 & 803.73 & 0 & Circulator \\
\hline
\end{tabular}




\begin{tabular}{|c|c|c|c|c|c|c|c|c|c|c|c|c|}
\hline Date & Run & $\begin{array}{l}\text { Start } \\
\text { Pulse }\end{array}$ & $\begin{array}{l}\text { End } \\
\text { Pulse }\end{array}$ & $\begin{array}{l}\text { Gas } \\
\text { Species }\end{array}$ & $\begin{array}{l}\text { Gas } \\
\text { Conc. } \\
(\%)\end{array}$ & $\begin{array}{l}\text { Gas } \\
\text { Press. } \\
\text { (psi) }\end{array}$ & $\begin{array}{l}\text { Beam } \\
\text { Int. }\end{array}$ & $\begin{array}{l}\text { Beam } \\
\text { Length } \\
(\mu \mathrm{s})\end{array}$ & $\begin{array}{l}\text { Elec. } \\
\text { Field } \\
(\mathrm{MV} / \mathrm{m})\end{array}$ & $\begin{array}{l}\text { Freq. } \\
(\mathrm{MHz})\end{array}$ & $\begin{array}{l}\text { Mag. } \\
\text { Field } \\
(\mathrm{T})\end{array}$ & $\begin{array}{l}\text { Circulator } \\
\text { /Hybr. }\end{array}$ \\
\hline 04-19 & 31 & 48 & 54 & $\mathrm{H} 2$ & 100 & 1100 & 1 & 7.5 & 5 & 803.74 & 0 & Circulator \\
\hline 04-19 & 31 & 55 & 62 & $\mathrm{H} 2$ & 100 & 1100 & 0.2 & 7.5 & 5 & 803.74 & 0 & Circulator \\
\hline 04-19 & 31 & 65 & 73 & $\mathrm{H} 2$ & 100 & 1100 & 0.2 & 7.5 & 10 & 803.76 & 0 & Circulator \\
\hline 04-19 & 31 & 75 & 82 & $\mathrm{H} 2$ & 100 & 1100 & 0.2 & 7.5 & 20 & 803.76 & 0 & Circulator \\
\hline 04-19 & 31 & 83 & 90 & $\mathrm{H} 2$ & 100 & 1100 & 0.2 & 7.5 & 25 & 803.76 & 0 & Circulator \\
\hline 04-19 & 31 & 101 & 108 & $\mathrm{H} 2$ & 100 & 1100 & 0.1 & 7.5 & 25 & 803.757 & 0 & Circulator \\
\hline 04-19 & 31 & 109 & 116 & $\mathrm{H} 2$ & 100 & 1100 & 0.1 & 7.5 & 20 & 803.76 & 0 & Circulator \\
\hline 04-19 & 31 & 117 & 125 & $\mathrm{H} 2$ & 100 & 1100 & 0.1 & 7.5 & 10 & 803.78 & 0 & Circulator \\
\hline 04-19 & 31 & 126 & 133 & $\mathrm{H} 2$ & 100 & 1100 & 0.1 & 7.5 & 5 & 803.79 & 0 & Circulator \\
\hline $04-23$ & 48 & & & $\mathrm{H} 2$ & 100 & 1100 & 0.05 & 7.5 & & 803.71 & 0 & Circulator \\
\hline $04-23$ & 49 & 111 & 122 & $\mathrm{H} 2$ & 100 & 1100 & 0.05 & 7.5 & 25 & 803.71 & 0 & Circulator \\
\hline $04-23$ & 49 & 123 & 131 & $\mathrm{H} 2$ & 100 & 1100 & 0.05 & 7.5 & 20 & 803.703 & 0 & Circulator \\
\hline $04-23$ & 49 & 132 & 140 & $\mathrm{H} 2$ & 100 & 1100 & 0.05 & 7.5 & 10 & 803.719 & 0 & Circulator \\
\hline $04-23$ & 49 & 141 & 149 & $\mathrm{H} 2$ & 100 & 1100 & 0.05 & 7.5 & 5 & 803.732 & 0 & Circulator \\
\hline $04-26$ & 88 & 1530 & 1540 & $\mathrm{H} 2$ & 100 & 1100 & 1 & 9.5 & 25 & 803.679 & 0 & Circulator \\
\hline $04-26$ & 91 & 1548 & 1552 & $\mathrm{H} 2$ & 100 & 1100 & 1 & 9.5 & 25 & 803.783 & 0 & Circulator \\
\hline 05-01 & 112 & 1555 & 1564 & $\mathrm{H} 2$ & 100 & 1100 & 1 & 9.5 & 40 & 803.529 & 0 & Hybr. \\
\hline 05-01 & 112 & 1565 & 1704 & $\mathrm{H} 2$ & 100 & 1100 & 1 & 9.5 & 30 & 803.557 & 0 & Hybr. \\
\hline 05-01 & 112 & 1708 & 1717 & $\mathrm{H} 2$ & 100 & 1100 & 0.1 & 9.5 & 40 & 803.564 & 0 & Hybr. \\
\hline $05-07$ & 157 & 3568 & 3577 & $\mathrm{H} 2$ & 100 & 1140 & 0.05 & 9.5 & 25 & 803.485 & 0 & Hybr. \\
\hline $05-07$ & 157 & 3578 & 3587 & $\mathrm{H} 2$ & 100 & 1140 & 0.05 & 9.5 & 20 & 803.483 & 0 & Hybr. \\
\hline 05-07 & 157 & 3588 & 3597 & $\mathrm{H} 2$ & 100 & 1140 & 0.05 & 9.5 & 10 & 803.495 & 0 & Hybr. \\
\hline $05-07$ & 157 & 3598 & 3607 & $\mathrm{H} 2$ & 100 & 1140 & 0.05 & 9.5 & 5 & 803.509 & 0 & Hybr. \\
\hline $04-20$ & 36 & 367 & 374 & $\mathrm{H} 2$ & 100 & 1300 & 0.1 & 7.5 & 5 & 802.533 & 0 & Circulator \\
\hline $04-20$ & 36 & 377 & 384 & $\mathrm{H} 2$ & 100 & 1300 & 0.1 & 7.5 & 10 & 802.546 & 0 & Circulator \\
\hline $04-20$ & 36 & 385 & 392 & $\mathrm{H} 2$ & 100 & 1300 & 0.1 & 7.5 & 20 & 802.553 & 0 & Circulator \\
\hline $04-20$ & 36 & 393 & 401 & $\mathrm{H} 2$ & 100 & 1300 & 0.1 & 7.5 & 25 & 802.549 & 0 & Circulator \\
\hline $04-20$ & 36 & 409 & 416 & $\mathrm{H} 2$ & 100 & 1300 & 0.2 & 7.5 & 25 & 802.55 & 0 & Circulator \\
\hline $04-20$ & 36 & 417 & 424 & $\mathrm{H} 2$ & 100 & 1300 & 0.2 & 7.5 & 20 & 802.56 & 0 & Circulator \\
\hline $04-20$ & 36 & 425 & 432 & $\mathrm{H} 2$ & 100 & 1300 & 0.2 & 7.5 & 10 & 802.576 & 0 & Circulator \\
\hline $04-20$ & 36 & 433 & 440 & $\mathrm{H} 2$ & 100 & 1300 & 0.2 & 7.5 & 5 & 802.594 & 0 & Circulator \\
\hline $04-20$ & 36 & 445 & 452 & $\mathrm{H} 2$ & 100 & 1300 & 1 & 7.5 & 5 & 802.61 & 0 & Circulator \\
\hline $04-20$ & 36 & 453 & 461 & $\mathrm{H} 2$ & 100 & 1300 & 1 & 7.5 & 10 & 802.618 & 0 & Circulator \\
\hline $04-20$ & 36 & 462 & 469 & $\mathrm{H} 2$ & 100 & 1300 & 1 & 7.5 & 20 & 802.623 & 0 & Circulator \\
\hline $04-20$ & 36 & 470 & 477 & $\mathrm{H} 2$ & 100 & 1300 & 1 & 7.5 & 25 & 802.619 & 0 & Circulator \\
\hline $04-23$ & 45 & 63 & 64 & $\mathrm{H} 2$ & 100 & 1300 & 0.05 & 7.5 & 5 & 802.518 & 0 & Circulator \\
\hline $04-23$ & 47 & 69 & 78 & $\mathrm{H} 2$ & 100 & 1300 & 0.05 & 7.5 & 5 & 802.527 & 0 & Circulator \\
\hline $04-23$ & 47 & 80 & 88 & $\mathrm{H} 2$ & 100 & 1300 & 0.05 & 7.5 & 10 & 802.551 & 0 & Circulator \\
\hline $04-23$ & 47 & 89 & 97 & $\mathrm{H} 2$ & 100 & 1300 & 0.05 & 7.5 & 20 & 802.558 & 0 & Circulator \\
\hline $04-23$ & 47 & 98 & 106 & $\mathrm{H} 2$ & 100 & 1300 & 0.05 & 7.5 & 25 & 802.559 & 0 & Circulator \\
\hline
\end{tabular}




\begin{tabular}{|c|c|c|c|c|c|c|c|c|c|c|c|c|}
\hline Date & Run & $\begin{array}{l}\text { Start } \\
\text { Pulse }\end{array}$ & $\begin{array}{l}\text { End } \\
\text { Pulse }\end{array}$ & $\begin{array}{l}\text { Gas } \\
\text { Species }\end{array}$ & $\begin{array}{l}\text { Gas } \\
\text { Conc. } \\
(\%)\end{array}$ & $\begin{array}{l}\text { Gas } \\
\text { Press. } \\
\text { (psi) }\end{array}$ & $\begin{array}{l}\text { Beam } \\
\text { Int. }\end{array}$ & $\begin{array}{l}\text { Beam } \\
\text { Length } \\
(\mu \mathrm{s})\end{array}$ & $\begin{array}{l}\text { Elec. } \\
\text { Field } \\
(\mathrm{MV} / \mathrm{m})\end{array}$ & $\begin{array}{l}\text { Freq. } \\
(\mathrm{MHz})\end{array}$ & $\begin{array}{l}\text { Mag. } \\
\text { Field } \\
(\mathrm{T})\end{array}$ & $\begin{array}{l}\text { Circulator } \\
\text { /Hybr. }\end{array}$ \\
\hline $04-23$ & 43 & 1 & 12 & $\mathrm{H} 2$ & 100 & 1450 & 0.05 & 7.5 & 25 & 801.65 & 0 & Circulator \\
\hline $04-26$ & 92 & 1555 & 1562 & $\mathrm{H} 2$ & 100 & 1470 & 1 & 9.5 & 25 & 801.635 & 0 & Circulator \\
\hline $04-27$ & 104 & 1773 & 1782 & $\mathrm{H} 2$ & 100 & 1470 & 1 & 9.5 & 25 & 801.629 & 0 & Circulator \\
\hline $04-27$ & 104 & 1783 & 1793 & $\mathrm{H} 2$ & 100 & 1470 & 1 & 9.5 & 20 & 801.63 & 0 & Circulator \\
\hline $04-27$ & 104 & 1794 & 1803 & $\mathrm{H} 2$ & 100 & 1470 & 1 & 9.5 & 10 & 801.65 & 0 & Circulator \\
\hline $04-27$ & 104 & 1804 & 1813 & $\mathrm{H} 2$ & 100 & 1470 & 1 & 9.5 & 5 & 801.673 & 0 & Circulator \\
\hline 04-30 & 107 & 1814 & 1824 & $\mathrm{H} 2$ & 100 & 1470 & 1 & 9.5 & 50 & 801.646 & 0 & Hybr. \\
\hline 04-30 & 107 & 1825 & 1842 & $\mathrm{H} 2$ & 100 & 1470 & 1 & 9.5 & 40 & 801.637 & 0 & Hybr. \\
\hline 04-30 & 107 & 1843 & 1861 & $\mathrm{H} 2$ & 100 & 1470 & 1 & 9.5 & 40 & 801.708 & 0 & Hybr. \\
\hline 04-30 & 107 & 1862 & 1874 & $\mathrm{H} 2$ & 100 & 1470 & 0.1 & 9.5 & 40 & 801.751 & 0 & Hybr. \\
\hline 04-30 & 107 & 1876 & 1887 & $\mathrm{H} 2$ & 100 & 1470 & 0.1 & 9.5 & 50 & 801.761 & 0 & Hybr. \\
\hline 04-30 & 109 & 1888 & 1889 & $\mathrm{H} 2$ & 100 & 1470 & 0.1 & 9.5 & 50 & 801.761 & 0 & Hybr. \\
\hline 04-30 & 111 & 1890 & 1894 & $\mathrm{H} 2$ & 100 & 1470 & 0.1 & 9.5 & 50 & 801.761 & 0 & Hybr. \\
\hline 05-02 & 118 & 1796 & 1800 & $\mathrm{H} 2$ & 100 & 1470 & 1 & 9.5 & 50 & 801.546 & 0 & Hybr. \\
\hline 05-02 & 118 & 1801 & 1827 & $\mathrm{H} 2$ & 100 & 1470 & 1 & 9.5 & 50 & 801.549 & $0 \quad 2$ & Hybr. \\
\hline 05-02 & 118 & 1828 & 1836 & $\mathrm{H} 2$ & 100 & 1470 & 1 & 9.5 & 20 & 801.605 & 3 & Hybr. \\
\hline 05-02 & 118 & 1837 & 1844 & $\mathrm{H} 2$ & 100 & 1470 & 1 & 9.5 & 10 & 801.636 & 3 & Hybr. \\
\hline 05-02 & 118 & 1845 & 1852 & $\mathrm{H} 2$ & 100 & 1470 & 1 & 9.5 & 5 & 801.655 & 3 & Hybr. \\
\hline 05-02 & 118 & 1854 & 1861 & $\mathrm{H} 2$ & 100 & 1470 & 1 & 9.5 & 25 & 801.667 & 3 & Hybr. \\
\hline 05-02 & 118 & 1863 & 1903 & $\mathrm{H} 2$ & 100 & 1470 & 1 & 9.5 & 30 & 801.663 & 3 & Hybr. \\
\hline 05-02 & 118 & 1904 & 1908 & $\mathrm{H} 2$ & 100 & 1470 & 1 & 9.5 & 40 & 801.646 & 3 & Hybr. \\
\hline 04-19 & 32 & 134 & 142 & $\mathrm{H} 2$ & 100 & 1520 & 0.1 & 7.5 & 25 & 801.3 & 0 & Circulator \\
\hline 04-19 & 32 & 143 & 152 & $\mathrm{H} 2$ & 100 & 1520 & 0.1 & 7.5 & 20 & 801.3 & 0 & Circulator \\
\hline 04-19 & 32 & 153 & 160 & $\mathrm{H} 2$ & 100 & 1520 & 0.1 & 7.5 & 10 & 801.3 & 0 & Circulator \\
\hline 04-19 & 32 & 162 & 170 & $\mathrm{H} 2$ & 100 & 1520 & 0.1 & 7.5 & 5 & 801.331 & 0 & Circulator \\
\hline 04-19 & 32 & 171 & 178 & $\mathrm{H} 2$ & 100 & 1520 & 1 & 7.5 & 5 & 801.35 & 0 & Circulator \\
\hline 04-19 & 32 & 180 & 188 & $\mathrm{H} 2$ & 100 & 1520 & 1 & 7.5 & 10 & 801.36 & 0 & Circulator \\
\hline 04-19 & 32 & 190 & 199 & $\mathrm{H} 2$ & 100 & 1520 & 1 & 7.5 & 20 & 801.366 & 0 & Circulator \\
\hline 04-19 & 32 & 201 & 209 & $\mathrm{H} 2$ & 100 & 1520 & 1 & 7.5 & 25 & 801.37 & 0 & Circulator \\
\hline 04-19 & 32 & 215 & 222 & $\mathrm{H} 2$ & 100 & 1520 & 0.2 & 7.5 & 25 & 801.374 & 0 & Circulator \\
\hline 04-19 & 32 & 223 & 230 & $\mathrm{H} 2$ & 100 & 1520 & 0.2 & 7.5 & 20 & 801.384 & 0 & Circulator \\
\hline 04-19 & 32 & 232 & 239 & $\mathrm{H} 2$ & 100 & 1520 & 0.2 & 7.5 & 10 & 801.398 & 0 & Circulator \\
\hline 04-19 & 32 & 241 & 248 & $\mathrm{H} 2$ & 100 & 1520 & 0.2 & 7.5 & 5 & 801.419 & 0 & Circulator \\
\hline $04-23$ & 44 & 17 & 25 & $\mathrm{H} 2$ & 100 & 1520 & 0.05 & 7.5 & 25 & 801.27 & 0 & Circulator \\
\hline $04-23$ & 44 & 26 & 33 & $\mathrm{H} 2$ & 100 & 1520 & 0.05 & 7.5 & 20 & 801.256 & 0 & Circulator \\
\hline $04-23$ & 44 & 34 & 49 & $\mathrm{H} 2$ & 100 & 1520 & 0.05 & 7.5 & 10 & 801.284 & 0 & Circulator \\
\hline $04-23$ & 44 & 50 & 60 & $\mathrm{H} 2$ & 100 & 1520 & 0.05 & 7.5 & 5 & 801.338 & 0 & Circulator \\
\hline 04-19 & 27 & & & $\mathrm{H} 2$ & 100 & & & 7.5 & & 803.664 & 0 & Circulator \\
\hline 04-19 & 28 & & & $\mathrm{H} 2$ & 100 & & & 7.5 & & 803.67 & 0 & Circulator \\
\hline $04-24$ & 72 & 749 & 758 & $\mathrm{H} 2+\mathrm{Air}$ & 0.04 & 300 & 1 & 9.5 & 18 & 808.321 & 0 & Circulator \\
\hline
\end{tabular}




\begin{tabular}{|c|c|c|c|c|c|c|c|c|c|c|c|c|}
\hline Date & Run & $\begin{array}{l}\text { Start } \\
\text { Pulse }\end{array}$ & $\begin{array}{l}\text { End } \\
\text { Pulse }\end{array}$ & $\begin{array}{l}\text { Gas } \\
\text { Species }\end{array}$ & $\begin{array}{l}\text { Gas } \\
\text { Conc. } \\
(\%)\end{array}$ & $\begin{array}{l}\text { Gas } \\
\text { Press. } \\
\text { (psi) }\end{array}$ & $\begin{array}{l}\text { Beam } \\
\text { Int. }\end{array}$ & $\begin{array}{l}\text { Beam } \\
\text { Length } \\
(\mu \mathrm{s})\end{array}$ & $\begin{array}{l}\text { Elec. } \\
\text { Field } \\
(\mathrm{MV} / \mathrm{m})\end{array}$ & $\begin{array}{l}\text { Freq. } \\
(\mathrm{MHz})\end{array}$ & $\begin{array}{l}\text { Mag. } \\
\text { Field } \\
(\mathrm{T})\end{array}$ & $\begin{array}{l}\text { Circulator } \\
\text { /Hybr. }\end{array}$ \\
\hline $04-24$ & 72 & 759 & 768 & $\mathrm{H} 2+\mathrm{Air}$ & 0.04 & 300 & 1 & 9.5 & 10 & 808.252 & 0 & Circulator \\
\hline $04-24$ & 72 & 769 & 778 & $\mathrm{H} 2+\mathrm{Air}$ & 0.04 & 300 & 1 & 9.5 & 5 & 808.206 & 0 & Circulator \\
\hline 05-02 & 123 & 1955 & 1959 & $\mathrm{H} 2+\mathrm{Air}$ & 0.04 & 300 & 1 & 9.5 & 18 & 808.455 & 3 & Hybr. \\
\hline 05-02 & 123 & 1960 & 1964 & $\mathrm{H} 2+\mathrm{Air}$ & 0.04 & 300 & 1 & 9.5 & 10 & 808.449 & 3 & Hybr. \\
\hline 05-03 & 132 & 2875 & 2883 & $\mathrm{H} 2+\mathrm{Air}$ & 0.04 & 300 & 0.2 & 9.5 & 10 & 808.441 & 0 & Hybr. \\
\hline 05-03 & 133 & 2884 & 2885 & $\mathrm{H} 2+\mathrm{Air}$ & 0.04 & 300 & 0.2 & 9.5 & 10 & 808.441 & 0 & Hybr. \\
\hline 05-03 & 133 & 2886 & 2895 & $\mathrm{H} 2+\mathrm{Air}$ & 0.04 & 300 & 0.2 & 9.5 & 18 & 808.444 & 0 & Hybr. \\
\hline $04-24$ & 70 & 672 & 684 & $\mathrm{H} 2+\mathrm{Air}$ & 0.2 & 300 & 1 & 9.5 & 18 & 808.316 & 0 & Circulator \\
\hline $04-24$ & 70 & 686 & 696 & $\mathrm{H} 2+\mathrm{Air}$ & 0.2 & 300 & 1 & 9.5 & 10 & 808.22 & 0 & Circulator \\
\hline $04-24$ & 70 & 698 & 708 & $\mathrm{H} 2+\mathrm{Air}$ & 0.2 & 300 & 1 & 9.5 & 5 & 808.188 & 0 & Circulator \\
\hline 05-02 & 121 & 1933 & 1937 & $\mathrm{H} 2+\mathrm{Air}$ & 0.2 & 300 & 1 & 9.5 & 18 & 808.399 & 3 & Hybr. \\
\hline 05-02 & 121 & 1939 & 1943 & $\mathrm{H} 2+\mathrm{Air}$ & 0.2 & 300 & 1 & 9.5 & 10 & 808.393 & 3 & Hybr. \\
\hline 05-03 & 143 & 3127 & 3136 & $\mathrm{H} 2+\mathrm{Air}$ & 0.2 & 300 & 0.2 & 9.5 & 10 & 808.433 & 0 & Hybr. \\
\hline 05-03 & 143 & 3137 & 3149 & $\mathrm{H} 2+\mathrm{Air}$ & 0.2 & 300 & 0.2 & 9.5 & 18 & 808.422 & 0 & Hybr. \\
\hline $04-24$ & 68 & 597 & 607 & $\mathrm{H} 2+\mathrm{Air}$ & 1 & 300 & 1 & 9.5 & 18 & 808.461 & 0 & Circulator \\
\hline $04-24$ & 68 & 608 & 617 & $\mathrm{H} 2+\mathrm{Air}$ & 1 & 300 & 1 & 9.5 & 10 & 808.448 & 0 & Circulator \\
\hline $04-24$ & 68 & 618 & 627 & $\mathrm{H} 2+\mathrm{Air}$ & 1 & 300 & 1 & 9.5 & 5 & 808.452 & 0 & Circulator \\
\hline 05-03 & 137 & 2994 & 3003 & $\mathrm{H} 2+\mathrm{Air}$ & 1 & 300 & 0.2 & 9.5 & 10 & 808.398 & 0 & Hybr. \\
\hline 05-03 & 137 & 3004 & 3013 & $\mathrm{H} 2+\mathrm{Air}$ & 1 & 300 & 0.2 & 9.5 & 18 & 808.396 & 0 & Hybr. \\
\hline $04-24$ & 65 & 478 & 488 & $\mathrm{H} 2+\mathrm{Air}$ & 5 & 300 & 1 & 9.5 & 5 & 808.34 & 0 & Circulator \\
\hline $04-24$ & 65 & 490 & 499 & $\mathrm{H} 2+\mathrm{Air}$ & 5 & 300 & 1 & 9.5 & 10 & 808.34 & 0 & Circulator \\
\hline $04-24$ & 65 & 500 & 512 & $\mathrm{H} 2+\mathrm{Air}$ & 5 & 300 & 1 & 9.5 & 18 & 808.335 & 0 & Circulator \\
\hline $04-24$ & 58 & 307 & 321 & $\mathrm{H} 2+\mathrm{Air}$ & 10 & 735 & 1 & 9.5 & 25 & 805.037 & 0 & Circulator \\
\hline $04-24$ & 58 & 322 & 331 & $\mathrm{H} 2+\mathrm{Air}$ & 10 & 735 & 1 & 9.5 & 20 & 805.034 & 0 & Circulator \\
\hline $04-24$ & 58 & 332 & 340 & $\mathrm{H} 2+\mathrm{Air}$ & 10 & 735 & 1 & 9.5 & 10 & 805.06 & 0 & Circulator \\
\hline $04-24$ & 58 & 341 & 350 & $\mathrm{H} 2+\mathrm{Air}$ & 10 & 735 & 1 & 9.5 & 5 & 805.08 & 0 & Circulator \\
\hline 05-03 & 131 & 2843 & 2852 & $\mathrm{H} 2+\mathrm{Air}$ & 0.04 & 800 & 0.2 & 9.5 & 10 & 805.452 & 0 & Hybr. \\
\hline 05-03 & 131 & 2854 & 2863 & $\mathrm{H} 2+\mathrm{Air}$ & 0.04 & 800 & 0.2 & 9.5 & 25 & 805.448 & 0 & Hybr. \\
\hline 05-03 & 131 & 2864 & 2874 & $\mathrm{H} 2+\mathrm{Air}$ & 0.04 & 800 & 0.2 & 9.5 & 40 & 805.419 & 0 & Hybr. \\
\hline 05-03 & 141 & 3077 & 3086 & $\mathrm{H} 2+\mathrm{Air}$ & 0.2 & 800 & 0.2 & 9.5 & 10 & 805.432 & 0 & Hybr. \\
\hline 05-03 & 141 & 3088 & 3099 & $\mathrm{H} 2+\mathrm{Air}$ & 0.2 & 800 & 0.2 & 9.5 & 20 & 805.43 & 0 & Hybr. \\
\hline 05-03 & 142 & 3116 & 3125 & $\mathrm{H} 2+\mathrm{Air}$ & 0.2 & 800 & 0.2 & 9.5 & 25 & 805.421 & 0 & Hybr. \\
\hline 05-03 & 136 & 2964 & 2973 & $\mathrm{H} 2+\mathrm{Air}$ & 1 & 800 & 0.2 & 9.5 & 10 & 805.378 & 0 & Hybr. \\
\hline 05-03 & 136 & 2974 & 2983 & $\mathrm{H} 2+\mathrm{Air}$ & 1 & 800 & 0.2 & 9.5 & 25 & 805.376 & 0 & Hybr. \\
\hline 05-03 & 136 & 2984 & 2993 & $\mathrm{H} 2+\mathrm{Air}$ & 1 & 800 & 0.2 & 9.5 & 40 & 805.341 & 0 & Hybr. \\
\hline 05-03 & 129 & 2316 & 2328 & $\mathrm{H} 2+\mathrm{Air}$ & 0.04 & 1100 & 1 & 9.5 & 40 & 803.711 & 3 & Hybr. \\
\hline 05-03 & 129 & 2329 & 2339 & $\mathrm{H} 2+\mathrm{Air}$ & 0.04 & 1100 & 1 & 9.5 & 25 & 803.594 & 3 & Hybr. \\
\hline 05-03 & 129 & 2341 & 2350 & $\mathrm{H} 2+\mathrm{Air}$ & 0.04 & 1100 & 1 & 9.5 & 10 & 803.722 & 3 & Hybr. \\
\hline 05-03 & 129 & 2351 & 2351 & $\mathrm{H} 2+\mathrm{Air}$ & 0.04 & 1100 & 1 & 9.5 & 10 & 803.749 & 2.96 & Hybr. \\
\hline 05-03 & 129 & 2352 & 2352 & $\mathrm{H} 2+\mathrm{Air}$ & 0.04 & 1100 & 1 & 9.5 & 10 & 803.753 & 2.92 & Hybr. \\
\hline
\end{tabular}




\begin{tabular}{|c|c|c|c|c|c|c|c|c|c|c|c|c|}
\hline Date & Run & $\begin{array}{l}\text { Start } \\
\text { Pulse }\end{array}$ & $\begin{array}{l}\text { End } \\
\text { Pulse }\end{array}$ & $\begin{array}{l}\text { Gas } \\
\text { Species }\end{array}$ & $\begin{array}{l}\text { Gas } \\
\text { Conc. } \\
(\%)\end{array}$ & $\begin{array}{l}\text { Gas } \\
\text { Press. } \\
\text { (psi) }\end{array}$ & $\begin{array}{l}\text { Beam } \\
\text { Int. }\end{array}$ & $\begin{array}{l}\text { Beam } \\
\text { Length } \\
(\mu \mathrm{s})\end{array}$ & $\begin{array}{l}\text { Elec. } \\
\text { Field } \\
(\mathrm{MV} / \mathrm{m})\end{array}$ & $\begin{array}{l}\text { Freq. } \\
(\mathrm{MHz})\end{array}$ & $\begin{array}{l}\text { Mag. } \\
\text { Field } \\
(\mathrm{T})\end{array}$ & $\begin{array}{l}\text { Circulator } \\
\text { /Hybr. }\end{array}$ \\
\hline 05-03 & 129 & 2353 & 2397 & $\mathrm{H} 2+\mathrm{Air}$ & 0.04 & 1100 & 1 & 9.5 & 10 & 803.754 & $\begin{array}{l}2.88 \\
2.69\end{array}$ & Hybr. \\
\hline 05-03 & 129 & 2398 & 2401 & H2+Air & 0.04 & 1100 & 1 & 9.5 & 10 & 803.757 & $\begin{array}{l}2.65 \\
2.54\end{array}$ & Hybr. \\
\hline 05-03 & 129 & 2402 & 2409 & $\mathrm{H} 2+\mathrm{Air}$ & 0.04 & 1100 & 1 & 9.5 & 10 & 803.761 & $\begin{array}{l}2.5 \\
2.2\end{array}$ & Hybr. \\
\hline 05-03 & 129 & 2410 & 2414 & $\mathrm{H} 2+\mathrm{Air}$ & 0.04 & 1100 & 1 & 9.5 & 10 & 803.764 & $\begin{array}{l}2.17 \\
2.03\end{array}$ & Hybr. \\
\hline 05-03 & 129 & 2415 & 2417 & $\mathrm{H} 2+\mathrm{Air}$ & 0.04 & 1100 & 1 & 9.5 & 10 & 803.768 & $\begin{array}{l}2.01 \\
1.94\end{array}$ & Hybr. \\
\hline 05-03 & 129 & 2418 & 2423 & $\mathrm{H} 2+\mathrm{Air}$ & 0.04 & 1100 & 1 & 9.5 & 10 & 803.769 & $\begin{array}{l}1.92 \\
1.77\end{array}$ & Hybr. \\
\hline 05-03 & 129 & 2424 & 2425 & $\mathrm{H} 2+\mathrm{Air}$ & 0.04 & 1100 & 1 & 9.5 & 10 & 803.773 & $\begin{array}{l}1.74 \\
1.65\end{array}$ & Hybr. \\
\hline 05-03 & 129 & 2495 & 2683 & $\mathrm{H} 2+\mathrm{Air}$ & 0.04 & 1100 & 1 & 9.5 & 10 & 803.775 & $\begin{array}{l}1.59 \\
1.48\end{array}$ & Hybr. \\
\hline 05-03 & 129 & 2684 & 2693 & $\mathrm{H} 2+\mathrm{Air}$ & 0.04 & 1100 & 1 & 9.5 & 10 & 803.778 & $\begin{array}{l}1.46 \\
1.17\end{array}$ & Hybr. \\
\hline 05-03 & 129 & 2694 & 2706 & $\mathrm{H} 2+\mathrm{Air}$ & 0.04 & 1100 & 1 & 9.5 & 10 & 803.784 & $\begin{array}{l}1.15 \\
0.85\end{array}$ & Hybr. \\
\hline 05-03 & 129 & 2707 & 2726 & $\mathrm{H} 2+\mathrm{Air}$ & 0.04 & 1100 & 1 & 9.5 & 10 & 803.791 & $\begin{array}{l}0.83 \\
0.39\end{array}$ & Hybr. \\
\hline 05-03 & 129 & 2727 & 2733 & $\mathrm{H} 2+\mathrm{Air}$ & 0.04 & 1100 & 1 & 9.5 & 10 & 803.803 & $\begin{array}{l}0.37 \\
0.26\end{array}$ & Hybr. \\
\hline 05-03 & 129 & 2734 & 2752 & $\mathrm{H} 2+\mathrm{Air}$ & 0.04 & 1100 & 1 & 9.5 & 10 & 803.806 & $\begin{array}{l}0.25 \\
0\end{array}$ & Hybr. \\
\hline 05-03 & 130 & 2754 & 2763 & $\mathrm{H} 2+\mathrm{Air}$ & 0.04 & 1100 & 0.2 & 9.5 & 10 & 803.821 & 0 & Hybr. \\
\hline 05-03 & 130 & 2765 & 2831 & $\mathrm{H} 2+\mathrm{Air}$ & 0.04 & 1100 & 0.2 & 9.5 & 25 & 803.825 & 0 & Hybr. \\
\hline 05-03 & 130 & 2833 & 2842 & $\mathrm{H} 2+\mathrm{Air}$ & 0.04 & 1100 & 0.2 & 9.5 & 40 & 803.804 & 0 & Hybr. \\
\hline 05-03 & 128 & 2284 & 2293 & $\mathrm{H} 2+\mathrm{Air}$ & 0.2 & 1100 & 1 & 9.5 & 10 & 803.687 & 3 & Hybr. \\
\hline 05-03 & 128 & 2296 & 2305 & $\mathrm{H} 2+\mathrm{Air}$ & 0.2 & 1100 & 1 & 9.5 & 25 & 803.687 & 3 & Hybr. \\
\hline 05-03 & 128 & 2306 & 2315 & $\mathrm{H} 2+\mathrm{Air}$ & 0.2 & 1100 & 1 & 9.5 & 40 & 803.656 & 3 & Hybr. \\
\hline 05-03 & 139 & 3043 & 3052 & $\mathrm{H} 2+\mathrm{Air}$ & 0.2 & 1100 & 0.2 & 9.5 & 10 & 803.697 & 0 & Hybr. \\
\hline 05-03 & 139 & 3053 & 3064 & $\mathrm{H} 2+\mathrm{Air}$ & 0.2 & 1100 & 0.2 & 9.5 & 25 & 803.698 & 0 & Hybr. \\
\hline 05-03 & 139 & 3065 & 3076 & $\mathrm{H} 2+\mathrm{Air}$ & 0.2 & 1100 & 0.2 & 9.5 & 40 & 803.68 & 0 & Hybr. \\
\hline $04-24$ & 67 & 555 & 565 & $\mathrm{H} 2+\mathrm{Air}$ & 1 & 1100 & 1 & 9.5 & 25 & 803.473 & 0 & Circulator \\
\hline $04-24$ & 67 & 566 & 575 & $\mathrm{H} 2+\mathrm{Air}$ & 1 & 1100 & 1 & 9.5 & 20 & 803.474 & 0 & Circulator \\
\hline $04-24$ & 67 & 576 & 585 & $\mathrm{H} 2+\mathrm{Air}$ & 1 & 1100 & 1 & 9.5 & 10 & 803.486 & 0 & Circulator \\
\hline $04-24$ & 67 & 586 & 596 & $\mathrm{H} 2+\mathrm{Air}$ & 1 & 1100 & 1 & 9.5 & 5 & 803.487 & 0 & Circulator \\
\hline 05-03 & 127 & 2255 & 2264 & $\mathrm{H} 2+\mathrm{Air}$ & 1 & 1100 & 1 & 9.5 & 10 & 803.525 & 3 & Hybr. \\
\hline 05-03 & 127 & 2265 & 2273 & $\mathrm{H} 2+\mathrm{Air}$ & 1 & 1100 & 1 & 9.5 & 25 & 803.484 & 3 & Hybr. \\
\hline 05-03 & 127 & 2274 & 2283 & $\mathrm{H} 2+\mathrm{Air}$ & 1 & 1100 & 1 & 9.5 & 40 & 803.421 & 3 & Hybr. \\
\hline 05-03 & 135 & 2934 & 2943 & $\mathrm{H} 2+\mathrm{Air}$ & 1 & 1100 & 0.2 & 9.5 & 10 & 803.614 & 0 & Hybr. \\
\hline
\end{tabular}




\begin{tabular}{|c|c|c|c|c|c|c|c|c|c|c|c|c|}
\hline Date & Run & $\begin{array}{l}\text { Start } \\
\text { Pulse }\end{array}$ & $\begin{array}{l}\text { End } \\
\text { Pulse }\end{array}$ & $\begin{array}{l}\text { Gas } \\
\text { Species }\end{array}$ & $\begin{array}{l}\text { Gas } \\
\text { Conc. } \\
(\%)\end{array}$ & $\begin{array}{l}\text { Gas } \\
\text { Press. } \\
\text { (psi) }\end{array}$ & $\begin{array}{l}\text { Beam } \\
\text { Int. }\end{array}$ & $\begin{array}{l}\text { Beam } \\
\text { Length } \\
(\mu \mathrm{s})\end{array}$ & $\begin{array}{l}\text { Elec. } \\
\text { Field } \\
(\mathrm{MV} / \mathrm{m})\end{array}$ & $\begin{array}{l}\text { Freq. } \\
(\mathrm{MHz})\end{array}$ & $\begin{array}{l}\text { Mag. } \\
\text { Field } \\
(\mathrm{T})\end{array}$ & $\begin{array}{l}\text { Circulator } \\
\text { /Hybr. }\end{array}$ \\
\hline 05-03 & 135 & 2944 & 2953 & $\mathrm{H} 2+\mathrm{Air}$ & 1 & 1100 & 0.2 & 9.5 & 25 & 803.615 & 0 & Hybr. \\
\hline 05-03 & 135 & 2954 & 2963 & $\mathrm{H} 2+\mathrm{Air}$ & 1 & 1100 & 0.2 & 9.5 & 40 & 803.595 & 0 & Hybr. \\
\hline $04-24$ & 62 & 436 & 445 & $\mathrm{H} 2+\mathrm{Air}$ & 5 & 1100 & 1 & 9.5 & 25 & 803.006 & 0 & Circulator \\
\hline $04-24$ & 62 & 446 & 457 & $\mathrm{H} 2+\mathrm{Air}$ & 5 & 1100 & 1 & 9.5 & 20 & 803.007 & 0 & Circulator \\
\hline $04-24$ & 62 & 458 & 466 & $\mathrm{H} 2+\mathrm{Air}$ & 5 & 1100 & 1 & 9.5 & 10 & 803.019 & 0 & Circulator \\
\hline $04-24$ & 62 & 467 & 476 & $\mathrm{H} 2+\mathrm{Air}$ & 5 & 1100 & 1 & 9.5 & 5 & 803.038 & 0 & Circulator \\
\hline $04-24$ & 59 & 351 & 361 & $\mathrm{H} 2+\mathrm{Air}$ & 6.78 & 1100 & 1 & 9.5 & 25 & 802.921 & 0 & Circulator \\
\hline $04-24$ & 59 & 362 & 372 & $\mathrm{H} 2+\mathrm{Air}$ & 6.78 & 1100 & 1 & 9.5 & 20 & 802.924 & 0 & Circulator \\
\hline $04-24$ & 59 & 373 & 382 & $\mathrm{H} 2+\mathrm{Air}$ & 6.78 & 1100 & 1 & 9.5 & 10 & 802.947 & 0 & Circulator \\
\hline $04-24$ & 59 & 383 & 392 & $\mathrm{H} 2+\mathrm{Air}$ & 6.78 & 1100 & 1 & 9.5 & 5 & 802.971 & 0 & Circulator \\
\hline 05-03 & 144 & 3150 & 3159 & $\mathrm{H} 2+\mathrm{Air}$ & 0.022 & 1385 & 0.2 & 9.5 & 10 & 802.158 & 0 & Hybr. \\
\hline 05-03 & 144 & 3160 & 3170 & $\mathrm{H} 2+\mathrm{Air}$ & 0.022 & 1385 & 0.2 & 9.5 & 25 & 802.163 & 0 & Hybr. \\
\hline 05-03 & 144 & 3172 & 3182 & $\mathrm{H} 2+\mathrm{Air}$ & 0.022 & 1385 & 0.2 & 9.5 & 40 & 802.147 & 0 & Hybr. \\
\hline 05-03 & 144 & 3183 & 3192 & $\mathrm{H} 2+\mathrm{Air}$ & 0.022 & 1385 & 0.2 & 9.5 & 50 & 802.128 & 0 & Hybr. \\
\hline $04-24$ & 71 & 709 & 718 & $\mathrm{H} 2+\mathrm{Air}$ & 0.04 & 1450 & 1 & 9.5 & 25 & 801.678 & 0 & Circulator \\
\hline $04-24$ & 71 & 719 & 728 & $\mathrm{H} 2+\mathrm{Air}$ & 0.04 & 1450 & 1 & 9.5 & 20 & 801.665 & 0 & Circulator \\
\hline $04-24$ & 71 & 729 & 738 & $\mathrm{H} 2+\mathrm{Air}$ & 0.04 & 1450 & 1 & 9.5 & 10 & 801.683 & 0 & Circulator \\
\hline $04-24$ & 71 & 739 & 748 & $\mathrm{H} 2+\mathrm{Air}$ & 0.04 & 1450 & 1 & 9.5 & 5 & 801.703 & 0 & Circulator \\
\hline 04-24 & 69 & 628 & 640 & $\mathrm{H} 2+\mathrm{Air}$ & 0.2 & 1450 & 1 & 9.5 & 25 & 801.642 & 0 & Circulator \\
\hline $04-24$ & 69 & 641 & 650 & $\mathrm{H} 2+\mathrm{Air}$ & 0.2 & 1450 & 1 & 9.5 & 20 & 801.637 & 0 & Circulator \\
\hline $04-24$ & 69 & 651 & 659 & $\mathrm{H} 2+\mathrm{Air}$ & 0.2 & 1450 & 1 & 9.5 & 10 & 801.65 & 0 & Circulator \\
\hline $04-24$ & 69 & 660 & 670 & $\mathrm{H} 2+\mathrm{Air}$ & 0.2 & 1450 & 1 & 9.5 & 5 & 801.773 & 0 & Circulator \\
\hline $04-24$ & 61 & 393 & 403 & $\mathrm{H} 2+\mathrm{Air}$ & 5 & 1450 & 1 & 9.5 & 25 & 800.884 & 0 & Circulator \\
\hline $04-24$ & 61 & 404 & 413 & $\mathrm{H} 2+\mathrm{Air}$ & 5 & 1450 & 1 & 9.5 & 20 & 800.894 & 0 & Circulator \\
\hline $04-24$ & 61 & 414 & 424 & $\mathrm{H} 2+\mathrm{Air}$ & 5 & 1450 & 1 & 9.5 & 10 & 800.911 & 0 & Circulator \\
\hline $04-24$ & 61 & 425 & 435 & $\mathrm{H} 2+\mathrm{Air}$ & 5 & 1450 & 1 & 9.5 & 5 & 800.93 & 0 & Circulator \\
\hline $04-27$ & 102 & 1603 & 1612 & $\mathrm{H} 2+\mathrm{Air}$ & 0.001 & 1470 & 1 & 9.5 & 25 & 801.606 & 0 & Circulator \\
\hline $04-27$ & 102 & 1613 & 1622 & $\mathrm{H} 2+\mathrm{Air}$ & 0.001 & 1470 & 1 & 9.5 & 20 & 801.615 & 0 & Circulator \\
\hline $04-27$ & 102 & 1752 & 1762 & $\mathrm{H} 2+\mathrm{Air}$ & 0.001 & 1470 & 1 & 9.5 & 10 & 801.637 & 0 & Circulator \\
\hline $04-27$ & 102 & 1763 & 1772 & $\mathrm{H} 2+\mathrm{Air}$ & 0.001 & 1470 & 1 & 9.5 & 5 & 801.662 & 0 & Circulator \\
\hline $04-27$ & 99 & 1562 & 1572 & $\mathrm{H} 2+\mathrm{Air}$ & 0.002 & 1470 & 1 & 9.5 & 25 & 801.612 & 0 & Circulator \\
\hline $04-27$ & 99 & 1573 & 1582 & $\mathrm{H} 2+\mathrm{Air}$ & 0.002 & 1470 & 1 & 9.5 & 20 & 801.621 & 0 & Circulator \\
\hline $04-27$ & 99 & 1583 & 1592 & $\mathrm{H} 2+\mathrm{Air}$ & 0.002 & 1470 & 1 & 9.5 & 10 & 801.642 & 0 & Circulator \\
\hline $04-27$ & 101 & 1593 & 1602 & $\mathrm{H} 2+\mathrm{Air}$ & 0.002 & 1470 & 1 & 9.5 & 5 & 801.667 & 0 & Circulator \\
\hline $04-27$ & 98 & 1553 & 1562 & $\mathrm{H} 2+\mathrm{Air}$ & 0.01 & 1470 & 1 & 9.5 & 25 & 801.608 & 0 & Circulator \\
\hline $04-27$ & 97 & 1543 & 1552 & $\mathrm{H} 2+\mathrm{Air}$ & 0.025 & 1470 & 1 & 9.5 & 25 & 801.596 & 0 & Circulator \\
\hline 05-02 & 122 & 1945 & 1949 & $\mathrm{H} 2+\mathrm{Air}$ & 0.04 & 1470 & 1 & 9.5 & 25 & 801.691 & 3 & Hybr. \\
\hline 05-02 & 122 & 1950 & 1954 & $\mathrm{H} 2+\mathrm{Air}$ & 0.04 & 1470 & 1 & 9.5 & 50 & 801.667 & 3 & Hybr. \\
\hline $04-27$ & 95 & 1563 & 1570 & $\mathrm{H} 2+\mathrm{Air}$ & 0.05 & 1470 & 1 & 9.5 & 25 & 801.596 & 0 & Circulator \\
\hline 05-02 & 120 & 1921 & 1925 & $\mathrm{H} 2+\mathrm{Air}$ & 0.2 & 1470 & 1 & 9.5 & 25 & 801.706 & 3 & Hybr. \\
\hline
\end{tabular}




\begin{tabular}{|c|c|c|c|c|c|c|c|c|c|c|c|c|}
\hline Date & Run & $\begin{array}{l}\text { Start } \\
\text { Pulse }\end{array}$ & $\begin{array}{l}\text { End } \\
\text { Pulse }\end{array}$ & $\begin{array}{l}\text { Gas } \\
\text { Species }\end{array}$ & $\begin{array}{l}\text { Gas } \\
\text { Conc. } \\
(\%)\end{array}$ & $\begin{array}{l}\text { Gas } \\
\text { Press. } \\
\text { (psi) }\end{array}$ & $\begin{array}{l}\text { Beam } \\
\text { Int. }\end{array}$ & $\begin{array}{l}\text { Beam } \\
\text { Length } \\
(\mu \mathrm{s})\end{array}$ & $\begin{array}{l}\text { Elec. } \\
\text { Field } \\
(\mathrm{MV} / \mathrm{m})\end{array}$ & $\begin{array}{l}\text { Freq. } \\
(\mathrm{MHz})\end{array}$ & $\begin{array}{l}\text { Mag. } \\
\text { Field } \\
(\mathrm{T})\end{array}$ & $\begin{array}{l}\text { Circulator } \\
\text { /Hybr. }\end{array}$ \\
\hline 05-02 & 120 & 1926 & 1931 & $\mathrm{H} 2+\mathrm{Air}$ & 0.2 & 1470 & 1 & 9.5 & 50 & 801.666 & 3 & Hybr. \\
\hline 05-03 & 138 & 3014 & 3022 & $\mathrm{H} 2+\mathrm{Air}$ & 0.2 & 1470 & 0.2 & 9.5 & 10 & 801.672 & 0 & Hybr. \\
\hline 05-03 & 138 & 3023 & 3032 & $\mathrm{H} 2+\mathrm{Air}$ & 0.2 & 1470 & 0.2 & 9.5 & 25 & 801.683 & 0 & Hybr. \\
\hline 05-03 & 138 & 3033 & 3042 & $\mathrm{H} 2+\mathrm{Air}$ & 0.2 & 1470 & 0.2 & 9.5 & 40 & 801.664 & 0 & Hybr. \\
\hline 04-24 & 66 & 514 & 524 & $\mathrm{H} 2+\mathrm{Air}$ & 1 & 1470 & 1 & 9.5 & 25 & 801.46 & 0 & Circulator \\
\hline $04-24$ & 66 & 525 & 534 & $\mathrm{H} 2+\mathrm{Air}$ & 1 & 1470 & 1 & 9.5 & 20 & 801.45 & 0 & Circulator \\
\hline 04-24 & 66 & 535 & 544 & $\mathrm{H} 2+\mathrm{Air}$ & 1 & 1470 & 1 & 9.5 & 10 & 801.457 & 0 & Circulator \\
\hline 04-24 & 66 & 545 & 554 & $\mathrm{H} 2+\mathrm{Air}$ & 1 & 1470 & 1 & 9.5 & 5 & 801.471 & 0 & Circulator \\
\hline 05-02 & 119 & 1909 & 1915 & $\mathrm{H} 2+\mathrm{Air}$ & 1 & 1470 & 1 & 9.5 & 50 & 801.476 & 3 & Hybr. \\
\hline 05-02 & 119 & 1916 & 1920 & $\mathrm{H} 2+\mathrm{Air}$ & 1 & 1470 & 1 & 9.5 & 25 & 801.447 & 3 & Hybr. \\
\hline 05-03 & 134 & 2896 & 2907 & $\mathrm{H} 2+\mathrm{Air}$ & 1 & 1470 & 0.2 & 9.5 & 10 & 801.623 & 0 & Hybr. \\
\hline 05-03 & 134 & 2914 & 2923 & $\mathrm{H} 2+\mathrm{Air}$ & 1 & 1470 & 0.2 & 9.5 & 25 & 801.623 & 0 & Hybr. \\
\hline 05-03 & 134 & 2924 & 2933 & $\mathrm{H} 2+\mathrm{Air}$ & 1 & 1470 & 0.2 & 9.5 & 40 & 801.619 & 0 & Hybr. \\
\hline 05-03 & 126 & 2193 & 2200 & $\mathrm{H} 2+\mathrm{N} 2$ & 1 & 1100 & 1 & 9.5 & 10 & 803.633 & 0 & Hybr. \\
\hline \multirow[t]{2}{*}{ 05-03 } & 126 & 2201 & 2208 & $\mathrm{H} 2+\mathrm{N} 2$ & 1 & 1100 & 1 & 9.5 & 10 & 803.633 & 0.11 & Hybr. \\
\hline & & & & & & & & & & & 0.59 & \\
\hline \multirow[t]{2}{*}{ 05-03 } & 126 & 2209 & 2213 & $\mathrm{H} 2+\mathrm{N} 2$ & 1 & 1100 & 1 & 9.5 & 10 & 803.644 & 0.67 & Hybr. \\
\hline & & & & & & & & & & & 1 & \\
\hline \multirow[t]{2}{*}{ 05-03 } & 126 & 2214 & 2223 & $\mathrm{H} 2+\mathrm{N} 2$ & 1 & 1100 & 1 & 9.5 & 10 & 803.648 & 1.07 & Hybr. \\
\hline & & & & & & & & & & & 1.59 & \\
\hline \multirow[t]{2}{*}{ 05-03 } & 126 & 2224 & 2227 & $\mathrm{H} 2+\mathrm{N} 2$ & 1 & 1100 & 1 & 9.5 & 10 & 803.653 & 1.63 & Hybr. \\
\hline & & & & & & & & & & & 1.85 & \\
\hline \multirow[t]{2}{*}{$05-03$} & 126 & 2228 & 2235 & $\mathrm{H} 2+\mathrm{N} 2$ & 1 & 1100 & 1 & 9.5 & 10 & 803.655 & 1.91 & Hybr. \\
\hline & & & & & & & & & & & 2.24 & \\
\hline \multirow[t]{2}{*}{ 05-03 } & 126 & 2236 & 2241 & $\mathrm{H} 2+\mathrm{N} 2$ & 1 & 1100 & 1 & 9.5 & 10 & 803.659 & 2.59 & Hybr. \\
\hline & & & & & & & & & & & 2.80 & \\
\hline \multirow[t]{2}{*}{$05-03$} & 126 & 2242 & 2244 & $\mathrm{H} 2+\mathrm{N} 2$ & 1 & 1100 & 1 & 9.5 & 10 & 803.663 & 2.87 & Hybr. \\
\hline & & & & & & & & & & & 2.96 & \\
\hline 05-03 & 126 & 2245 & 2254 & $\mathrm{H} 2+\mathrm{N} 2$ & 1 & 1100 & 1 & 9.5 & 10 & 803.648 & 3 & Hybr. \\
\hline $04-25$ & 87 & 1502 & 1511 & $\mathrm{H} 2+\mathrm{SF} 6$ & 0.00004 & 300 & 1 & 9.5 & 18 & 808.439 & 0 & Circulator \\
\hline $04-25$ & 87 & 1512 & 1519 & $\mathrm{H} 2+\mathrm{SF} 6$ & 0.00004 & 300 & 1 & 9.5 & 10 & 808.448 & 0 & Circulator \\
\hline $04-25$ & 87 & 1520 & 1529 & $\mathrm{H} 2+\mathrm{SF} 6$ & 0.00004 & 300 & 1 & 9.5 & 5 & 808.448 & 0 & Circulator \\
\hline $04-25$ & 84 & 1188 & 1321 & $\mathrm{H} 2+\mathrm{SF} 6$ & 0.0002 & 300 & 1 & 9.5 & 18 & 808.467 & 0 & Circulator \\
\hline $04-25$ & 84 & 1322 & 1406 & $\mathrm{H} 2+\mathrm{SF} 6$ & 0.0002 & 300 & 1 & 9.5 & 10 & 808.472 & 0 & Circulator \\
\hline $04-25$ & 84 & 1407 & 1416 & $\mathrm{H} 2+\mathrm{SF} 6$ & 0.0002 & 300 & 1 & 9.5 & 5 & 808.472 & 0 & Circulator \\
\hline $04-25$ & 81 & 1045 & 1055 & $\mathrm{H} 2+\mathrm{SF} 6$ & 0.002 & 300 & 1 & 9.5 & 18 & 808.461 & 0 & Circulator \\
\hline $04-25$ & 81 & 1056 & 1066 & $\mathrm{H} 2+\mathrm{SF} 6$ & 0.002 & 300 & 1 & 9.5 & 10 & 808.449 & 0 & Circulator \\
\hline $04-25$ & 81 & 1067 & 1076 & $\mathrm{H} 2+\mathrm{SF} 6$ & 0.002 & 300 & 1 & 9.5 & 5 & 808.458 & 0 & Circulator \\
\hline $04-25$ & 78 & 931 & 941 & $\mathrm{H} 2+\mathrm{SF} 6$ & 0.01 & 300 & 1 & 9.5 & 18 & 808.441 & 0 & Circulator \\
\hline $04-25$ & 78 & 942 & 950 & $\mathrm{H} 2+\mathrm{SF} 6$ & 0.01 & 300 & 1 & 9.5 & 10 & 808.433 & 0 & Circulator \\
\hline $04-25$ & 78 & 951 & 960 & $\mathrm{H} 2+\mathrm{SF} 6$ & 0.01 & 300 & 1 & 9.5 & 5 & 808.443 & 0 & Circulator \\
\hline $04-25$ & 73 & 779 & 787 & $\mathrm{H} 2+\mathrm{SF} 6$ & 0.5 & 300 & 1 & 9.5 & 5 & 806.53 & 0 & Circulator \\
\hline
\end{tabular}




\begin{tabular}{|c|c|c|c|c|c|c|c|c|c|c|c|c|}
\hline Date & Run & $\begin{array}{l}\text { Start } \\
\text { Pulse }\end{array}$ & $\begin{array}{l}\text { End } \\
\text { Pulse }\end{array}$ & $\begin{array}{l}\text { Gas } \\
\text { Species }\end{array}$ & $\begin{array}{l}\text { Gas } \\
\text { Conc. } \\
(\%)\end{array}$ & $\begin{array}{l}\text { Gas } \\
\text { Press. } \\
\text { (psi) }\end{array}$ & $\begin{array}{l}\text { Beam } \\
\text { Int. }\end{array}$ & $\begin{array}{l}\text { Beam } \\
\text { Length } \\
(\mu \mathrm{s})\end{array}$ & $\begin{array}{l}\text { Elec. } \\
\text { Field } \\
(\mathrm{MV} / \mathrm{m})\end{array}$ & $\begin{array}{l}\text { Freq. } \\
(\mathrm{MHz})\end{array}$ & $\begin{array}{l}\text { Mag. } \\
\text { Field } \\
(\mathrm{T})\end{array}$ & $\begin{array}{l}\text { Circulator } \\
\text { /Hybr. }\end{array}$ \\
\hline $04-25$ & 73 & 788 & 796 & $\mathrm{H} 2+\mathrm{SF} 6$ & 0.5 & 300 & 1 & 9.5 & 10 & 806.551 & 0 & Circulator \\
\hline $04-25$ & 73 & 797 & 805 & $\mathrm{H} 2+\mathrm{SF} 6$ & 0.5 & 300 & 1 & 9.5 & 18 & 806.589 & 0 & Circulator \\
\hline $04-25$ & 86 & 1460 & 1469 & $\mathrm{H} 2+\mathrm{SF} 6$ & 0.00004 & 1100 & 1 & 9.5 & 25 & 803.648 & 0 & Circulator \\
\hline $04-25$ & 86 & 1470 & 1479 & $\mathrm{H} 2+\mathrm{SF} 6$ & 0.00004 & 1100 & 1 & 9.5 & 20 & 803.657 & 0 & Circulator \\
\hline $04-25$ & 86 & 1480 & 1490 & $\mathrm{H} 2+\mathrm{SF} 6$ & 0.00004 & 1100 & 1 & 9.5 & 10 & 803.678 & 0 & Circulator \\
\hline $04-25$ & 86 & 1492 & 1501 & $\mathrm{H} 2+\mathrm{SF} 6$ & 0.00004 & 1100 & 1 & 9.5 & 5 & 803.685 & 0 & Circulator \\
\hline $04-25$ & 83 & 1136 & 1145 & $\mathrm{H} 2+\mathrm{SF} 6$ & 0.0002 & 1100 & 1 & 9.5 & 5 & 803.645 & 0 & Circulator \\
\hline $04-25$ & 83 & 1146 & 1165 & $\mathrm{H} 2+\mathrm{SF} 6$ & 0.0002 & 1100 & 1 & 9.5 & 10 & 803.649 & 0 & Circulator \\
\hline $04-25$ & 83 & 1166 & 1175 & $\mathrm{H} 2+\mathrm{SF} 6$ & 0.0002 & 1100 & 1 & 9.5 & 20 & 803.654 & 0 & Circulator \\
\hline $04-25$ & 83 & 1176 & 1186 & $\mathrm{H} 2+\mathrm{SF} 6$ & 0.0002 & 1100 & 1 & 9.5 & 25 & 803.649 & 0 & Circulator \\
\hline $04-25$ & 80 & 1003 & 1012 & $\mathrm{H} 2+\mathrm{SF} 6$ & 0.002 & 1100 & 1 & 9.5 & 25 & 803.571 & 0 & Circulator \\
\hline $04-25$ & 80 & 1014 & 1024 & $\mathrm{H} 2+\mathrm{SF} 6$ & 0.002 & 1100 & 1 & 9.5 & 20 & 803.562 & 0 & Circulator \\
\hline $04-25$ & 80 & 1025 & 1034 & $\mathrm{H} 2+\mathrm{SF} 6$ & 0.002 & 1100 & 1 & 9.5 & 10 & 803.576 & 0 & Circulator \\
\hline $04-25$ & 80 & 1035 & 1044 & $\mathrm{H} 2+\mathrm{SF} 6$ & 0.002 & 1100 & 1 & 9.5 & 5 & 803.594 & 0 & Circulator \\
\hline $04-25$ & 77 & 873 & 882 & $\mathrm{H} 2+\mathrm{SF} 6$ & 0.01 & 1100 & 1 & 9.5 & 25 & 803.44 & 0 & Circulator \\
\hline $04-25$ & 77 & 883 & 892 & $\mathrm{H} 2+\mathrm{SF} 6$ & 0.01 & 1100 & 1 & 9.5 & 20 & 803.447 & 0 & Circulator \\
\hline $04-25$ & 77 & 901 & 914 & $\mathrm{H} 2+\mathrm{SF} 6$ & 0.01 & 1100 & 1 & 9.5 & 10 & 803.483 & 0 & Circulator \\
\hline $04-25$ & 77 & 916 & 930 & $\mathrm{H} 2+\mathrm{SF} 6$ & 0.01 & 1100 & 1 & 9.5 & 5 & 803.508 & 0 & Circulator \\
\hline $04-25$ & 85 & 1417 & 1427 & $\mathrm{H} 2+\mathrm{SF} 6$ & 0.00004 & 1450 & 1 & 9.5 & 25 & 801.736 & 0 & Circulator \\
\hline $04-25$ & 85 & 1428 & 1437 & $\mathrm{H} 2+\mathrm{SF} 6$ & 0.00004 & 1450 & 1 & 9.5 & 20 & 801.745 & 0 & Circulator \\
\hline $04-25$ & 85 & 1438 & 1449 & $\mathrm{H} 2+\mathrm{SF} 6$ & 0.00004 & 1450 & 1 & 9.5 & 10 & 801.777 & 0 & Circulator \\
\hline $04-25$ & 85 & 1450 & 1459 & $\mathrm{H} 2+\mathrm{SF} 6$ & 0.00004 & 1450 & 1 & 9.5 & 5 & 801.789 & 0 & Circulator \\
\hline $04-25$ & 76 & 833 & 842 & $\mathrm{H} 2+\mathrm{SF} 6$ & 0.01 & 1450 & 1 & 9.5 & 25 & 801.501 & 0 & Circulator \\
\hline $04-25$ & 76 & 843 & 852 & $\mathrm{H} 2+\mathrm{SF} 6$ & 0.01 & 1450 & 1 & 9.5 & 20 & 801.494 & 0 & Circulator \\
\hline $04-25$ & 76 & 853 & 862 & $\mathrm{H} 2+\mathrm{SF} 6$ & 0.01 & 1450 & 1 & 9.5 & 10 & 801.489 & 0 & Circulator \\
\hline $04-25$ & 76 & 863 & 872 & $\mathrm{H} 2+\mathrm{SF} 6$ & 0.01 & 1450 & 1 & 9.5 & 5 & 801.488 & 0 & Circulator \\
\hline $04-25$ & 74 & 806 & 810 & $\mathrm{H} 2+\mathrm{SF} 6$ & 0.1 & 1450 & 1 & 9.5 & 25 & 800.101 & 0 & Circulator \\
\hline $04-25$ & 75 & 811 & 815 & $\mathrm{H} 2+\mathrm{SF} 6$ & 0.1 & 1450 & 1 & 9.5 & 25 & 799.911 & 0 & Circulator \\
\hline $04-25$ & 75 & 816 & 820 & $\mathrm{H} 2+\mathrm{SF} 6$ & 0.1 & 1450 & 1 & 9.5 & 20 & 799.892 & 0 & Circulator \\
\hline $04-25$ & 75 & 821 & 825 & $\mathrm{H} 2+\mathrm{SF} 6$ & 0.1 & 1450 & 1 & 9.5 & 10 & 799.881 & 0 & Circulator \\
\hline $04-25$ & 75 & 826 & 832 & $\mathrm{H} 2+\mathrm{SF} 6$ & 0.1 & 1450 & 1 & 9.5 & 5 & 799.86 & 0 & Circulator \\
\hline $04-25$ & 82 & 1077 & 1087 & $\mathrm{H} 2+\mathrm{SF} 6$ & 0.0002 & 1470 & 1 & 9.5 & 25 & 801.636 & 0 & Circulator \\
\hline $04-25$ & 82 & 1088 & 1111 & $\mathrm{H} 2+\mathrm{SF} 6$ & 0.0002 & 1470 & 1 & 9.5 & 20 & 801.642 & 0 & Circulator \\
\hline $04-25$ & 82 & 1112 & 1121 & $\mathrm{H} 2+\mathrm{SF} 6$ & 0.0002 & 1470 & 1 & 9.5 & 10 & 801.689 & 0 & Circulator \\
\hline $04-25$ & 82 & 1122 & 1135 & $\mathrm{H} 2+\mathrm{SF} 6$ & 0.0002 & 1470 & 1 & 9.5 & 5 & 801.695 & 0 & Circulator \\
\hline $04-25$ & 79 & 961 & 971 & $\mathrm{H} 2+\mathrm{SF} 6$ & 0.002 & 1470 & 1 & 9.5 & 25 & 801.577 & 0 & Circulator \\
\hline $04-25$ & 79 & 972 & 982 & $\mathrm{H} 2+\mathrm{SF} 6$ & 0.002 & 1470 & 1 & 9.5 & 20 & 801.587 & 0 & Circulator \\
\hline $04-25$ & 79 & 983 & 992 & $\mathrm{H} 2+\mathrm{SF} 6$ & 0.002 & 1470 & 1 & 9.5 & 10 & 801.603 & 0 & Circulator \\
\hline $04-25$ & 79 & 993 & 1002 & $\mathrm{H} 2+\mathrm{SF} 6$ & 0.002 & 1470 & 1 & 9.5 & 5 & 801.622 & 0 & Circulator \\
\hline 05-08 & 161 & 3778 & 3787 & $\mathrm{He}$ & 100 & 1470 & 1 & 9.5 & 8.9 & 808.545 & 0 & Hybr. \\
\hline
\end{tabular}




\begin{tabular}{|c|c|c|c|c|c|c|c|c|c|c|c|c|}
\hline Date & Run & $\begin{array}{l}\text { Start } \\
\text { Pulse }\end{array}$ & $\begin{array}{l}\text { End } \\
\text { Pulse }\end{array}$ & $\begin{array}{l}\text { Gas } \\
\text { Species }\end{array}$ & $\begin{array}{l}\text { Gas } \\
\text { Conc. } \\
(\%)\end{array}$ & $\begin{array}{l}\text { Gas } \\
\text { Press. } \\
\text { (psi) }\end{array}$ & $\begin{array}{l}\text { Beam } \\
\text { Int. }\end{array}$ & $\begin{array}{l}\text { Beam } \\
\text { Length } \\
(\mu \mathrm{s})\end{array}$ & $\begin{array}{l}\text { Elec. } \\
\text { Field } \\
(\mathrm{MV} / \mathrm{m})\end{array}$ & $\begin{array}{l}\text { Freq. } \\
(\mathrm{MHz})\end{array}$ & $\begin{array}{l}\text { Mag. } \\
\text { Field } \\
(\mathrm{T})\end{array}$ & $\begin{array}{l}\text { Circulator } \\
\text { /Hybr. }\end{array}$ \\
\hline 05-08 & 161 & 3788 & 3797 & $\mathrm{He}$ & 100 & 1470 & 1 & 9.5 & 5 & 808.55 & 0 & Hybr. \\
\hline 05-08 & 161 & 3798 & 3807 & $\mathrm{He}$ & 100 & 1470 & 0.2 & 9.5 & 5 & 808.55 & 0 & Hybr. \\
\hline 05-08 & 161 & 3808 & 3817 & $\mathrm{He}$ & 100 & 1470 & 0.2 & 9.5 & 8.9 & 808.559 & 0 & Hybr. \\
\hline 05-08 & 160 & 3698 & 3707 & $\mathrm{He}+\mathrm{Air}$ & 1 & 1470 & 1 & 9.5 & 25 & 808.124 & 0 & Hybr. \\
\hline 05-08 & 160 & 3708 & 3717 & $\mathrm{He}+\mathrm{Air}$ & 1 & 1470 & 1 & 9.5 & 20 & 808.131 & 0 & Hybr. \\
\hline 05-08 & 160 & 3718 & 3727 & $\mathrm{He}+\mathrm{Air}$ & 1 & 1470 & 1 & 9.5 & 10 & 808.148 & 0 & Hybr. \\
\hline 05-08 & 160 & 3728 & 3737 & $\mathrm{He}+\mathrm{Air}$ & 1 & 1470 & 1 & 9.5 & 5 & 808.157 & 0 & Hybr. \\
\hline 05-08 & 160 & 3738 & 3747 & $\mathrm{He}+\mathrm{Air}$ & 1 & 1470 & 0.2 & 9.5 & 5 & 808.157 & 0 & Hybr. \\
\hline 05-08 & 160 & 3748 & 3757 & $\mathrm{He}+\mathrm{Air}$ & 1 & 1470 & 0.2 & 9.5 & 10 & 808.166 & 0 & Hybr. \\
\hline 05-08 & 160 & 3758 & 3767 & $\mathrm{He}+\mathrm{Air}$ & 1 & 1470 & 0.2 & 9.5 & 20 & 808.163 & 0 & Hybr. \\
\hline 05-08 & 160 & 3768 & 3777 & $\mathrm{He}+\mathrm{Air}$ & 1 & 1470 & 0.2 & 9.5 & 25 & 808.15 & 0 & Hybr. \\
\hline $04-13$ & 1 & & & $\mathrm{~N} 2$ & 100 & 500 & & & & 803.372 & 0 & Circulator \\
\hline $04-13$ & 3 & & & $\mathrm{~N} 2$ & 100 & 500 & & & & 803.372 & 0 & Circulator \\
\hline $04-13$ & 4 & & & $\mathrm{~N} 2$ & 100 & 500 & & & & 803.381 & 0 & Circulator \\
\hline $04-13$ & 5 & & & $\mathrm{~N} 2$ & 100 & 500 & & & & 803.385 & 0 & Circulator \\
\hline 05-08 & 162 & 3818 & 3827 & $\mathrm{~N} 2$ & 100 & 700 & 1 & 9.5 & 25 & 800.477 & 0 & Hybr. \\
\hline 05-08 & 162 & 3828 & 3837 & $\mathrm{~N} 2$ & 100 & 700 & 1 & 9.5 & 20 & 800.477 & 0 & Hybr. \\
\hline 05-08 & 162 & 3838 & 3848 & $\mathrm{~N} 2$ & 100 & 700 & 1 & 9.5 & 10 & 800.491 & 0 & Hybr. \\
\hline 05-08 & 162 & 3849 & 3860 & $\mathrm{~N} 2$ & 100 & 700 & 1 & 9.5 & 5 & 800.507 & 0 & Hybr. \\
\hline 05-01 & 117 & 1788 & 1795 & $\mathrm{~N} 2+$ Air & 0.1 & 700 & 1 & 9.5 & 10 & 800.578 & 0 & Hybr. \\
\hline 05-01 & 116 & 1779 & 1787 & $\mathrm{~N} 2+$ Air & 1 & 700 & 1 & 9.5 & 10 & 800.525 & 0 & Hybr. \\
\hline 05-01 & 115 & 1771 & 1778 & $\mathrm{~N} 2+$ Air & 10 & 700 & 1 & 9.5 & 10 & 800.691 & 0 & Hybr. \\
\hline 05-01 & 114 & 1762 & 1770 & $\mathrm{~N} 2+\mathrm{Air}$ & 35.7 & 700 & 1 & 9.5 & 10 & 800.77 & 0 & Hybr. \\
\hline
\end{tabular}


APPENDIX C

CAVITY RESISTANCE 
Table C.1. Fitted time constants.

\begin{tabular}{|c|c|c|c|c|c|c|c|c|c|c|c|c|c|c|c|}
\hline Date & Run & $\begin{array}{l}\text { Start } \\
\text { Pulse }\end{array}$ & $\begin{array}{l}\text { End } \\
\text { Pulse }\end{array}$ & $\begin{array}{l}\text { Gas } \\
\text { Species }\end{array}$ & $\begin{array}{l}\text { Gas } \\
\text { Conc. } \\
(\%)\end{array}$ & $\begin{array}{l}\text { Gas } \\
\text { Press. } \\
\text { (psi) }\end{array}$ & $\begin{array}{l}\text { Beam } \\
\text { Int. }\end{array}$ & $\begin{array}{l}\text { Beam } \\
\text { Length } \\
(\mu \mathrm{s})\end{array}$ & $\begin{array}{l}\text { Elec. } \\
\text { Field } \\
(\mathrm{MV} / \mathrm{m})\end{array}$ & $\begin{array}{l}\text { Freq. } \\
(\mathrm{MHz})\end{array}$ & $\begin{array}{l}\text { Circ. } \\
\text { /Hybr. }\end{array}$ & $\mathrm{B} \tau(\mu \mathrm{s})$ & $\begin{array}{l}\text { B } \tau \text { erro } \\
(\mu \mathrm{s})\end{array}$ & $\mathrm{rE} \tau(\mu \mathrm{s})$ & $\begin{array}{l}\mathrm{E} \tau \text { error } \\
(\mu \mathrm{s})\end{array}$ \\
\hline $04 / 18$ & 17 & 47 & 58 & $\mathrm{H} 2$ & 100 & 800 & 1 & 9.5 & 10 & 805.5 & Circ. & 1.8797 & & & \\
\hline $04 / 18$ & 17 & 60 & 69 & $\mathrm{H} 2$ & 100 & 800 & 1 & 9.5 & 5 & 805.51 & Circ. & 1.9258 & & & \\
\hline $04 / 18$ & 23 & 0 & 7 & $\mathrm{H} 2$ & 100 & 800 & 0.2 & 9.5 & 5 & 805.55 & Circ. & 1.9258 & & & \\
\hline $04 / 18$ & 23 & 9 & 16 & $\mathrm{H} 2$ & 100 & 800 & 0.2 & 9.5 & 10 & 805.54 & Circ. & 1.8797 & & & \\
\hline $04 / 18$ & 24 & 24 & 33 & $\mathrm{H} 2$ & 100 & 800 & 0.1 & 9.5 & 10 & 805.55 & Circ. & 1.8797 & & & \\
\hline $04 / 18$ & 24 & 34 & 43 & $\mathrm{H} 2$ & 100 & 800 & 0.1 & 9.5 & 5 & 805.57 & Circ. & 1.9258 & & & \\
\hline $04 / 18$ & 24 & 44 & 48 & $\mathrm{H} 2$ & 100 & 800 & 0.05 & 9.5 & 10 & 805.57 & Circ. & 1.8797 & & & \\
\hline $04 / 19$ & 29 & 17 & 26 & $\mathrm{H} 2$ & 100 & 1100 & 1 & 7.5 & 25 & 803.67 & Circ. & 1.8799 & 0.0008 & 1.6482 & 0.0464 \\
\hline $04 / 19$ & 29 & 28 & 37 & $\mathrm{H} 2$ & 100 & 1100 & 1 & 7.5 & 20 & 803.67 & Circ. & 1.8799 & 0.0008 & 1.6482 & 0.0464 \\
\hline $04 / 19$ & 31 & 40 & 47 & $\mathrm{H} 2$ & 100 & 1100 & 1 & 7.5 & 10 & 803.73 & Circ. & 2.4086 & & & \\
\hline $04 / 19$ & 31 & 48 & 54 & $\mathrm{H} 2$ & 100 & 1100 & 1 & 7.5 & 5 & 803.74 & Circ. & 2.4325 & & & \\
\hline $04 / 19$ & 31 & 55 & 62 & $\mathrm{H} 2$ & 100 & 1100 & 0.2 & 7.5 & 5 & 803.74 & Circ. & 2.4325 & & & \\
\hline $04 / 19$ & 31 & 65 & 73 & $\mathrm{H} 2$ & 100 & 1100 & 0.2 & 7.5 & 10 & 803.76 & Circ. & 2.4086 & & & \\
\hline $04 / 19$ & 31 & 75 & 82 & $\mathrm{H} 2$ & 100 & 1100 & 0.2 & 7.5 & 20 & 803.76 & Circ. & 2.0944 & & & \\
\hline $04 / 19$ & 31 & 83 & 90 & $\mathrm{H} 2$ & 100 & 1100 & 0.2 & 7.5 & 25 & 803.76 & Circ. & 2.0586 & & & \\
\hline $04 / 19$ & 31 & 101 & 108 & $\mathrm{H} 2$ & 100 & 1100 & 0.1 & 7.5 & 25 & 803.757 & Circ. & 2.0586 & & & \\
\hline $04 / 19$ & 31 & 109 & 116 & $\mathrm{H} 2$ & 100 & 1100 & 0.1 & 7.5 & 20 & 803.76 & Circ. & 2.0944 & & & \\
\hline $04 / 19$ & 31 & 117 & 125 & $\mathrm{H} 2$ & 100 & 1100 & 0.1 & 7.5 & 10 & 803.78 & Circ. & 2.4086 & & & \\
\hline $04 / 19$ & 31 & 126 & 133 & $\mathrm{H} 2$ & 100 & 1100 & 0.1 & 7.5 & 5 & 803.79 & Circ. & 2.4325 & & & \\
\hline $04 / 19$ & 32 & 134 & 142 & $\mathrm{H} 2$ & 100 & 1520 & 0.1 & 7.5 & 25 & 801.3 & Circ. & 1.9239 & & & \\
\hline $04 / 19$ & 32 & 143 & 152 & $\mathrm{H} 2$ & 100 & 1520 & 0.1 & 7.5 & 20 & 801.3 & Circ. & 1.8396 & & & \\
\hline $04 / 19$ & 32 & 153 & 160 & $\mathrm{H} 2$ & 100 & 1520 & 0.1 & 7.5 & 10 & 801.3 & Circ. & 1.8143 & & & \\
\hline $04 / 19$ & 32 & 162 & 170 & $\mathrm{H} 2$ & 100 & 1520 & 0.1 & 7.5 & 5 & 801.331 & Circ. & 1.8619 & & & \\
\hline $04 / 19$ & 32 & 171 & 178 & $\mathrm{H} 2$ & 100 & 1520 & 1 & 7.5 & 5 & 801.35 & Circ. & 1.8619 & & & \\
\hline $04 / 19$ & 32 & 180 & 188 & $\mathrm{H} 2$ & 100 & 1520 & 1 & 7.5 & 10 & 801.36 & Circ. & 1.8143 & & & \\
\hline $04 / 19$ & 32 & 190 & 199 & $\mathrm{H} 2$ & 100 & 1520 & 1 & 7.5 & 20 & 801.366 & Circ. & 1.8396 & & & \\
\hline $04 / 19$ & 32 & 201 & 209 & $\mathrm{H} 2$ & 100 & 1520 & 1 & 7.5 & 25 & 801.37 & Circ. & 1.9239 & & & \\
\hline $04 / 19$ & 32 & 215 & 222 & $\mathrm{H} 2$ & 100 & 1520 & 0.2 & 7.5 & 25 & 801.374 & Circ. & 1.9239 & & & \\
\hline $04 / 19$ & 32 & 223 & 230 & $\mathrm{H} 2$ & 100 & 1520 & 0.2 & 7.5 & 20 & 801.384 & Circ. & 1.8396 & & & \\
\hline $04 / 19$ & 32 & 232 & 239 & $\mathrm{H} 2$ & 100 & 1520 & 0.2 & 7.5 & 10 & 801.398 & Circ. & 1.8143 & & & \\
\hline $04 / 19$ & 32 & 241 & 248 & $\mathrm{H} 2$ & 100 & 1520 & 0.2 & 7.5 & 5 & 801.419 & Circ. & 1.8619 & & & \\
\hline $04 / 20$ & 35 & 252 & 261 & $\mathrm{H} 2$ & 100 & 950 & 1 & 7.5 & 25 & 804.562 & Circ. & 1.8799 & 0.0008 & 1.8819 & 0.0007 \\
\hline $04 / 20$ & 35 & 263 & 271 & $\mathrm{H} 2$ & 100 & 950 & 1 & 7.5 & 20 & 804.57 & Circ. & 2.0058 & & & \\
\hline $04 / 20$ & 35 & 272 & 279 & $\mathrm{H} 2$ & 100 & 950 & 1 & 7.5 & 10 & 804.578 & Circ. & 2.0033 & 0.0058 & 1.9860 & 0.0055 \\
\hline $04 / 20$ & 35 & 281 & 289 & $\mathrm{H} 2$ & 100 & 950 & 1 & 7.5 & 5 & 804.598 & Circ. & 2.0037 & 0.0041 & 2.0173 & 0.0040 \\
\hline $04 / 20$ & 35 & 294 & 302 & $\mathrm{H} 2$ & 100 & 950 & 0.2 & 7.5 & 5 & 804.598 & Circ. & 1.9876 & 0.0050 & 2.0030 & 0.0036 \\
\hline $04 / 20$ & 35 & 305 & 313 & $\mathrm{H} 2$ & 100 & 950 & 0.2 & 7.5 & 10 & 804.617 & Circ. & 2.0012 & 0.0034 & 2.0247 & 0.0030 \\
\hline $04 / 20$ & 35 & 315 & 322 & $\mathrm{H} 2$ & 100 & 950 & 0.2 & 7.5 & 20 & 804.619 & Circ. & 1.9306 & 0.0018 & 1.9586 & 0.0018 \\
\hline
\end{tabular}




\begin{tabular}{|c|c|c|c|c|c|c|c|c|c|c|c|c|c|c|c|}
\hline Date & Run & $\begin{array}{l}\text { Start } \\
\text { Pulse }\end{array}$ & $\begin{array}{l}\text { End } \\
\text { Pulse }\end{array}$ & $\begin{array}{l}\text { Gas } \\
\text { Species }\end{array}$ & $\begin{array}{l}\text { Gas } \\
\text { Conc. } \\
(\%)\end{array}$ & $\begin{array}{l}\text { Gas } \\
\text { Press. } \\
\text { (psi) }\end{array}$ & $\begin{array}{l}\text { Beam } \\
\text { Int. }\end{array}$ & $\begin{array}{l}\text { Beam } \\
\text { Length } \\
(\mu \mathrm{s})\end{array}$ & $\begin{array}{l}\text { Elec. } \\
\text { Field } \\
(\mathrm{MV} / \mathrm{m})\end{array}$ & $\begin{array}{l}\text { Freq. } \\
(\mathrm{MHz})\end{array}$ & $\begin{array}{l}\text { Circ. } \\
\text { /Hybr. }\end{array}$ & $\mathrm{B} \tau(\mu \mathrm{s})$ & $\begin{array}{l}\text { B } \tau \text { error } \\
(\mu \mathrm{s})\end{array}$ & $\mathrm{rE} \tau(\mu \mathrm{s})$ & $\begin{array}{l}\mathrm{E} \tau \text { error } \\
(\mu \mathrm{s})\end{array}$ \\
\hline $04 / 20$ & 35 & 323 & 330 & $\mathrm{H} 2$ & 100 & 950 & 0.2 & 7.5 & 25 & 804.61 & Circ. & 1.8943 & 0.0015 & 1.9008 & 0.0016 \\
\hline $04 / 20$ & 35 & 334 & 341 & $\mathrm{H} 2$ & 100 & 950 & 0.1 & 7.5 & 25 & 804.61 & Circ. & 1.8922 & 0.0015 & 1.9041 & 0.0017 \\
\hline $04 / 20$ & 35 & 342 & 349 & $\mathrm{H} 2$ & 100 & 950 & 0.1 & 7.5 & 20 & 804.611 & Circ. & 1.9587 & 0.0020 & 1.9606 & 0.0020 \\
\hline $04 / 20$ & 35 & 350 & 357 & $\mathrm{H} 2$ & 100 & 950 & 0.1 & 7.5 & 10 & 804.625 & Circ. & 2.0316 & 0.0023 & 2.0435 & 0.0025 \\
\hline $04 / 20$ & 35 & 358 & 365 & $\mathrm{H} 2$ & 100 & 950 & 0.1 & 7.5 & 5 & 804.642 & Circ. & 2.0665 & & & \\
\hline $04 / 20$ & 36 & 367 & 374 & $\mathrm{H} 2$ & 100 & 1300 & 0.1 & 7.5 & 5 & 802.533 & Circ. & 1.9267 & 0.0027 & 1.9326 & 0.0029 \\
\hline $04 / 20$ & 36 & 377 & 384 & $\mathrm{H} 2$ & 100 & 1300 & 0.1 & 7.5 & 10 & 802.546 & Circ. & 1.9071 & 0.0022 & 1.9053 & 0.0024 \\
\hline $04 / 20$ & 36 & 385 & 392 & $\mathrm{H} 2$ & 100 & 1300 & 0.1 & 7.5 & 20 & 802.553 & Circ. & 1.8564 & 0.0026 & 1.8604 & 0.0030 \\
\hline $04 / 20$ & 36 & 393 & 401 & $\mathrm{H} 2$ & 100 & 1300 & 0.1 & 7.5 & 25 & 802.549 & Circ. & 1.8877 & & & \\
\hline $04 / 20$ & 36 & 409 & 416 & $\mathrm{H} 2$ & 100 & 1300 & 0.2 & 7.5 & 25 & 802.55 & Circ. & 1.8372 & 0.0024 & 1.8357 & 0.0028 \\
\hline $04 / 20$ & 36 & 417 & 424 & $\mathrm{H} 2$ & 100 & 1300 & 0.2 & 7.5 & 20 & 802.56 & Circ. & 1.8942 & 0.0025 & 1.8843 & 0.0026 \\
\hline $04 / 20$ & 36 & 425 & 432 & $\mathrm{H} 2$ & 100 & 1300 & 0.2 & 7.5 & 10 & 802.576 & Circ. & 1.9945 & 0.0025 & 1.9834 & 0.0026 \\
\hline $04 / 20$ & 36 & 433 & 440 & $\mathrm{H} 2$ & 100 & 1300 & 0.2 & 7.5 & 5 & 802.594 & Circ. & 2.0229 & & & \\
\hline $04 / 20$ & 36 & 445 & 452 & $\mathrm{H} 2$ & 100 & 1300 & 1 & 7.5 & 5 & 802.61 & Circ. & 2.0884 & 0.0028 & 2.0687 & 0.0029 \\
\hline $04 / 20$ & 36 & 453 & 461 & $\mathrm{H} 2$ & 100 & 1300 & 1 & 7.5 & 10 & 802.618 & Circ. & 2.0830 & 0.0025 & 2.0625 & 0.0025 \\
\hline $04 / 20$ & 36 & 462 & 469 & $\mathrm{H} 2$ & 100 & 1300 & 1 & 7.5 & 20 & 802.623 & Circ. & 2.0261 & 0.0025 & 2.0032 & 0.0027 \\
\hline $04 / 20$ & 36 & 470 & 477 & $\mathrm{H} 2$ & 100 & 1300 & 1 & 7.5 & 25 & 802.619 & Circ. & 1.4362 & 0.0539 & 1.5543 & 0.0504 \\
\hline $04 / 21$ & 37 & 478 & 485 & $\mathrm{H} 2$ & 100 & 300 & 1 & 7.5 & 5 & 808.525 & Circ. & 2.1263 & & & \\
\hline $04 / 21$ & 37 & 487 & 494 & $\mathrm{H} 2$ & 100 & 300 & 1 & 7.5 & 10 & 808.525 & Circ. & 2.1445 & & & \\
\hline $04 / 21$ & 37 & 495 & 502 & $\mathrm{H} 2$ & 100 & 300 & 1 & 7.5 & 18 & 808.517 & Circ. & 2.1721 & & & \\
\hline $04 / 21$ & 39 & 505 & 512 & $\mathrm{H} 2$ & 100 & 300 & 0.2 & 7.5 & 18 & 808.517 & Circ. & 2.1721 & & & \\
\hline $04 / 21$ & 39 & 540 & 546 & $\mathrm{H} 2$ & 100 & 300 & 0.2 & 7.5 & 10 & 808.51 & Circ. & 2.1445 & & & \\
\hline $04 / 21$ & 39 & 548 & 555 & $\mathrm{H} 2$ & 100 & 300 & 0.2 & 7.5 & 5 & 808.52 & Circ. & 2.1263 & & & \\
\hline $04 / 21$ & 39 & 557 & 564 & $\mathrm{H} 2$ & 100 & 300 & 0.1 & 7.5 & 5 & 808.522 & Circ. & 2.1263 & & & \\
\hline $04 / 21$ & 39 & 566 & 573 & $\mathrm{H} 2$ & 100 & 300 & 0.1 & 7.5 & 10 & 808.525 & Circ. & 2.1445 & & & \\
\hline $04 / 21$ & 39 & 575 & 582 & $\mathrm{H} 2$ & 100 & 300 & 0.1 & 7.5 & 18 & 808.521 & Circ. & 2.1721 & & & \\
\hline $04 / 21$ & 41 & 583 & 590 & $\mathrm{H} 2$ & 100 & 500 & 0.1 & 7.5 & 5 & 807.245 & Circ. & 2.5662 & & & \\
\hline $04 / 21$ & 41 & 596 & 603 & $\mathrm{H} 2$ & 100 & 500 & 0.1 & 7.5 & 10 & 807.25 & Circ. & 2.6326 & & & \\
\hline $04 / 21$ & 41 & 604 & 611 & $\mathrm{H} 2$ & 100 & 500 & 0.1 & 7.5 & 20 & 807.246 & Circ. & 2.6585 & & & \\
\hline $04 / 21$ & 41 & 612 & 619 & $\mathrm{H} 2$ & 100 & 500 & 0.1 & 7.5 & 25 & 807.231 & Circ. & 2.7185 & & & \\
\hline $04 / 21$ & 41 & 620 & 627 & $\mathrm{H} 2$ & 100 & 500 & 0.2 & 7.5 & 25 & 807.221 & Circ. & 2.7185 & & & \\
\hline $04 / 21$ & 41 & 630 & 637 & $\mathrm{H} 2$ & 100 & 500 & 0.2 & 7.5 & 20 & 807.229 & Circ. & 2.6585 & & & \\
\hline $04 / 21$ & 41 & 638 & 645 & $\mathrm{H} 2$ & 100 & 500 & 0.2 & 7.5 & 10 & 807.235 & Circ. & 2.6326 & & & \\
\hline $04 / 21$ & 41 & 646 & 653 & $\mathrm{H} 2$ & 100 & 500 & 0.2 & 7.5 & 5 & 807.244 & Circ. & 2.5662 & & & \\
\hline $04 / 21$ & 41 & 654 & 661 & $\mathrm{H} 2$ & 100 & 500 & 1 & 7.5 & 5 & 807.249 & Circ. & 2.5662 & & & \\
\hline $04 / 21$ & 41 & 662 & 669 & $\mathrm{H} 2$ & 100 & 500 & 1 & 7.5 & 10 & 807.254 & Circ. & 2.6326 & & & \\
\hline $04 / 21$ & 41 & 670 & 677 & $\mathrm{H} 2$ & 100 & 500 & 1 & 7.5 & 20 & 807.25 & Circ. & 2.6585 & & & \\
\hline $04 / 21$ & 41 & 678 & 685 & $\mathrm{H} 2$ & 100 & 500 & 1 & 7.5 & 25 & 807.254 & Circ. & 2.7185 & & & \\
\hline $04 / 22$ & 42 & 686 & 692 & $\mathrm{H} 2$ & 100 & 800 & 1 & 7.5 & 5 & 805.463 & Circ. & 1.9258 & & & \\
\hline $04 / 22$ & 42 & 693 & 700 & $\mathrm{H} 2$ & 100 & 800 & 1 & 7.5 & 10 & 805.466 & Circ. & 1.8797 & & & \\
\hline
\end{tabular}




\begin{tabular}{|c|c|c|c|c|c|c|c|c|c|c|c|c|c|c|c|}
\hline Date & Run & $\begin{array}{l}\text { Start } \\
\text { Pulse }\end{array}$ & $\begin{array}{l}\text { End } \\
\text { Pulse }\end{array}$ & $\begin{array}{l}\text { Gas } \\
\text { Species }\end{array}$ & $\begin{array}{l}\text { Gas } \\
\text { Conc. } \\
(\%)\end{array}$ & $\begin{array}{l}\text { Gas } \\
\text { Press. } \\
\text { (psi) }\end{array}$ & $\begin{array}{l}\text { Beam } \\
\text { Int. }\end{array}$ & $\begin{array}{l}\text { Beam } \\
\text { Length } \\
(\mu \mathrm{s})\end{array}$ & $\begin{array}{l}\text { Elec. } \\
\text { Field } \\
(\mathrm{MV} / \mathrm{m})\end{array}$ & $\begin{array}{l}\text { Freq. } \\
(\mathrm{MHz})\end{array}$ & $\begin{array}{l}\text { Circ. } \\
\text { /Hybr. }\end{array}$ & $\mathrm{B} \tau(\mu \mathrm{s})$ & $\begin{array}{l}\text { B } \tau \text { erro } \\
(\mu \mathrm{s})\end{array}$ & $\mathrm{rE} \tau(\mu \mathrm{s})$ & $\begin{array}{l}\mathrm{E} \tau \text { error } \\
(\mu \mathrm{s})\end{array}$ \\
\hline $04 / 22$ & 42 & 709 & 716 & $\mathrm{H} 2$ & 100 & 800 & 1 & 7.5 & 25 & 805.458 & Circ. & 1.8238 & & & \\
\hline $04 / 22$ & 42 & 720 & 727 & $\mathrm{H} 2$ & 100 & 800 & 0.2 & 7.5 & 25 & 805.458 & Circ. & 1.8238 & & & \\
\hline $04 / 22$ & 42 & 736 & 743 & $\mathrm{H} 2$ & 100 & 800 & 0.2 & 7.5 & 10 & 805.47 & Circ. & 1.8797 & & & \\
\hline $04 / 22$ & 42 & 744 & 751 & $\mathrm{H} 2$ & 100 & 800 & 0.2 & 7.5 & 5 & 805.482 & Circ. & 1.9258 & & & \\
\hline $04 / 22$ & 42 & 754 & 761 & $\mathrm{H} 2$ & 100 & 800 & 0.1 & 7.5 & 5 & 805.482 & Circ. & 1.9258 & & & \\
\hline $04 / 22$ & 42 & 762 & 769 & $\mathrm{H} 2$ & 100 & 800 & 0.1 & 7.5 & 10 & 805.495 & Circ. & 1.8797 & & & \\
\hline $04 / 22$ & 42 & 780 & 787 & $\mathrm{H} 2$ & 100 & 800 & 0.1 & 7.5 & 25 & 805.486 & Circ. & 1.8238 & & & \\
\hline $04 / 23$ & 44 & 17 & 24 & $\mathrm{H} 2$ & 100 & 1520 & 0.05 & 7.5 & 25 & 801.27 & Circ. & 1.9239 & 0.0019 & 1.9051 & 0.0013 \\
\hline $04 / 23$ & 44 & 26 & 32 & $\mathrm{H} 2$ & 100 & 1520 & 0.05 & 7.5 & 20 & 801.256 & Circ. & 1.8396 & 0.0030 & 1.8319 & 0.0024 \\
\hline $04 / 23$ & 44 & 34 & 41 & $\mathrm{H} 2$ & 100 & 1520 & 0.05 & 7.5 & 10 & 801.284 & Circ. & 1.8143 & 0.0019 & & \\
\hline $04 / 23$ & 44 & 50 & 57 & $\mathrm{H} 2$ & 100 & 1520 & 0.05 & 7.5 & 5 & 801.338 & Circ. & 1.8619 & 0.0031 & 1.8723 & 0.0030 \\
\hline $04 / 23$ & 45 & 63 & 64 & $\mathrm{H} 2$ & 100 & 1300 & 0.05 & 7.5 & 5 & 802.518 & Circ. & 2.0229 & & & \\
\hline $04 / 23$ & 47 & 69 & 76 & $\mathrm{H} 2$ & 100 & 1300 & 0.05 & 7.5 & 5 & 802.527 & Circ. & 2.0538 & 0.0028 & 2.0643 & 0.0026 \\
\hline $04 / 23$ & 47 & 80 & 87 & $\mathrm{H} 2$ & 100 & 1300 & 0.05 & 7.5 & 10 & 802.551 & Circ. & 2.0526 & 0.0029 & 2.0594 & 0.0027 \\
\hline $04 / 23$ & 47 & 89 & 96 & $\mathrm{H} 2$ & 100 & 1300 & 0.05 & 7.5 & 20 & 802.558 & Circ. & 2.0014 & 0.0032 & 2.0136 & 0.0030 \\
\hline $04 / 23$ & 47 & 98 & 105 & $\mathrm{H} 2$ & 100 & 1300 & 0.05 & 7.5 & 25 & 802.559 & Circ. & 2.2951 & 0.0038 & 2.3417 & 0.0058 \\
\hline $04 / 23$ & 49 & 111 & 121 & $\mathrm{H} 2$ & 100 & 1100 & 0.05 & 7.5 & 25 & 803.71 & Circ. & 2.2364 & 0.0028 & 2.2340 & 0.0019 \\
\hline $04 / 23$ & 49 & 123 & 130 & $\mathrm{H} 2$ & 100 & 1100 & 0.05 & 7.5 & 20 & 803.703 & Circ. & 2.3088 & 0.0029 & 2.3169 & 0.0023 \\
\hline $04 / 23$ & 49 & 132 & 139 & $\mathrm{H} 2$ & 100 & 1100 & 0.05 & 7.5 & 10 & 803.719 & Circ. & 2.4086 & 0.0028 & 2.3940 & 0.0022 \\
\hline $04 / 23$ & 49 & 141 & 148 & $\mathrm{H} 2$ & 100 & 1100 & 0.05 & 7.5 & 5 & 803.732 & Circ. & 2.4325 & 0.0027 & 2.4384 & 0.0023 \\
\hline $04 / 23$ & 50 & 150 & 158 & $\mathrm{H} 2$ & 100 & 950 & 0.05 & 7.5 & 5 & 804.628 & Circ. & 2.2082 & 0.0020 & 2.2025 & 0.0016 \\
\hline $04 / 23$ & 50 & 160 & 167 & $\mathrm{H} 2$ & 100 & 950 & 0.05 & 7.5 & 10 & 804.637 & Circ. & 2.1928 & 0.0019 & 2.1924 & 0.0016 \\
\hline $04 / 23$ & 50 & 172 & 180 & H2 & 100 & 950 & 0.05 & 7.5 & 20 & 804.634 & Circ. & 2.1283 & 0.0022 & 2.1248 & 0.0019 \\
\hline $04 / 23$ & 50 & 182 & 189 & $\mathrm{H} 2$ & 100 & 950 & 0.05 & 7.5 & 25 & 804.629 & Circ. & 2.0758 & 0.0019 & 2.0671 & 0.0018 \\
\hline $04 / 23$ & 51 & 191 & 203 & $\mathrm{H} 2$ & 100 & 800 & 0.05 & 7.5 & 25 & 805.413 & Circ. & 1.8238 & 0.0024 & 1.8486 & 0.0020 \\
\hline $04 / 23$ & 51 & 213 & 220 & $\mathrm{H} 2$ & 100 & 800 & 0.05 & 7.5 & 10 & 805.4 & Circ. & 1.8658 & 0.0024 & & \\
\hline $04 / 23$ & 53 & 226 & 226 & $\mathrm{H} 2$ & 100 & 800 & 0.05 & 7.5 & 10 & 805.4 & Circ. & 1.8936 & 0.0016 & 1.9116 & 0.0010 \\
\hline $04 / 23$ & 53 & 227 & 234 & $\mathrm{H} 2$ & 100 & 800 & 0.05 & 7.5 & 5 & 805.414 & Circ. & 1.9258 & 0.0016 & 1.9458 & 0.0011 \\
\hline $04 / 23$ & 54 & 237 & 244 & $\mathrm{H} 2$ & 100 & 500 & 0.05 & 7.5 & 5 & 807.24 & Circ. & 2.5662 & 0.0031 & 2.5610 & 0.0025 \\
\hline $04 / 23$ & 54 & 246 & 253 & $\mathrm{H} 2$ & 100 & 500 & 0.05 & 7.5 & 10 & 807.238 & Circ. & 2.6326 & 0.0037 & 2.6274 & 0.0028 \\
\hline $04 / 23$ & 54 & 255 & 262 & $\mathrm{H} 2$ & 100 & 500 & 0.05 & 7.5 & 20 & 807.234 & Circ. & 2.6585 & 0.0048 & 2.6920 & 0.0032 \\
\hline $04 / 23$ & 54 & 265 & 272 & $\mathrm{H} 2$ & 100 & 500 & 0.05 & 7.5 & 25 & 807.221 & Circ. & 2.7185 & 0.0043 & 2.7261 & 0.0030 \\
\hline $04 / 23$ & 55 & 275 & 282 & $\mathrm{H} 2$ & 100 & 300 & 0.05 & 7.5 & 5 & 808.491 & Circ. & 2.1263 & 0.0029 & 2.1288 & 0.0023 \\
\hline $04 / 23$ & 55 & 286 & 293 & $\mathrm{H} 2$ & 100 & 300 & 0.05 & 7.5 & 10 & 808.492 & Circ. & 2.1445 & 0.0034 & 2.1426 & 0.0032 \\
\hline $04 / 23$ & 55 & 296 & 303 & $\mathrm{H} 2$ & 100 & 300 & 0.05 & 7.5 & 18 & 808.489 & Circ. & 2.1721 & 0.1463 & 2.1779 & 0.1513 \\
\hline $04 / 26$ & 88 & 1530 & 1538 & H2 & 100 & 1100 & 1 & 9.5 & 25 & 803.679 & Circ. & 2.0594 & 0.0019 & 2.0643 & 0.0014 \\
\hline $04 / 26$ & 91 & 1548 & 1552 & $\mathrm{H} 2$ & 100 & 1100 & 1 & 9.5 & 25 & 803.783 & Circ. & 2.0586 & & & \\
\hline $04 / 26$ & 92 & 1555 & 1562 & $\mathrm{H} 2$ & 100 & 1470 & 1 & 9.5 & 25 & 801.635 & Circ. & 2.4281 & & & \\
\hline $04 / 27$ & 104 & 1773 & 1780 & $\mathrm{H} 2$ & 100 & 1470 & 1 & 9.5 & 25 & 801.629 & Circ. & 2.4281 & 0.0024 & 2.4391 & 0.0019 \\
\hline $04 / 27$ & 104 & 1783 & 1791 & $\mathrm{H} 2$ & 100 & 1470 & 1 & 9.5 & 20 & 801.63 & Circ. & 2.4810 & 0.0024 & 2.5058 & 0.0020 \\
\hline
\end{tabular}




\begin{tabular}{|c|c|c|c|c|c|c|c|c|c|c|c|c|c|c|c|}
\hline Date & Run & $\begin{array}{l}\text { Start } \\
\text { Pulse }\end{array}$ & $\begin{array}{l}\text { End } \\
\text { Pulse }\end{array}$ & $\begin{array}{l}\text { Gas } \\
\text { Species }\end{array}$ & $\begin{array}{l}\text { Gas } \\
\text { Conc. } \\
(\%)\end{array}$ & $\begin{array}{l}\text { Gas } \\
\text { Press. } \\
\text { (psi) }\end{array}$ & $\begin{array}{l}\text { Beam } \\
\text { Int. }\end{array}$ & $\begin{array}{l}\text { Beam } \\
\text { Length } \\
(\mu \mathrm{s})\end{array}$ & $\begin{array}{l}\text { Elec. } \\
\text { Field } \\
(\mathrm{MV} / \mathrm{m})\end{array}$ & $\begin{array}{l}\text { Freq. } \\
(\mathrm{MHz})\end{array}$ & $\begin{array}{l}\text { Circ. } \\
\text { /Hybr. }\end{array}$ & $\mathrm{B} \tau(\mu \mathrm{s})$ & $\begin{array}{l}\mathrm{B} \tau \text { error } \\
(\mu \mathrm{s})\end{array}$ & $\mathrm{rE} \tau(\mu \mathrm{s})$ & $\begin{array}{l}\mathrm{E} \tau \text { error } \\
(\mu \mathrm{s})\end{array}$ \\
\hline $04 / 27$ & 104 & 1794 & 1801 & $\mathrm{H} 2$ & 100 & 1470 & 1 & 9.5 & 10 & 801.65 & Circ. & 2.5312 & 0.0019 & 2.5468 & 0.0023 \\
\hline $04 / 27$ & 104 & 1804 & 1811 & $\mathrm{H} 2$ & 100 & 1470 & 1 & 9.5 & 5 & 801.673 & Circ. & 2.5646 & 0.0019 & 2.5728 & 0.0016 \\
\hline $04 / 30$ & 107 & 1814 & 1822 & $\mathrm{H} 2$ & 100 & 1470 & 1 & 9.5 & 50 & 801.646 & Hybr. & 3.9090 & 0.0101 & 3.9256 & 0.0083 \\
\hline $04 / 30$ & 107 & 1825 & 1842 & $\mathrm{H} 2$ & 100 & 1470 & 1 & 9.5 & 40 & 801.637 & Hybr. & 3.6899 & 0.0087 & 3.6868 & 0.0072 \\
\hline $04 / 30$ & 107 & 1843 & 1857 & $\mathrm{H} 2$ & 100 & 1470 & 1 & 9.5 & 40 & 801.708 & Hybr. & 3.6899 & 0.0087 & 3.6868 & 0.0072 \\
\hline $04 / 30$ & 107 & 1862 & 1872 & $\mathrm{H} 2$ & 100 & 1470 & 0.1 & 9.5 & 40 & 801.751 & Hybr. & 3.5035 & 0.0077 & 3.4915 & 0.0067 \\
\hline $04 / 30$ & 107 & 1876 & 1887 & $\mathrm{H} 2$ & 100 & 1470 & 0.1 & 9.5 & 50 & 801.761 & Hybr. & 3.5071 & & & \\
\hline $04 / 30$ & 109 & 1888 & 1888 & $\mathrm{H} 2$ & 100 & 1470 & 0.1 & 9.5 & 50 & 801.761 & Hybr. & 3.3184 & 0.0106 & 3.3438 & 0.0086 \\
\hline $04 / 30$ & 111 & 1890 & 1894 & $\mathrm{H} 2$ & 100 & 1470 & 0.1 & 9.5 & 50 & 801.761 & Hybr. & 3.2938 & 0.0103 & 3.2829 & 0.0090 \\
\hline 05/01 & 112 & 1555 & 1562 & $\mathrm{H} 2$ & 100 & 1100 & 1 & 9.5 & 40 & 803.529 & Hybr. & 3.6193 & 0.0109 & 3.6325 & 0.0118 \\
\hline 05/01 & 112 & 1565 & 1573 & $\mathrm{H} 2$ & 100 & 1100 & 1 & 9.5 & 30 & 803.557 & Hybr. & 3.8138 & 0.0103 & 3.8218 & 0.0117 \\
\hline 05/01 & 112 & 1708 & 1715 & $\mathrm{H} 2$ & 100 & 1100 & 0.1 & 9.5 & 40 & 803.564 & Hybr. & 3.7039 & 0.0072 & 3.7181 & 0.0070 \\
\hline $05 / 01$ & 113 & 1718 & 1725 & $\mathrm{H} 2$ & 100 & 950 & 0.1 & 9.5 & 40 & 804.575 & Hybr. & 3.6667 & 0.0149 & 3.6176 & 0.0160 \\
\hline $05 / 01$ & 113 & 1728 & 1737 & $\mathrm{H} 2$ & 100 & 950 & 0.1 & 9.5 & 30 & 804.545 & Hybr. & 3.9134 & 0.0121 & 3.8904 & 0.0137 \\
\hline $05 / 01$ & 113 & 1740 & 1750 & $\mathrm{H} 2$ & 100 & 950 & 1 & 9.5 & 30 & 804.563 & Hybr. & 3.9280 & 0.0231 & 3.8827 & 0.0232 \\
\hline 05/01 & 113 & 1753 & 1760 & $\mathrm{H} 2$ & 100 & 950 & 1 & 9.5 & 40 & 804.577 & Hybr. & 3.7213 & 0.0149 & & \\
\hline $05 / 02$ & 118 & 1796 & 1800 & $\mathrm{H} 2$ & 100 & 1470 & 1 & 9.5 & 50 & 801.546 & Hybr. & 3.5071 & & & \\
\hline $05 / 02$ & 125 & 2185 & 2190 & $\mathrm{H} 2$ & 100 & 300 & 1 & 9.5 & 10 & 808.432 & Hybr. & 1.7549 & 0.0061 & 1.7748 & 0.0060 \\
\hline $05 / 07$ & 157 & 3568 & 3575 & $\mathrm{H} 2$ & 100 & 1140 & 0.05 & 9.5 & 25 & 803.485 & Hybr. & 3.6564 & 0.0139 & 3.7094 & 0.0151 \\
\hline $05 / 07$ & 157 & 3578 & 3585 & $\mathrm{H} 2$ & 100 & 1140 & 0.05 & 9.5 & 20 & 803.483 & Hybr. & 3.7296 & 0.0159 & 3.7587 & 0.0172 \\
\hline $05 / 07$ & 157 & 3588 & 3595 & $\mathrm{H} 2$ & 100 & 1140 & 0.05 & 9.5 & 10 & 803.495 & Hybr. & 3.8270 & 0.0194 & 3.8280 & 0.0216 \\
\hline $05 / 07$ & 157 & 3598 & 3605 & $\mathrm{H} 2$ & 100 & 1140 & 0.05 & 9.5 & 5 & 803.509 & Hybr. & 3.8450 & 0.0136 & 3.8663 & 0.0149 \\
\hline $05 / 07$ & 158 & 3608 & 3615 & $\mathrm{H} 2$ & 100 & 800 & 0.05 & 9.5 & 25 & 805.384 & Hybr. & & & & \\
\hline $05 / 07$ & 158 & 3618 & 3625 & $\mathrm{H} 2$ & 100 & 800 & 0.05 & 9.5 & 20 & 805.373 & Hybr. & 2.8069 & 0.0242 & 2.8298 & 0.0235 \\
\hline $05 / 07$ & 158 & 3628 & 3635 & $\mathrm{H} 2$ & 100 & 800 & 0.05 & 9.5 & 10 & 805.383 & Hybr. & 2.9282 & 0.0257 & 2.9213 & 0.0241 \\
\hline $05 / 07$ & 158 & 3638 & 3645 & $\mathrm{H} 2$ & 100 & 800 & 0.05 & 9.5 & 5 & 805.398 & Hybr. & 3.0319 & 0.0197 & 3.0470 & 0.0192 \\
\hline $05 / 07$ & 159 & 3648 & 3655 & $\mathrm{H} 2$ & 100 & 500 & 0.05 & 9.5 & 25 & 807.211 & Hybr. & 1.9618 & 0.0057 & 1.9633 & 0.0057 \\
\hline $05 / 07$ & 159 & 3658 & 3665 & $\mathrm{H} 2$ & 100 & 500 & 0.05 & 9.5 & 20 & 807.211 & Hybr. & 1.9232 & 0.0053 & 1.9204 & 0.0056 \\
\hline $05 / 07$ & 159 & 3668 & 3675 & $\mathrm{H} 2$ & 100 & 500 & 0.05 & 9.5 & 10 & 807.219 & Hybr. & 1.9189 & 0.0049 & 1.9188 & 0.0047 \\
\hline $05 / 07$ & 159 & 3678 & 3685 & $\mathrm{H} 2$ & 100 & 500 & 0.05 & 9.5 & 5 & 807.235 & Hybr. & 1.8663 & 0.0048 & 1.8615 & 0.0046 \\
\hline $04 / 24$ & 58 & 307 & 315 & $\mathrm{H} 2+\mathrm{Air}$ & 10 & 735 & 1 & 9.5 & 25 & 805.037 & Circ. & 2.8072 & 0.0048 & 2.7990 & 0.0037 \\
\hline $04 / 24$ & 58 & 322 & 329 & $\mathrm{H} 2+\mathrm{Air}$ & 10 & 735 & 1 & 9.5 & 20 & 805.034 & Circ. & 2.7721 & 0.0038 & 2.7791 & 0.0038 \\
\hline $04 / 24$ & 58 & 332 & 339 & $\mathrm{H} 2+\mathrm{Air}$ & 10 & 735 & 1 & 9.5 & 10 & 805.06 & Circ. & 2.6892 & 0.0036 & 2.6878 & 0.0030 \\
\hline $04 / 24$ & 58 & 341 & 348 & $\mathrm{H} 2+\mathrm{Air}$ & 10 & 735 & 1 & 9.5 & 5 & 805.08 & Circ. & 2.6172 & 0.0045 & 2.6224 & 0.0042 \\
\hline $04 / 24$ & 59 & 351 & 358 & $\mathrm{H} 2+\mathrm{Air}$ & 6.78 & 1100 & 1 & 9.5 & 25 & 802.921 & Circ. & 2.7856 & 0.0049 & 2.7862 & 0.0034 \\
\hline $04 / 24$ & 59 & 362 & 370 & $\mathrm{H} 2+\mathrm{Air}$ & 6.78 & 1100 & 1 & 9.5 & 20 & 802.924 & Circ. & 2.6855 & 0.0036 & 2.7216 & 0.0028 \\
\hline $04 / 24$ & 59 & 373 & 380 & $\mathrm{H} 2+\mathrm{Air}$ & 6.78 & 1100 & 1 & 9.5 & 10 & 802.947 & Circ. & 2.6050 & 0.0038 & 2.6578 & 0.0034 \\
\hline $04 / 24$ & 59 & 383 & 390 & $\mathrm{H} 2+\mathrm{Air}$ & 6.78 & 1100 & 1 & 9.5 & 5 & 802.971 & Circ. & 2.5622 & 0.0025 & 2.5789 & 0.0020 \\
\hline $04 / 24$ & 61 & 393 & 401 & $\mathrm{H} 2+\mathrm{Air}$ & 5 & 1450 & 1 & 9.5 & 25 & 800.884 & Circ. & 2.6985 & 0.0040 & 2.6923 & 0.0035 \\
\hline $04 / 24$ & 61 & 404 & 411 & $\mathrm{H} 2+\mathrm{Air}$ & 5 & 1450 & 1 & 9.5 & 20 & 800.894 & Circ. & 2.6342 & 0.0036 & 2.6382 & 0.0033 \\
\hline
\end{tabular}




\begin{tabular}{|c|c|c|c|c|c|c|c|c|c|c|c|c|c|c|c|}
\hline Date & Run & $\begin{array}{l}\text { Start } \\
\text { Pulse }\end{array}$ & $\begin{array}{l}\text { End } \\
\text { Pulse }\end{array}$ & $\begin{array}{l}\text { Gas } \\
\text { Species }\end{array}$ & $\begin{array}{l}\text { Gas } \\
\text { Conc. } \\
(\%)\end{array}$ & $\begin{array}{l}\text { Gas } \\
\text { Press. } \\
\text { (psi) }\end{array}$ & $\begin{array}{l}\text { Beam } \\
\text { Int. }\end{array}$ & $\begin{array}{l}\text { Beam } \\
\text { Length } \\
(\mu \mathrm{s})\end{array}$ & $\begin{array}{l}\text { Elec. } \\
\text { Field } \\
(\mathrm{MV} / \mathrm{m})\end{array}$ & $\begin{array}{l}\text { Freq. } \\
(\mathrm{MHz})\end{array}$ & $\begin{array}{l}\text { Circ. } \\
\text { /Hybr. }\end{array}$ & $\mathrm{B} \tau(\mu \mathrm{s})$ & $\begin{array}{l}\text { B } \tau \text { errol } \\
(\mu \mathrm{s})\end{array}$ & $\mathrm{rE} \tau(\mu \mathrm{s})$ & $\begin{array}{l}\mathrm{E} \tau \text { error } \\
(\mu \mathrm{s})\end{array}$ \\
\hline $04 / 24$ & 61 & 414 & 422 & $\mathrm{H} 2+\mathrm{Air}$ & 5 & 1450 & 1 & 9.5 & 10 & 800.911 & Circ. & 2.5333 & 0.0034 & 2.5610 & 0.0038 \\
\hline $04 / 24$ & 61 & 425 & 433 & $\mathrm{H} 2+\mathrm{Air}$ & 5 & 1450 & 1 & 9.5 & 5 & 800.93 & Circ. & 2.5009 & 0.0025 & 2.5541 & 0.0029 \\
\hline $04 / 24$ & 62 & 436 & 444 & $\mathrm{H} 2+\mathrm{Air}$ & 5 & 1100 & 1 & 9.5 & 25 & 803.006 & Circ. & 2.6412 & 0.0034 & 2.6421 & 0.0024 \\
\hline $04 / 24$ & 62 & 446 & 454 & $\mathrm{H} 2+\mathrm{Air}$ & 5 & 1100 & 1 & 9.5 & 20 & 803.007 & Circ. & 2.5566 & 0.0032 & 2.5769 & 0.0024 \\
\hline $04 / 24$ & 62 & 458 & 464 & $\mathrm{H} 2+\mathrm{Air}$ & 5 & 1100 & 1 & 9.5 & 10 & 803.019 & Circ. & 2.5543 & 0.0023 & 2.5757 & 0.0018 \\
\hline $04 / 24$ & 62 & 467 & 474 & $\mathrm{H} 2+\mathrm{Air}$ & 5 & 1100 & 1 & 9.5 & 5 & 803.038 & Circ. & 2.5026 & 0.0018 & 2.5194 & 0.0015 \\
\hline $04 / 24$ & 65 & 478 & 486 & $\mathrm{H} 2+\mathrm{Air}$ & 5 & 300 & 1 & 9.5 & 5 & 808.34 & Circ. & 2.3483 & 0.0024 & 2.3587 & 0.0021 \\
\hline $04 / 24$ & 65 & 490 & 497 & $\mathrm{H} 2+\mathrm{Air}$ & 5 & 300 & 1 & 9.5 & 10 & 808.34 & Circ. & 2.3490 & 0.0028 & 2.3464 & 0.0029 \\
\hline $04 / 24$ & 65 & 500 & 510 & $\mathrm{H} 2+\mathrm{Air}$ & 5 & 300 & 1 & 9.5 & 18 & 808.335 & Circ. & 2.3649 & 0.0034 & 2.3768 & 0.0025 \\
\hline $04 / 24$ & 66 & 514 & 522 & $\mathrm{H} 2+\mathrm{Air}$ & 1 & 1470 & 1 & 9.5 & 25 & 801.46 & Circ. & 1.8846 & 0.0032 & 1.8966 & 0.0033 \\
\hline $04 / 24$ & 66 & 525 & 532 & $\mathrm{H} 2+\mathrm{Air}$ & 1 & 1470 & 1 & 9.5 & 20 & 801.45 & Circ. & 1.9152 & 0.0031 & & \\
\hline $04 / 24$ & 66 & 535 & 542 & $\mathrm{H} 2+\mathrm{Air}$ & 1 & 1470 & 1 & 9.5 & 10 & 801.457 & Circ. & 2.0012 & 0.0027 & 2.0114 & 0.0030 \\
\hline $04 / 24$ & 66 & 545 & 552 & $\mathrm{H} 2+\mathrm{Air}$ & 1 & 1470 & 1 & 9.5 & 5 & 801.471 & Circ. & 2.0538 & 0.0025 & 2.0700 & 0.0026 \\
\hline $04 / 24$ & 67 & 555 & 562 & $\mathrm{H} 2+\mathrm{Air}$ & 1 & 1100 & 1 & 9.5 & 25 & 803.473 & Circ. & 1.7497 & 0.0016 & 1.7392 & 0.0011 \\
\hline $04 / 24$ & 67 & 566 & 573 & $\mathrm{H} 2+\mathrm{Air}$ & 1 & 1100 & 1 & 9.5 & 20 & 803.474 & Circ. & 1.7636 & 0.0022 & 1.7511 & 0.0015 \\
\hline $04 / 24$ & 67 & 576 & 583 & $\mathrm{H} 2+\mathrm{Air}$ & 1 & 1100 & 1 & 9.5 & 10 & 803.486 & Circ. & 1.8308 & 0.0017 & 1.8151 & 0.0019 \\
\hline $04 / 24$ & 67 & 586 & 593 & $\mathrm{H} 2+$ Air & 1 & 1100 & 1 & 9.5 & 5 & 803.487 & Circ. & 1.8767 & 0.0015 & 1.8740 & 0.0010 \\
\hline $04 / 24$ & 68 & 597 & 604 & $\mathrm{H} 2+\mathrm{Air}$ & 1 & 300 & 1 & 9.5 & 18 & 808.461 & Circ. & 2.1017 & 0.0035 & 2.1022 & 0.0025 \\
\hline $04 / 24$ & 68 & 608 & 615 & H2+Air & 1 & 300 & 1 & 9.5 & 10 & 808.448 & Circ. & 2.1230 & 0.0028 & 2.1271 & 0.0024 \\
\hline $04 / 24$ & 68 & 618 & 625 & $\mathrm{H} 2+\mathrm{Air}$ & 1 & 300 & 1 & 9.5 & 5 & 808.452 & Circ. & 2.0762 & 0.0023 & 2.0847 & 0.0020 \\
\hline $04 / 24$ & 69 & 628 & 638 & $\mathrm{H} 2+\mathrm{Air}$ & 0.2 & 1450 & 1 & 9.5 & 25 & 801.642 & Circ. & 2.4230 & 0.0026 & 2.4324 & 0.0020 \\
\hline $04 / 24$ & 69 & 641 & 648 & $\mathrm{H} 2+\mathrm{Air}$ & 0.2 & 1450 & 1 & 9.5 & 20 & 801.637 & Circ. & 2.4867 & 0.0023 & 2.5120 & 0.0019 \\
\hline $04 / 24$ & 69 & 651 & 657 & $\mathrm{H} 2+\mathrm{Air}$ & 0.2 & 1450 & 1 & 9.5 & 10 & 801.65 & Circ. & 2.5371 & 0.0018 & 2.5512 & 0.0022 \\
\hline $04 / 24$ & 69 & 660 & 668 & $\mathrm{H} 2+\mathrm{Air}$ & 0.2 & 1450 & 1 & 9.5 & 5 & 801.773 & Circ. & 2.5608 & 0.0018 & 2.5712 & 0.0013 \\
\hline $04 / 24$ & 70 & 672 & 682 & $\mathrm{H} 2+\mathrm{Air}$ & 0.2 & 300 & 1 & 9.5 & 18 & 808.316 & Circ. & 2.5545 & 0.0029 & 2.5827 & 0.0028 \\
\hline $04 / 24$ & 70 & 686 & 694 & $\mathrm{H} 2+\mathrm{Air}$ & 0.2 & 300 & 1 & 9.5 & 10 & 808.22 & Circ. & 2.6569 & 0.0024 & 2.6643 & 0.0027 \\
\hline $04 / 24$ & 70 & 698 & 706 & $\mathrm{H} 2+\mathrm{Air}$ & 0.2 & 300 & 1 & 9.5 & 5 & 808.188 & Circ. & 2.6294 & 0.0023 & 2.6208 & 0.0018 \\
\hline $04 / 24$ & 71 & 709 & 716 & $\mathrm{H} 2+\mathrm{Air}$ & 0.04 & 1450 & 1 & 9.5 & 25 & 801.678 & Circ. & 2.4775 & 0.0026 & 2.4831 & 0.0021 \\
\hline $04 / 24$ & 71 & 719 & 726 & $\mathrm{H} 2+\mathrm{Air}$ & 0.04 & 1450 & 1 & 9.5 & 20 & 801.665 & Circ. & 2.5503 & 0.0025 & 2.5456 & 0.0020 \\
\hline $04 / 24$ & 71 & 729 & 736 & $\mathrm{H} 2+\mathrm{Air}$ & 0.04 & 1450 & 1 & 9.5 & 10 & 801.683 & Circ. & 2.5748 & 0.0019 & 2.5816 & 0.0022 \\
\hline $04 / 24$ & 71 & 739 & 746 & $\mathrm{H} 2+\mathrm{Air}$ & 0.04 & 1450 & 1 & 9.5 & 5 & 801.703 & Circ. & 2.5960 & 0.0019 & 2.6029 & 0.0014 \\
\hline $04 / 24$ & 72 & 749 & 756 & $\mathrm{H} 2+\mathrm{Air}$ & 0.04 & 300 & 1 & 9.5 & 18 & 808.321 & Circ. & 2.5399 & 0.0030 & 2.5625 & 0.0024 \\
\hline $04 / 24$ & 72 & 759 & 766 & $\mathrm{H} 2+\mathrm{Air}$ & 0.04 & 300 & 1 & 9.5 & 10 & 808.252 & Circ. & 2.6027 & 0.0031 & & \\
\hline $04 / 24$ & 72 & 769 & 776 & $\mathrm{H} 2+\mathrm{Air}$ & 0.04 & 300 & 1 & 9.5 & 5 & 808.206 & Circ. & 2.6439 & 0.0018 & & \\
\hline $04 / 27$ & 95 & 1563 & 1568 & $\mathrm{H} 2+\mathrm{Air}$ & 0.05 & 1470 & 1 & 9.5 & 25 & 801.596 & Circ. & 2.2480 & 0.0023 & 2.2624 & 0.0020 \\
\hline $04 / 27$ & 97 & 1543 & 1550 & $\mathrm{H} 2+\mathrm{Air}$ & 0.025 & 1470 & 1 & 9.5 & 25 & 801.596 & Circ. & 2.1810 & 0.0024 & 2.2050 & 0.0022 \\
\hline $04 / 27$ & 98 & 1553 & 1560 & $\mathrm{H} 2+\mathrm{Air}$ & 0.01 & 1470 & 1 & 9.5 & 25 & 801.608 & Circ. & 2.3030 & 0.0023 & 2.3152 & 0.0020 \\
\hline $04 / 27$ & 99 & 1562 & 1570 & $\mathrm{H} 2+\mathrm{Air}$ & 0.002 & 1470 & 1 & 9.5 & 25 & 801.612 & Circ. & 2.3510 & 0.0024 & 2.3722 & 0.0018 \\
\hline $04 / 27$ & 99 & 1573 & 1580 & $\mathrm{H} 2+\mathrm{Air}$ & 0.002 & 1470 & 1 & 9.5 & 20 & 801.621 & Circ. & 2.4592 & 0.0023 & 2.4591 & 0.0019 \\
\hline $04 / 27$ & 99 & 1583 & 1590 & $\mathrm{H} 2+\mathrm{Air}$ & 0.002 & 1470 & 1 & 9.5 & 10 & 801.642 & Circ. & 2.5060 & 0.0018 & 2.5129 & 0.0023 \\
\hline
\end{tabular}




\begin{tabular}{|c|c|c|c|c|c|c|c|c|c|c|c|c|c|c|c|}
\hline Date & Run & $\begin{array}{l}\text { Start } \\
\text { Pulse }\end{array}$ & $\begin{array}{l}\text { End } \\
\text { Pulse }\end{array}$ & $\begin{array}{l}\text { Gas } \\
\text { Species }\end{array}$ & $\begin{array}{l}\text { Gas } \\
\text { Conc. } \\
(\%)\end{array}$ & $\begin{array}{l}\text { Gas } \\
\text { Press. } \\
\text { (psi) }\end{array}$ & $\begin{array}{l}\text { Beam } \\
\text { Int. }\end{array}$ & $\begin{array}{l}\text { Beam } \\
\text { Length } \\
(\mu \mathrm{s})\end{array}$ & $\begin{array}{l}\text { Elec. } \\
\text { Field } \\
(\mathrm{MV} / \mathrm{m})\end{array}$ & $\begin{array}{l}\text { Freq. } \\
(\mathrm{MHz})\end{array}$ & $\begin{array}{l}\text { Circ. } \\
\text { /Hybr. }\end{array}$ & $\mathrm{B} \tau(\mu \mathrm{s})$ & $\begin{array}{l}\text { B } \tau \text { erro } \\
(\mu \mathrm{s})\end{array}$ & $\operatorname{prE} \tau(\mu \mathrm{s})$ & $\begin{array}{l}\mathrm{E} \tau \text { error } \\
(\mu \mathrm{s})\end{array}$ \\
\hline $04 / 27$ & 101 & 1593 & 1600 & $\mathrm{H} 2+\mathrm{Air}$ & 0.002 & 1470 & 1 & 9.5 & 5 & 801.667 & Circ. & 2.5536 & 0.0018 & 2.5647 & 0.0014 \\
\hline $04 / 27$ & 102 & 1603 & 1610 & $\mathrm{H} 2+\mathrm{Air}$ & 0.001 & 1470 & 1 & 9.5 & 25 & 801.606 & Circ. & 2.3536 & 0.0024 & 2.3665 & 0.0019 \\
\hline $04 / 27$ & 102 & 1613 & 1620 & $\mathrm{H} 2+\mathrm{Air}$ & 0.001 & 1470 & 1 & 9.5 & 20 & 801.615 & Circ. & 2.4378 & 0.0023 & 2.4610 & 0.0020 \\
\hline $04 / 27$ & 102 & 1752 & 1760 & $\mathrm{H} 2+\mathrm{Air}$ & 0.001 & 1470 & 1 & 9.5 & 10 & 801.637 & Circ. & 2.5344 & 0.0018 & 2.5395 & 0.0015 \\
\hline $04 / 27$ & 102 & 1763 & 1770 & $\mathrm{H} 2+\mathrm{Air}$ & 0.001 & 1470 & 1 & 9.5 & 5 & 801.662 & Circ. & 2.5598 & 0.0015 & & \\
\hline $05 / 02$ & 119 & 1909 & 1913 & $\mathrm{H} 2+\mathrm{Air}$ & 1 & 1470 & 1 & 9.5 & 50 & 801.476 & Hybr. & 3.8384 & 0.0068 & 3.8760 & 0.0051 \\
\hline $05 / 03$ & 127 & 2255 & 2262 & $\mathrm{H} 2+\mathrm{Air}$ & 1 & 1100 & 1 & 9.5 & 10 & 803.525 & Hybr. & 4.0145 & 0.0146 & 4.0536 & 0.0166 \\
\hline $05 / 03$ & 127 & 2265 & 2272 & $\mathrm{H} 2+\mathrm{Air}$ & 1 & 1100 & 1 & 9.5 & 25 & 803.484 & Hybr. & 3.8682 & 0.0138 & 3.8776 & 0.0155 \\
\hline $05 / 03$ & 127 & 2274 & 2281 & $\mathrm{H} 2+\mathrm{Air}$ & 1 & 1100 & 1 & 9.5 & 40 & 803.421 & Hybr. & 3.4618 & 0.0155 & 3.4705 & 0.0162 \\
\hline $05 / 03$ & 128 & 2284 & 2291 & $\mathrm{H} 2+\mathrm{Air}$ & 0.2 & 1100 & 1 & 9.5 & 10 & 803.687 & Hybr. & 4.4559 & 0.0145 & 4.4291 & 0.0149 \\
\hline $05 / 03$ & 128 & 2296 & 2303 & $\mathrm{H} 2+\mathrm{Air}$ & 0.2 & 1100 & 1 & 9.5 & 25 & 803.687 & Hybr. & 4.4787 & 0.0163 & 4.5123 & 0.0176 \\
\hline $05 / 03$ & 128 & 2306 & 2313 & $\mathrm{H} 2+\mathrm{Air}$ & 0.2 & 1100 & 1 & 9.5 & 40 & 803.656 & Hybr. & 4.4724 & 0.0193 & 4.4653 & 0.0199 \\
\hline $05 / 03$ & 129 & 2316 & 2325 & $\mathrm{H} 2+\mathrm{Air}$ & 0.04 & 1100 & 1 & 9.5 & 40 & 803.711 & Hybr. & 4.3881 & 0.0171 & & \\
\hline $05 / 03$ & 129 & 2329 & 2337 & $\mathrm{H} 2+\mathrm{Air}$ & 0.04 & 1100 & 1 & 9.5 & 25 & 803.594 & Hybr. & 4.2436 & 0.0172 & 4.2194 & 0.0155 \\
\hline $05 / 03$ & 129 & 2341 & 2348 & $\mathrm{H} 2+\mathrm{Air}$ & 0.04 & 1100 & 1 & 9.5 & 10 & 803.722 & Hybr. & 4.2224 & 0.0152 & 4.1738 & 0.0171 \\
\hline $05 / 03$ & 130 & 2754 & 2761 & $\mathrm{H} 2+\mathrm{Air}$ & 0.04 & 1100 & 0.2 & 9.5 & 10 & 803.821 & Hybr. & 3.8794 & 0.0231 & 3.8366 & 0.0233 \\
\hline $05 / 03$ & 130 & 2765 & 2770 & $\mathrm{H} 2+\mathrm{Air}$ & 0.04 & 1100 & 0.2 & 9.5 & 25 & 803.825 & Hybr. & 3.9676 & 0.0255 & & \\
\hline $05 / 03$ & 130 & 2833 & 2840 & $\mathrm{H} 2+\mathrm{Air}$ & 0.04 & 1100 & 0.2 & 9.5 & 40 & 803.804 & Hybr. & 4.1006 & 0.0226 & 4.0777 & 0.0233 \\
\hline $05 / 03$ & 131 & 2843 & 2850 & $\mathrm{H} 2+\mathrm{Air}$ & 0.04 & 800 & 0.2 & 9.5 & 10 & 805.452 & Hybr. & 3.8267 & 0.0178 & 3.7839 & 0.0161 \\
\hline $05 / 03$ & 131 & 2854 & 2861 & $\mathrm{H} 2+\mathrm{Air}$ & 0.04 & 800 & 0.2 & 9.5 & 25 & 805.448 & Hybr. & 3.8391 & 0.0185 & 3.8181 & 0.0170 \\
\hline $05 / 03$ & 131 & 2864 & 2872 & $\mathrm{H} 2+$ Air & 0.04 & 800 & 0.2 & 9.5 & 40 & 805.419 & Hybr. & 3.6069 & 0.0257 & 3.6064 & 0.0229 \\
\hline $05 / 03$ & 132 & 2875 & 2883 & $\mathrm{H} 2+\mathrm{Air}$ & 0.04 & 300 & 0.2 & 9.5 & 10 & 808.441 & Hybr. & 2.2184 & & & \\
\hline $05 / 03$ & 133 & 2884 & 2885 & $\mathrm{H} 2+$ Air & 0.04 & 300 & 0.2 & 9.5 & 10 & 808.441 & Hybr. & 2.2184 & 0.0077 & 2.2136 & 0.0085 \\
\hline $05 / 03$ & 133 & 2886 & 2893 & $\mathrm{H} 2+\mathrm{Air}$ & 0.04 & 300 & 0.2 & 9.5 & 18 & 808.444 & Hybr. & 2.1317 & 0.0075 & 2.1106 & 0.0084 \\
\hline $05 / 03$ & 134 & 2896 & 2904 & $\mathrm{H} 2+\mathrm{Air}$ & 1 & 1470 & 0.2 & 9.5 & 10 & 801.623 & Hybr. & 3.9136 & 0.0086 & 3.8997 & 0.0076 \\
\hline $05 / 03$ & 134 & 2914 & 2921 & $\mathrm{H} 2+\mathrm{Air}$ & 1 & 1470 & 0.2 & 9.5 & 25 & 801.623 & Hybr. & 3.9112 & 0.0099 & 3.9003 & 0.0087 \\
\hline 05/03 & 134 & 2924 & 2931 & $\mathrm{H} 2+\mathrm{Air}$ & 1 & 1470 & 0.2 & 9.5 & 40 & 801.619 & Hybr. & 3.9644 & 0.0106 & 3.9355 & 0.0090 \\
\hline $05 / 03$ & 135 & 2934 & 2941 & $\mathrm{H} 2+\mathrm{Air}$ & 1 & 1100 & 0.2 & 9.5 & 10 & 803.614 & Hybr. & 4.4198 & 0.0150 & 4.4052 & 0.0161 \\
\hline $05 / 03$ & 135 & 2944 & 2951 & $\mathrm{H} 2+\mathrm{Air}$ & 1 & 1100 & 0.2 & 9.5 & 25 & 803.615 & Hybr. & 4.4379 & 0.0185 & 4.4498 & 0.0204 \\
\hline $05 / 03$ & 135 & 2954 & 2961 & $\mathrm{H} 2+\mathrm{Air}$ & 1 & 1100 & 0.2 & 9.5 & 40 & 803.595 & Hybr. & 4.3595 & 0.0191 & 4.3817 & 0.0215 \\
\hline $05 / 03$ & 136 & 2964 & 2971 & $\mathrm{H} 2+\mathrm{Air}$ & 1 & 800 & 0.2 & 9.5 & 10 & 805.378 & Hybr. & 3.4776 & 0.0212 & 3.4746 & 0.0193 \\
\hline $05 / 03$ & 136 & 2974 & 2981 & $\mathrm{H} 2+\mathrm{Air}$ & 1 & 800 & 0.2 & 9.5 & 25 & 805.376 & Hybr. & 3.3122 & 0.0258 & 3.3155 & 0.0233 \\
\hline $05 / 03$ & 136 & 2984 & 2991 & $\mathrm{H} 2+\mathrm{Air}$ & 1 & 800 & 0.2 & 9.5 & 40 & 805.341 & Hybr. & 2.8525 & 0.0283 & 2.8632 & 0.0267 \\
\hline $05 / 03$ & 137 & 2994 & 3001 & $\mathrm{H} 2+\mathrm{Air}$ & 1 & 300 & 0.2 & 9.5 & 10 & 808.398 & Hybr. & 1.6749 & 0.0057 & 1.6530 & 0.0056 \\
\hline $05 / 03$ & 137 & 3004 & 3011 & $\mathrm{H} 2+\mathrm{Air}$ & 1 & 300 & 0.2 & 9.5 & 18 & 808.396 & Hybr. & 1.6088 & 0.0059 & 1.5862 & 0.0059 \\
\hline $05 / 03$ & 138 & 3014 & 3021 & $\mathrm{H} 2+\mathrm{Air}$ & 0.2 & 1470 & 0.2 & 9.5 & 10 & 801.672 & Hybr. & 3.7398 & 0.0087 & 3.7445 & 0.0076 \\
\hline $05 / 03$ & 138 & 3023 & 3030 & $\mathrm{H} 2+\mathrm{Air}$ & 0.2 & 1470 & 0.2 & 9.5 & 25 & 801.683 & Hybr. & 3.8023 & 0.0098 & 3.7826 & 0.0088 \\
\hline $05 / 03$ & 138 & 3033 & 3040 & $\mathrm{H} 2+\mathrm{Air}$ & 0.2 & 1470 & 0.2 & 9.5 & 40 & 801.664 & Hybr. & 3.8689 & 0.0104 & 3.8371 & 0.0090 \\
\hline $05 / 03$ & 139 & 3043 & 3050 & $\mathrm{H} 2+\mathrm{Air}$ & 0.2 & 1100 & 0.2 & 9.5 & 10 & 803.697 & Hybr. & 4.3844 & 0.0131 & 4.3590 & 0.0140 \\
\hline $05 / 03$ & 139 & 3053 & 3062 & $\mathrm{H} 2+\mathrm{Air}$ & 0.2 & 1100 & 0.2 & 9.5 & 25 & 803.698 & Hybr. & 4.4144 & 0.0150 & 4.3981 & 0.0165 \\
\hline
\end{tabular}




\begin{tabular}{|c|c|c|c|c|c|c|c|c|c|c|c|c|c|c|c|}
\hline Date & Run & $\begin{array}{l}\text { Start } \\
\text { Pulse }\end{array}$ & $\begin{array}{l}\text { End } \\
\text { Pulse }\end{array}$ & $\begin{array}{l}\text { Gas } \\
\text { Species }\end{array}$ & $\begin{array}{l}\text { Gas } \\
\text { Conc. } \\
(\%)\end{array}$ & $\begin{array}{l}\text { Gas } \\
\text { Press. } \\
\text { (psi) }\end{array}$ & $\begin{array}{l}\text { Beam } \\
\text { Int. }\end{array}$ & $\begin{array}{l}\text { Beam } \\
\text { Length } \\
(\mu \mathrm{s})\end{array}$ & $\begin{array}{l}\text { Elec. } \\
\text { Field } \\
(\mathrm{MV} / \mathrm{m})\end{array}$ & $\begin{array}{l}\text { Freq. } \\
(\mathrm{MHz})\end{array}$ & $\begin{array}{l}\text { Circ. } \\
\text { /Hybr. }\end{array}$ & $\mathrm{B} \tau(\mu \mathrm{s})$ & $\begin{array}{l}\text { B } \tau \text { erro } \\
(\mu \mathrm{s})\end{array}$ & $\mathrm{rE} \tau(\mu \mathrm{s})$ & $\begin{array}{l}\text { E } \tau \text { error } \\
(\mu \mathrm{s})\end{array}$ \\
\hline $05 / 03$ & 139 & 3065 & 3072 & $\mathrm{H} 2+\mathrm{Air}$ & 0.2 & 1100 & 0.2 & 9.5 & 40 & 803.68 & Hybr. & 4.4323 & 0.0185 & 4.4070 & 0.0194 \\
\hline $05 / 03$ & 141 & 3077 & 3084 & $\mathrm{H} 2+\mathrm{Air}$ & 0.2 & 800 & 0.2 & 9.5 & 10 & 805.432 & Hybr. & 3.6391 & 0.0193 & 3.6205 & 0.0179 \\
\hline $05 / 03$ & 141 & 3088 & 3095 & $\mathrm{H} 2+\mathrm{Air}$ & 0.2 & 800 & 0.2 & 9.5 & 20 & 805.43 & Hybr. & 3.6253 & 0.0201 & 3.5946 & 0.0193 \\
\hline $05 / 03$ & 142 & 3116 & 3123 & $\mathrm{H} 2+\mathrm{Air}$ & 0.2 & 800 & 0.2 & 9.5 & 25 & 805.421 & Hybr. & 3.5215 & 0.0230 & 3.5171 & 0.0208 \\
\hline $05 / 03$ & 143 & 3127 & 3134 & $\mathrm{H} 2+\mathrm{Air}$ & 0.2 & 300 & 0.2 & 9.5 & 10 & 808.433 & Hybr. & 1.7141 & 0.0056 & 1.6902 & 0.0057 \\
\hline $05 / 03$ & 143 & 3137 & 3146 & $\mathrm{H} 2+\mathrm{Air}$ & 0.2 & 300 & 0.2 & 9.5 & 18 & 808.422 & Hybr. & 1.6279 & 0.0058 & 1.6030 & 0.0058 \\
\hline $05 / 03$ & 144 & 3150 & 3157 & $\mathrm{H} 2+\mathrm{Air}$ & 0.022 & 1385 & 0.2 & 9.5 & 10 & 802.158 & Hybr. & 1.7460 & 0.0063 & 1.7165 & 0.0064 \\
\hline $05 / 03$ & 144 & 3160 & 3168 & $\mathrm{H} 2+\mathrm{Air}$ & 0.022 & 1385 & 0.2 & 9.5 & 25 & 802.163 & Hybr. & 1.6833 & 0.0060 & 1.6651 & 0.0059 \\
\hline $05 / 03$ & 144 & 3172 & 3180 & $\mathrm{H} 2+\mathrm{Air}$ & 0.022 & 1385 & 0.2 & 9.5 & 40 & 802.147 & Hybr. & 1.4835 & 0.0084 & 1.4713 & 0.0083 \\
\hline $05 / 03$ & 144 & 3183 & 3190 & $\mathrm{H} 2+\mathrm{Air}$ & 0.022 & 1385 & 0.2 & 9.5 & 50 & 802.128 & Hybr. & 1.4312 & 0.0103 & 1.4121 & 0.0100 \\
\hline
\end{tabular}


APPENDIX D

ENERGY LOSS DATA 
Table D.1. $d w$ for pure hydrogen.

\begin{tabular}{|c|c|c|c|c|}
\hline $\begin{array}{l}X \\
(\mathrm{MV} / \mathrm{m} / \mathrm{psi})\end{array}$ & $\begin{array}{l}X \quad \text { error } \\
(\mathrm{MV} / \mathrm{m} / \mathrm{psi})\end{array}$ & $\begin{array}{l}\text { Pressure } \\
\text { (psi) }\end{array}$ & $\begin{array}{l}d w \\
(\mathrm{~J} / \mathrm{cycle} / \mathrm{pa}\end{array}$ & $\begin{array}{ll}d w & \text { error } \\
r(\mathrm{~J} / \text { cycle/pair })\end{array}$ \\
\hline 0.0140 & 0.0004298 & 300 & $6.013 \mathrm{E}-18$ & $2.623 \mathrm{E}-19$ \\
\hline 0.0289 & 0.0005840 & 300 & $2.101 \mathrm{E}-17$ & $1.007 \mathrm{E}-18$ \\
\hline 0.0531 & 0.0005792 & 300 & $5.694 \mathrm{E}-17$ & $1.885 \mathrm{E}-18$ \\
\hline 0.0085 & 0.0002402 & 500 & $4.951 \mathrm{E}-18$ & $2.391 \mathrm{E}-19$ \\
\hline 0.0175 & 0.0002130 & 500 & $1.551 \mathrm{E}-17$ & $5.973 \mathrm{E}-19$ \\
\hline 0.0351 & 0.0001899 & 500 & $4.754 \mathrm{E}-17$ & $1.819 \mathrm{E}-18$ \\
\hline 0.0441 & 0.0007558 & 500 & $7.039 \mathrm{E}-17$ & $2.035 \mathrm{E}-18$ \\
\hline 0.0048 & 0.0000993 & 800 & $3.184 \mathrm{E}-18$ & $2.874 \mathrm{E}-19$ \\
\hline 0.0100 & 0.0003779 & 800 & $1.039 \mathrm{E}-17$ & $5.297 \mathrm{E}-19$ \\
\hline 0.0200 & 0.0002165 & 800 & $3.093 \mathrm{E}-17$ & $1.695 \mathrm{E}-18$ \\
\hline 0.0253 & 0.0001604 & 800 & $4.547 \mathrm{E}-17$ & $2.161 \mathrm{E}-18$ \\
\hline 0.0043 & 0.0001327 & 950 & $2.818 \mathrm{E}-18$ & $1.896 \mathrm{E}-19$ \\
\hline 0.0089 & 0.0002832 & 950 & $8.804 \mathrm{E}-18$ & $3.409 \mathrm{E}-19$ \\
\hline 0.0182 & 0.0004672 & 950 & $2.815 \mathrm{E}-17$ & $1.159 \mathrm{E}-18$ \\
\hline 0.0221 & 0.0012667 & 950 & $3.959 \mathrm{E}-17$ & $2.413 \mathrm{E}-18$ \\
\hline 0.0266 & 0.0011435 & 950 & $5.132 \mathrm{E}-17$ & $2.346 \mathrm{E}-18$ \\
\hline 0.0350 & 0.0010116 & 950 & $9.126 \mathrm{E}-17$ & $2.813 \mathrm{E}-18$ \\
\hline 0.0038 & 0.0001044 & 1100 & $2.730 \mathrm{E}-18$ & $1.666 \mathrm{E}-19$ \\
\hline 0.0073 & 0.0002971 & 1100 & $6.423 \mathrm{E}-18$ & $1.968 \mathrm{E}-19$ \\
\hline 0.0151 & 0.0004336 & 1100 & $2.382 \mathrm{E}-17$ & $1.143 \mathrm{E}-18$ \\
\hline 0.0190 & 0.0009207 & 1100 & $3.277 \mathrm{E}-17$ & $1.268 \mathrm{E}-18$ \\
\hline 0.0242 & 0.0004574 & 1100 & $5.311 \mathrm{E}-17$ & $1.735 \mathrm{E}-18$ \\
\hline 0.0319 & 0.0004295 & 1100 & $8.646 \mathrm{E}-17$ & $1.821 \mathrm{E}-18$ \\
\hline 0.0036 & 0.0000713 & 1140 & $2.701 \mathrm{E}-18$ & $4.866 \mathrm{E}-20$ \\
\hline 0.0069 & 0.0001847 & 1140 & $6.191 \mathrm{E}-18$ & $1.754 \mathrm{E}-19$ \\
\hline 0.0141 & 0.0002754 & 1140 & $2.224 \mathrm{E}-17$ & $5.075 \mathrm{E}-19$ \\
\hline 0.0189 & 0.0003325 & 1140 & $3.132 \mathrm{E}-17$ & $6.699 \mathrm{E}-19$ \\
\hline 0.0031 & 0.0000504 & 1300 & $1.923 \mathrm{E}-18$ & $9.320 \mathrm{E}-20$ \\
\hline 0.0064 & 0.0000773 & 1300 & $6.593 \mathrm{E}-18$ & $2.731 \mathrm{E}-19$ \\
\hline 0.0129 & 0.0001228 & 1300 & $2.142 \mathrm{E}-17$ & $9.953 \mathrm{E}-19$ \\
\hline 0.0159 & 0.0001684 & 1300 & $3.012 \mathrm{E}-17$ & $1.354 \mathrm{E}-18$ \\
\hline 0.0026 & 0.0000368 & 1470 & $1.522 \mathrm{E}-18$ & $6.552 \mathrm{E}-20$ \\
\hline 0.0051 & 0.0001082 & 1470 & $4.904 \mathrm{E}-18$ & $3.198 \mathrm{E}-19$ \\
\hline 0.0102 & 0.0002277 & 1470 & $1.626 \mathrm{E}-17$ & $8.633 \mathrm{E}-19$ \\
\hline 0.0135 & 0.0000857 & 1470 & $2.502 \mathrm{E}-17$ & $7.934 \mathrm{E}-19$ \\
\hline 0.0214 & 0.0002969 & 1470 & $5.399 \mathrm{E}-17$ & $1.855 \mathrm{E}-18$ \\
\hline 0.0251 & 0.0003269 & 1470 & $7.375 \mathrm{E}-17$ & $2.979 \mathrm{E}-18$ \\
\hline 0.0027 & 0.0000393 & 1520 & $1.653 \mathrm{E}-18$ & $8.004 \mathrm{E}-20$ \\
\hline 0.0054 & 0.0001209 & 1520 & $5.731 \mathrm{E}-18$ & $1.729 \mathrm{E}-19$ \\
\hline
\end{tabular}




\begin{tabular}{|c|c|c|c|c|}
\hline $\begin{array}{l}X \\
(\mathrm{MV} / \mathrm{m} / \mathrm{psi})\end{array}$ & $\begin{array}{l}X \quad \text { erro } \\
(\mathrm{MV} / \mathrm{m} / \mathrm{psi})\end{array}$ & $\begin{array}{l}\text { Pressure } \\
\text { (psi) }\end{array}$ & $\begin{array}{l}d w \\
(\mathrm{~J} / \text { cycle } / \mathrm{p}\end{array}$ & $\begin{array}{cc}d w & \text { error } \\
\text { r }(\mathrm{J} / \text { cycle/pair })\end{array}$ \\
\hline 0.0109 & 0.0001039 & 1520 & $1.807 \mathrm{E}-17$ & $7.043 \mathrm{E}-19$ \\
\hline 0.0132 & 0.0006130 & 1520 & $2.657 \mathrm{E}-17$ & $2.202 \mathrm{E}-18$ \\
\hline
\end{tabular}

Table D.2. $d w$ for dry air-doped hydrogen

\begin{tabular}{|c|c|c|c|c|c|}
\hline $\begin{array}{l}X \\
(\mathrm{MV} / \mathrm{m} / \mathrm{psi})\end{array}$ & $\begin{array}{l}X \quad \text { error } \\
(\mathrm{MV} / \mathrm{m} / \mathrm{psi})\end{array}$ & $\begin{array}{l}\text { Pressure } \\
\text { (psi) }\end{array}$ & $\begin{array}{l}\text { Concentration } \\
(\%)\end{array}$ & $\begin{array}{l}d w \\
(\mathrm{~J} / \text { cycle/pair }\end{array}$ & $\begin{array}{ll}d w & \text { error } \\
\mathrm{r}(\mathrm{J} / \mathrm{cycle} / \text { pair })\end{array}$ \\
\hline 0.01386 & 0.0001042 & 300 & 0.04 & $4.8387 \mathrm{E}-18$ & $7.9822 \mathrm{E}-20$ \\
\hline 0.02770 & 0.0012482 & 300 & 0.04 & $1.4135 \mathrm{E}-17$ & $1.2650 \mathrm{E}-18$ \\
\hline 0.04975 & 0.0020516 & 300 & 0.04 & $4.3012 \mathrm{E}-17$ & $4.4728 \mathrm{E}-18$ \\
\hline 0.01380 & 0.0001336 & 300 & 0.2 & $1.8251 \mathrm{E}-18$ & $2.2727 \mathrm{E}-19$ \\
\hline 0.02847 & 0.0006371 & 300 & 0.2 & $8.9650 \mathrm{E}-18$ & $1.0188 \mathrm{E}-18$ \\
\hline 0.04710 & 0.0058567 & 300 & 0.2 & $3.0479 \mathrm{E}-17$ & $1.2195 \mathrm{E}-17$ \\
\hline 0.01418 & 0.0000326 & 300 & 1 & $5.0447 \mathrm{E}-19$ & $4.1981 \mathrm{E}-20$ \\
\hline 0.02869 & 0.0001598 & 300 & 1 & $2.6359 \mathrm{E}-18$ & $8.6544 \mathrm{E}-19$ \\
\hline 0.05286 & 0.0003550 & 300 & 1 & $1.3916 \mathrm{E}-17$ & $5.5100 \mathrm{E}-18$ \\
\hline 0.01391 & 0.0000378 & 300 & 5 & $1.4605 \mathrm{E}-19$ & $1.3271 \mathrm{E}-20$ \\
\hline 0.02852 & 0.0000699 & 300 & 5 & $8.1802 \mathrm{E}-19$ & $4.0377 \mathrm{E}-20$ \\
\hline 0.05257 & 0.0001874 & 300 & 5 & $3.5854 \mathrm{E}-18$ & $1.5049 \mathrm{E}-19$ \\
\hline 0.00564 & 0.0001174 & 735 & 10 & $2.5707 \mathrm{E}-20$ & $1.2914 \mathrm{E}-20$ \\
\hline 0.01128 & 0.0001947 & 735 & 10 & $1.1119 \mathrm{E}-19$ & $1.6444 \mathrm{E}-20$ \\
\hline 0.02170 & 0.0003892 & 735 & 10 & $3.9066 \mathrm{E}-19$ & $4.4083 \mathrm{E}-20$ \\
\hline 0.02860 & 0.0001394 & 735 & 10 & $7.3497 \mathrm{E}-19$ & $1.1025 \mathrm{E}-19$ \\
\hline 0.01023 & 0.0000626 & 800 & 0.04 & 4.7293E-19 & $2.0240 \mathrm{E}-19$ \\
\hline 0.02574 & 0.0003054 & 800 & 0.04 & $3.6069 \mathrm{E}-18$ & $7.4795 \mathrm{E}-19$ \\
\hline 0.04204 & 0.0006037 & 800 & 0.04 & $1.4422 \mathrm{E}-17$ & $4.1365 \mathrm{E}-18$ \\
\hline 0.01013 & 0.0001035 & 800 & 0.2 & $2.5237 \mathrm{E}-19$ & $6.2604 \mathrm{E}-20$ \\
\hline 0.02081 & 0.0000524 & 800 & 0.2 & $1.2223 \mathrm{E}-18$ & $9.5714 \mathrm{E}-20$ \\
\hline 0.02589 & 0.0000378 & 800 & 0.2 & $2.3511 \mathrm{E}-18$ & $1.1422 \mathrm{E}-19$ \\
\hline 0.01006 & 0.0000575 & 800 & 1 & $1.6573 \mathrm{E}-19$ & $2.6105 \mathrm{E}-20$ \\
\hline 0.02526 & 0.0002354 & 800 & 1 & $1.3738 \mathrm{E}-18$ & $1.0132 \mathrm{E}-19$ \\
\hline 0.04127 & 0.0005075 & 800 & 1 & $4.2816 \mathrm{E}-18$ & $2.0855 \mathrm{E}-19$ \\
\hline 0.00752 & 0.0000289 & 1100 & 0.04 & $1.3711 \mathrm{E}-19$ & $1.6406 \mathrm{E}-20$ \\
\hline 0.01845 & 0.0000235 & 1100 & 0.04 & $1.2248 \mathrm{E}-18$ & $1.6349 \mathrm{E}-19$ \\
\hline 0.02960 & 0.0004132 & 1100 & 0.04 & $3.8556 \mathrm{E}-18$ & $1.4988 \mathrm{E}-19$ \\
\hline 0.00767 & 0.0000102 & 1100 & 0.2 & $9.4938 \mathrm{E}-20$ & $9.0626 \mathrm{E}-21$ \\
\hline 0.01893 & 0.0000735 & 1100 & 0.2 & $7.7910 \mathrm{E}-19$ & $8.3783 \mathrm{E}-20$ \\
\hline
\end{tabular}




\begin{tabular}{|c|c|c|c|c|c|}
\hline $\begin{array}{l}X \\
(\mathrm{MV} / \mathrm{m} / \mathrm{psi})\end{array}$ & $\begin{array}{l}X \quad \text { error } \\
(\mathrm{MV} / \mathrm{m} / \mathrm{psi})\end{array}$ & $\begin{array}{l}\text { Pressure } \\
(\mathrm{psi})\end{array}$ & $\begin{array}{l}\text { Concentration } \\
(\%)\end{array}$ & $\begin{array}{l}d w \\
(\mathrm{~J} / \text { cycle/pair }\end{array}$ & $\begin{array}{lc}d w & \text { error } \\
\mathrm{r}(\mathrm{J} / \text { cycle/pair })\end{array}$ \\
\hline 0.03019 & 0.0000914 & 1100 & 0.2 & $2.4568 \mathrm{E}-18$ & $3.2295 \mathrm{E}-19$ \\
\hline 0.00381 & 0.0000390 & 1100 & 1 & $2.7387 \mathrm{E}-20$ & $3.7259 \mathrm{E}-21$ \\
\hline 0.00777 & 0.0000752 & 1100 & 1 & $9.8127 \mathrm{E}-20$ & $2.2685 \mathrm{E}-20$ \\
\hline 0.01560 & 0.0000882 & 1100 & 1 & $5.0987 \mathrm{E}-19$ & $2.2027 \mathrm{E}-20$ \\
\hline 0.01969 & 0.0002665 & 1100 & 1 & $8.2611 \mathrm{E}-19$ & $1.7694 \mathrm{E}-19$ \\
\hline 0.03048 & 0.0003091 & 1100 & 1 & $1.9056 \mathrm{E}-18$ & $1.0341 \mathrm{E}-19$ \\
\hline 0.00346 & 0.0000139 & 1100 & 5 & $1.3002 \mathrm{E}-20$ & $1.8448 \mathrm{E}-21$ \\
\hline 0.00708 & 0.0000733 & 1100 & 5 & $6.3776 \mathrm{E}-20$ & $3.1276 \mathrm{E}-21$ \\
\hline 0.01505 & 0.0001228 & 1100 & 5 & $3.2808 \mathrm{E}-19$ & $1.5690 \mathrm{E}-20$ \\
\hline 0.01832 & 0.0000530 & 1100 & 5 & $5.2364 \mathrm{E}-19$ & $2.4126 \mathrm{E}-20$ \\
\hline 0.00336 & 0.0001082 & 1100 & 6.78 & $1.1507 \mathrm{E}-20$ & $1.7432 \mathrm{E}-21$ \\
\hline 0.00693 & 0.0001630 & 1100 & 6.78 & $5.5797 \mathrm{E}-20$ & $5.2563 \mathrm{E}-21$ \\
\hline 0.01388 & 0.0003595 & 1100 & 6.78 & $2.6325 \mathrm{E}-19$ & $1.9883 \mathrm{E}-20$ \\
\hline 0.01832 & 0.0000295 & 1100 & 6.78 & $4.6554 \mathrm{E}-19$ & $2.0467 \mathrm{E}-20$ \\
\hline 0.00599 & 0.0000666 & 1385 & 0.022 & $1.4957 \mathrm{E}-19$ & $7.5997 \mathrm{E}-21$ \\
\hline 0.01539 & 0.0000617 & 1385 & 0.022 & $1.1973 \mathrm{E}-18$ & $5.1791 \mathrm{E}-20$ \\
\hline 0.02475 & 0.0002061 & 1385 & 0.022 & $3.9382 \mathrm{E}-18$ & $2.2993 \mathrm{E}-19$ \\
\hline 0.02708 & 0.0000963 & 1385 & 0.022 & $4.8833 \mathrm{E}-18$ & $1.7928 \mathrm{E}-19$ \\
\hline 0.00265 & 0.0000394 & 1450 & 0.04 & $3.0871 \mathrm{E}-20$ & $3.2481 \mathrm{E}-21$ \\
\hline 0.00519 & 0.0000889 & 1450 & 0.04 & $1.3588 \mathrm{E}-19$ & $1.6823 \mathrm{E}-20$ \\
\hline 0.01390 & 0.0000524 & 1450 & 0.04 & $1.1986 \mathrm{E}-18$ & $8.3971 \mathrm{E}-20$ \\
\hline 0.00257 & 0.0000662 & 1450 & 0.2 & $1.6820 \mathrm{E}-20$ & $1.2092 \mathrm{E}-21$ \\
\hline 0.00519 & 0.0001191 & 1450 & 0.2 & $7.8623 \mathrm{E}-20$ & $8.2138 \mathrm{E}-21$ \\
\hline 0.01380 & 0.0000660 & 1450 & 0.2 & $6.5397 \mathrm{E}-19$ & $4.0135 \mathrm{E}-20$ \\
\hline 0.00284 & 0.0000345 & 1450 & 5 & $8.9036 \mathrm{E}-21$ & $8.0315 \mathrm{E}-22$ \\
\hline 0.00551 & 0.0001037 & 1450 & 5 & $3.8715 \mathrm{E}-20$ & $3.2321 \mathrm{E}-21$ \\
\hline 0.01460 & 0.0000691 & 1450 & 5 & $3.5621 \mathrm{E}-19$ & $1.1882 \mathrm{E}-20$ \\
\hline 0.00252 & 0.0000583 & 1470 & 0.001 & $2.7226 \mathrm{E}-19$ & $4.3519 \mathrm{E}-20$ \\
\hline 0.00478 & 0.0001023 & 1470 & 0.001 & $9.6851 \mathrm{E}-19$ & $2.0097 \mathrm{E}-19$ \\
\hline 0.01040 & 0.0001922 & 1470 & 0.001 & $5.4125 \mathrm{E}-18$ & $7.8034 \mathrm{E}-19$ \\
\hline 0.01323 & 0.0001023 & 1470 & 0.001 & $8.7037 \mathrm{E}-18$ & $5.8606 \mathrm{E}-19$ \\
\hline 0.00259 & 0.0000405 & 1470 & 0.002 & $1.3494 \mathrm{E}-19$ & $1.2469 \mathrm{E}-20$ \\
\hline 0.00503 & 0.0001237 & 1470 & 0.002 & $5.2968 \mathrm{E}-19$ & $6.7224 \mathrm{E}-20$ \\
\hline 0.01064 & & 1470 & 0.002 & $3.1157 \mathrm{E}-18$ & $2.4734 \mathrm{E}-19$ \\
\hline 0.01334 & 0.0001217 & 1470 & 0.002 & $5.7536 \mathrm{E}-18$ & $5.7191 \mathrm{E}-19$ \\
\hline 0.01362 & 0.0000847 & 1470 & 0.01 & $1.3716 \mathrm{E}-17$ & $2.1699 \mathrm{E}-17$ \\
\hline 0.01337 & 0.0000871 & 1470 & 0.025 & $6.5334 \mathrm{E}-19$ & $9.4445 \mathrm{E}-20$ \\
\hline 0.01339 & 0.0001093 & 1470 & 0.05 & $6.3514 \mathrm{E}-19$ & $4.1834 \mathrm{E}-20$ \\
\hline 0.00539 & 0.0000133 & 1470 & 0.2 & $5.6440 \mathrm{E}-20$ & $2.3717 \mathrm{E}-21$ \\
\hline 0.01334 & 0.0000405 & 1470 & 0.2 & $4.0819 \mathrm{E}-19$ & $4.0131 \mathrm{E}-20$ \\
\hline 0.02095 & 0.0001756 & 1470 & 0.2 & $1.2385 \mathrm{E}-18$ & $8.7556 \mathrm{E}-20$ \\
\hline
\end{tabular}




\begin{tabular}{|c|c|c|c|c|c|}
\hline $\begin{array}{l}X \\
(\mathrm{MV} / \mathrm{m} / \mathrm{psi})\end{array}$ & $\begin{array}{l}X \quad \text { error } \\
(\mathrm{MV} / \mathrm{m} / \mathrm{psi})\end{array}$ & $\begin{array}{l}\text { rPressure } \\
\text { (psi) }\end{array}$ & $\begin{array}{l}\text { Concentration } \\
(\%)\end{array}$ & $\begin{array}{l}d w \\
(\mathrm{~J} / \text { cycle/pair }\end{array}$ & $\begin{array}{lc}d w & \text { error } \\
r(\mathrm{~J} / \text { cycle/pair })\end{array}$ \\
\hline 0.00272 & 0.0000215 & 1470 & 1 & $1.4404 \mathrm{E}-20$ & $1.4090 \mathrm{E}-21$ \\
\hline 0.00534 & 0.0000346 & 1470 & 1 & $5.2858 \mathrm{E}-20$ & $9.2725 \mathrm{E}-21$ \\
\hline 0.01099 & 0.0000551 & 1470 & 1 & $3.0852 \mathrm{E}-19$ & $1.1807 \mathrm{E}-20$ \\
\hline 0.01351 & 0.0002681 & 1470 & 1 & $4.4404 \mathrm{E}-19$ & $1.1629 \mathrm{E}-19$ \\
\hline 0.02123 & 0.0001130 & 1470 & 1 & $1.0601 \mathrm{E}-18$ & $4.4116 \mathrm{E}-20$ \\
\hline
\end{tabular}


APPENDIX E

ELECTRON-ION RECOMBINATION DATA 
Table E.1. $\beta$ for low beam intensity hydrogen, calculated at equilibrium

\begin{tabular}{|c|c|c|c|c|c|c|c|c|}
\hline $\begin{array}{l}E_{0} \\
(\mathrm{MV} / \mathrm{m})\end{array}$ & $\begin{array}{l}E_{0} \text { error } \\
(\mathrm{MV} / \mathrm{m})\end{array}$ & $\begin{array}{l}E_{\text {equil }} \\
(\mathrm{MV} / \mathrm{m})\end{array}$ & $\begin{array}{l}E_{\text {equil }} \\
\text { error } \\
(\mathrm{MV} / \mathrm{m})\end{array}$ & $\begin{array}{l}\text { Pressure } \\
(\mathrm{psi})\end{array}$ & $\begin{array}{l}\text { Beam Inten- } \\
\text { sity (pro- } \\
\text { tons/pulse) }\end{array}$ & $\begin{array}{l}\text { Beam Intensity } \\
\text { error (pro- } \\
\text { tons/pulse) }\end{array}$ & $\beta\left(\frac{\mathrm{cm}^{3}}{\mathrm{~s}}\right)$ & $\begin{array}{l}\beta \quad \text { error } \\
\left(\frac{\mathrm{cm}^{3}}{\mathrm{~s}}\right)\end{array}$ \\
\hline 4.426 & 0.032 & 0.235 & 0.026 & 300 & $5.8737 \mathrm{E}+9$ & $1.4236 \mathrm{E}+9$ & $4.9586 \mathrm{E}-7$ & $1.1160 \mathrm{E}-7$ \\
\hline 7.460 & 2.242 & 0.478 & 0.049 & 300 & $4.2227 \mathrm{E}+9$ & $2.1778 \mathrm{E}+9$ & $3.4702 \mathrm{E}-7$ & $6.1095 \mathrm{E}-8$ \\
\hline 16.535 & 0.186 & 1.845 & 0.122 & 300 & $1.7948 \mathrm{E}+9$ & $3.0829 \mathrm{E}+8$ & $2.2669 \mathrm{E}-7$ & $3.0811 \mathrm{E}-8$ \\
\hline 4.414 & 0.131 & 0.793 & 0.105 & 500 & $3.6419 \mathrm{E}+9$ & $2.4995 \mathrm{E}+9$ & $1.9334 \mathrm{E}-6$ & $1.1636 \mathrm{E}-6$ \\
\hline 8.950 & 0.806 & 1.550 & 0.182 & 500 & $3.7215 \mathrm{E}+9$ & $2.5643 \mathrm{E}+9$ & $1.2755 \mathrm{E}-6$ & $1.0345 \mathrm{E}-6$ \\
\hline 4.030 & 0.082 & 0.703 & 0.062 & 800 & $5.4209 \mathrm{E}+9$ & $1.4033 \mathrm{E}+9$ & $1.4428 \mathrm{E}-6$ & $4.1080 \mathrm{E}-7$ \\
\hline 6.739 & 2.033 & 1.412 & 0.278 & 800 & $3.3642 \mathrm{E}+9$ & $2.0368 \mathrm{E}+9$ & $1.5067 \mathrm{E}-6$ & $1.3048 \mathrm{E}-6$ \\
\hline 15.747 & 5.750 & 3.442 & 0.644 & 800 & $3.3590 \mathrm{E}+9$ & $2.2425 \mathrm{E}+9$ & $1.1575 \mathrm{E}-6$ & $1.6926 \mathrm{E}-6$ \\
\hline 21.211 & 0.045 & 6.850 & 0.058 & 800 & $1.4283 \mathrm{E}+9$ & $2.4080 \mathrm{E}+8$ & $5.4847 \mathrm{E}-7$ & $7.2286 \mathrm{E}-8$ \\
\hline 4.292 & 0.174 & 0.815 & 0.109 & 950 & $5.0074 \mathrm{E}+9$ & $1.1140 \mathrm{E}+9$ & $2.1667 \mathrm{E}-6$ & $7.5412 \mathrm{E}-7$ \\
\hline 7.301 & 2.167 & 1.689 & 0.184 & 950 & $3.7304 \mathrm{E}+9$ & $1.7920 \mathrm{E}+9$ & $1.6939 \mathrm{E}-6$ & $9.4829 \mathrm{E}-7$ \\
\hline 17.903 & 5.009 & 3.647 & 0.445 & 950 & $4.3081 \mathrm{E}+9$ & $1.7102 \mathrm{E}+9$ & $7.8279 \mathrm{E}-7$ & $5.2921 \mathrm{E}-7$ \\
\hline 17.398 & 5.120 & 4.667 & 0.799 & 1100 & $3.7258 \mathrm{E}+9$ & $1.5118 \mathrm{E}+9$ & $1.2736 \mathrm{E}-6$ & $1.6956 \mathrm{E}-6$ \\
\hline 20.507 & 2.765 & 8.596 & 1.084 & 1100 & $1.7858 \mathrm{E}+9$ & $3.6608 \mathrm{E}+8$ & $1.1374 \mathrm{E}-6$ & $4.9462 \mathrm{E}-7$ \\
\hline 37.034 & 0.193 & 13.880 & 0.292 & 1100 & $1.0439 \mathrm{E}+9$ & $1.2840 \mathrm{E}+8$ & $1.3863 \mathrm{E}-6$ & $3.4041 \mathrm{E}-7$ \\
\hline 4.172 & 0.072 & 2.245 & 0.214 & 1300 & $3.9934 \mathrm{E}+9$ & $2.0826 \mathrm{E}+9$ & $1.8981 \mathrm{E}-5$ & $2.4345 \mathrm{E}-5$ \\
\hline 8.606 & 0.072 & 4.479 & 0.388 & 1300 & $4.3691 \mathrm{E}+9$ & $2.2748 \mathrm{E}+9$ & $8.8160 \mathrm{E}-6$ & $9.0296 \mathrm{E}-6$ \\
\hline 18.529 & 1.737 & 8.667 & 0.693 & 1300 & $5.5070 \mathrm{E}+9$ & $2.6529 \mathrm{E}+9$ & $3.8390 \mathrm{E}-6$ & $4.0150 \mathrm{E}-6$ \\
\hline 21.239 & 1.445 & 11.005 & 0.647 & 1300 & $2.9082 \mathrm{E}+9$ & $1.3119 \mathrm{E}+9$ & $2.9839 \mathrm{E}-6$ & $3.9163 \mathrm{E}-6$ \\
\hline 35.493 & 2.979 & 21.553 & 3.371 & 1470 & $1.3591 \mathrm{E}+9$ & $1.6398 \mathrm{E}+9$ & $5.1431 \mathrm{E}-6$ & $9.3813 \mathrm{E}-7$ \\
\hline 4.190 & 0.045 & 1.782 & 0.210 & 1520 & $4.6279 \mathrm{E}+9$ & $1.3224 \mathrm{E}+9$ & $2.6706 \mathrm{E}-6$ & $6.8189 \mathrm{E}-7$ \\
\hline 7.067 & 2.088 & 3.137 & 0.530 & 1520 & $3.8281 \mathrm{E}+9$ & $1.5980 \mathrm{E}+9$ & $3.1454 \mathrm{E}-6$ & $3.0530 \mathrm{E}-6$ \\
\hline 16.603 & 4.435 & 6.709 & 1.215 & 1520 & $4.2802 \mathrm{E}+9$ & $1.8741 \mathrm{E}+9$ & $1.5512 \mathrm{E}-6$ & $1.5259 \mathrm{E}-6$ \\
\hline 19.503 & 2.328 & 10.933 & 0.986 & 1520 & $1.9625 \mathrm{E}+9$ & $1.5375 \mathrm{E}+8$ & $1.7893 \mathrm{E}-6$ & $3.5168 \mathrm{E}-7$ \\
\hline
\end{tabular}


Table E.2. $\beta$ for high beam intensity hydrogen, calculated at equilibrium

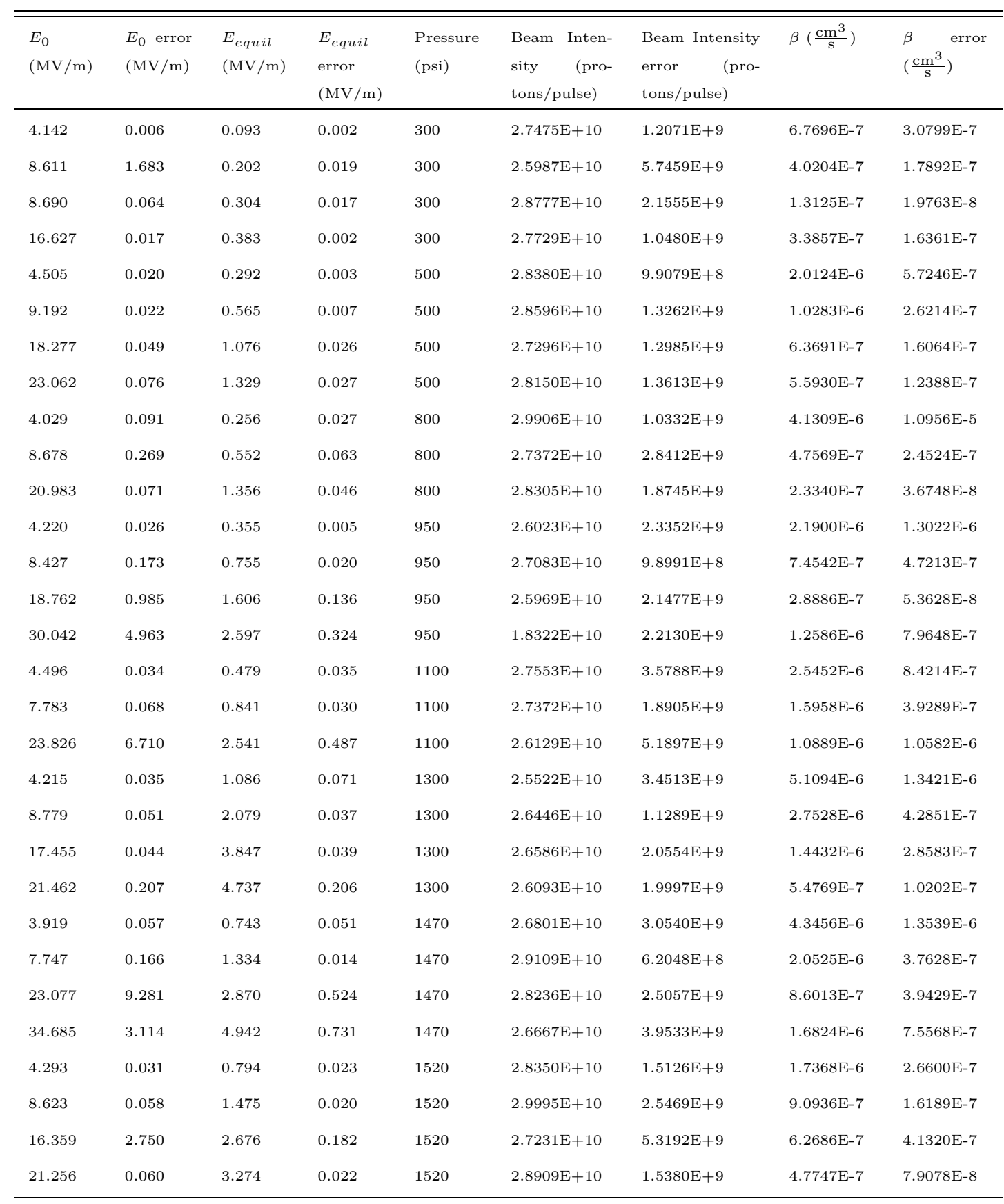


Table E.3. $\beta$ for hydrogen, calculated using the recursion formula. $\beta(E)=\beta_{0} E^{\beta e x p}$

\begin{tabular}{|c|c|c|c|c|}
\hline Pressure (psi) & $\begin{array}{l}\text { Beam Intensity } \\
\text { (protons/pulse) }\end{array}$ & $E_{0}(\mathrm{MV} / \mathrm{m})$ & $\beta_{0}$ & $\beta \exp$ \\
\hline 300 & $2.7475 \mathrm{E}+10$ & 4.143 & $4.0831 \mathrm{E}-8$ & 0.8351 \\
\hline 300 & $2.7882 \mathrm{E}+10$ & 9.177 & $4.0831 \mathrm{E}-8$ & 0.8351 \\
\hline 300 & $2.7729 \mathrm{E}+10$ & 16.632 & $4.0831 \mathrm{E}-8$ & 0.8351 \\
\hline 500 & $2.8380 \mathrm{E}+10$ & 4.509 & $6.0769 \mathrm{E}-7$ & 0.0866 \\
\hline 500 & $2.8596 \mathrm{E}+10$ & 9.203 & $4.0512 \mathrm{E}-7$ & 0.0866 \\
\hline 500 & $2.7296 \mathrm{E}+10$ & 18.288 & $2.7008 \mathrm{E}-7$ & 0.0866 \\
\hline 500 & $2.8150 \mathrm{E}+10$ & 23.073 & $2.0256 \mathrm{E}-7$ & 0.0866 \\
\hline 800 & $2.9748 \mathrm{E}+10$ & 4.119 & $4.8049 \mathrm{E}-7$ & 0.0165 \\
\hline 800 & $2.8216 \mathrm{E}+10$ & 8.382 & $3.4702 \mathrm{E}-7$ & 0.0165 \\
\hline 800 & $2.8305 \mathrm{E}+10$ & 20.982 & $2.1355 \mathrm{E}-7$ & 0.0165 \\
\hline 950 & $2.6023 \mathrm{E}+10$ & 4.219 & $4.7118 \mathrm{E}-7$ & 0.0122 \\
\hline 950 & $2.7083 \mathrm{E}+10$ & 8.424 & $2.9449 \mathrm{E}-7$ & 0.0122 \\
\hline 950 & $2.6337 \mathrm{E}+10$ & 17.848 & $2.3559 \mathrm{E}-7$ & 0.0122 \\
\hline 950 & $2.6459 \mathrm{E}+10$ & 19.761 & $2.0614 \mathrm{E}-7$ & 0.0122 \\
\hline 1100 & $2.8849 \mathrm{E}+10$ & 4.485 & $6.9480 \mathrm{E}-7$ & 0.0061 \\
\hline 1100 & $2.7372 \mathrm{E}+10$ & 7.779 & $4.6320 \mathrm{E}-7$ & 0.0061 \\
\hline 1100 & $2.8029 \mathrm{E}+10$ & 17.444 & $2.7792 \mathrm{E}-7$ & 0.0061 \\
\hline 1100 & $2.8718 \mathrm{E}+10$ & 20.061 & $2.3160 \mathrm{E}-7$ & 0.0061 \\
\hline 1300 & $2.7493 \mathrm{E}+10$ & 4.225 & $1.2132 \mathrm{E}-6$ & 0.0129 \\
\hline 1300 & $2.6734 \mathrm{E}+10$ & 8.775 & $8.4927 \mathrm{E}-7$ & 0.0129 \\
\hline 1300 & $2.6586 \mathrm{E}+10$ & 17.455 & $4.8529 \mathrm{E}-7$ & 0.0129 \\
\hline 1300 & $2.6727 \mathrm{E}+10$ & 21.415 & $3.6397 \mathrm{E}-7$ & 0.0129 \\
\hline 1470 & $2.7842 \mathrm{E}+10$ & 3.912 & $8.9699 \mathrm{E}-7$ & 0.0095 \\
\hline 1470 & $2.9109 \mathrm{E}+10$ & 7.747 & $5.3819 \mathrm{E}-7$ & 0.0095 \\
\hline 1470 & $2.8774 \mathrm{E}+10$ & 15.674 & $3.2889 \mathrm{E}-7$ & 0.0095 \\
\hline 1470 & $2.9274 \mathrm{E}+10$ & 20.636 & $2.6910 \mathrm{E}-7$ & 0.0095 \\
\hline 1520 & $2.8673 \mathrm{E}+10$ & 4.286 & $7.6035 \mathrm{E}-7$ & 0.0092 \\
\hline 1520 & $2.9995 \mathrm{E}+10$ & 8.623 & $4.8386 \mathrm{E}-7$ & 0.0092 \\
\hline 1520 & $2.8817 \mathrm{E}+10$ & 17.222 & $3.1797 \mathrm{E}-7$ & 0.0092 \\
\hline 1520 & $2.8909 \mathrm{E}+10$ & 21.259 & $2.7649 \mathrm{E}-7$ & 0.0092 \\
\hline 300 & $1.5480 \mathrm{E}+09$ & 4.465 & $1.3551 \mathrm{E}-7$ & 0.3885 \\
\hline 300 & $1.6387 \mathrm{E}+09$ & 9.047 & $1.3551 \mathrm{E}-7$ & 0.3885 \\
\hline 300 & $1.6967 \mathrm{E}+09$ & 16.456 & $8.1305 \mathrm{E}-8$ & 0.3885 \\
\hline 500 & $1.4213 \mathrm{E}+09$ & 4.196 & $4.9617 \mathrm{E}-7$ & 0.0827 \\
\hline 500 & $1.4480 \mathrm{E}+09$ & 8.960 & $3.3078 \mathrm{E}-7$ & 0.0827 \\
\hline 500 & $1.4604 \mathrm{E}+09$ & 18.420 & $1.9847 \mathrm{E}-7$ & 0.0827 \\
\hline 500 & $1.4656 \mathrm{E}+09$ & 22.776 & $1.9847 \mathrm{E}-7$ & 0.0827 \\
\hline 800 & $1.3956 \mathrm{E}+09$ & 3.907 & $8.4965 \mathrm{E}-7$ & 0.0426 \\
\hline 800 & $1.5945 \mathrm{E}+09$ & 8.244 & $5.2286 \mathrm{E}-7$ & 0.0426 \\
\hline
\end{tabular}




\begin{tabular}{lllll}
\hline \hline Pressure (psi) & $\begin{array}{l}\text { Beam Intensity } \\
\text { (protons/pulse) }\end{array}$ & $E_{0}(\mathrm{MV} / \mathrm{m})$ & $\beta_{0}$ & $\beta \exp$ \\
\hline 800 & $1.4283 \mathrm{E}+09$ & 21.224 & $2.8757 \mathrm{E}-7$ & 0.0426 \\
950 & $1.6611 \mathrm{E}+09$ & 4.390 & $9.5945 \mathrm{E}-7$ & 0.0437 \\
950 & $1.6825 \mathrm{E}+09$ & 8.966 & $6.7162 \mathrm{E}-7$ & 0.0437 \\
950 & $1.6964 \mathrm{E}+09$ & 18.128 & $4.2216 \mathrm{E}-7$ & 0.0437 \\
950 & $1.6074 \mathrm{E}+09$ & 22.734 & $2.8784 \mathrm{E}-7$ & 0.0437 \\
1100 & $1.7182 \mathrm{E}+09$ & 4.355 & $1.6096 \mathrm{E}-6$ & 0.0391 \\
1100 & $1.5566 \mathrm{E}+09$ & 8.543 & $9.6578 \mathrm{E}-7$ & 0.0391 \\
1100 & $1.7012 \mathrm{E}+09$ & 17.264 & $5.4727 \mathrm{E}-7$ & 0.0391 \\
1100 & $1.6873 \mathrm{E}+09$ & 22.817 & $4.1850 \mathrm{E}-7$ & 0.0391 \\
1300 & $1.9839 \mathrm{E}+09$ & 4.159 & $2.2121 \mathrm{E}-7$ & 0.0350 \\
1300 & $1.9342 \mathrm{E}+09$ & 8.585 & $1.2290 \mathrm{E}-7$ & 0.0350 \\
1300 & $1.9280 \mathrm{E}+09$ & 17.345 & $7.3738 \mathrm{E}-8$ & 0.0350 \\
1300 & $1.9012 \mathrm{E}+09$ & 21.835 & $7.3738 \mathrm{E}-8$ & 0.0350 \\
1520 & $2.1190 \mathrm{E}+09$ & 4.181 & $2.5257 \mathrm{E}-6$ & 0.0247 \\
1520 & $1.8424 \mathrm{E}+09$ & 8.238 & $1.2629 \mathrm{E}-6$ & 0.0247 \\
1520 & $1.8930 \mathrm{E}+09$ & 17.296 & $6.3143 \mathrm{E}-7$ & 0.0247 \\
1520 & $1.9693 \mathrm{E}+09$ & 21.681 & $5.3672 \mathrm{E}-7$ & 0.0247 \\
\hline & & & & \\
\hline & & & & \\
\hline
\end{tabular}


APPENDIX F

ATTACHMENT RATE DATA 
Table F.1. $\tau$ for dry air-doped hydrogen, calculated using the recursion formula.

\begin{tabular}{|c|c|c|c|c|}
\hline Pressure (psi) & $\begin{array}{l}\text { Concentration } \\
(\%)\end{array}$ & $E_{0}$ & $\begin{array}{l}\text { Beam Intensity } \\
\text { (protons/pulse) }\end{array}$ & $\tau(\mathrm{s})$ \\
\hline 300 & 0.04 & 15.50 & $2.7277 \mathrm{E}+10$ & $2.4803 \mathrm{E}-7$ \\
\hline 300 & 0.2 & 12.57 & $2.6719 \mathrm{E}+10$ & $5.8487 \mathrm{E}-8$ \\
\hline 300 & 1 & 16.59 & $2.8201 \mathrm{E}+10$ & $2.7422 \mathrm{E}-8$ \\
\hline 300 & 5 & 16.43 & $2.7069 \mathrm{E}+10$ & $7.1093 \mathrm{E}-9$ \\
\hline 300 & 0.04 & 8.36 & $2.6637 \mathrm{E}+10$ & $1.2704 \mathrm{E}-7$ \\
\hline 300 & 0.2 & 8.75 & $2.7775 \mathrm{E}+10$ & $4.0470 \mathrm{E}-8$ \\
\hline 300 & 1 & 8.93 & $2.7917 \mathrm{E}+10$ & $1.5144 \mathrm{E}-8$ \\
\hline 300 & 5 & 8.91 & $2.6791 \mathrm{E}+10$ & $5.0203 \mathrm{E}-9$ \\
\hline 300 & 0.04 & 4.33 & $2.6949 \mathrm{E}+10$ & $7.4484 \mathrm{E}-8$ \\
\hline 300 & 0.2 & 4.31 & $2.7313 \mathrm{E}+10$ & $2.2600 \mathrm{E}-8$ \\
\hline 300 & 1 & 4.43 & $2.8014 \mathrm{E}+10$ & $7.9314 \mathrm{E}-9$ \\
\hline 300 & 5 & 4.35 & $2.6075 \mathrm{E}+10$ & $2.5029 \mathrm{E}-9$ \\
\hline 800 & 0.04 & 35.03 & $6.3349 \mathrm{E}+09$ & $2.6595 \mathrm{E}-8$ \\
\hline 800 & 1 & 34.29 & $7.1220 \mathrm{E}+09$ & $5.0021 \mathrm{E}-9$ \\
\hline 800 & 0.04 & 21.46 & $6.7448 \mathrm{E}+09$ & $1.4136 \mathrm{E}-8$ \\
\hline 800 & 0.2 & 21.54 & $6.7540 \mathrm{E}+09$ & $1.0387 \mathrm{E}-8$ \\
\hline 800 & 1 & 21.04 & $6.9352 \mathrm{E}+09$ & $6.0629 \mathrm{E}-9$ \\
\hline 800 & 0.2 & 17.34 & $7.0304 \mathrm{E}+09$ & $5.5417 \mathrm{E}-9$ \\
\hline 800 & 0.04 & 8.52 & $6.8944 \mathrm{E}+09$ & $9.1978 \mathrm{E}-9$ \\
\hline 800 & 0.2 & 8.44 & $7.0411 \mathrm{E}+09$ & $8.7738 \mathrm{E}-9$ \\
\hline 800 & 1 & 8.39 & $6.8840 \mathrm{E}+09$ & $2.9793 \mathrm{E}-9$ \\
\hline 1100 & 1 & 22.85 & $2.7870 \mathrm{E}+10$ & $1.5416 \mathrm{E}-9$ \\
\hline 1100 & 5 & 20.98 & $2.7375 \mathrm{E}+10$ & $7.0398 \mathrm{E}-10$ \\
\hline 1100 & 6.78 & 20.98 & $2.6647 \mathrm{E}+10$ & $7.0436 \mathrm{E}-10$ \\
\hline 1100 & 1 & 17.87 & $2.7554 \mathrm{E}+10$ & $1.0222 \mathrm{E}-9$ \\
\hline 1100 & 5 & 17.25 & $2.7218 \mathrm{E}+10$ & $9.5233 \mathrm{E}-10$ \\
\hline 1100 & 6.78 & 15.91 & $2.6750 \mathrm{E}+10$ & $8.0990 \mathrm{E}-10$ \\
\hline 1100 & 1 & 8.95 & $2.7674 \mathrm{E}+10$ & $7.1610 \mathrm{E}-10$ \\
\hline 1100 & 5 & 8.12 & $2.7105 \mathrm{E}+10$ & $6.4924 \mathrm{E}-10$ \\
\hline 1100 & 6.78 & 7.95 & $2.7360 \mathrm{E}+10$ & $6.3588 \mathrm{E}-10$ \\
\hline 1100 & 1 & 4.37 & $2.6983 \mathrm{E}+10$ & $5.2440 \mathrm{E}-10$ \\
\hline 1100 & 5 & 3.96 & $2.7211 \mathrm{E}+10$ & $4.7542 \mathrm{E}-10$ \\
\hline 1100 & 6.78 & 3.84 & $2.6578 \mathrm{E}+10$ & $4.6024 \mathrm{E}-10$ \\
\hline 1470 & 0.001 & 20.26 & $2.9731 \mathrm{E}+10$ & $3.2614 \mathrm{E}-8$ \\
\hline 1470 & 0.002 & 20.42 & $2.8845 \mathrm{E}+10$ & $2.3369 \mathrm{E}-8$ \\
\hline 1470 & 0.01 & 20.82 & $2.7833 \mathrm{E}+10$ & $5.8804 \mathrm{E}-9$ \\
\hline 1470 & 0.025 & 20.45 & $2.8704 \mathrm{E}+10$ & $1.6763 \mathrm{E}-9$ \\
\hline 1470 & 0.05 & 20.49 & $2.6776 \mathrm{E}+10$ & $1.1240 \mathrm{E}-9$ \\
\hline 1470 & 1 & 21.07 & $2.7230 \mathrm{E}+10$ & $9.1331 \mathrm{E}-10$ \\
\hline
\end{tabular}




\begin{tabular}{|c|c|c|c|c|}
\hline Pressure (psi) & $\begin{array}{l}\text { Concentration } \\
(\%)\end{array}$ & $E_{0}$ & $\begin{array}{l}\text { Beam Intensity } \\
\text { (protons/pulse) }\end{array}$ & $\tau(\mathrm{s})$ \\
\hline 1470 & 0.001 & 15.94 & $2.9286 \mathrm{E}+10$ & $2.7128 \mathrm{E}-8$ \\
\hline 1470 & 0.002 & 16.38 & $2.8946 \mathrm{E}+10$ & $1.9074 \mathrm{E}-8$ \\
\hline 1470 & 1 & 16.85 & $2.7691 \mathrm{E}+10$ & $1.2739 \mathrm{E}-9$ \\
\hline 1470 & 0.001 & 7.32 & $2.8477 \mathrm{E}+10$ & $1.7034 \mathrm{E}-8$ \\
\hline 1470 & 0.002 & 7.71 & $2.8441 \mathrm{E}+10$ & $1.1528 \mathrm{E}-8$ \\
\hline 1470 & 1 & 8.16 & $2.7646 \mathrm{E}+10$ & $7.3877 \mathrm{E}-10$ \\
\hline 1470 & 0.001 & 3.86 & $2.8808 \mathrm{E}+10$ & $1.5543 \mathrm{E}-8$ \\
\hline 1470 & 0.002 & 3.96 & $2.8645 \mathrm{E}+10$ & $9.5641 \mathrm{E}-9$ \\
\hline 1470 & 1 & 4.17 & $2.7694 \mathrm{E}+10$ & $7.7832 \mathrm{E}-10$ \\
\hline 300 & 0.04 & 15.59 & $6.6010 \mathrm{E}+09$ & $2.3703 \mathrm{E}-7$ \\
\hline 300 & 0.2 & 16.37 & $6.8533 \mathrm{E}+09$ & $1.5061 \mathrm{E}-7$ \\
\hline 300 & 1 & 16.43 & $6.9621 \mathrm{E}+09$ & $3.2870 \mathrm{E}-8$ \\
\hline 300 & 0.04 & 8.95 & $6.7412 \mathrm{E}+09$ & $1.2537 \mathrm{E}-7$ \\
\hline 300 & 0.2 & 9.07 & $6.8093 \mathrm{E}+09$ & $7.2583 \mathrm{E}-8$ \\
\hline 300 & 1 & 9.00 & $6.9051 \mathrm{E}+09$ & $1.7992 \mathrm{E}-8$ \\
\hline 1100 & 0.04 & 34.14 & $7.1347 \mathrm{E}+09$ & $9.8768 \mathrm{E}-9$ \\
\hline 1100 & 0.2 & 34.55 & $7.5542 \mathrm{E}+09$ & $3.8323 \mathrm{E}-9$ \\
\hline 1100 & 1 & 34.90 & $6.7271 \mathrm{E}+09$ & $1.3159 \mathrm{E}-9$ \\
\hline 1100 & 0.2 & 21.60 & $6.8978 \mathrm{E}+09$ & $2.2913 \mathrm{E}-9$ \\
\hline 1100 & 1 & 22.28 & $6.6771 \mathrm{E}+09$ & $7.1616 \mathrm{E}-10$ \\
\hline 1100 & 0.04 & 8.59 & $8.4003 \mathrm{E}+09$ & $2.2590 \mathrm{E}-9$ \\
\hline 1100 & 0.2 & 8.78 & $6.9091 \mathrm{E}+09$ & $1.2040 \mathrm{E}-9$ \\
\hline 1100 & 1 & 8.85 & $6.7149 \mathrm{E}+09$ & $6.4036 \mathrm{E}-10$ \\
\hline
\end{tabular}


APPENDIX G

ION-ION RECOMBINATION DATA 
Table G.1. $\eta$ for dry air-doped hydrogen, calculated using the recursion formula.

\begin{tabular}{|c|c|c|c|c|}
\hline Pressure (psi) & $\begin{array}{l}\text { Concentration } \\
(\%)\end{array}$ & $E_{0}$ & $\begin{array}{l}\text { Beam Intensity } \\
\text { (protons/pulse) }\end{array}$ & $\eta\left(\frac{\mathrm{cm}^{3}}{\mathrm{~s}}\right)$ \\
\hline 300 & 0.04 & 15.50 & $2.7277 \mathrm{E}+10$ & $1.2401 \mathrm{E}-7$ \\
\hline 300 & 0.2 & 12.57 & $2.6719 \mathrm{E}+10$ & $1.0052 \mathrm{E}-7$ \\
\hline 300 & 1 & 16.59 & $2.8201 \mathrm{E}+10$ & $5.3102 \mathrm{E}-8$ \\
\hline 300 & 5 & 16.43 & $2.7069 \mathrm{E}+10$ & $8.8743 \mathrm{E}-8$ \\
\hline 300 & 0.04 & 8.36 & $2.6637 \mathrm{E}+10$ & $8.0236 \mathrm{E}-8$ \\
\hline 300 & 0.2 & 8.75 & $2.7775 \mathrm{E}+10$ & $5.5996 \mathrm{E}-8$ \\
\hline 300 & 1 & 8.93 & $2.7917 \mathrm{E}+10$ & $7.1421 \mathrm{E}-8$ \\
\hline 300 & 5 & 8.91 & $2.6791 \mathrm{E}+10$ & $1.2119 \mathrm{E}-7$ \\
\hline 300 & 0.04 & 4.33 & $2.6949 \mathrm{E}+10$ & $5.8895 \mathrm{E}-8$ \\
\hline 300 & 0.2 & 4.31 & $2.7313 \mathrm{E}+10$ & $6.5510 \mathrm{E}-8$ \\
\hline 300 & 1 & 4.43 & $2.8014 \mathrm{E}+10$ & $8.5031 \mathrm{E}-8$ \\
\hline 300 & 5 & 4.35 & $2.6075 \mathrm{E}+10$ & $1.3907 \mathrm{E}-7$ \\
\hline 800 & 0.04 & 35.03 & $6.3349 \mathrm{E}+09$ & $4.2034 \mathrm{E}-8$ \\
\hline 800 & 1 & 34.29 & $7.1220 \mathrm{E}+09$ & $3.8410 \mathrm{E}-8$ \\
\hline 800 & 0.04 & 21.46 & $6.7448 \mathrm{E}+09$ & $5.1513 \mathrm{E}-8$ \\
\hline 800 & 0.2 & 21.54 & $6.7540 \mathrm{E}+09$ & $5.1696 \mathrm{E}-8$ \\
\hline 800 & 1 & 21.04 & $6.9352 \mathrm{E}+09$ & $5.8906 \mathrm{E}-8$ \\
\hline 800 & 0.2 & 17.34 & $7.0304 \mathrm{E}+09$ & $5.5502 \mathrm{E}-8$ \\
\hline 800 & 0.04 & 8.52 & $6.8944 \mathrm{E}+09$ & $1.1085 \mathrm{E}-7$ \\
\hline 800 & 0.2 & 8.44 & $7.0411 \mathrm{E}+09$ & $1.1930 \mathrm{E}-7$ \\
\hline 800 & 1 & 8.39 & $6.8840 \mathrm{E}+09$ & $1.1716 \mathrm{E}-7$ \\
\hline 1100 & 1 & 22.85 & $2.7870 \mathrm{E}+10$ & $5.0139 \mathrm{E}-8$ \\
\hline 1100 & 5 & 20.98 & $2.7375 \mathrm{E}+10$ & $9.1517 \mathrm{E}-8$ \\
\hline 1100 & 6.78 & 20.98 & $2.6647 \mathrm{E}+10$ & $8.4575 \mathrm{E}-8$ \\
\hline 1100 & 1 & 17.87 & $2.7554 \mathrm{E}+10$ & $4.8358 \mathrm{E}-8$ \\
\hline 1100 & 5 & 17.25 & $2.7218 \mathrm{E}+10$ & $7.4518 \mathrm{E}-8$ \\
\hline 1100 & 6.78 & 15.91 & $2.6750 \mathrm{E}+10$ & $9.1374 \mathrm{E}-8$ \\
\hline 1100 & 1 & 8.95 & $2.7674 \mathrm{E}+10$ & $6.4101 \mathrm{E}-8$ \\
\hline 1100 & 5 & 8.12 & $2.7105 \mathrm{E}+10$ & $1.0538 \mathrm{E}-7$ \\
\hline 1100 & 6.78 & 7.95 & $2.7360 \mathrm{E}+10$ & $1.2130 \mathrm{E}-7$ \\
\hline 1100 & 1 & 4.37 & $2.6983 \mathrm{E}+10$ & $7.6387 \mathrm{E}-8$ \\
\hline 1100 & 5 & 3.96 & $2.7211 \mathrm{E}+10$ & $1.1301 \mathrm{E}-7$ \\
\hline 1100 & 6.78 & 3.84 & $2.6578 \mathrm{E}+10$ & $1.1768 \mathrm{E}-7$ \\
\hline 1470 & 0.001 & 20.26 & $2.9731 \mathrm{E}+10$ & $1.6208 \mathrm{E}-8$ \\
\hline 1470 & 0.002 & 20.42 & $2.8845 \mathrm{E}+10$ & $2.2058 \mathrm{E}-8$ \\
\hline 1470 & 0.01 & 20.82 & $2.7833 \mathrm{E}+10$ & $3.1642 \mathrm{E}-8$ \\
\hline 1470 & 0.025 & 20.45 & $2.8704 \mathrm{E}+10$ & $3.9271 \mathrm{E}-8$ \\
\hline 1470 & 0.05 & 20.49 & $2.6776 \mathrm{E}+10$ & $4.2629 \mathrm{E}-8$ \\
\hline 1470 & 1 & 21.07 & $2.7230 \mathrm{E}+10$ & $4.6165 \mathrm{E}-8$ \\
\hline
\end{tabular}




\begin{tabular}{|c|c|c|c|c|}
\hline Pressure (psi) & $\begin{array}{l}\text { Concentration } \\
(\%)\end{array}$ & $E_{0}$ & $\begin{array}{l}\text { Beam Intensity } \\
\text { (protons/pulse) }\end{array}$ & $\eta\left(\frac{\mathrm{cm}^{3}}{\mathrm{~s}}\right)$ \\
\hline 1470 & 0.001 & 15.94 & $2.9286 \mathrm{E}+10$ & $2.1045 \mathrm{E}-8$ \\
\hline 1470 & 0.002 & 16.38 & $2.8946 \mathrm{E}+10$ & $2.7519 \mathrm{E}-8$ \\
\hline 1470 & 1 & 16.85 & $2.7691 \mathrm{E}+10$ & $5.9053 \mathrm{E}-8$ \\
\hline 1470 & 0.001 & 7.32 & $2.8477 \mathrm{E}+10$ & $2.9283 \mathrm{E}-8$ \\
\hline 1470 & 0.002 & 7.71 & $2.8441 \mathrm{E}+10$ & $3.0822 \mathrm{E}-8$ \\
\hline 1470 & 1 & 8.16 & $2.7646 \mathrm{E}+10$ & $7.4562 \mathrm{E}-8$ \\
\hline 1470 & 0.001 & 3.86 & $2.8808 \mathrm{E}+10$ & $3.0890 \mathrm{E}-8$ \\
\hline 1470 & 0.002 & 3.96 & $2.8645 \mathrm{E}+10$ & $3.1688 \mathrm{E}-8$ \\
\hline 1470 & 1 & 4.17 & $2.7694 \mathrm{E}+10$ & $8.3554 \mathrm{E}-8$ \\
\hline 300 & 0.04 & 15.59 & $6.6010 \mathrm{E}+09$ & $1.2475 \mathrm{E}-7$ \\
\hline 300 & 0.2 & 16.37 & $6.8533 \mathrm{E}+09$ & $1.3096 \mathrm{E}-7$ \\
\hline 300 & 1 & 16.43 & $6.9621 \mathrm{E}+09$ & $3.9444 \mathrm{E}-8$ \\
\hline 300 & 0.04 & 8.95 & $6.7412 \mathrm{E}+09$ & $1.7910 \mathrm{E}-7$ \\
\hline 300 & 0.2 & 9.07 & $6.8093 \mathrm{E}+09$ & $9.0729 \mathrm{E}-8$ \\
\hline 300 & 1 & 9.00 & $6.9051 \mathrm{E}+09$ & $8.9959 \mathrm{E}-8$ \\
\hline 1100 & 0.04 & 34.14 & $7.1347 \mathrm{E}+09$ & $6.5282 \mathrm{E}-8$ \\
\hline 1100 & 0.2 & 34.55 & $7.5542 \mathrm{E}+09$ & $6.6836 \mathrm{E}-8$ \\
\hline 1100 & 1 & 34.90 & $6.7271 \mathrm{E}+09$ & $6.8218 \mathrm{E}-8$ \\
\hline 1100 & 0.2 & 21.60 & $6.8978 \mathrm{E}+09$ & $7.4664 \mathrm{E}-8$ \\
\hline 1100 & 1 & 22.28 & $6.6771 \mathrm{E}+09$ & $7.9434 \mathrm{E}-8$ \\
\hline 1100 & 0.04 & 8.59 & $8.4003 \mathrm{E}+09$ & $8.2693 \mathrm{E}-8$ \\
\hline 1100 & 0.2 & 8.78 & $6.9091 \mathrm{E}+09$ & $8.6418 \mathrm{E}-8$ \\
\hline 1100 & 1 & 8.85 & $6.7149 \mathrm{E}+09$ & $1.4150 \mathrm{E}-7$ \\
\hline
\end{tabular}




\section{BIBLIOGRAPHY}

[1] M.A. Palmer and W. Chou. International Committee for Future Accelerators Beam Dynamics Newsletter. Number 55. Particle and Fields Commision of IUPAP, August 2011.

[2] G. Aad et al (The ATLAS Collaboration). Observation of a New Particle in the Search for the Standard Model Higgs Boson with the ATLAS Detecter at the LHC. Phys. Lett. B, 716(1):1-29, 2012.

[3] S. Chatrchyan et al (The CMS Collaboration). Observation of a New Boson at a Mass of $125 \mathrm{GeV}$ with the CMS Experiment at the LHC. Phys. Lett. B, 716(1):30-61, 2012.

[4] P. Adamson et al (The MINOS Collaboration). Measurements of Atmospheric Neutrinos and Antineutrinos in the MINOS Far Detector. Phys. Rev. D, 86(5):052007, 2012.

[5] K. Abe et al (The T2K Collaboration). First Muon-Neutrino Disappearance Study with an Off-Axis Beam. Phys. Rev. D, 85(3):031103, 2012.

[6] F.P. An et al (The Daya Bay Collaboration). Improved Measurement of Electron Antineutrino Disappearance at Daya Bay. 2012.

[7] Y. Abe et al (The Double Chooz Collaboration). Indication of Reactor $\bar{\nu}_{e}$ Disappearance in the Double Chooz Experiment. Phys. Rev. Lett., 108(13):131801, 2012.

[8] J.K. Ahn (The RENO Collaboration). Obervation of Reactor Electron Antineutrinos Disapperance in the RENO Experiment. Phys. Rev. Lett., 108(19):191802, 2012.

[9] Mario Conte and W.W. McKay. An Introduction to the Physics of Particle Accelerators. World Scientific Publishing Co. Pte. Ltd., Singapore, 2nd. edition, 2008.

[10] S. Geer. Muon Colliders and Neutrino Factories. Annu. Rev. Nucl. Part. Sci., 59:347-65, 2009.

[11] $R \mathscr{G} D$ Proposal for the National Muon Accelerator Program. FERMILAB-TM2459-APC, Revision 6d, Aug. 2010.

[12] H.G. Kirk et al. The MERIT High-Power Target Experiment at the CERN PS. In Proceedings of EPAC 2008, pages 2886-2888, July 2008.

[13] H.G. Kirk et al. A Solenoid Capture System for a Muon Collider. In Proceedings of PAC 2011, pages 1316-1318, Sept. 2011.

[14] The IDS-NF Collaboration. International Design Study for the Neutrino Factory. Interim design report, Mar. 2011. IDS-NF-020.

[15] Z. Maki, M. Nakagawa, and S. Sakata. Remarks on the Unified Model of Elementary Particles. Prog. Theor. Phys., 28(5), 1962.

[16] J. Beringer et al. (Particle Data Group). PR. D86, 010001, 2012. 
[17] R.H. Bernstein and S.J. Parke. Terrestrial Long-Baseline Neutrino-Oscillation Experiments. Phys. Rev. D, 44(7), 1991.

[18] A.N. Skrinskii and V. Parkhomchuk. Methods of Cooling Beams of Charged Particles. Sov. J. Part. Nucl., 12(3):223, 1981.

[19] D. Neuffer. Principles and Applications of Muon Cooling. Part. Accel., 14:75, 1983.

[20] The MICE Collaboration. An International Muon Ionization Cooling Experiment (MICE). Technical report, Proposal to the Rutherford Appleton Laboratory, 2003.

[21] P. Snopok, G. Hanson, and R. Palmer. Simulations of the Tapered Guggenheim 6D Cooling Channel for the Muon Collider. In Proceeding of PAC 2011, pages 217-219, Sept. 2011.

[22] K. Yonehara et al. A Helical Cooling Channel System for Muon Colliders. In Proceeding of IPAC 2010, pages 870-872, May 2010.

[23] Y. Alexahin. Circularly Inclined Solenoid Channel for 6D Ionization Cooling of Muons. In Proceeding of PAC 2009, pages 727-729, May 2009.

[24] J. Norem et al. Dark Current, Breakdown, and Magnetic Field Effects in a Multicell, $805 \mathrm{MHz}$ Cavity. Phys. Rev. ST Accel. Beams, 6(7), 2003.

[25] D. Stratakis, J.C. Gallardo, and R.B. Palmer. Effect of External Magnetic Fields on the Operation of High-Gradient Accelerating Structures. Nucl. Inst. Meth. Phys. Res. A, 620(2-3), 2010.

[26] A. Moretti et al. Effects of High Solenoidal Magnetic Fields on RF Accelerating Cavities. Phys. Rev. ST Accel. Beams, 8(7), 2005.

[27] A. Moretti et al. Effect of High Solenoidal Magnetic Fields on Breakdown Voltages of High Vacuum $805 \mathrm{MHz}$ Cavities. In Proceedings of LINAC 2004, pages 271-274, Aug. 2004.

[28] D. Huang et al. RF Studies at Fermilab MuCool Test Area. In Proceedings of PAC 2009, pages 888-890, May 2009.

[29] R.P. Johnson et al. Gaseous Hydrogen for Muon Beam Cooling. In Proceedings of PAC 2003, pages 1792-1794, May 2003.

[30] D.J. DeBitetto and L.H. Fisher. Townsend Ionization Coefficients and Uniform Field Breakdown in Hydrogen and Nitrogen at High Pressures. Phys. Rev., 104(5), 1956.

[31] F. Paschen. Wied. Ann., 37:69, 1889.

[32] P. Hanlet et al. High Pressure RF Cavities in Magnetic Fields. In Proceedings of EPAC 2006, pages 1364-1366, June 2006.

[33] K. Yonehara et al. Doped $\mathrm{H}_{2}$-Filled RF Cavities for Muon Beam Cooling. In Proceedings of PAC 2009, pages 855-857, May 2009.

[34] P. Wilson. Theory of RF Breakdown in Accelerating Structures. US Workshop on High Gradient Research for Multi TeV Linear Colliders, July 2005. SLAC. 
[35] I. Ben-Itzhak et al. Ionization and Excitation of Hydrogen Molecules by Fast Proton Impact. J. Phys. B: At. Mol. Opt. Phys, 29:L21, 1996.

[36] J.S. Yoon et al. Cross Sections for Electron Collisions with Hydrogen Molecules. J. Phys. Chem. Ref. Data, 37:913, 2008.

[37] A.V. Phelps. Cross Sections and Swarm Coefficients for $\mathrm{H}^{+}, \mathrm{H}_{2}^{+}, \mathrm{H}_{3}^{+}, \mathrm{H}, \mathrm{H}_{2}$, and $\mathrm{H}^{-}$in $\mathrm{H}_{2}$ for Energies from $0.1 \mathrm{eV}$ to $10 \mathrm{keV}$. J. Phys. Chem. Ref. Data, 19:653, 1990.

[38] J.J. Leventhal and L. Friedman. Energy Transfer in the De-excitation of $\left(\mathrm{H}_{3}^{+}\right)$ by $H_{2}$. J. Chem. Phys, 50:2928, 1968.

[39] N.G. Adams, D. Smith, and E. Alge. Measurements of Dissociative Recombination Coefficients of $\mathrm{H}_{3}^{+}, \mathrm{HCO}^{+}, \mathrm{N}_{2} \mathrm{H}^{+}$, and $\mathrm{CH}_{5}^{+}$at 95 and $300 \mathrm{~K}$ Using the FALP Apparatus. J. Chem. Phys., 81:1778, 1984.

[40] W. Paul, B. Lücke, S. Schlemmer, and D. Gerlich. On the Dynamics of the Reaction of Positive Hydrogen Cluster Ions $\left(\mathrm{H}_{5}^{+}\right.$to $\left.\mathrm{H}_{23}^{+}\right)$with Para and Normal Hydrogen at 10 K. Int. J. Mass Spec. Ion Proc., 149-150:373-387, 1995.

[41] K. Nakamura et al. (Particle Data Group). The Review of Particle Physics. J. Phys. G, 37:075021, 2010.

[42] Y.P. Raizer. Gas Discharge Physics. Springer-Verlag, Berlin, 1991.

[43] Y. Itakawa. Effective Collision Frequency of Electrons in Atmospheric Gases. Planet. Space Sci., 19:993-1007, 1971.

[44] K.Y. Ng. Physics of Intensity Dependent Beam Instabilities. World Scientific Publishing Co. Pte. Ltd., Singapore, 1st. edition, 2006.

[45] A. Chao and M. Tigner. Handbook of Accelerator Physics and Engineering. World Scientific Publishing Co. Pte. Ltd., Singapore, 2nd. edition, 2002.

[46] J.L. Pack and A.V. Phelps. Drift Velocities of Slow Electrons in Helium, Neon, Argon, Hydrogen, and Nitrogen. Phys. Rev., 121:798-806, 1961.

[47] J.J. Lowke. The Drift Velocity of Electrons in Hydrogen and Nitrogen. Aust. J. Phys., 16:115-135, 1962.

[48] A. Bartels. Pressure Dependence of Electron Drift Velocity in Hydrogen at 77.8 K. Phys. Rev. Lett., 28:213-215, 1972.

[49] V.M. Atrazhev and I.T. Iakubov. The Electron Drift Velocity in Dense Gases. J. Phys. D: Appl. Phys., 10:2155, 1977.

[50] S.R. Hunter. Monte Carlo Simulation of Electron Swarms in H2. Aust. J. Phys., 30:83-104, 1977.

[51] G.L. Braglia and V. Dallacasa. Theory of the Density Dependence of Electron Drift Velocity in Gases. Phys. Rev. A, 18:711-717, 1978.

[52] T.F. O’Malley. General Model for Electron Drift and Diffusion in a Dense Gas. J. Phys. B: At. Mol. Opt. Phys., 25:163, 1992. 
[53] A. Bagheri, K.L. Baluja, and S.M. Datta. Density Dependence of Electron Mobility in Dense Gases. Z. Phys. D., 32:211-217, 1994.

[54] A. Bartels. Density Dependence of Electron Drift Velocities in Helium and Hydrogen at 77.6 K. Appl. Phys., 8:59-64, 1975.

[55] L. Frommhold. Resonance Scattering and Drift Motion of Electrons through Gases. Phys. Rev., 172:118-125, 1968.

[56] G.L. Braglia and V. Dallacasa. Theory of Electron Mobility in Dense Gases. Phys. Rev. A, 26(2), 1982.

[57] T.F. O'Malley. Electron Distribution and Mobility in a Dense Gas of Repulsive Scatterers. J. Phys. B: At. Mol. Opt. Phys., 22:3701-3708, 1989.

[58] T.F. O'Malley. Multiple Scattering Effect on Electron Mobilities in Dense Gases. J. Phys. B: At. Mol. Phys., 13:1491-1504, 1980.

[59] H.R. Harrison and B.E. Springett. Electron Mobility Variation in Dense Hydrogen Gas. Chem. Phys. Lett., 10(4):418, 1971.

[60] D.L. Albrittion et al. Mobilities of Mass-Identified $\mathrm{H}_{3}^{+}$and $H^{+}$Ions in Hydrogen. Phys. Rev., 171(1):94, 1968.

[61] H.W. Ellis et al. Transport Properties of Gaseous Ions over a Wide Energy Range. Atom. Data and Nucl. Data Tab., 17:177-210, 1976.

[62] E.A. Mason and J.T. Vanderslice. Mobility of Hydrogen Ions $\left(H^{+}, H_{2}^{+}, \mathrm{H}_{3}^{+}\right)$in Hydrogen. Phys. Rev., 114(2):497, 1959.

[63] T.M. Miller et al. Reaction of $\mathrm{H}^{+}$in $\mathrm{H}_{2}$ and $\mathrm{D}^{+}$in $\mathrm{D}_{2}$; Mobilities of Hydrogen and Alkali Ions in $\mathrm{H}_{2}$ and $\mathrm{D}_{2}$ Gases. Phys. Rev., 173(1):115, 1968.

[64] L.A. Viehland and E.A. Mason. Transport Properties of Gaseous Ions over a Wide Energy Range, IV. Atom. Data and Nucl. Data Tab., 60:37-95, 1995.

[65] M.T. Elford and H.B. Milloy. The Mobility of $\mathrm{H}_{3}^{+}$and $\mathrm{H}_{5}^{+}$Ions in Hydrogen and the Equilibrium Constant for the Reaction $\mathrm{H}_{3}^{+}+2 \mathrm{H}_{2} \leftrightharpoons \mathrm{H}_{5}^{+}+\mathrm{H}_{2}$ at Gas Temperatures of 195, 273 and 293K. Aust. J. Phys., 27:795-811, 1974.

[66] B.M. Smirnov. Diffusion and Mobility of Ions in a Gas. Sov. Phys. Usp., 10(3):313, 1967.

[67] E.W. McDaniel and H.R. Crane. Measurements of the Mobilities of the Negative Ions in Oxygen and in Mixtures of Oxygen with the Noble Gases, Hydrogen, Nitrogen, and Carbon Dioxide. Rev. Sci. Instr., 28(9):684, 1957.

[68] A.G. Engelhardt and A.V. Phelps. Elastic and Inelastic Collision Cross Sections in Hydrogen and Deuterium. Phys. Rev., 131(5):2115, 1963.

[69] L.S. Frost and A.V. Phelps. Rotational Excitement and Momentum Transfer Cross Sections for Electrons in $\mathrm{H}_{2}$ and $\mathrm{N}_{2}$ from Transport Coefficients. Phys. Rev., 127(5):1621, 1962.

[70] A.E.D. Heylen. Calculated Electron Mobility in Hydrogen. Proc. Phys. Soc., $76: 779,1960$. 
[71] R.W. Crompton, M.T. Elford, and A.I. McIntosh. Electron Transport Coefficients in Hydrogen and Deuterium. Aust. J. Phys., 21:43-63, 1968.

[72] R. Johnsen. A Critical Review of $\mathrm{H}_{3}^{+}$Recombination Studies. J. Phys.: Conf. Ser., 4:83, 2005.

[73] R. Johnsen and S.L. Guberman. Dissociative recombination of $\mathrm{H}_{3}^{+}$ion with electrons: Theory and experiment. 2010.

[74] Glosík et al. The Recombination of $\mathrm{H}_{3}^{+}$and $\mathrm{H}_{5}^{+}$ions with Electrons in Hydrogen Plasma: Dependence on Temperature and on Pressure of $\mathrm{H}_{2}$. Plasma Sources Sci. Technol., 12:S117-S122, 2003.

[75] M.T. Leu, M.A. Biondi, and R. Johnsen. Measurements of Recombination of Electrons with $\mathrm{H}_{3}^{+}$and $\mathrm{H}_{5}^{+}$Ions. Phys. Rev. A, 8(1):413, 1973.

[76] J.A. Macdonald, M.A. Biondi, and R. Johnsen. Recombination of Electrons with $\mathrm{H}_{3}^{+}$and $\mathrm{H}_{5}^{+}$Ions. Planet. Space Sci., 32(5):651, 1984.

[77] F. Bloch and N.E. Bradbury. On the Mechanism of Unimolecular Electron Capture. Phys. Rev., 48(8):689, 1935.

[78] N.L. Aleksandrov. Three-Body Electron Attachment to a Molecule. Usp. Fiz. Nauk., 164:177-206, 1988.

[79] J.I. Steinfeld. Molecules and Radiation: An Introduction to Modern Molecular Spectroscopy. The MIT Press, Cambridge, MA, 2nd edition, 1985.

[80] A. Fridman and L.A. Kennedy. Plasma Physics and Engineering. Taylor \& Francis Books, Inc., New York, 1st edition, 2004.

[81] L.M. Chanin, A.V. Phelps, and M.A. Biondi. Measurement of the Attachment of Low-Energy Electrons to Oxygen Molecules. Phys. Rev., 128(1):219, 1962.

[82] I.A. Kossyi. Kinetic Scheme of the Non-Equilibrium Discharge in NitrogenOxygen Mixtures. Plasma Sources Sci. Technol., 1:207-220, 1992.

[83] A. Herzenberg. Attachement of Slow Electrons to Oxygen Molecules. J. Chem. Phys., 51(11):4942, 1969.

[84] A.V. Phelps.

[85] M.N. Hirsh, P.N. Eisner, and J.A. Slevin. Ionization and Attachment in $\mathrm{O}_{2}$ and Airlike $\mathrm{N}_{2}: \mathrm{O}_{2}$ Mixtures Irradiated by $1.5-\mathrm{MeV}$ Electrons. Phys. Rev., 178(1):175, 1969.

[86] D.L. McCorkle, L.G. Christophorou, and V.E. Anderson. Low Energy $(\leq 1 \mathrm{eV})$ Electron Attachment to Molecules at Very High Gas Densities: $O_{2}$. J. Phys. B: Atom. Molec. Phys., 5:1211, 1972.

[87] D. Spense and G.J. Schulz. Three-Body Attachment in $\mathrm{O}_{2}$ Using Electron Beams. Phys. Rev. A, 5(2):724, 1972.

[88] J.L. Pack and A.V. Phelps. Electron Attachment and Detachment. I. Pure $\mathrm{O}_{2}$ at Low Energy. J. Chem. Phys., 44(5):1870, 1966. 
[89] H. Shimamori and Y. Hatano. Thermal Electron Attachment to $\mathrm{O}_{2}$ in $\mathrm{H}_{2}$ and $D_{2}$. Chem. Phys. Lett., 38(2):242, 1976.

[90] A. Fridman. Plasma Chemistry. Cambridge University Press, Cambridge, 1st edition, 2008.

[91] D.R. Bates and M.R. Flannery. Three-Body Ionic Recombination at Moderate and High Gas Densities. J. Phys. B. Atom. Molec. Phys., 2(2):184, 1969.

[92] K.A. Brueckner. Ion-Ion Recombination. J. Chem. Phys., 40(2):439, 1964.

[93] F.T. Chan. Electron-Ion and Ion-Ion Dissociative Recombination of Oxygen. II. Ion-Ion Recombination. J. Chem. Phys., 49(6):2540, 1968.

[94] M.R. Flannery. Theoretical and Exerimental Three-Body Ionic Recombination Coefficients. Phys. Rev. Lett., 21(26):1729, 1968.

[95] M.R. Flannery. Ion-Ion Recombination as a Function of Ion and Gas Densities. Chem. Phys. Lett., 80(3):541, 1981.

[96] I.M. Littlewood and R.E. Pyle. Ion-Ion Recombination in a Gas Discharge. Phys. Rev. A, 41(2):1071, 1990.

[97] W.L. Morgan et al. Theory of Ion-Ion Recombination in Plasmas. Phys. Rev. A, 26(3):1696, 1982.

[98] R.E. Olson. Absorbing-Sphere Model for Calculating Ion-Ion Recombination Total Cross Sections. J. Chem. Phys., 56(6):2979, 1972.

[99] R.E. Olson, J.R. Peterson, and J. Moseley. Ion-Ion Recombination Total Cross Sections-Atomic Species. J. Chem. Phys., 53(9):3391, 1970.

[100] J.D. Jackson. Classical Electrodynamics. John Wiley and Sons, Inc., New York, 3rd edition, 1999.

[101] M. Popovic. The Muon Test Area at Fermilab. In Proceedings of EPAC 2004, pages 812-814, September 2004.

[102] D. Bowring. $805 \mathrm{MHz}$ Button Cavity Results and Replacement Plans. NuFact '12, July 2012.

[103] G. Kazakevich et al. Multi-Purpose $805 \mathrm{MHz}$ Pillbox RF Cavity for Muon Accelerator Studies. In Proceedings of PAC 2011, pages 1003-1005, Sept. 2011.

[104] E.S. McCrory et al. Longitudinal Emittance from the Fermilab 400-MeV Linac. Conf. Proc., C9608262:332-334, 1996.

[105] Fermilab Linac Upgrade Conceptual Design Revision 4A. FERMILAB-LUConceptual Design, 1989.

[106] M.R. Jana et al. Beam Profile Measurement in MTA Beam Line for High Pressure RF Cavity Beam Test. In Proceedings of IPAC 2012, pages 948-950, July 2012.

[107] M.R. Jana et al. Measurement of Transmission Efficiency for $400 \mathrm{MeV}$ Proton Beam through Collimator at Fermilab MuCool Test Area Using Chromox-6 Scintillation Screen. Rev. Sci. Instr., Submitted for review, 2013. 
[108] Fair-Rite Products Corp. http://www.fair-rite.com/newfair/index.htm, March 2013.

[109] Pearson Electronics. http://www. pearsonelectronics.com, March 2013.

[110] Fermi Research Alliance. http://www-ad.fnal.gov/controls/public/ linux-console.html, March 2013.

[111] K. Yonehara. Simulation of Gas Plasma Distribution in High Pressure Gas Filled RF Test Cell. Version 6, Sept. 2012.

[112] D.M. Pozar. Microwave Engineering. John Wiley and Sons, Inc., New York, 3rd edition, 2005.

[113] Q. Kerns, F. Kirsten, and C. Winningstad. Pulse Response of Coaxial Cables. Counting note, Lawrence Radiation Laboratory, Feb. 1964. LBL-CC-2-1A.

[114] C.H. Holbrow et al. Modern Introductory Physics. Springer Science+Business Media, LLC, New York, 2nd edition, 2010.

[115] V. Golovin and V. Saveliev. Novel Type of Avalanche Photodetector with Geiger Mode Operation. Nucl. Inst. Meth. Phys. Res. A, 518:560-564, 2004.

[116] K. Yonehara. MTA High Pressure Cavity April-June 2012 MTA Running. Fermilab Safety Document, 2012.

[117] A. Kurup. An Automated Conditioning System for the MUCOOL Experiments at Fermilab. In Proceedings of IPAC 2011, pages 844-846, September 2011.

[118] Wolfram. Wolfram Mathematica. http://www.wolfram.com/mathematica/, 2013. Version 8.

[119] K. Yonehara. Correction Factor Analysis. Version 3, Nov. 2012.

[120] T. Roberts. G4beamline. http://www .muonsinc.com/muons3/G4beamline, 2013. Muons, Inc.

[121] R. Grünberg. Z. Physik, 204(2), 1967. 